\title{
Dynamics and interactions of the voltage-dependent anion channel 1 studied by NMR spectroscopy
}

\author{
Dissertation \\ zur Erlangung des mathematisch-naturwissenschaftlichen Doktorgrades \\ „Doctor rerum naturalium“ \\ der Georg-August-Universität Göttingen
}

vorgelegt von

Saskia Villinger

aus Paderborn

Göttingen 2012 
Mitglied des Betreuungsauschusses (Referent): Prof. Dr. Christian Griesinger NMR-basierte Strukturbiologie, Max-Planck-Institut für Biophysikalische Chemie

Mitglied des Betreuungsausschusses (Referentin): Prof. Dr. Claudia Steinem Organische und Biomolekulare Chemie, Georg-August-Universität Göttingen

Mitglied des Betreuungsausschusses: Prof. Dr. Peter Rehling Biochemie II, Georg-August-Universität Göttingen

Mitglied des Betreuungsausschusses: Prof. Dr. Markus Zweckstetter NMR-basierte Strukturbiologie, Max-Planck-Institut für Biophysikalische Chemie

Tag der mündlichen Prüfung: 21. Februar 2012 


\section{Affidavit}

I hereby declare that this thesis has been written independently and with no other sources and aids than quoted.

Saskia Villinger 


\begin{abstract}
The voltage-dependent anion channel (VDAC), the most abundant protein in the outer mitochondrial membrane, acts as a gatekeeper for the entry and exit of mitochondrial metabolites and is involved in mitochondrial apoptosis. Recent determination of its high-resolution structure by three independent groups revealed a 19-stranded $\beta$-barrel with a differently arranged $\mathrm{N}$-terminal $\alpha$-helix inside the pore.

In this thesis, the NMR resonance assignment of isoform one of human VDAC (VDAC1) in solution is increased. In addition, this study provides evidence for a kinked $\alpha$-helical structure of the N-terminus, which is compatible with the crystal structure of VDAC1, although other structures cannot be excluded. Furthermore, this study reveals functional dynamics of VDAC1 by a combination of solution NMR spectroscopy, Gaussian network model (GNM) analysis, and molecular dynamics (MD) simulations. Low signal intensities indicate conformational exchange in the second part of the $\mathrm{N}$ terminal $\alpha$-helix and the linker connecting the $\alpha$-helix to the first $\beta$-strand. In addition, mutation of arginine 15 in the second $\alpha$-helical part affects the stability of the $\alpha$-helix and the overall barrel in a complex manner. Micro- to millisecond dynamics are significantly increased in the N-terminal $\alpha$-helix, the linker, and the N-terminal six $\beta$ strands of VDAC1 in micellar solution. In addition, hydrogen bonds are instable in the $\mathrm{N}$-terminal three $\beta$-strands. In agreement, the $\mathrm{N}$-terminal $\beta$-strands exhibit increased $\mathrm{B}$ factors in the crystal structure of VDAC1 and intrinsic instability predicted by the GNM analysis. Mutation or chemical modification of the membrane facing glutamic acid 73 (E73) strongly reduces the micro- to millisecond dynamics in solution. MD simulations reveal that a charge on E73 accounts for the elevation of N-terminal protein dynamics as well as a thinning of the nearby membrane. Since E73 is necessary for hexokinase-Iinduced VDAC1 channel closure and inhibition of apoptosis, these results imply that micro- to millisecond dynamics in the N-terminal part of the $\beta$-barrel are essential for VDAC1 interaction and gating. Moreover, the data suggest that dynamics in the $\alpha$-helix are connected with these processes.
\end{abstract}


Furthermore, this study reveals two binding sites for the pore's most important transport substrate ATP. The location of the ATP binding sites, one of them comprising the $N$-terminal $\alpha$-helix, the linker, and nearby $\beta$-strands, suggests controlled metabolite flux and ligand-induced stabilization of the open state of the VDAC1 pore.

Finally, $\mathrm{Ca}^{2+}$ is found to interact with two distinct $\mathrm{N}$-terminal and C-terminal regions in the $\beta$-barrel. These regions overlap with VDAC1 oligomerization sites and dynamic regions, suggesting a connection between $\mathrm{Ca}^{2+}$ interaction, gating, and oligomerization. 


\section{Zusammenfassung}

Der spannungsabhängige Anionenkanal (engl. "voltage-dependent anion channel", VDAC), das häufigste Protein in der äußeren Mitochondrienmembran, dient als wichtiger Kontrollpunkt für den Ein- und Austritt von mitochondrialen Metaboliten und ist in mitochondriale Apoptose involviert. Die kürzliche Bestimmung der hochaufgelösten Struktur durch drei unabhängige Gruppen weist ein 19-strängiges $\beta$ Fass mit einer unterschiedlich arrangierten N-terminalen $\alpha$-Helix in dessen Pore auf.

In dieser Arbeit wird die Resonanzzuordnung der Isoform eins des humanen VDAC (VDAC1) in Lösung erweitert. Des Weiteren werden Nachweise für eine geknickte $\alpha$-helikale Struktur des N-Terminus erbracht, die mit der Kristallstruktur von VDAC1 kompatibel ist, obwohl andere Strukturen nicht ausgeschlossen werden können. Zudem zeigt diese Studie funktionelle Dynamik in VDAC1 mithilfe einer Kombination aus Lösungs-NMR-Spektroskopie, Analyse Gauss'scher Netzwerkmodelle (GNM) und Molekulardynamik-(MD)-Simulationen. Niedrige Signalintensitäten deuten auf das Vorhandensein von Konformationsaustausch im zweiten Teil der N-terminalen $\alpha$-Helix und dem Linker hin, der die $\alpha$-Helix mit dem ersten $\beta$-Strang verbindet. Zusätzlich beeinflusst die Mutation von Arginin 15 im zweiten $\alpha$-helikalen Teil die Stabilität der $\alpha$-Helix und des gesamten $\beta$-Fasses in komplexer Weise. In Mizellen ist die Mikro- bis Millisekunden-Dynamik in der N-terminalen $\alpha$-Helix, dem Linker und den Nterminalen sechs $\beta$-Strängen von VDAC1 signifikant erhöht. Außerdem sind die Wasserstoffbrücken der N-terminalen drei $\beta$-Stränge instabil. Übereinstimmend zeigen die N-terminalen Stränge erhöhte B-Faktoren in der Kristallstruktur von VDAC1 und durch GNM-Analyse vorhergesagte intrinsische Instabilität. Mutation oder chemische Modifizierung der in die Membran weisenden Glutaminsäure E73 reduzieren die in Lösung auftretende Mikro- bis Millisekunden-Dynamik nachhaltig. MD-Simulationen zeigen, dass eine Ladung an der Seitenkette von E73 für die Erhöhung der N-terminalen Proteindynamik sowie für eine Reduktion der Membrandicke in der Umgebung von E73 verantwortlich ist. Da E73 für die durch Hexokinase induzierte Kanalschließung und Inhibierung von Apoptose notwendig ist, zeigen diese Ergebnisse, dass die Mikro- bis 
Millisekunden-Dynamik im N-terminalen $\beta$-Fassbereich essentiell für Interaktionen und Kanalschließung von VDAC1 ist. Außerdem weisen die Daten auf einen Zusammenhang der Helix-Dynamik mit diesen Prozessen hin.

Weiterhin konnten in der vorliegenden Studie zwei Bindungsstellen für das wichtigste Transportsubstrat, ATP, ermittelt werden. Eine dieser Bindungsstellen umfasst die N-terminale $\alpha$-Helix, den Linker und die nahegelegenen $\beta$-Stränge. Die Lokalisierung der ATP-Bindestellen deutet kontrollierten Metabolitenfluss und durch den Liganden induzierte Stabilisierung des offenen Zustands der VDAC1-Pore an.

Zum Abschluss zeigt diese Studie, dass $\mathrm{Ca}^{2+}$ mit zwei unterschiedlichen N- und C-terminalen Bereichen des $\beta$-Fasses interagiert. Diese Regionen überlappen mit Oligomerisierungsstellen und dynamischen Regionen von VDAC1 und weisen daher auf eine Verbindung zwischen $\mathrm{Ca}^{2+}$-Wechselwirkung, Kanalschließung und Oligomerisierung hin. 


\section{Acknowledgements}

This thesis was performed in the department of NMR-based Structural Biology, Max Planck Institute for Biophysical Chemistry, Göttingen, under supervision of Prof. Dr. Christian Griesinger and Prof. Dr. Markus Zweckstetter.

I am deeply grateful to the director of the department, Prof. Dr. Christian Griesinger, for the opportunity to perform my thesis in his department, for providing excellent research facilities, teaching, and stimulating scientific discussions during my thesis.

I would like to express my sincere gratitude to my direct supervisor Prof. Dr. Markus Zweckstetter for the project design and the continuous support, advice, encouragement and stimulating scientific input throughout my thesis.

I owe my gratitude to Prof. Dr. Claudia Steinem and Prof. Dr. Peter Rehling from the Georg August University Göttingen for the membership in my thesis committee and for useful discussions and suggestions during and beyond thesis committee meetings.

I deeply thank Dr. Stefan Becker and Karin Giller for their immense effort in the design and preparation of protein samples, for the helpful discussions about related questions, and for reading the respective sections in this manuscript.

I am sincerely grateful to Dr. Monika Bayrhuber for providing helpful support with NMR spectroscopy, data analysis, and useful insights in the early stage of the project.

I would like to thank Prof. Dr. Bert L. de Groot, Dr. Rodolfo Briones, and Dr. Ulrich Zachariae for MD simulations and a very productive and convenient cooperation.

I thank Dr. Robert Schneider and Dr. Adam Lange for solid state chemical shift information and the efficient cooperation.

I owe gratitude to Prof. Dr. Roland Benz from the Rudolf Virchow Center, DFGResearch Center for Experimental Biomedicine, University of Würzburg, for providing the facilities for bilayer measurements. I am also deeply grateful to Elke Maier, who introduced me into the BLM method with ample patience. 
I would like to thank Sheng Qi Xiang, Dr. Stefan Bibow, Dr. Min-Kyo Cho, Dr. Lukasz Skora, and Dr. Ulrich Dürr for practical help with NMR spectrometers.

I thank Lukasz and Mariusz Jaremko for the demonstration of charged gel preparations for protein alignment.

I thank Dr. Dirk Bockelmann and Heinz-Jürgen Arwe for a very stable system administration and trouble-shooting with programs. I thank Sigrid Silberer and Petra Breiner for organisatorial and administrative help.

I would like to thank Dr. Thomas Meins, Dr. Kornelius Zeth, and Dr. Clemens Vonrhein for help in the initial stage of the project.

I am deeply thankful to Venita Daebel and Dr. Stefan Bibow for the time and effort they spent reading this manuscript and for their exceeding personal support and friendship. I also thank Venita, Stefan, and Fabian for the regular and enjoyable Friday lunch meetings.

I am thankful to all current and former members of this department for the friendly and supporting atmosphere that delighted the every day work: Venita, Stefan, Manuel, Florian, Philipp, Sebastian T., Fabian, Han, Edward, Rakhi, Sheng Qi, Guowei, Hari, Korvin, Benjamin, David, Davood, Eibe, Adam M., Azitar, Min-Kyu, Hai-Young, Laura, Jörg, Steffen, Edith, Ulrich, Lukasz S., Francesca, Luigi, Hessam, Ivonne, Sebastian, Jean-Philippe, Hannes, Antoine, and all other people from NMR II.

I thank the "Fond der Chemischen Industrie" for providing personal and scientific financial support in form of the Kékule stipend. Furthermore, I would like to acknowledge the Max Planck Society and the DFG (Sonderforschungsbereich 803) for financial support.

I thank Nina Sinke and Andrea Schuch for their continuous encouragement during the thesis and for the friendship that survived since the early childhood.

Finally, I would like to thank my family for supporting and encouring me all these years during and prior to my thesis. 


\section{Publication list}

This thesis is based on the following publications:

- Bayrhuber, M., T. Meins, M. Habeck, S. Becker, K. Giller, S. Villinger, C. Vonrhein, C. Griesinger, M. Zweckstetter, and K. Zeth. (2008). Structure of the human voltage-dependent anion channel. Proc Natl Acad Sci U SA. 105:15370-5.

- Villinger, S., R. Briones, K. Giller, U. Zachariae, A. Lange, B.L. de Groot, C. Griesinger, S. Becker, and M. Zweckstetter. (2010). Functional dynamics in the voltage-dependent anion channel. Proc Natl Acad Sci U S A. 107:22546-51. 


\section{Table of contents}

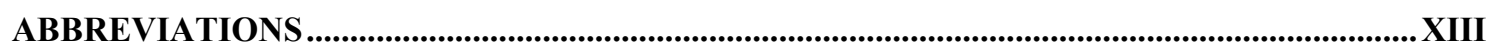

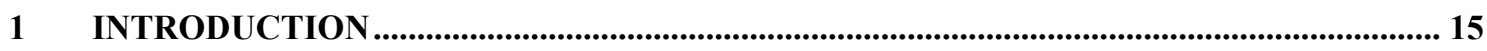

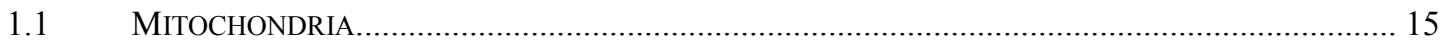

1.1.1 Mitochondria and energy metabolism ....................................................................... 15

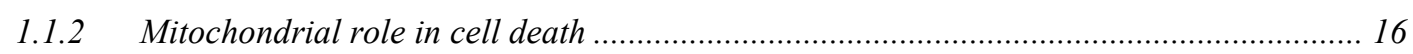

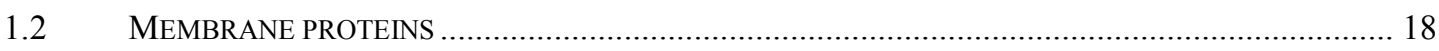

1.2.1 Membrane protein structures and interactions.......................................................... 18

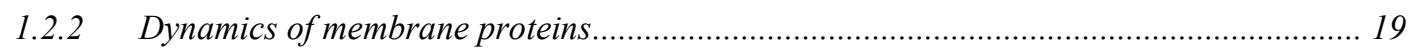

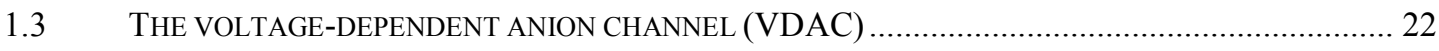

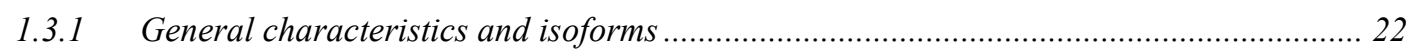

1.3.2 Conductance, selectivity, and gating of VDAC ....................................................... 22

1.3.3 VDAC's role in metabolism and apoptosis ............................................................... 23

1.3.4 The structure of VDAC1 .............................................................................................. 25

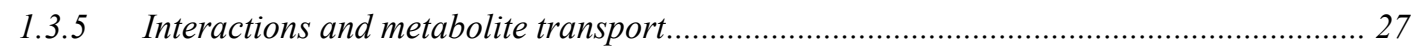

1.3.6 Models for VDAC channel closure .............................................................................. 28

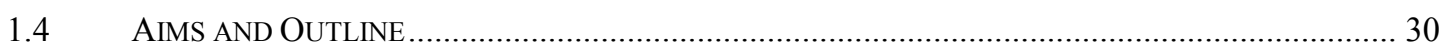

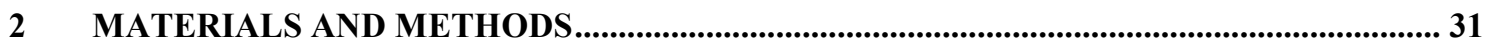

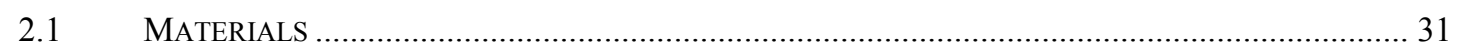

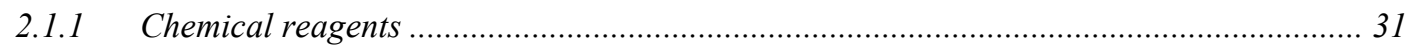

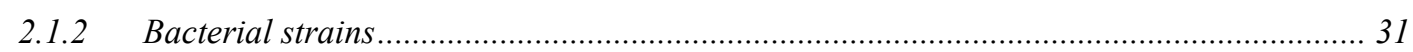

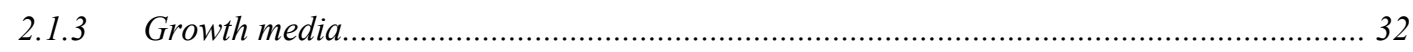

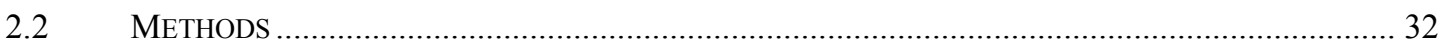

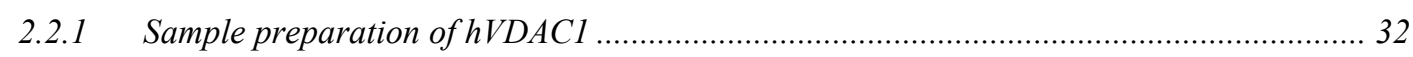

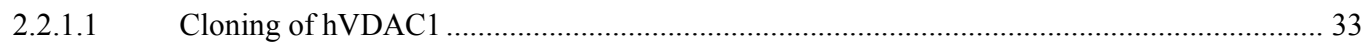

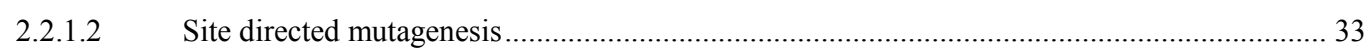

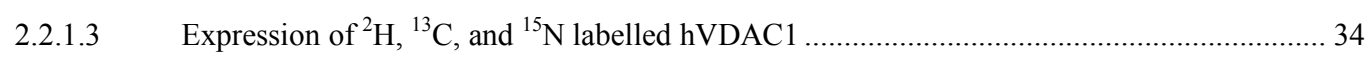

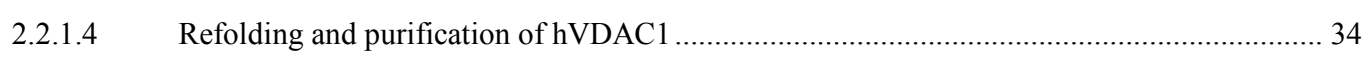




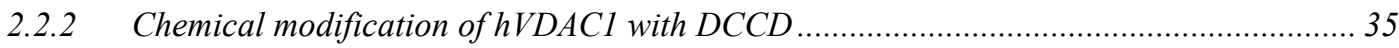

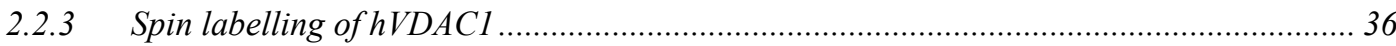

2.2.4 Bilayer measurements of hVDAC1 preparations ..................................................... 36

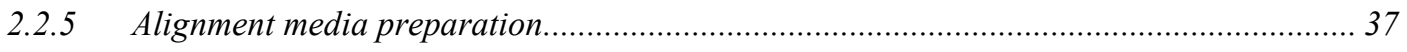

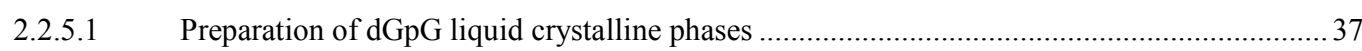

2.2.5.2 Preparation of Pf1 phage liquid crystalline phases ............................................................... 37

2.2.5.3 Preparation of charged polyacrylamide gels ..................................................................... 38

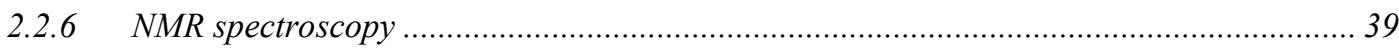

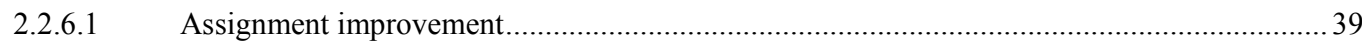

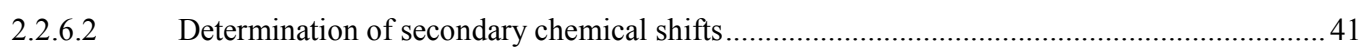

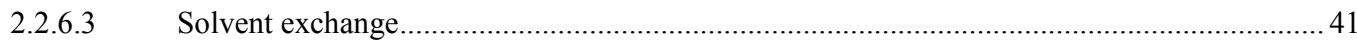

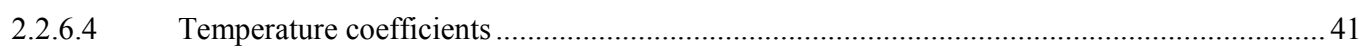

2.2.6.5 Steady state heteronuclear $\left\{{ }^{1} \mathrm{H}\right\},{ }^{15} \mathrm{~N}$-nuclear Overhauser effects (NOEs) ........................... 41

2.2.6.6 Peak intensities as indicators for chemical exchange ......................................................... 42

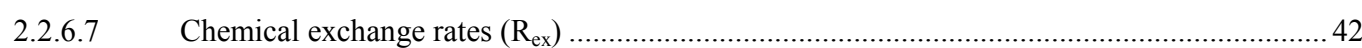

2.2.6.8 Estimation of $\mathrm{T} 2$ and the global rotational correlation time ..................................................... 44

2.2.6.9 Determination of residual ${ }^{2} \mathrm{H}$ quadrupole splitting .......................................................... 45

2.2.6.10 RDC measurements and evaluation with PALES ............................................................ 45

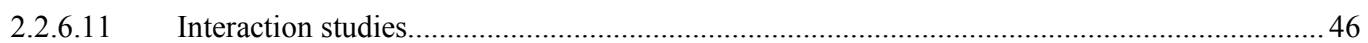

2.2.7 Gaussian Network Model Analysis........................................................................... 48

2.2.8 Electrostatic potential calculations.............................................................................. 48

2.2.9 MD simulations and principal component analysis (PCA) ............................................ 48

3 RESULTS

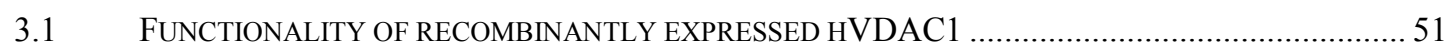

3.2 IMPROVING THE STRUCTURE OF HVDAC1 AND THE ORIENTATION OF ITS N-TERMINAL HELIX 53

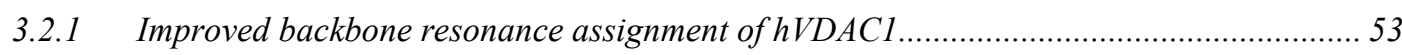

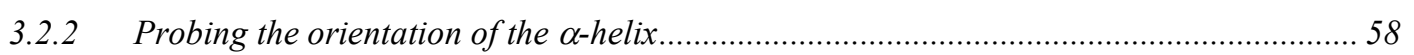

3.2.3 Helix destabilization affects hVDAC1 in a complex manner.............................................. 60

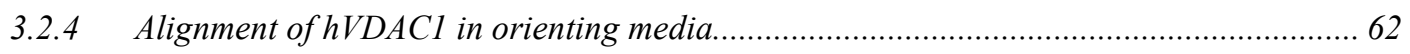

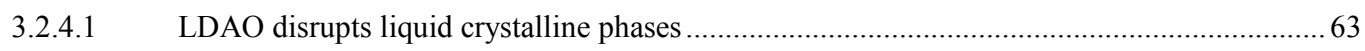

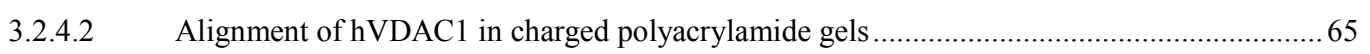

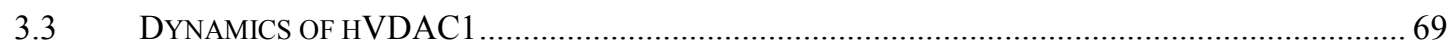

3.3.1 Conformational instability of the N-terminal part of hVDAC1 probed by H/D exchange. 69

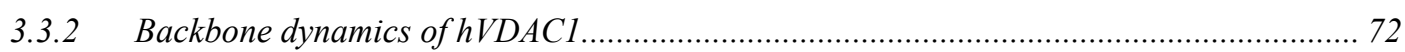

3.3.3 Increased B-factors in the crystal structure of VDAC correlate with low-frequency modes derived from Gaussian network model analysis ...................................................................... 77

3.3.4 The single-point mutation E73V and the reaction of E73 with DCCD stabilize the hVDAC1

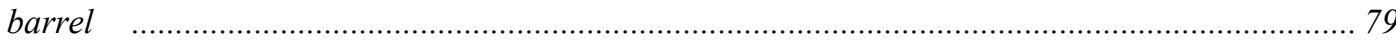

3.3.5 Reduced oligomerization of $h V D A C 1$ is not the main cause for reduced dynamics in the $N$ terminal barrel region 
3.3.6 VDAC1 dynamics: Insight from molecular dynamics studies ..................................... 84

3.3.6.1 VDAC flexibility from MD simulations and the role of charge on E73 ............................... 84

3.3.6.2 A negative charge on the side chain of E73 leads to thinning of the nearby membrane ......... 86

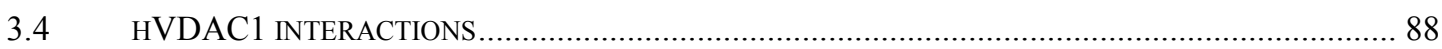

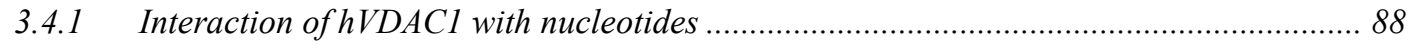

3.4.1.1 ATP interacts with hVDAC1 at several sites ............................................................... 88

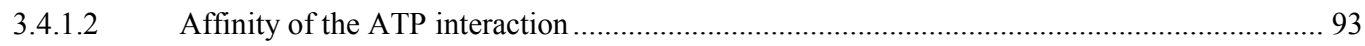

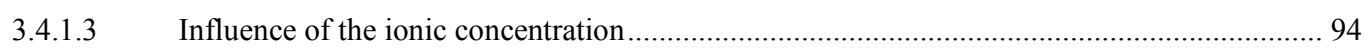

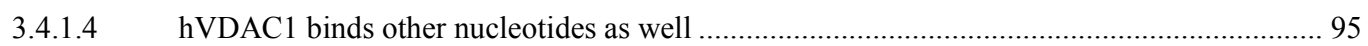

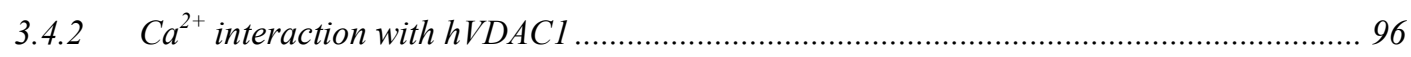

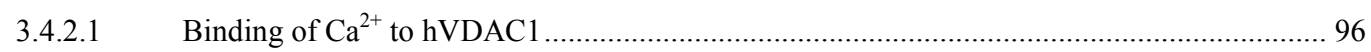

3.4.2.2 Lanthanide ions confirm the $\mathrm{C}$-terminal $\mathrm{Ca}^{2+}$-interacting region ...................................... 100

3.4.2.3 Interaction with ruthenium red (RuR) confirms $\mathrm{Ca}^{2+}$ interacting regions ........................... 101

3.4.2.4 $\mathrm{Ca}^{2+}$ influence on VDAC conductivity ............................................................................ 103

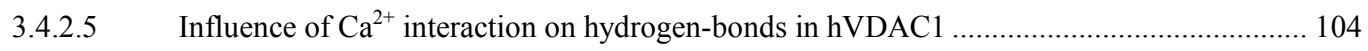

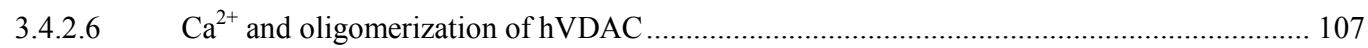

4 DISCUSSION

4.1 THE STRUCTURE OF HVDAC1 REPRESENTS THE NATIVE CONFORMATION........................... 109

4.2 DYNAMICS IN THE HVDAC1 BARREL ON A WIDE TIME SCALE ........................................... 110

4.2.1 The role of charge on E73 for VDAC1 dynamics....................................................... 112

4.2.2 Functional aspects of VDAC1 dynamics..................................................................... 113

4.3 STRUCTURE AND DYNAMICS OF THE N-TERMINAL HELIX IN HVDAC1 .............................. 115

4.3.1 Ambivalence of the N-terminal helix: barrel stabilization and dynamics ....................... 115

4.3.2 Cross-talk of dynamics in the $\beta$-barrel and the $\alpha$-helix ........................................... 117

4.3.3 Models for VDAC1 gating involving the $\alpha$-helix ................................................... 117

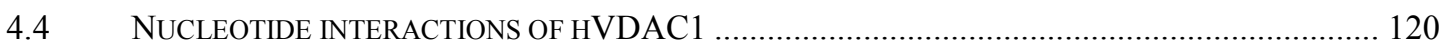

4.4.1 VDAC possesses a nucleotide binding site encompassing the helix-to-barrel linker....... 120

4.4.2 Nonspecific ionic influences in the N-terminal barrel half of VDAC ........................... 122

4.4.3 Affinity, selectivity, and transport of ATP ................................................................ 123

4.4.4 VDAC possesses a common nucleotide binding site ....................................................... 125

4.4.5 Implications of ATP binding for VDAC gating .................................................... 126

4.4.6 VDAC-ATP-hexokinase: a model................................................................................. 127

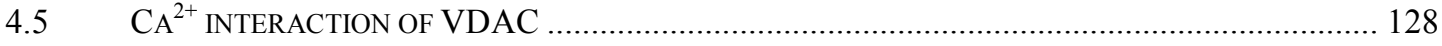

4.5.1 VDAC possesses two $\mathrm{Ca}^{2+}$ binding sites ............................................................... 128

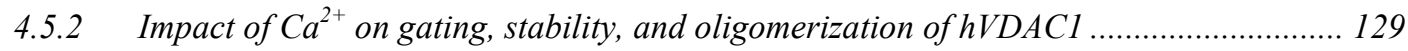

4.5.3 Interplay of $\mathrm{Ca}^{2+}$ interaction, dynamics, and oligomerization...................................... 131 


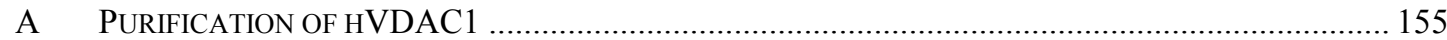

A.1 Immobilized metal ion affinity chromatography (IMAC) ........................................ 155

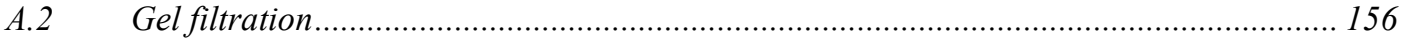

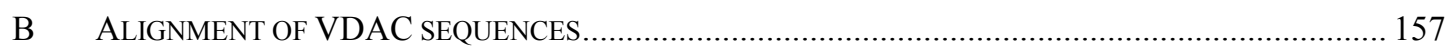

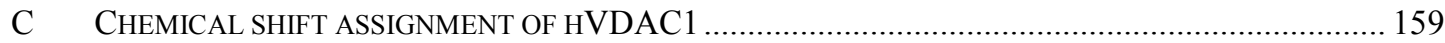

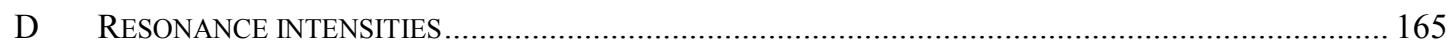

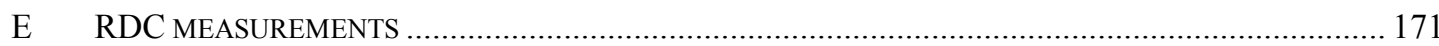

E.1 Estimation of accuracy of the "RDC-TROSY" experiment for measurements of ${ }^{1} \mathrm{H}_{-}{ }^{15} \mathrm{~N}$

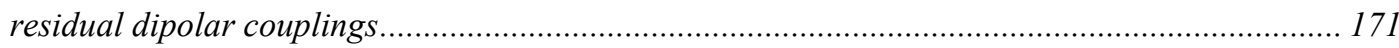

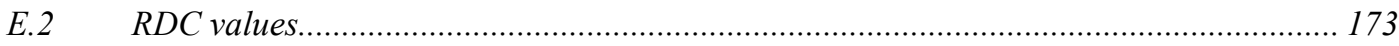

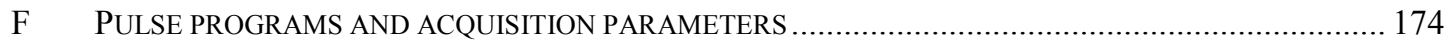

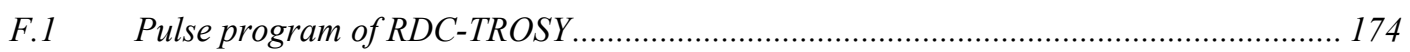

F.2 Acquisition parameters for RDC-TROSY measured on an isotropic sample of wt hVDAC1.

F.3 Pulse program for measurement of chemical exchange rates contributing to ${ }^{15} \mathrm{~N}$ transverse

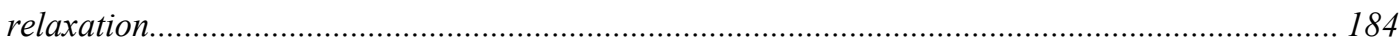

F.4 Acquisition parameters for chemical exchange rates measured on wt hVDAC1 ............ 188 


\section{Abbreviations}

$\begin{array}{ll}\text { AMPS } & \text { 2-acrylamido-2-methyl-1-propanesulfonic acid } \\ \text { ANT } & \text { adenine nucleotide translocase } \\ \text { APS } & \text { ammonium persulfate } \\ \text { APTMAC } & \text { (3-acrylamidopropyl)-trimethylammonium chloride } \\ \text { ATP } & \text { adenosinetriphosphate } \\ \text { CPMG } & \text { Carr-Purcell-Meiboom-Gill } \\ \text { dGpG } & \text { 2'-deoxyguanylyl(3' } \rightarrow \text { 5')-2'-deoxyguanosine } \\ \text { DCCD } & \text { N,N'-dicyclohexylcarbodiimide } \\ \text { DHPC } & \text { 1,2-dihexanoyl-sn-glycero-3-phosphocholine } \\ \text { DMPC } & \text { dimyristoylphosphatidylcholine } \\ \text { DPC } & \text { dodecylphosphocholine } \\ \text { DTT } & \text { dithiothreitol } \\ \text { E. coli } & \text { Escherichia coli } \\ \text { EDTA } & \text { ethylenediaminetetraacetic acid } \\ \text { GNM } & \text { Gaussian network model } \\ \text { HTP } & \text { guanosinetriphosphate } \\ \text { hetNOE } & \text { heteronuclear nuclear Overhauser effect } \\ \text { heteronuclear single quantum correlation spectroscopy }\end{array}$


LDAO lauryl-dimethylamine-N-oxide

MD molecular dynamics

MgATP magnesium complexed ATP

MTSL (1-oxy-2,2,5,5-tetramethyl-3-pyrroline-3-methyl)-methanethiosulfonate

mVDAC1 murine voltage-dependent anion channel 1

ncVDAC Neurospora crassa voltage-dependent anion channel

NMR nuclear magnetic resonance

NOE nuclear Overhauser effect

PCA principal component analysis

$\mathrm{R}_{\mathrm{ex}} \quad$ chemical exchange rate constant

RMSF root-mean-square fluctuation

scVDAC Saccharomyces cerevisiae voltage dependent anion channel

TROSY transverse relaxation optimized spectroscopy

UTP uridinetriphosphate

VDAC voltage-dependent anion channel

wt wild type 


\section{Introduction}

\subsection{Mitochondria}

\subsubsection{Mitochondria and energy metabolism}

Mitochondria are responsible for most of the energy production in eukaryotic cells by oxidative phosphorylation (as reviewed in (Saraste 1999)). Moreover, the organelle controls other metabolic and signalling pathways and regulates metabolism under many pathological conditions (Lemasters and Holmuhamedov 2006). Mitochondria are separated from the cytoplasm by an outer and inner mitochondrial membrane (OMM/IMM). The IMM contains the respiratory chain complexes that use NADH produced by nutrient oxidation to generate a proton-gradient across the IMM (inner membrane potential), which in turn drives the production of ATP (Saraste 1999). Furthermore, the IMM hosts a variety of integral membrane proteins that mediate the exchange of metabolites across the IMM, such as ATP/ADP exchange via the adenine nucleotide translocase (ANT) - also termed ADP/ATP carrier (Pfaff and Klingenberg 1968) (as reviewed in (Klingenberg 2008)).

Four integral membrane protein families have been found in the OMM to date. Three OMM proteins are $\beta$-barrel forming components of large protein complexes: the translocase of the outer membrane (TOM complex), the sorting and assembly machinery (SAM complex), and the mitochondrial distribution and morphology (Mdm) complex (Hill et al. 1998; Meisinger et al. 2007; Meisinger et al. 2004; Wiedemann et al. 2003). Together with complexes in the IMM, these three OMM complexes mediate the translocation and insertion of nearly all synthesized proteins into mitochondria (for a 
review see (Bolender et al. 2008)). The fourth and most abundant $\beta$-barrel protein family is termed voltage-dependent anion channels (VDACs) due to their anion selectivity and voltage-dependent channel closure (Blachly-Dyson and Forte 2001; Schein et al. 1976). VDAC channels cover up to $80 \%$ of the OMM area in high density regions (Goncalves et al. 2007) and form various oligomers (Guo et al. 1995; Hoogenboom et al. 2007; Mannella 1982) (Figure 1). VDAC mediates the flux of all metabolites and ions across the OMM (Benz 1994). Together, this small number of protein families mediates the entire communication between mitochondria and the cytosol (Benz 1994; Bolender et al. 2008).
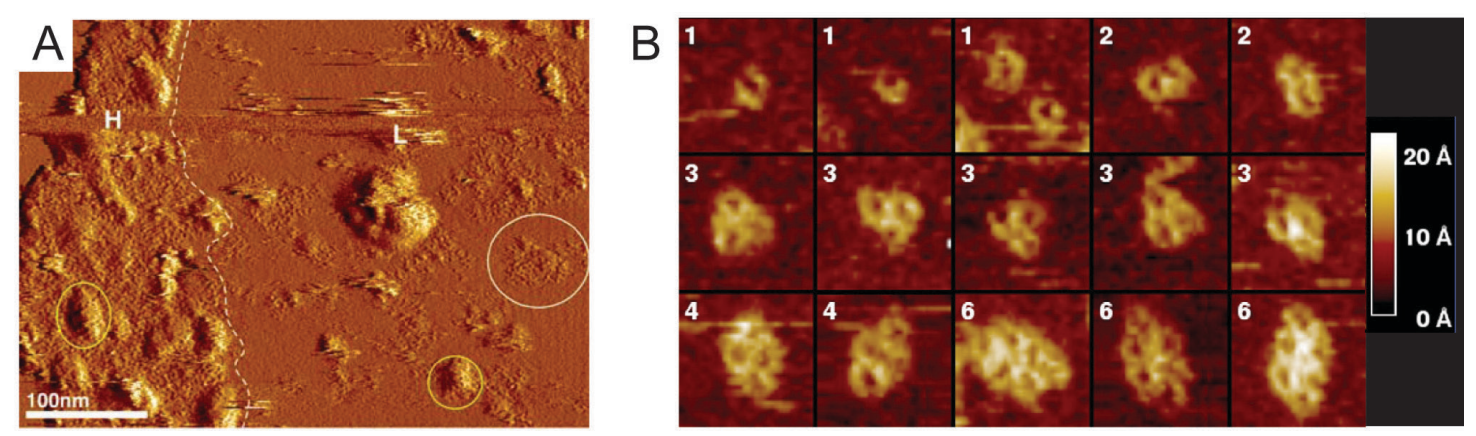

Figure 1. AFM images of the native OMM. (A) AFM image of a Saccaromyces cerevisiae (sc) OMM patch containing low-density (L) and high-density (H) scVDAC domains. Protein corrugation (white outline) is easily distinguishable from smooth lipid areas. Large protrusions (yellow outline) underlie VDAC in both low-density and high-density domains. Reprinted from (Goncalves et al. 2007), Copyright (2007), with permission from Elsevier. (B) Gallery of VDAC oligomeric states observed from highresolution frequency modulation AFM topographs of the potato tuber mitochondria OMM. The various oligomeric states of VDAC are marked. The frame size of the gallery figures is $210 \AA$. Reprinted from (Hoogenboom et al. 2007), Copyright (2007), with permission from Elsevier.

\subsubsection{Mitochondrial role in cell death}

Mitochondria also mediate necrotic and apoptotic cell death (Crompton 1999; Ravagnan et al. 2002). Apoptosis or programmed cell death enables organisms to control tissue homeostasis and is required for embryonic and immune system development. Furthermore, many pathological conditions, such as infection, UV damage, stroke, heart attack, cancer, and neurodegenerative diseases are related to increased or insufficient apoptosis. Apoptotic pathways result in the activation of caspases, restriction enzymes that execute the apoptotic program (as reviewed in (Hengartner 2000)).

The mitochondrial apoptotic pathway is stimulated by intracellular stress signals, such as mitochondrial $\mathrm{Ca}^{2+}$ overload, elevated levels of reactive oxygen species (ROS), 
staurosporine, chemotherapy, and DNA damage, and results in mitochondrial outer membrane permeabilization (MOMP) (for a review see (Tait and Green 2010)). MOMP manifests in the release of cytochrome $\mathrm{c}$ and other pro-apoptotic proteins into the cytosol (Liu et al. 1996; Susin et al. 1996). Once released, the pro-apoptotic effectors induce apoptosome formation and activation of downstream caspases (Hengartner 2000). Regulation of apoptosis is mediated by pro- and anti-apoptotic members of the Bcl-2 family (as reviewed in (Adams and Cory 1998; Tsujimoto and Shimizu 2000)). Despite extensive research, the mechanism of MOMP is still a matter of debate. Diverging models suggest that MOMP is caused either by a specific protein channel, by non-specific membrane rupture following mitochondrial swelling, or by lipidic pores (Feldmann et al. 2000; Grimm and Brdiczka 2007; Tait and Green 2010; and references therein). Due to its high abundance in the OMM, either process might involve VDAC. 


\subsection{Membrane proteins}

About $20 \%$ of the proteome is composed of membrane proteins (Schulz 2002). While soluble protein folds exhibit a high variability, the topology of integral membrane proteins is limited to two categories: $\alpha$-helical and $\beta$-barrel proteins. $\alpha$-helical membrane proteins are found in the plasma membrane and all inner membranes (endoplasmic reticulum, peroxisomes, chloroplasts, mitochondria and bacteria). They are involved in transport across membranes, cell signalling, metabolism and regulation and are intensively addressed targets for drug discovery (Russell and Eggleston 2000). In contrast, outer membranes of mitochondria (and chloroplasts) and Gram-negative bacteria contain mainly or exclusively $\beta$-barrel membrane proteins, respectively (Schulz 2002). A role of bacterial $\beta$-barrel outer membrane proteins (OMPs) in antibiotic uptake and drug resistance has recently directed some attention to this class of membrane proteins (Delcour 2009). Increasing evidence for the regulatory role of VDAC in apoptosis and cancer inspired drug discovery processes for this $\beta$-barrel membrane protein (Arbel and Shoshan-Barmatz 2010; Galluzzi et al. 2008; Simamura et al. 2008).

\subsubsection{Membrane protein structures and interactions}

Structural information of (membrane) proteins supports functional understanding and drug discovery. However, both nuclear magnetic resonance (NMR) spectroscopy and $\mathrm{X}$-ray crystallography experience difficulties in structure determination of membrane proteins, reflected by the low number of known membrane protein structures to date: Currently, 285 unique membrane protein structures are known (membrane protein databank, http://www.mpdb.tcd.ie/; status of November, 2011), about 60 of which belong to OMPs (for an overview see (Fairman et al. 2011)). Although X-ray crystallography is the most common technique applied to date, ongoing advancement in solution NMR spectroscopy (high-field spectrometers, cryogenic probes, transverse relaxation optimized spectroscopy (TROSY) (Pervushin et al. 1997) in combination with deuteration (Salzmann et al. 1998), selective methyl-protonation (Tugarinov and Kay 2003), selective labelling schemes (Parker et al. 2004)) and the use of long-range structural information from paramagnetic relaxation enhancement (PRE) (Gillespie and 
Shortle 1997), pseudocontact shifts (PCS) (Hus et al. 2000) and residual dipolar couplings (RDCs) (Tjandra and Bax 1997; Tolman et al. 1995) facilitate the investigation of membrane protein structures embedded in micelles or bicelles. Currently, 30 membrane protein structures have been solved by NMR (membrane protein databank, http://www.mpdb.tcd.ie/; for recent overviews see (Nietlispach and Gautier 2011; Wang 2008)). $\beta$-barrel membrane proteins proved to be more amenable for NMR investigation due to their inherent stability, feasibility of functional refolding (Tamm et al. 2004; Wang 2008) and increased signal dispersion. Recent examples of NMR-derived membrane protein structures are the polytopic $\alpha$-helical diacylglycerol kinase (DAGK) (Van Horn et al. 2009) and sensory rhodopsin (pSRII) (Gautier et al. 2010), as well as the bacterial $\beta$-barrel proteins KpOmpA (Renault et al. 2009) and OprH (Edrington et al. 2011).

Membrane protein functions can often be deduced from crystal structures. This is especially valid when substrates or solutes are bound as in the sugar specific bacterial porins (Forst et al. 1998; Schirmer et al. 1995). NMR spectroscopy provides a large repertoire of methods for the study of interactions and is especially valuable when cocrystallization with ligands fails. For instance, NMR spectroscopy detected a ligand binding site in the $\alpha$-helical DsbB (Zhou et al. 2008) differing from the X-ray structure (Inaba et al. 2006) and revealed the binding site and orientation of adamantine drugs in the influenza M2 transmembrane domain with possible implications for improved drug design (Cady et al. 2010; Cady et al. 2011). For $\beta$-barrel membrane proteins NMR spectroscopy elucidated the lipopolysaccharide interaction of OprH (Edrington et al. 2011) and protein-micelle or protein-bicelle interactions of OmpX (Fernandez et al. 2002; Lee et al. 2008).

\subsubsection{Dynamics of membrane proteins}

${ }^{1}$ Protein structures provide a hint on functions such as ligand interactions. However, proteins are flexible at ambient temperature and their structures are time-averaged ensembles of spatially distinct states arising from motion on time scales of nanoseconds

\footnotetext{
${ }^{1}$ Section 1.2.2 contains some text passages from our own publication (Villinger et al. 2010).
} 
to hours. Importantly, protein dynamics provide an essential link between structure and function. Dynamics influence ligand interactions (via induced fit or conformational selection), catalysis, allostery, and ligand induced enzyme inhibition. Furthermore, dynamics influence signalling pathways, ion channel gating, protein folding, and misfolding (Kleckner and Foster 2011). In terms of dynamic characterization, X-ray crystallography is limited to the analysis of Debye-Waller- ("B"-) factors and the detection of ligand-stabilized structural rearrangements, for instance occurring during activation of the potassium channel Kir2.2 (Hansen et al. 2011). In contrast, NMR spectroscopy is a versatile experimental method for the study of protein dynamics from nanoseconds to seconds with atomic resolution (Kleckner and Foster 2011). In addition, when a $3 \mathrm{D}$ structure is available, insight into fast dynamics of proteins that occur on the nanosecond time scale might be obtained from molecular dynamics (MD) simulations. Course grained methods, such as elastic network models (Bahar et al. 1997; Tirion 1996), provide insight into collective motions on the nanosecond and longer time scales and can therefore complement the NMR results.

Membrane protein dynamics are gaining more attention since an increasing number of high-resolution structures are available. For the study of membrane protein dynamics, solution and solid state NMR spectroscopy proved increasingly valuable in the recent decade (for an overview see the special issue (Veglia and Ramamoorthy 2010) and recent reviews (Chill and Naider 2011; Hong et al. 2011)). NMR studies revealed large conformational changes essential for the physiological function of $\alpha$ helical membrane proteins, such as the interaction of phospholamban with effectors modulating heart muscle contractility (Chu et al. 2010; Traaseth and Veglia 2010), antimicrobial peptides adopting different conformations in membranes or solution (Bhattacharjya and Ramamoorthy 2009; Salnikov et al. 2010), and conformational changes in the selectivity filter of the potassium channel KcsA that determine its gating properties (Imai et al. 2011).

Motions of outer membrane $\beta$-barrels are less well characterized. A pioneering solution NMR study revealed $\mu \mathrm{s}-\mathrm{ms}$ interconversion of the catalytic loop of PagP between an excited and a non-excited state, enabling both ligand binding and enzymatic catalysis (Hwang and Kay 2005). Non-enzymatic $\beta$-barrel channels exhibit more 
general dynamic features, such as loop flexibility and slow conformational exchange towards the extracellular barrel edges, indicating relevance for immunogenicity of small monomeric OMPs (Arora et al. 2001; Liang et al. 2010; Renault et al. 2009) and pH dependent gating of the OmpG porin (Liang and Tamm 2007; Yildiz et al. 2006). 


\subsection{The voltage-dependent anion channel (VDAC)}

\subsubsection{General characteristics and isoforms}

VDAC, also termed mitochondrial porin, was initially described as reminiscent of bacterial porins due to its pore-forming activity in bilayer membranes (Schein et al. 1976). The $30-32 \mathrm{kDa}$ protein of roughly 285 amino acids forms water filled diffusion pores of 2-3 nm diameter (Mannella 1982) that are permeable for molecules up to 3 $6 \mathrm{kDa}$ (Benz 1994). Mitochondria from yeast contain one or two VDAC isoforms, while up to three isoforms with tissue-specific expression levels have been found in mammals (Buettner et al. 2000; Rahmani et al. 1998; Sampson et al. 1997). The first discovered mammalian isoform, VDAC1, is the best characterized and most abundant isoform (De Pinto et al. 2010). VDAC1 and VDAC2 display strong pore-forming activity in bilayer membranes, while VDAC3 is less active (Xu et al. 1999). The majority of VDAC channels from various species exhibits very similar conductance, selectivity, and voltage-gating as VDAC1 from mammals (Colombini 1989).

\subsubsection{Conductance, selectivity, and gating of VDAC}

When reconstituted in planar phospholipid bilayers, VDAC displays high conductivity (4 ns in $1 \mathrm{M} \mathrm{KCl}$ ) at low voltages (within \pm 20 to $\pm 30 \mathrm{mV}$ ) (Benz 1994). In this highconductance "open" state, VDAC is slightly anion selective $\left(2: 1 \mathrm{Cl}^{-} / \mathrm{K}^{+}\right)$(Colombini 1989) and permeable to large anions like ATP (Rostovtseva and Colombini 1997) (Figure 2). A variety of charged residues in the pore lumen, responsible for the ion selectivity, were identified by mutational studies (Blachly-Dyson et al. 1990; Peng et al. 1992). At voltages above $\pm 20 \mathrm{mV}$ VDAC undergoes a conformational change to a variety of low-conductance "closed" states. Channel closure leads to a $50-80 \%$ reduction in conductivity (Benz 1994; Schein et al. 1976), consistent with a smaller pore diameter (Colombini et al. 1987; Zimmerberg and Parsegian 1986). The closed states of VDAC favour small cations (Benz et al. 1990; Colombini 1980) and are impermeable to ATP (Rostovtseva and Colombini 1997) (Figure 2). 


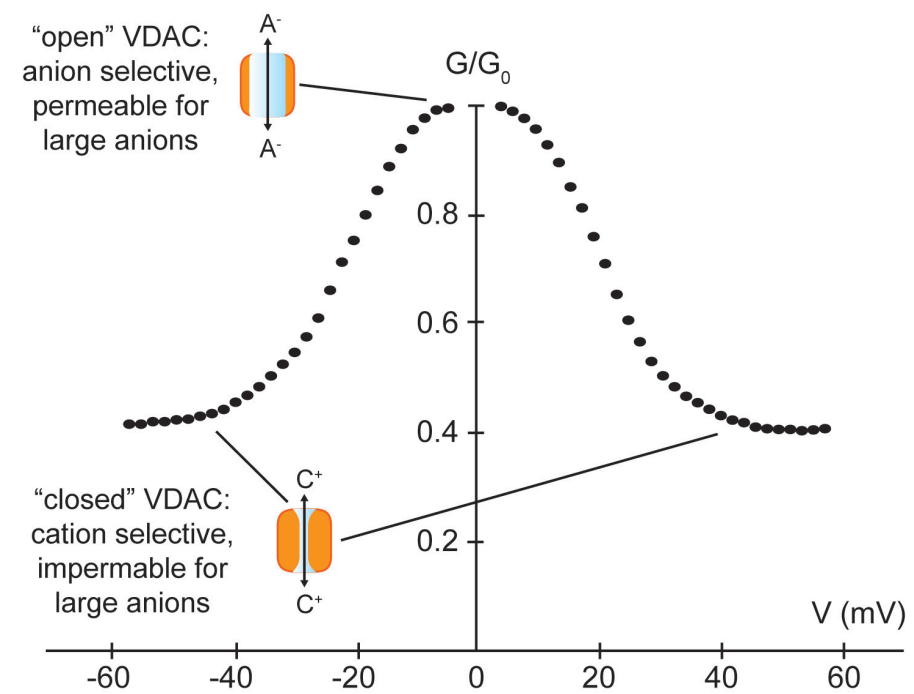

Figure 2. Voltage gating of VDAC in bilayer membranes. Schematic representation of the steady-state conductance $(\mathrm{G})$ at any given voltage compared to the open conductance at low voltage $\left(\mathrm{G}_{0}\right)$ of multiple VDAC channels in lipid bilayer membranes. The amount of reduction in conductance is buffer dependent and an arbitrary level is shown here. Changes of conformation and selectivity of VDAC are indicated for the two extreme states.

This characteristic resulted in the widely accepted opinion that VDAC regulates metabolite flux across the OMM. Although voltage gating occurs at much lower voltages than for bacterial OMPs $(\sim 100 \mathrm{mV})$ (Liu and Delcour 1998; Saxena et al. 1999; Schulz 2000), the existence of a membrane potential across the OMM is unclear, and the physiological impact of voltage-gating is debated (Benz 1994; Colombini 2004). However, VDAC gating is also modulated by a variety of small molecules and proteins (as reviewed in (Shoshan-Barmatz et al. 2006)), suggesting another physiological significant mechanism. Two important modulators of VDAC conductance are hexokinase and NADH that induce channel closure and inhibit ATP flux across the OMM (Azoulay-Zohar et al. 2004; Lee et al. 1994; Perevoshchikova et al. 2010). However, the main conductance states of VDAC in native membranes are controversial (Kinnally et al. 1987; Moran et al. 1992; Wunder and Colombini 1991).

\subsubsection{VDAC's role in metabolism and apoptosis}

Today the highly abundant VDAC channel is regarded as a key regulator of mitochondrial function and cellular energy metabolism (Lemasters and Holmuhamedov 2006) (Figure 3). In complex with hexokinase, mitochondrial creatine kinase, and ANT, VDAC couples cytosolic glycolysis to mitochondrial oxidative phosphorylation rates (Brdiczka 1991; Grimm and Brdiczka 2007). VDAC is also involved in $\mathrm{Ca}^{2+}$ signalling 
between the endoplasmic reticulum and mitochondria (Rapizzi et al. 2002) and has been connected to abnormal metabolic regulation. For instance, increased expression levels of mitochondria-bound hexokinase isoforms in tumor cells (Bustamante et al. 1981) and hexokinase-induced VDAC channel closure (Azoulay-Zohar et al. 2004) suggest that VDAC regulation is responsible for the Warburg effect (aerobic glycolysis) in cancer cells (Lemasters and Holmuhamedov 2006). Furthermore, VDAC overexpression and silencing affect ATP production and cell growth (Abu-Hamad et al. 2006), in line with high VDAC expression levels observed in energy demanding tumor cells (Simamura et al. 2008).

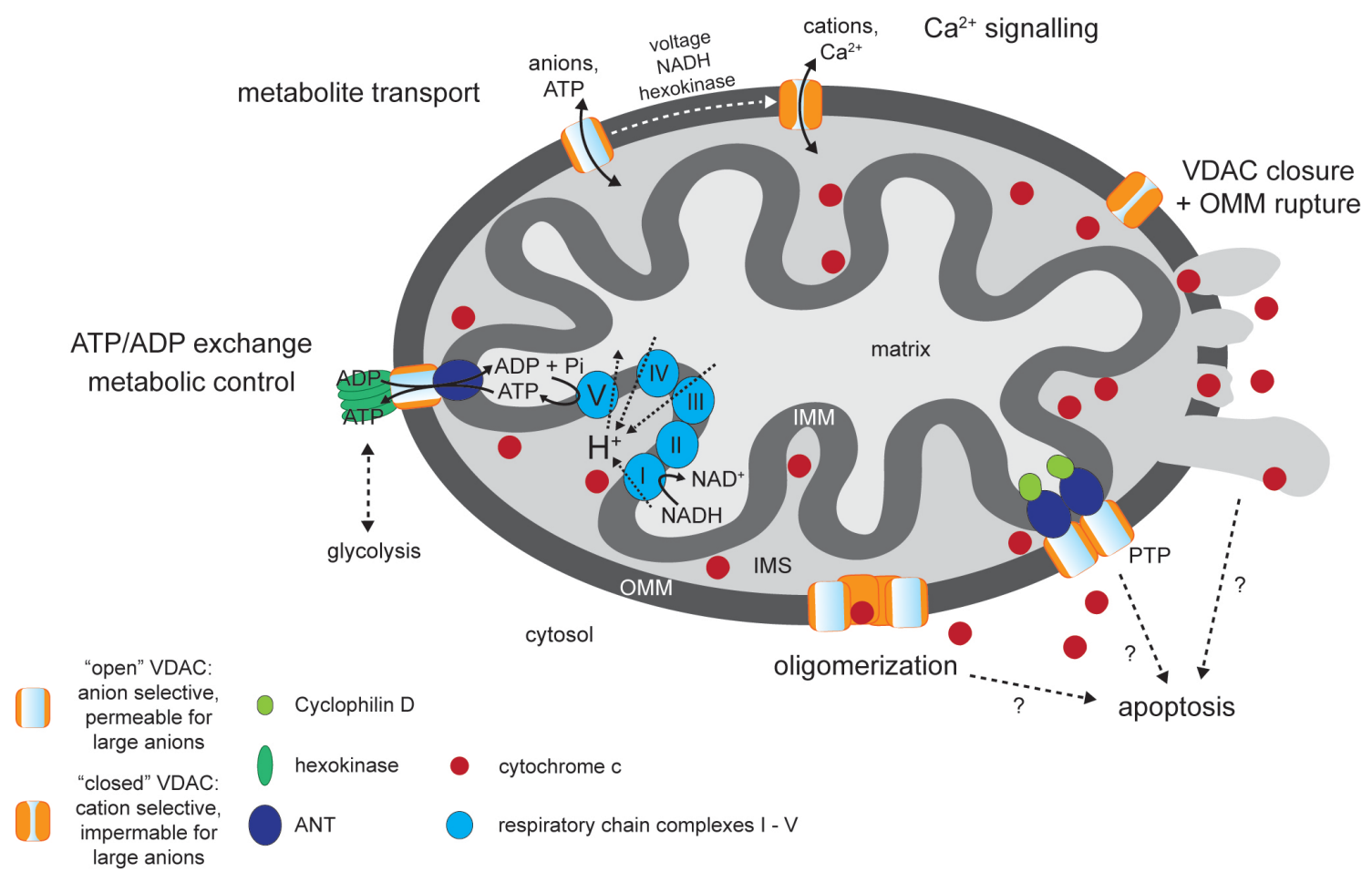

Figure 3. VDAC mediated regulation of metabolism and apoptosis. For a description see main text.

Increasing evidence suggests a role of VDAC in apoptosis (for a review see (Shoshan-Barmatz et al. 2010a)). VDAC specific antibodies inhibit $\mathrm{Ca}^{2+}$-induced MOMP (Shimizu et al. 2001). Furthermore, overexpression of VDAC results in increased apoptosis (Zaid et al. 2005), while RNAi silencing prevents apoptosis (Tajeddine et al. 2008). In addition, ruthenium red (RuR) and hexokinase interaction with VDAC induce channel closure and prevent apoptosis (Azoulay-Zohar et al. 2004; Pastorino et al. 2002; Zaid et al. 2005). Many Bcl-2 family proteins induce changes in VDAC permeability and cytochrome $\mathrm{c}$ release, resulting in a large number of proposals 
for the involvement of VDAC in MOMP (Shoshan-Barmatz et al. 2010a). Among these models are (i) protein pores composed of VDAC homo-oligomers (Keinan et al. 2010; Zheng et al. 2004) or hetero-oligomers (Shimizu et al. 2000), (ii) VDAC as part of the $\mathrm{Ca}^{2+}$ - and Bcl-2 family regulated permeability transition pore (PTP) (Narita et al. 1998; Szabo et al. 1993; Zoratti and Szabo 1995), and (iii) Bcl-2 regulated VDAC closure that leads to inhibition of metabolite exchange, osmotic swelling, and non-specific membrane rupture (Rostovtseva et al. 2004; Vander Heiden et al. 1999; Vander Heiden et al. 2001) (Figure 3).

\subsubsection{The structure of VDAC1}

Early structural models of VDAC derived from computational predictions and biochemical data claimed the formation of 12 (Blachly-Dyson et al. 1990), 13 (Song et al. 1998b), 16 (Casadio et al. 2002), 18 (Al Bitar et al. 2003), or 19 (Forte et al. 1987) $\beta$-strands. Furthermore, an amphipathic N-terminal $\alpha$-helix was part of all structural models and its structure was determined in SDS- and TFE- solution (De Pinto et al. 2007). Various studies suggested the helix to be part of the pore (Colombini 2004), exposed to the cytosol (De Pinto and Palmieri 1992), or lie on the membrane surface (Guo et al. 1995; Mannella 1998). The location of the helix and the barrel architecture were recently resolved by three high-resolution structures of mammalian VDAC1. Simultaneously to our published 3D structure of hVDAC1 (Figure 4A), determined by a combination of NMR spectroscopy and X-ray crystallography (Bayrhuber et al. 2008), the structures of hVDAC1 (Hiller et al. 2008) and murine VDAC1 (mVDAC1) (Ujwal et al. 2008) have been determined separately by NMR spectroscopy and X-ray crystallography, respectively. All three structures demonstrate a location of the helix inside the pore of a 19 -stranded $\beta$-barrel (Figure 4). The odd number of $\beta$-strands, resulting in the parallel arrangement of $\beta$-strands $\beta 1$ and $\beta 19$, distinguishes VDAC from bacterial OMPs with even $\beta$-strand numbers ranging between 8 and 24 (Fairman et al. 2011; Schulz 2002). All three structures also revealed an unusual position of a glutamic acid residue, E73, in the middle of $\beta 4$ and exposed to the hydrophobic environment (Figure 4A). 


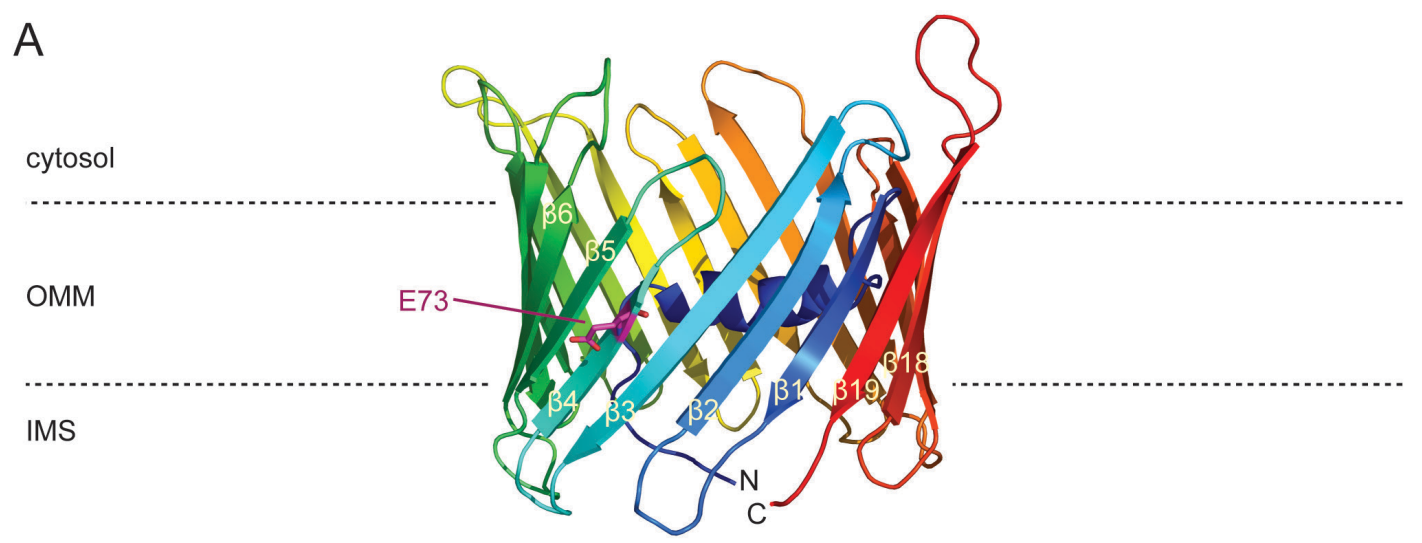

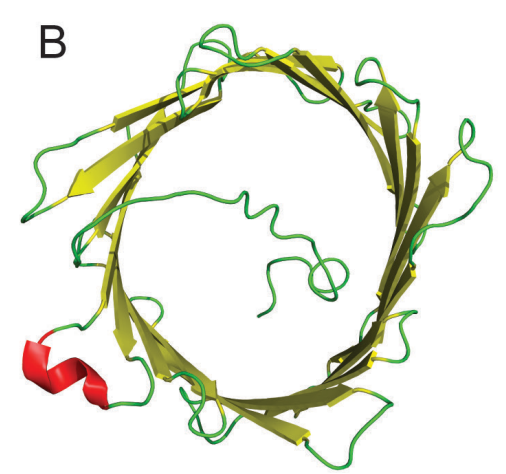

hVDAC1

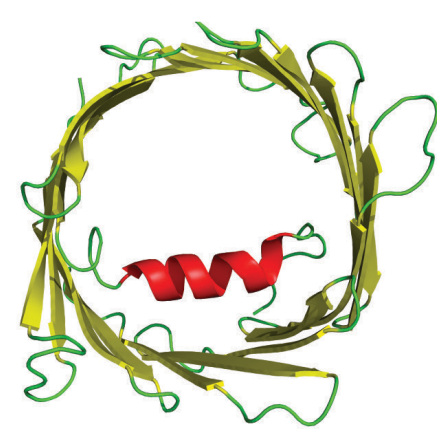

hVDAC1 $\mathrm{NMR}+\mathrm{X}$-ray

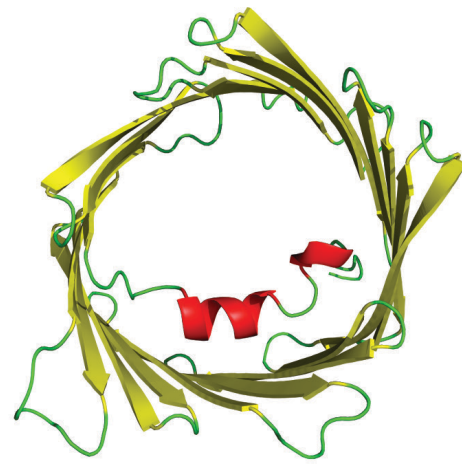

mVDAC1

X-ray

Figure 4. 3D structures of human and murine VDAC1. (A) Combined NMR/X-ray structure of hVDAC1 (PDB code: 2JK4) (Bayrhuber et al. 2008) shown from a front view, displaying the parallel strand arrangement of $\beta 1$ and $\beta 19$ and the location of E73 in $\beta 4$ (purple sticks). The structure is displayed in a cartoon representation colour-coded from the N-terminus (blue) to the C-terminus (red). The approximate position of the surrounding OMM is indicated by dotted lines. The suggested cytosolic and inter membrane space (IMS) sites are indicated (Bayrhuber et al. 2008). (B) Comparison of the combined hVDAC1 structure with the NMR structure of hVDAC1 (PDB code: 2K4T) (Hiller et al. 2008) and the crystal structure of mVDAC1 (PDB code: 3EMN) (Ujwal et al. 2008). Helices are colored in red, $\beta$ strands in yellow and unstructured regions in green. All structures are displayed in cartoon representation generated with PyMOL (DeLano 2003).

While the 19-stranded $\beta$-barrel is very similar in all three high-resolution structures, the major structural difference appears in the location and orientation of the $\mathrm{N}$-terminal $\alpha$-helix (Figure 4B). In the crystal structure the $\alpha$-helix is kinked at residues 10-11 and binds tightly to the central $\beta$-strands, leaving a flexible linker of five residues $\left({ }^{21} \mathrm{GYGFG}^{25}\right)$ connecting the $\alpha$-helix to the first $\beta$-strand. A similar $\alpha$-helical structure of hVDAC1 in liposomes has been determined by solid state NMR (Schneider et al. 2010). In contrast, in the solution NMR structure (Hiller et al. 2008) the second part of the helix (residues 11-20) is unstructured, while our combined NMR/X-ray structure of 
hVDAC1 (Bayrhuber et al. 2008) revealed a full $\alpha$-helix without kink that is rotated by $\sim 90^{\circ}$ along its long axis with respect to the crystal structure of murine VDAC1. The observed differences in the orientation and length of the helix could be due to differences in resolution of the three structures or due to dynamics of the helix. This highlights the importance to study the structure and dynamics of hVDAC1 and in particular its $\mathrm{N}$-terminal $\alpha$-helix that might be involved in gating (Hiller and Wagner 2009; Ujwal et al. 2008) (see below).

\subsubsection{Interactions and metabolite transport}

With the known structure of VDAC1, interactions with ions, molecules, and proteins can be investigated at high resolution. For instance, the interaction sites of hVDAC1 with NADH, Bcl- $\mathrm{x}_{\mathrm{L}}$ and cholesterol have been determined by NMR spectroscopy (Hiller et al. 2008). VDAC specifically interacts with various nucleotides and other metabolites, while small non-physiological solutes do not penetrate the channel (Rostovtseva and Bezrukov 1998; Zizi et al. 1994). How this discrimination is accomplished is unclear. Furthermore, interactions with the most important metabolites exchanged by VDAC - ATP and ADP - have only been detected indirectly. Crosslinking and peptide interaction studies localized nucleotide interactions to the $\mathrm{N}$ terminal $\alpha$-helix/linker and $\beta$-strands $\beta 7$ and $\beta 18$ - $\beta 19$ (Florke et al. 1994; Yehezkel et al. 2007; Yehezkel et al. 2006). However, more detailed information on ATP binding and permeation are needed. NMR interaction studies allow detailed characterization of ATP binding and its effect on VDAC structure and dynamics.

Both the open state and the classical closed states of hVDAC1 are reported to bind and permeate $\mathrm{Ca}^{2+}$ (Gincel et al. 2001; Tan and Colombini 2007). In addition, contradictory reports dispute over the presence of a regulatory effect of $\mathrm{Ca}^{2+}$ on channel opening required for ATP transport (Bathori et al. 2006; Rostovtseva et al. 2005). Two $\mathrm{Ca}^{2+}$ binding sites have been indirectly deduced from bilayer measurements of VDAC mutants in the presence of $\mathrm{Ca}^{2+}$ or ruthenium compounds (Israelson et al. 2007; Israelson et al. 2008). However, direct evidence for the location of $\mathrm{Ca}^{2+}$ binding sites and their influence on the VDAC structure, which might shed light onto transport and gating mechanisms of VDAC, is missing. 


\subsubsection{Models for VDAC channel closure}

Regulation of metabolite transport by voltage (Blachly-Dyson and Forte 2001; Schein et al. 1976), small molecules, and proteins (Shoshan-Barmatz et al. 2006) is not fully understood. Residues involved in gating were mainly localized to the $\alpha$-helix and the Nterminal $\beta$-strands (Thomas et al. 1993). Similarly, residues in the helix and E73 in the $\mathrm{N}$-terminus were identified to be important for hexokinase interaction, channel closure and protection from apoptosis (Abu-Hamad et al. 2009; Zaid et al. 2005). To enable interactions and gating between various states, conformational variability of VDAC is expected. Indeed, large structural rearrangements have been proposed on the basis of electron microscopy (Guo and Mannella 1993) and bilayer measurements (Peng et al. 1992; Zimmerberg and Parsegian 1986). Based on electrophysiological data, the removal of a large transmembrane part - the positively charged "voltage-sensor" - out of the membrane was suggested to result in a smaller pore formed by the remaining $\beta$ strands (Song et al. 1998a; Thomas et al. 1993) (Figure 5A). Considering the 19stranded structure of VDAC, the voltage-sensor would comprise the $\alpha$-helix and $\beta$ strands $\beta 2-\beta 5, \beta 9-\beta 10$, and $\beta 19$. In line with the recent $3 \mathrm{D}$ structures it was proposed that gating involves merely motion of the N-terminal helix into the pore lumen without an influence on the $\beta$-barrel. The helix motion was suggested to be facilitated by the interaction of NADH and other modulators with $\beta$-strands $\beta 18$ and $\beta 19$, which would subsequently displace the linker and the N-terminal $\alpha$-helix (Hiller and Wagner 2009; Ujwal et al. 2008) (Figure 5B, C). In addition to this model of steric channel blockage, reorientation of the helix has been suggested to change the electrostatic potential in the pore (Shoshan-Barmatz et al. 2010a) and thus provide an electrostatic blockage for charged molecules. However, high-resolution information about these conformational changes and dynamics of the channel are missing. 
A
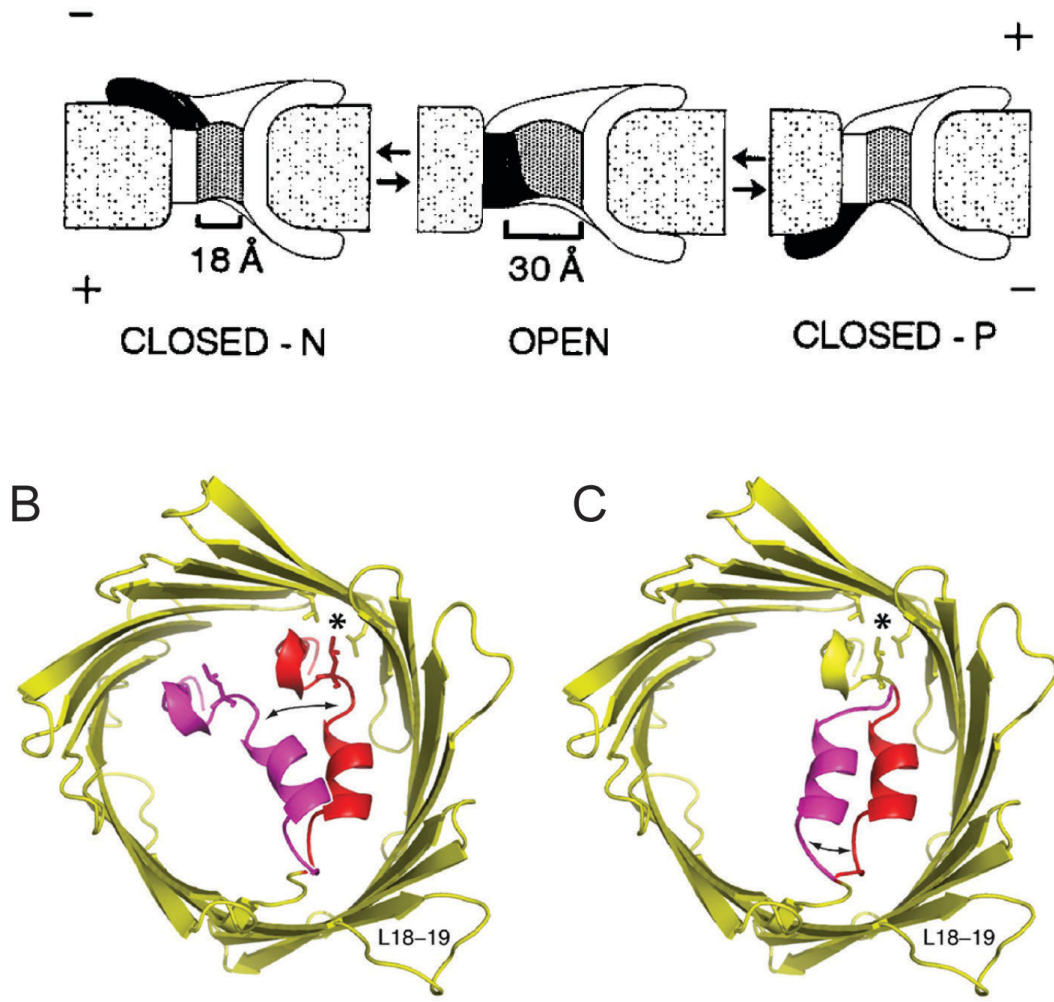

Figure 5. Proposed gating models for VDAC. (A) Motion of large transmembrane parts out of the pore proposed on the basis of biochemical and electrophysilogical data (Song et al. 1998a). Longitudinally bisected views of the open state (center) and the closed states achieved at positive (CLOSED-P) and negative (CLOSED-N) potentials. The sensor domain is indicated by the black region. In both gating processes, the sensor domain moves toward the negative side of the membrane. Reprinted from (Song et al. 1998a), Copyright (1998), with permission from Elsevier. (B, C) Gating models based on the highresolution structure of mVDAC1 (Ujwal et al. 2008). The structure of mVDAC1 is displayed as viewed perpendicular to the membrane. Barrel and loop residues are colored yellow, the hydrophobic contact between L10, V143, and L150 (sticks) is indicated by an asterisk. Loop L18-19, which is involved in NADH interaction, is labelled. The $\alpha$-helix in red or magenta represents the proposed open and closed state, respectively. (B) shows the model proposed by Ujwal et al. (Ujwal et al. 2008). Here, the complete helix (residues 1-20) undergoes a conformational change to the closed state. In (C) an alternative gating model is shown (Hiller and Wagner 2009), in which only residues 11-20 undergo conformational changes, preserving the hydrophobic contact $\left(^{*}\right)$. Reprinted from (Hiller and Wagner 2009), Copyright (2009), with permission from Elsevier. 


\subsection{Aims and Outline}

Increasing evidence indicates that flexibility and conformational changes occur in the $\beta$ barrel and the $\alpha$-helix of VDAC1. Since both regions are involved in protein interaction and gating, it is very likely that dynamic rearrangements influence these processes. In addition, the impact of $\mathrm{Ca}^{2+}$ and ATP on gating, as well as the precise interactions sites are unknown. Thus, the aim of this thesis is the characterization of VDAC1 dynamics and interactions in order to shed light on metabolite interactions, transport and gating.

The first part of this thesis aims at the improvement of the structure of the $\mathrm{N}$ terminal hVDAC1 $\alpha$-helix and the characterization of its structural changes under varying conditions in LDAO solution. For this purpose, the assignment of hVDAC1 is increased by improved sample purification and additional NMR spectra. Furthermore, solution NMR chemical shifts and intensities of mutant forms of hVDAC1 are investigated. Additionally, suitable alignment media for the measurement of RDCs are screened.

In the second part, the dynamics of VDAC1 are characterized by a combination of solution NMR and Gaussian network model (GNM) analysis. NMR spectroscopy is used to characterize ps-ns and $\mu$ s-ms dynamics, as well as hydrogen-bond stability of hVDAC1. The GNM analysis provides information about the intrinsic flexibility of murine VDAC1 (mVDAC1) observed in the crystal structure. Additional information about protein dynamics in the ps-ns time scale as well as lipid interactions are provided by $\mathrm{MD}$ simulations of the mVDAC1 crystal structure, performed in collaboration by Rodolfo Briones, Ulrich Zachariae, and Bert L. de Groot (Department of Theoretical and Computational Biophysics, Max Planck Institute for Biophysical Chemistry, Göttingen).

Finally, the interactions of hVDAC1 with its major transport solute ATP and with $\mathrm{Ca}^{2+}$ are characterized by solution NMR spectroscopy. For this purpose, titrations are performed with nucleotides, $\mathrm{CaCl}_{2}$, and various $\mathrm{Ca}^{2+}$ analogues described in the literature. 


\section{Materials and Methods}

\subsection{Materials}

\subsubsection{Chemical reagents}

ATP (disodium salt), GTP (lithium salt), UTP (trisodium salt), 2-acrylamido-2-methyl1-propanesulfonic acid (AMPS), (3-acrylamidopropyl)-trimethylammonium chloride (APTMAC) and N,N'-methylenebisacrylamide were purchased from Sigma-Aldrich. A $40 \%$ solution of acrylamide and $\mathrm{N}, \mathrm{N}^{\prime}$-methylenebisacrylamide in a 19:1 ratio and ruthenium red (RuR) were from Applichem. 2'-deoxyguanylyl(3' $\rightarrow$ 5')-2'deoxyguanosine $(\mathrm{dGpG})$ was purchased from Rasajan inc., Pf1 phages from ASLA biotech, lauryl-dimethylamine-N-oxide (LDAO) from Fluka, N,N'dicyclohexylcarbodiimide (DCCD) from Calbiochem-Novabiochem, and (1-oxy2,2,5,5-tetramethyl-3-pyrroline-3-methyl)-methanethiosulfonate (MTSL) from Toronto Research Chemicals. ${ }^{13} \mathrm{C}$-D-glucose $\left(\mathrm{U}_{-}{ }^{13} \mathrm{C} 6,99 \%\right.$ ), deuterated ${ }^{13} \mathrm{C}$-D-glucose (U- ${ }^{13} \mathrm{C} 6$, 99\%; 1,2,3,4,5,6,6-D7, 97-98\%) and ${ }^{15} \mathrm{NH}_{4} \mathrm{Cl}\left({ }^{15} \mathrm{~N}, 99 \%\right)$ were from Cambridge Isotope Laboratories, Sigma-Aldrich, or Cortecnet.

\subsubsection{Bacterial strains}

- Escherichia coli strain M15 [pRep4] (Qiagen, Hilden; phenotype: E. coli K12 $\mathrm{NaI}^{\mathrm{S}} \mathrm{Str}^{\mathrm{S}} \mathrm{Rif}^{\mathrm{S}} \mathrm{Thi}^{-} \mathrm{Lac}^{-} \mathrm{Ara}^{+} \mathrm{Gal}^{+} \mathrm{Mtl}^{-} \mathrm{F}^{-} \mathrm{RecA}^{+} \mathrm{Uvr}^{+} \mathrm{Lon}^{+}$; carries pREP4 (lacI encoding repressor plasmid)). 


\subsubsection{Growth media}

Table 1. M9 minimal medium (standard/deuterated).

\begin{tabular}{|c|c|c|}
\hline Medium & Substance & Amount \\
\hline \multirow[t]{8}{*}{ M9 minimal medium } & M9 salt $(5 x)^{*}$ & $200 \mathrm{ml}$ \\
\hline & Trace elements ${ }^{\#}$ & $10 \mathrm{ml}$ \\
\hline & Thiamine $\mathrm{HCl}(5 \mathrm{mg} / \mathrm{ml})$ & $6 \mathrm{ml}$ \\
\hline & $\mathrm{CaCl}_{2}(2 \mathrm{M})$ & $0.05 \mathrm{ml}$ \\
\hline & $\mathrm{MgSO}_{4}(1 \mathrm{M})$ & $2 \mathrm{ml}$ \\
\hline & $\begin{array}{l}\text { D-Glucose, }{ }^{13} \mathrm{C} \text {-D-glucose or } \\
\text { deuterated }{ }^{13} \mathrm{C} \text {-D-glucose }(20 \% \mathrm{w} / \mathrm{v})\end{array}$ & $20 \mathrm{ml}$ \\
\hline & $\mathrm{NH}_{4} \mathrm{Cl}$ or ${ }^{15} \mathrm{NH}_{4} \mathrm{Cl}(0.25 \mathrm{~g} / \mathrm{ml})$ & $4 \mathrm{ml}$ \\
\hline & $\mathrm{H}_{2} \mathrm{O}$, deionized or $\mathrm{D}_{2} \mathrm{O}$ & $758 \mathrm{ml}$ \\
\hline \multirow[t]{4}{*}{ *) M9 salt (5x) } & $\mathrm{Na}_{2} \mathrm{HPO}_{4}$ & $33.9 \mathrm{~g}$ \\
\hline & $\mathrm{KH}_{2} \mathrm{PO}_{4}$ & $15 \mathrm{~g}$ \\
\hline & $\mathrm{NaCl}$ & $2.5 \mathrm{~g}$ \\
\hline & $\mathrm{H}_{2} \mathrm{O}$, deionized or $\mathrm{D}_{2} \mathrm{O}$ & ad $1,000 \mathrm{ml}$ \\
\hline \multirow[t]{9}{*}{ \#) Trace elements } & $\mathrm{FeSO}_{4} \times 7 \mathrm{H}_{2} \mathrm{O}$ & $0.6 \mathrm{~g}$ \\
\hline & $\mathrm{MnCl}_{2} \times 4 \mathrm{H}_{2} \mathrm{O}$ & $0.115 \mathrm{~g}$ \\
\hline & $\mathrm{CoCl}_{2} \times 6 \mathrm{H}_{2} \mathrm{O}$ & $0.08 \mathrm{~g}$ \\
\hline & $\mathrm{ZnSO}_{4} \times 7 \mathrm{H}_{2} \mathrm{O}$ & $0.07 \mathrm{~g}$ \\
\hline & $\mathrm{CuCl}_{2} \times 2 \mathrm{H}_{2} \mathrm{O}$ & $0.03 \mathrm{~g}$ \\
\hline & $\mathrm{H}_{3} \mathrm{BO}_{3}$ & $0.002 \mathrm{~g}$ \\
\hline & $\left(\mathrm{NH}_{4}\right)_{6} \mathrm{Mo}_{7} \mathrm{O}_{24} \times 4 \mathrm{H}_{2} \mathrm{O}$ & $0.025 \mathrm{~g}$ \\
\hline & EDTA & $0.5 \mathrm{~g}$ \\
\hline & $\mathrm{H}_{2} \mathrm{O}$, deionized or $\mathrm{D}_{2} \mathrm{O}$ & ad $100 \mathrm{ml}$ \\
\hline
\end{tabular}

\subsection{Methods}

\subsubsection{Sample preparation of hVDAC1}

Cloning, expression, and purification of $w t$ and mutant hVDAC1 with a C-terminal His $_{6}$-tag were performed by Stefan Becker and Karin Giller in the Department of NMRbased Structural Biology, Max Planck Institute for Biophysical Chemistry in Göttingen. The preparation of hVDAC1 was done mostly as described (Engelhardt et al. 2007). Detailed protocols can be found in the doctoral theses of Thomas Meins (Meins 2007) and Monika Bayrhuber (Bayrhuber 2007), from which parts of the following sections are reproduced. 


\subsubsection{Cloning of hVDAC1}

The plasmid containing wild type (wt) hVDAC1, cloned into the pDS56/RBSII-6xHis vector via the BamH1 and BglII restriction sites as described (Engelhardt et al. 2007), was kindly provided by Thomas Meins (Max Planck Institute for Biochemistry, Martinsried). The PCR construct of $w t$ hVDAC1 was amplified from this vector and ligated with the NcoI and BglII restriction sites in the pQE60 vector (Qiagen).

\subsubsection{Site directed mutagenesis}

Point mutations into the cloned hVDAC1 coding sequence were introduced with the QuikChange ${ }^{\circledR}$ site-directed mutagenesis kit (Stratagene), which was used essentially as recommended by Stratagene. hVDAC1 mutants, vectors, and primers used in this thesis are listed in Table 2. Successful mutagenesis was verified by DNA sequence analysis.

Table 2. Oligonucleotide primers for the mutagenesis of $\mathrm{hVDAC1}-\mathrm{His}_{6}$.

\begin{tabular}{|c|c|c|c|}
\hline Mutation & Host & Vector* & Primers (forward and reversed) \\
\hline $\mathrm{R} 15 \mathrm{~A}$ & $w t$ & b) & $\begin{array}{l}5^{\prime} \text {-GATCTTGGCAAATCTGCCGCGGATGTCTTCACCAAG-3' } \\
5^{\prime} \text {-CTTGGTGAAGACATCCGCGGCAGATTTGCCAAGATC-3' }\end{array}$ \\
\hline E73V & $w t$ & a) & $\begin{array}{l}5^{\prime} \text {-CGGCCTGACGTTTACAGTGAAATGGAATACCGAC-3' } \\
5^{\prime} \text {-GTCGGTATTCCATTTCACTGTAAACGTCAGGCCG-3' }\end{array}$ \\
\hline E73V & $w t$ & b) & $"$ \\
\hline $\mathrm{C} 127 \mathrm{~S}$ & $w t$ & b) & $\begin{array}{l}5^{\prime}- \\
\text { GAGCACATTAACCTGGGCTCCGACATGGATTTCGACATTG- } \\
3^{\prime} \\
5^{\prime}- \\
\text { CAATGTCGAAATCCATGTCGGAGCCCAGGTTAATGTGCTC- } \\
3^{\prime}\end{array}$ \\
\hline $\mathrm{C} 232 \mathrm{~S}$ & $w t$ & a) & $\begin{array}{l}\text { 5' -GATTGACCCTGACGCCTCCTTCTCGGCTAAAGTG-3' } \\
\text { 5' -CACTTTAGCCGAGAAGGAGGCGTCAGGGTCAATC-3' }\end{array}$ \\
\hline $\mathrm{C} 127 \mathrm{~S} / \mathrm{C} 232 \mathrm{~S}$ & $\mathrm{C} 127 \mathrm{~S}$ & b) & $\begin{array}{l}\text { 5'-GATTGACCCTGACGCCTCCTTCTCGGCTAAAGTG-3' } \\
\text { 5' -CACTTTAGCCGAGAAGGAGGCGTCAGGGTCAATC-3' }\end{array}$ \\
\hline T19C/C127S/C232S & $\mathrm{C} 127 \mathrm{~S} / \mathrm{C} 232 \mathrm{~S}$ & b) & $\begin{array}{l}5^{\prime} \text {-CTGCCAGGGATGTCTTCTGCAAGGGCTATGGATTTG-3' } \\
5^{\prime} \text {-CAAATCCATAGCCCTTGCAGAAGACATCCCTGGCAG-3' }\end{array}$ \\
\hline $\mathrm{S} 43 \mathrm{C} / \mathrm{C} 127 \mathrm{~S} / \mathrm{C} 232 \mathrm{~S}$ & $\mathrm{C} 127 \mathrm{~S} / \mathrm{C} 232 \mathrm{~S}$ & a) & $\begin{array}{l}\text { 5'-GAGAATGGATTGGAATTTACATGCTCAGGCTCAGCCAAC } \\
\text { ACTGAG-3' } \\
5^{\prime}-\text {-CTCAGTGTTGGCTGAGCCTGAGCATGTAAATTCCAATCC } \\
\text { ATTCTC-3' }\end{array}$ \\
\hline $\mathrm{T} 60 \mathrm{C} / \mathrm{C} 127 \mathrm{~S} / \mathrm{C} 232 \mathrm{~S}$ & $\mathrm{C} 127 \mathrm{~S} / \mathrm{C} 232 \mathrm{~S}$ & a) & $\begin{array}{l}\text { 5' -GTGACGGGCAGTCTGGAATGCAAGTACAGATGGACTGAG } \\
\text { TAC-3' } \\
5^{\prime}-\text { GTACTCAGTCCATCTGTACTTGCATTCCAGACTGCCCGT } \\
\text { CAC-3' }\end{array}$ \\
\hline $\mathrm{V} 87 \mathrm{C} / \mathrm{C} 127 \mathrm{~S} / \mathrm{C} 232 \mathrm{~S}$ & $\mathrm{C} 127 \mathrm{~S} / \mathrm{C} 232 \mathrm{~S}$ & a) & $\begin{array}{l}\text { 5'-CACTAGGCACCGAGATTACTTGCGAAGATCAGCTTGCAC } \\
\text { GTG-3' } \\
5^{\prime}-\text { CACGTGCAAGCTGATCTTCGCAAGTAATCTCGGTGCCTA } \\
\text { GTG-3' }\end{array}$ \\
\hline $\mathrm{C} 127 \mathrm{~S} / \mathrm{C} 232 \mathrm{~S} / \mathrm{L} 277 \mathrm{C}$ & $\mathrm{C} 127 \mathrm{~S} / \mathrm{C} 232 \mathrm{~S}$ & a) & $\begin{array}{l}\text { 5'-GGTGGCCACAAGCTTGGTTGCGGACTGGAATTTCAAGCA } \\
\text { AGATC-3' } \\
5^{\prime}-\text { GATCTTGCTTGAAATTCCAGTCCGCAACCAAGCTTGTGG } \\
\text { CCACC-3' }\end{array}$ \\
\hline
\end{tabular}

* The vector was either a) pDS56/RBSII or b) pQE60 


\subsubsection{Expression of ${ }^{2} \mathrm{H},{ }^{13} \mathrm{C}$, and ${ }^{15} \mathrm{~N}$ labelled $\mathrm{hVDAC1}$}

Following transformation into Escherichia coli M15 [pRep4], cells were grown at $37^{\circ} \mathrm{C}$ over night in M9 minimal medium containing $100 \mu \mathrm{g} / \mathrm{ml}$ ampicillin and $25 \mu \mathrm{g} / \mathrm{ml}$ kanamycin. According to the desired labelling $\left({ }^{2} \mathrm{H}(75 \%) /{ }^{13} \mathrm{C} /{ }^{15} \mathrm{~N},{ }^{2} \mathrm{H}(99 \%) /{ }^{13} \mathrm{C} /{ }^{15} \mathrm{~N}\right.$ or ${ }^{2} \mathrm{H}(75 \%) /{ }^{15} \mathrm{~N}$ ), glucose and $\mathrm{NH}_{4} \mathrm{Cl}$ in the M9 minimal medium (Table 1) were replaced with protonated or deuterated ${ }^{13} \mathrm{C}$-D-glucose and/or ${ }^{15} \mathrm{NH}_{4} \mathrm{Cl}$. In order to adapt the culture to $\mathrm{D}_{2} \mathrm{O}$ based media the cells were consecutively inoculated into M9 medium with a $\mathrm{D}_{2} \mathrm{O}$ content of 33,65 and $100 \%$ and incubated at $37^{\circ} \mathrm{C}$ for $24 \mathrm{~h}$ at each step. The fully adapted cell culture was used for inoculation of 1.51 expression cultures with an initial $\mathrm{OD}_{600}$ of $0.1 \mathrm{AU}$. The culture was allowed to grow at $37^{\circ} \mathrm{C}$, until an $\mathrm{OD}_{600}$ of $0.8 \mathrm{AU}$ was reached. Subsequently, hVDAC1-His 6 expression into inclusion bodies was induced with $1 \mathrm{mM}$ IPTG. Cells were harvested $8 \mathrm{~h}$ after induction by centrifugation at $5,000 \mathrm{~g}$ for $30 \mathrm{~min}$, shock frozen in liquid $\mathrm{N}_{2}$ and stored at $-70^{\circ} \mathrm{C}$.

\subsubsection{Refolding and purification of hVDAC1}

Frozen cells were thawed, resuspended in buffer $1(100 \mathrm{mM}$ Tris/HCl pH 7.5; $1 \mathrm{mM}$ EDTA; $5 \mathrm{mM}$ DTT, $100 \mathrm{mM} \mathrm{NaCl}, 0.2 \mathrm{mM}$ phenylmethanesulfonyl fluoride (PMSF)), and incubated with $0.1 \mathrm{mg} / \mathrm{ml}$ lysozyme for $30 \mathrm{~min}$. Following lysozyme treatment, $1 \mathrm{mM} \mathrm{MgCl} 2,0.1 \mathrm{mM} \mathrm{MnCl}_{2}$, and $0.05 \mathrm{mg} / \mathrm{ml}$ DNAse I were added and the cells were lysed by three French press passes. Inclusion bodies were harvested by centrifugation at $5,000 \times g$ for $30 \mathrm{~min}$ at $4^{\circ} \mathrm{C}$ at 20,000 psi. The pellet was resuspended in buffer 1 containing $3 \%(\mathrm{w} / \mathrm{v})$ octyl polyoxyethylene (OPOE), stirred for $2 \mathrm{~h}$ at room temperature and pelleted by centrifugation at $5,000 \times g$ for $30 \mathrm{~min}$ at $4{ }^{\circ} \mathrm{C}$. Finally, the pellet was washed with buffer 1 to remove detergent contaminations. Inclusion bodies were solubilized in buffer $2(100 \mathrm{mM}$ Tris/HCl pH 7.5; $1 \mathrm{mM}$ EDTA; $5 \mathrm{mM}$ DTT, $6 \mathrm{M}$ guanidinium chloride) and insoluble material was removed by centrifugation at $100,000 \times g$ for $45 \mathrm{~min}$. The denatured hVDAC1-His 6 protein containing supernatant was adjusted with buffer 2 to $15 \mathrm{mg} / \mathrm{ml}$ and stored at $-70^{\circ} \mathrm{C}$ until use.

Refolding was performed at $4{ }^{\circ} \mathrm{C}$ by dropwise dilution of solubilized protein in buffer 3 (100 mM Tris/HCl pH 8.0; 1 mM EDTA; 5 mM DTT, 1\% (w/v) LDAO) until a final concentration of $0.6 \mathrm{M}$ guanidinium chloride was reached. The resulting protein 
solution was stirred over night at $4{ }^{\circ} \mathrm{C}$, centrifuged at $100,000 \times \mathrm{g}$ for $45 \mathrm{~min}$, and finally 5-fold diluted with buffer 4 (100 mM phosphate buffer $\mathrm{pH} 7.5)$.

Refolded hVDAC1-His 6 was purified by immolized metal ion affinity chromatography (IMAC). For this purpose, hVDAC1 was bound to a $5 \mathrm{ml} \mathrm{Ni}^{2+}$ Sepharose HP column (GE Healthcare), washed with $100 \mathrm{ml}$ buffer 5 (20 mM phosphate buffer $\mathrm{pH} 7.5 ; 20 \mathrm{mM}$ imidazole; $0.2 \%$ LDAO), and afterwards eluted by $50 \mathrm{ml}$ buffer 6 (20 mM phosphate buffer $\mathrm{pH} 7.5 ; 300 \mathrm{mM}$ imidazole; 0.2\% LDAO). hVDAC1 containing fractions (as judged by SDS-PAGE, see Appendix) were pooled and the protein concentration was measured by UV absorption at $280 \mathrm{~nm}$ wavelength using the theoretical extinction coefficient of $38,390 \mathrm{M}^{-1} 1 \mathrm{~cm}^{-1}$. LDAO was added to the pooled fractions in order to adjust the final concentration of a $0.6 \mathrm{mM} \mathrm{hVDAC1}$ sample to a theoretical LDAO content of $6 \%(\mathrm{w} / \mathrm{v})$, assuming that no detergent is lost during further purification steps. After LDAO addition, the sample was dialysed against NMR buffer (25 mM BisTris/HCl pH 6.8, 0.2\% LDAO) for $4 \mathrm{~h}$ and concentrated with a centrifugal filter device (Amicon Ultra- $30 \mathrm{k}$, Millipore) to a protein concentration of 0.4-1 mM. By comparison with a sample of known LDAO concentration, $1 \mathrm{D}{ }^{1} \mathrm{H}$ NMR spectroscopy revealed typical LDAO concentrations of $2-4 \%$ in the final samples.

Improved sample purification by an additional gel filtration step was applied to some hVDAC1 sample preparations (R15A, C127S/C232S, T19C/C127S/C232S and wt hVDAC1 in pQE60 vector, see Appendix). For this purpose, the pooled hVDAC1 fractions obtained from $\mathrm{Ni}^{2+}$ affinity chromatography were concentrated and applied to a Superdex 200 16/60 column (GE Healthcare) equilibrated with buffer 7 (25 mM BisTris $\mathrm{pH}$ 6.8, $150 \mathrm{mM} \mathrm{NaCl}, 0.2 \%$ LDAO). Eluted hVDAC1 fractions (as judged by SDS-PAGE, see Appendix) were pooled and subjected to dialysis with NMR buffer as described above.

\subsubsection{Chemical modification of hVDAC1 with DCCD}

For modification of glutamic acid residues in hydrophobic surrounding with N,N'dicyclohexylcarbodiimide (DCCD) (Figure 6), the compound was added to $w t$ hVDAC1 from a stock solution in DMF-d7 to a final concentration of $2 \mathrm{mM}$ (final 1\% DMF-d7) and incubated at room temperature for three hours as described (Villinger et al. 2010). 


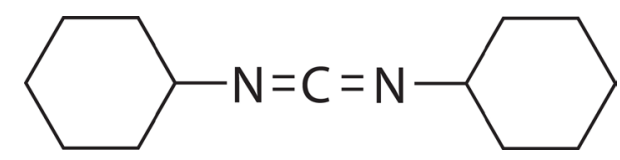

Figure 6. Chemical structure of DCCD.

\subsubsection{Spin labelling of hVDAC1}

T19C/C127S/C232S hVDAC1 was modified with the thiol-specific nitroxide spin label reagent MTSL. $100 \mu \mathrm{g} / \mu \mathrm{l}$ MTSL in cold acetone were added to the protein solution in a three- to five-fold molar excess prior to the refolding step and was incubated for $\sim 1.5 \mathrm{~h}$ at room temperature. Afterwards, refolding and purification was performed as described above.

\subsubsection{Bilayer measurements of hVDAC1 preparations}

Functionality of our hVDAC1 preparation was confirmed by electrophysiological measurements in planar phospholipid bilayer membranes as described (Benz 1994). The measurements were performed using the facilities of Roland Benz (Rudolf Virchow Center, DFG-Research Center for Experimental Biomedicine, University of Würzburg) with the help of Elke Maier. The experimental setup for "black lipid membrane" (BLM) measurements is shown in Figure 7.

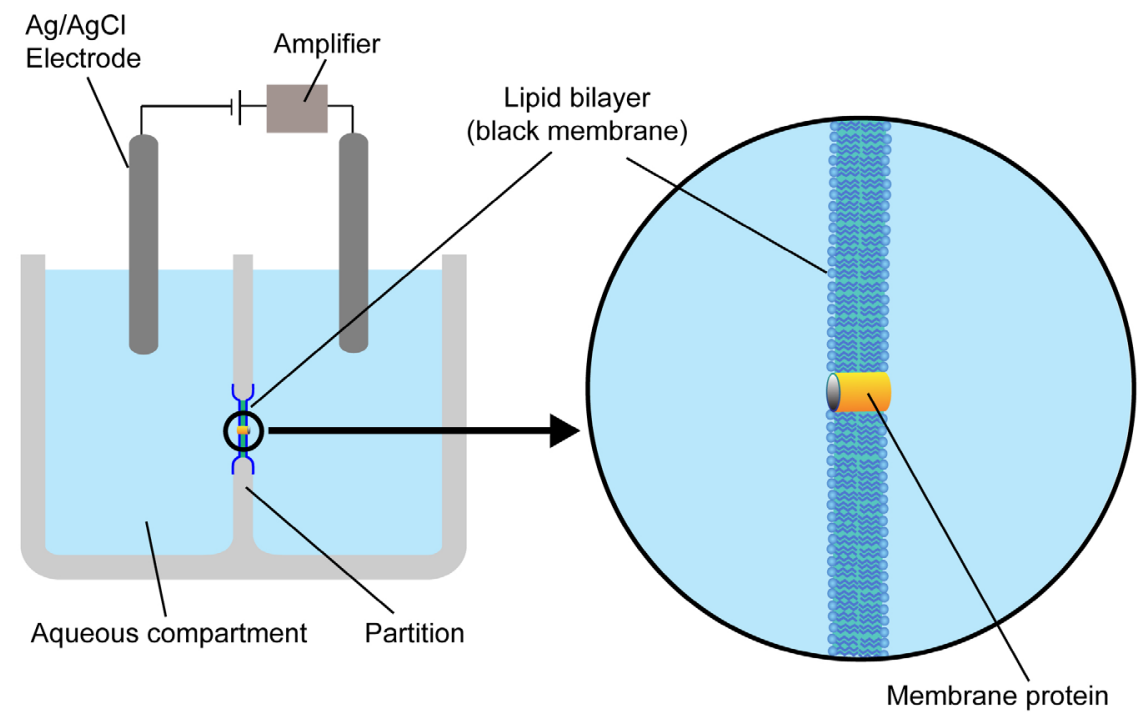

Figure 7. Schematic diagram of BLM measurements. On the left a schematic diagram of the BLM Teflon cuvette with partition is shown. Two $\mathrm{Ag} / \mathrm{AgCl}$ electrodes are used for voltage clamping and current measurements. The right shows a schematic representation of the bilayer lipid membrane containing an ion channel. 
Lipid bilayers of the Mueller-Rudin type (Mueller et al. 1962) were painted by spreading $5 \mu 1$ 1\% diphytanyol-phosphatidylcholine (DiPhPC) in n-decane over the hole (diameter $\sim 0.4-0.5 \mathrm{~mm}$ ) of a Teflon cuvette. The bathing solution contained either $1 \mathrm{M}$ $\mathrm{KCl}$ (unbuffered, $\mathrm{pH} \sim 6$ ) or $0.1 \mathrm{M} \mathrm{KCl}, 20 \mathrm{mM}$ Hepes, $\mathrm{pH}$ 7.2. hVDAC1 in LDAO was diluted with $1 \%(\mathrm{w} / \mathrm{v})$ Genapol to a final concentration of $\sim 5 \mu \mathrm{M}$ and mixed 1:1 with cholesterol powder suspended in $1 \%(\mathrm{w} / \mathrm{v})$ Genapol. Aliquots of hVDAC1 (typically 1-5 $\mu \mathrm{l}$ ) were added to both cuvette chambers at a membrane potential of $+10 \mathrm{mV}$. The insertion of single channels was followed by the increase in current measured with $\mathrm{Ag} / \mathrm{AgCl}$ electrodes and an in-house amplifier with a band width of 33 or $100 \mathrm{~Hz}$. When the membrane ruptured, it was reformed in the same cuvette or prepared freshly in a new cuvette by painting the membrane as described above.

For voltage dependence measurements, the membrane was equilibrated for 1-2 hours until no significant increase in current (i.e. channel insertion) occurred. Then, the steady-state voltage-dependent channel closure was measured at increasing voltage steps of $\pm 10 \mathrm{mV}$ by taking the ratio of the final and initial conductance (current per voltage unit) at each applied voltage step.

\subsubsection{Alignment media preparation}

\subsubsection{Preparation of $d G p G$ liquid crystalline phases}

dGpG was dissolved in $25 \mathrm{mM}$ potassium phosphate $\mathrm{pH} 6.8,44 \mathrm{mM} \mathrm{KCl}, 10 \% \mathrm{D}_{2} \mathrm{O}$ with a final $\mathrm{dGpG}$ concentration of $35 \mathrm{mg} / \mathrm{ml}$, vortexed briefly, and incubated at room temperature over night. LDAO was added from a stock solution of $18 \%$ in $25 \mathrm{mM}$ potassium phosphate, $\mathrm{pH} 6.8$ to a final concentration of $1.8 \%$ (78 mM) LDAO. Alternatively, dGpG was directly dissolved in $25 \mathrm{mM}$ potassium phosphate $\mathrm{pH} 6.8$, $40 \mathrm{mM} \mathrm{KCl}, 10 \% \mathrm{D}_{2} \mathrm{O}$ and $1 \%(44 \mathrm{mM})$ LDAO. The amount of alignment for all samples was determined from the residual ${ }^{2} \mathrm{H}$ quadrupole splitting.

\subsubsection{Preparation of Pf1 phage liquid crystalline phases}

$100 \mu 1$ Pfl bacteriophages (ASLA biotech P-100-P, $50 \mathrm{mg} / \mathrm{ml}$ ) were mixed with $250 \mu 1$ $25 \mathrm{mM}$ Bis-Tris pH 6.8 and vortexed briefly. Phages were pelleted by ultracentrifugation at $60,000 \mathrm{rpm}$ and $4{ }^{\circ} \mathrm{C}$ for $2 \mathrm{~h}$. Pelleted phages were dissolved in 
$250 \mu \mathrm{l}$ of detergent-free buffer (final phage concentration $16.7 \mathrm{mg} / \mathrm{ml}$ ). The phage stock solution was mixed with a stock solution of $22.5 \%$ LDAO in $25 \mathrm{mM}$ BisTris pH 6.8 to obtain samples with varying phage and LDAO concentrations and the amount of alignment was determined from the residual ${ }^{2} \mathrm{H}$ quadrupole splitting obtained from 1D ${ }^{2} \mathrm{H}$ NMR spectra.

\subsubsection{Preparation of charged polyacrylamide gels}

Gels were prepared according to the protocol of T. Cierpicki and co-workers (Cierpicki and Bushweller 2004) with minor modifications. Stock solutions contained 40\% (w/w) of either acrylamide or a charged acrylamide analog, and N-N'-methylenebisacrylamide in a 19:1 ratio (w/w). Positively charged gels were prepared with (3-acrylamidopropyl)trimethylammonium chloride (APTMAC/+M), negatively charged gels with 2acrylamido-2-methyl-1-propanesulfonic acid (AMPS/-S). Stock solutions were mixed to achieve $50 \%$ or $75 \%$ of the charged component and were diluted with $1 \mathrm{M}$ TRIS-HCl $\mathrm{pH} 8.5$ to a final gel concentration of $7.2 \%$. Polymerisation was started by addition of $0.16 \%$ ammoniumperoxide sulfate (APS) and $0.33 \%$ tetramethylethylenediamine (TEMED) and performed over night in plastic tubes (Tygon ${ }^{\circledR}$ tubes, Carl Roth) with an inner diameter of $3.2 \mathrm{~mm}$. In theory, a $\sim 130 \mu \mathrm{l}$ aliquot yields a gel with an optimal length-to-diameter ratio of 5:1 (Cierpicki and Bushweller 2004):

$$
\text { volume }=\pi r^{2} \cdot h=\pi \cdot(1.6 \mathrm{~mm})^{2} \cdot(5 \cdot 3.2) \mathrm{mm}=129 \mu l \text {. }
$$

Here, $300 \mu \mathrm{l}$ aliquots were used for polymerization, obtaining 1-2 gels from each polymerization, depending on the quality of the gel. Washing of gels was performed in deionized water over a period of two days with eight exchanges of water. Washed gels were cut to achieve a 5:1 ratio of length-to-diameter and dried on polypropylene (PP) foil for two days at $37^{\circ} \mathrm{C}$. Dry gels were stored at $4{ }^{\circ} \mathrm{C}$ in sealed Eppendorff tubes until needed.

For preparation of aligned hVDAC1 samples, the dried gel was placed in an NMR microtube (Shigemi inc.), $300 \mu 1$ of NMR sample were added, the plunger was adjusted to $13 \mathrm{~mm}$ height, and the gel was allowed to swell at $4{ }^{\circ} \mathrm{C}$ over night (Figure 8). Using a standard "Shigemi tube" (inner diameter $4.2 \mathrm{~mm}$ ) and a height of $13 \mathrm{~mm}$, the final gel concentration is $\sim 5.2 \%$. 


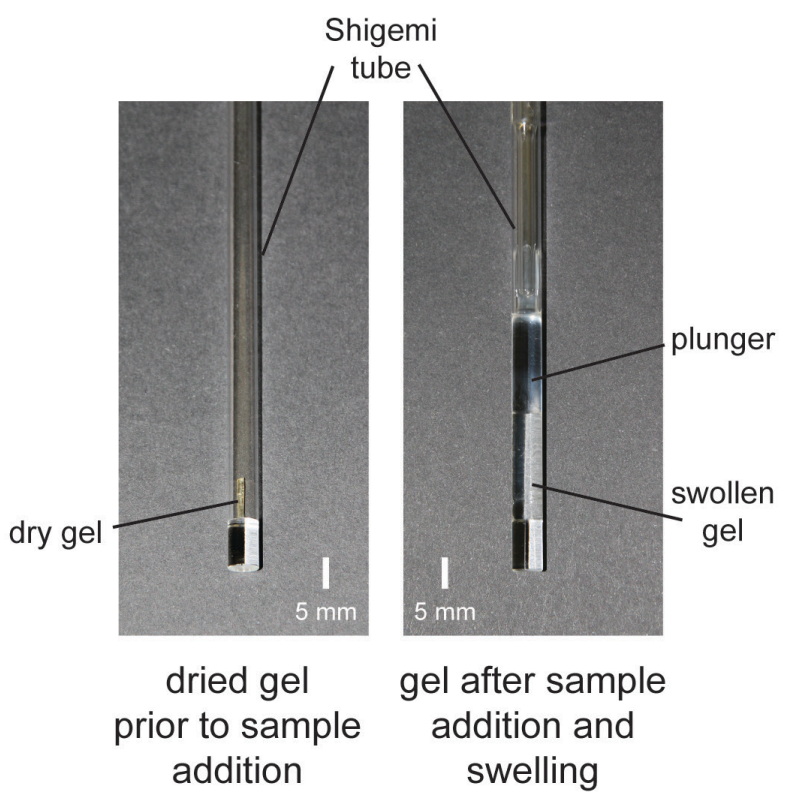

Figure 8. Preparation of polyacrylamide samples for protein alignment. On the left, the dry gel is shown as it is placed into an NMR tube (Shigemi tube). The right side depictures the condition after sample addition and swelling of the gel, with the plunger of the tube inserted and restricting the length of the gel.

When necessary, the alignment (and the gel concentration) was reduced by release of the plunger and equilibrated at $4{ }^{\circ} \mathrm{C}$ over night.

\subsubsection{NMR spectroscopy}

${ }^{2} \mathrm{NMR}$ spectra were recorded on ${ }^{2} \mathrm{H}(75 \%) /{ }^{15} \mathrm{~N},{ }^{2} \mathrm{H}(75 \%) /{ }^{13} \mathrm{C} /{ }^{15} \mathrm{~N}$, or ${ }^{2} \mathrm{H}(99 \%) /{ }^{13} \mathrm{C} /{ }^{15} \mathrm{~N}$ labelled samples containing 0.4-1.0 mM hVDAC1, $25 \mathrm{mM}$ BisTris pH 6.8, 2-4\% (90180) $\mathrm{mM}$ LDAO, and $5-10 \% \mathrm{D}_{2} \mathrm{O}$. All spectra were measured at $37^{\circ} \mathrm{C}$ (unless stated otherwise) on Bruker 600,800 , or $900 \mathrm{MHz}$ spectrometers equipped with cryogenic probes. Spectra were processed using NMRPipe (Delaglio et al. 1995) and analyzed with SPARKY (Goddard and Kneller 2006).

\subsubsection{Assignment improvement}

To further improve the previously obtained backbone resonance assignment (Bayrhuber et al. 2008), a TROSY-based HNCA experiment (Eletsky et al. 2001; Salzmann et al. 1998) was recorded on a sample of ${ }^{2} \mathrm{H}(75 \%) /{ }^{13} \mathrm{C} /{ }^{15} \mathrm{~N} w t$ hVDAC1 with improved purity

${ }^{2}$ Parts of sections 2.2.6, 2.2.7, and 2.2.9 (including subsections) were reproduced from our recent publication (Villinger et al. 2010) and contain identical text passages. 
(see section 2.2.1.4 and Appendix). Together with multiple quantum (MQ) HNCOCA spectra (pulse sequence provided by R. Riek) of conventionally purified $w t$ and E73 hVDAC1 and a TROSY-HNCA of E73 hVDAC1, C $\alpha$ chemical shift connectivity was established. Furthermore, ${ }^{15} \mathrm{~N}$-edited ${ }^{1} \mathrm{H}-{ }^{1} \mathrm{H}$ NOESY-TROSY (Xia et al. 2000) and mixed-time parallel evolution HMQC-NOESY (Ying et al. 2007) spectra of conventionally purified $w t, \mathrm{E} 73 \mathrm{~V}$, and $\mathrm{V} 87 \mathrm{C} / \mathrm{C} 127 \mathrm{~S} / \mathrm{C} 232 \mathrm{~S}$ hVDAC1 were used to extract inter-strand and sequential $\mathrm{H}_{\mathrm{N}}-\mathrm{H}_{\mathrm{N}}$ NOEs. All 3D spectra, parameters, and samples are summarized in Table 3.

Table 3. 3D spectra of $w t$ and mutant hVDAC1 used for assignment improvement.

\begin{tabular}{|c|c|c|c|}
\hline Sample & Spectrum & Parameters $^{\perp}$ & Comments \\
\hline $\begin{array}{l}{ }^{2} \mathrm{H}(75 \%) /{ }^{13} \mathrm{C} /{ }^{15} \mathrm{~N} \\
\mathrm{hVDAC} 1-\mathrm{pQE} 60 ; \\
\text { improved purity }\end{array}$ & TROSY-HNCA & $\begin{array}{l}35 \times 26 \times 512 \mathrm{pts} \\
\mathrm{ns}=72 \\
900 \mathrm{MHz}\end{array}$ & $\begin{array}{l}\text { linear prediction in }{ }^{13} \mathrm{C} \\
\text { and }{ }^{15} \mathrm{~N} \text { dimensions }\end{array}$ \\
\hline \multirow[t]{2}{*}{$\begin{array}{l}{ }^{2} \mathrm{H}(99 \%) /{ }^{13} \mathrm{C} /{ }^{15} \mathrm{~N} \\
\text { hVDAC1- pDS56/RBSII }\end{array}$} & MQ-HNCOCA* & $\begin{array}{l}12 \times 20 \times 512 \mathrm{pts} \\
\mathrm{ns}=268 \\
600 \mathrm{MHz}\end{array}$ & $\begin{array}{l}\text { linear prediction in }{ }^{13} \mathrm{C} \\
\text { and }{ }^{15} \mathrm{~N} \text { dimensions }\end{array}$ \\
\hline & ${ }^{15} \mathrm{~N}-\mathrm{NOESY}$-TROSY* & $\begin{array}{l}94 \times 20 \times 512 \mathrm{pts} ; \\
\mathrm{ns}=32 ; \mathrm{mt}=160 \mathrm{~ms} \\
800 \mathrm{MHz}\end{array}$ & \\
\hline \multirow{4}{*}{$\begin{array}{l}{ }^{2} \mathrm{H}(75 \%) /{ }^{15} \mathrm{~N}- \\
\mathrm{V} 87 \mathrm{C} / \mathrm{C} 127 \mathrm{~S} / \mathrm{C} 232 \mathrm{~S} \\
\mathrm{hVDAC} 1-\mathrm{pDS} 56 / \mathrm{RBSII} \\
{ }^{2} \mathrm{H}(75 \%) /{ }^{13} \mathrm{C} /{ }^{15} \mathrm{~N}-\mathrm{E} 73 \mathrm{~V} \\
\mathrm{hVDAC} 1-\mathrm{pDS} 56 / \mathrm{RBSII}\end{array}$} & ${ }^{15} \mathrm{~N}-\mathrm{NOESY}$-HMQC* & $\begin{array}{l}85 \times 25 \times 512 \mathrm{pts} \\
\mathrm{ns}=26 ; \mathrm{mt}= \\
240 \mathrm{~ms} ; 800 \mathrm{MHz}\end{array}$ & \\
\hline & TROSY-HNCA & $\begin{array}{l}35 \times 26 \times 512 \mathrm{pts} \\
\mathrm{ns}=72 \\
900 \mathrm{MHz}\end{array}$ & $\begin{array}{l}\text { linear prediction in }{ }^{13} \mathrm{C} \\
\text { and }{ }^{15} \mathrm{~N} \text { dimensions }\end{array}$ \\
\hline & MQ-HNCOCA & $\begin{array}{l}12 \times 20 \times 512 \mathrm{pts} \\
\mathrm{ns}=216 \\
600 \mathrm{MHz}\end{array}$ & $\begin{array}{l}\text { linear prediction in }{ }^{13} \mathrm{C} \\
\text { and }{ }^{15} \mathrm{~N} \text { dimensions }\end{array}$ \\
\hline & ${ }^{15} \mathrm{~N}-\mathrm{NOESY}-\mathrm{HMQC}$ & $\begin{array}{l}85 \times 25 \times 512 \mathrm{pts} \\
\mathrm{ns}=26 ; \mathrm{mt}= \\
240 \mathrm{~ms} ; 800 \mathrm{MHz}\end{array}$ & \\
\hline
\end{tabular}

* recorded by Monika Bayrhuber;

${ }^{\perp}$ spectroscopic parameters given: number of complex points(pts) in $t 1, t 2$ and t 3 time domain; number of scans (ns); mixing time (mt); field strength for ${ }^{1} \mathrm{H}$

Furthermore, 2D ${ }^{1} \mathrm{H},{ }^{15} \mathrm{~N}$-TROSY spectra (Zhu et al. 1999) of ${ }^{2} \mathrm{H}(75 \%) /{ }^{15} \mathrm{~N}$ hVDAC1 were measured at varying temperatures $\left(37^{\circ} \mathrm{C}, 32{ }^{\circ} \mathrm{C}, 27^{\circ} \mathrm{C}\right.$, and $\left.22^{\circ} \mathrm{C}\right)$ in order to discriminate overlapping peaks. In addition, $\mathrm{N}_{\mathrm{H}}$ and $\mathrm{C} \alpha$ chemical shifts assigned by solid state NMR in the N-terminal helix (Schneider et al. 2010) were taken into consideration. 


\subsubsection{Determination of secondary chemical shifts}

NMR spectra were referenced with NMRPipe (Delaglio et al. 1995) and shifted by additional $+0.3 \mathrm{ppm}$ in the ${ }^{13} \mathrm{C}$ dimension, to improve the match with solid state NMR shifts. C $\alpha$ and C' secondary chemical shifts were obtained by substracting random coil chemical shifts for charged (Wishart et al. 1995) and uncharged (Schwarzinger et al. 2001) residues, respectively, from the measured chemical shift values.

\subsubsection{Solvent exchange}

In order to identify solvent protected amide protons, ${ }^{2} \mathrm{H}(75 \%) /{ }^{15} \mathrm{~N}$-labeled $w t$ and ${ }^{2} \mathrm{H}(75 \%) /{ }^{13} \mathrm{C} /{ }^{15} \mathrm{~N}$-labeled E73V hVDAC1 were lyophilised and dissolved in $\mathrm{D}_{2} \mathrm{O}$. Subsequently, series of $2 \mathrm{D}{ }^{1} \mathrm{H},{ }^{15} \mathrm{~N}-\mathrm{TROSY}$ spectra (Zhu et al. 1999) were recorded and the resonance intensity ratios $I_{D_{2} \mathrm{O}} / I_{\mathrm{H}_{2} \mathrm{O}}$ evaluated. Errors in intensity ratios were calculated by error propagation using the noise of the spectra estimated by SPARKY.

\subsubsection{Temperature coefficients}

Temperature coefficients (in $\mathrm{ppb} / \mathrm{K}$ ) were obtained from the slope of plots of amide proton or amide nitrogen peak positions (obtained from ${ }^{1} \mathrm{H},{ }^{15} \mathrm{~N}-\mathrm{TROSY}$ spectra at $37^{\circ} \mathrm{C}, 32{ }^{\circ} \mathrm{C}, 27^{\circ} \mathrm{C}$, and $22^{\circ} \mathrm{C}$ ) versus temperature by linear regression analysis in Excel (Microsoft). Errors in temperature coefficients are given as standard errors from the linear regression; errors in average values as standard errors of the mean. Errors in the difference of temperature coefficients were calculated by error propagation using the standard errors.

\subsubsection{Steady state heteronuclear $\left\{{ }^{1} H\right\},{ }^{15} \mathrm{~N}$-nuclear Overhauser effects (NOEs)}

Steady state heteronuclear $\left\{{ }^{1} \mathrm{H}\right\},{ }^{15} \mathrm{~N}-\mathrm{NOE}$ were measured employing the TROSY scheme (Zhu et al. 2000). Heteronuclear NOE values are reported as the ratio of peak intensities in paired interleaved spectra collected with and without initial proton saturation period $(4 \mathrm{~s})$ during the 5-s-recycle delay. The two experiments were measured with $101 \times 512$ complex points (in t1 and t2) and 120 scans. A three residue window averaging was applied to the values, and errors were calculated as the standard error of the mean. 


\subsubsection{Peak intensities as indicators for chemical exchange}

Peak intensities for $w t$ hVDAC1 were collected in triplicate and averaged, using two ${ }^{1} \mathrm{H},{ }^{15} \mathrm{~N}$-TROSY spectra and one reference spectrum of a heteronuclear NOE experiment without saturation. Peak intensities for R15A and E73 hVDAC1 were determined from a single ${ }^{1} \mathrm{H},{ }^{15} \mathrm{~N}$-TROSY spectrum. To reduce broadening due to exchange of labile amide protons, water flip-back pulse sequences were used (Zhu et al. 1999). All ${ }^{1} \mathrm{H},{ }^{15} \mathrm{~N}-$ TROSY spectra were measured with $128 \times 512$ complex points (in $\mathrm{t} 1$ and $\mathrm{t} 2$ ) and 24 to 64 scans. Peak intensities were scaled arbitrarily using the number of scans and protein concentration as a rough estimate. Errors in average peak intensities were calculated as standard error of the mean.

\subsubsection{Chemical exchange rates $\left(R_{e x}\right)$}

Chemical exchange rates $\left(\mathrm{R}_{\mathrm{ex}}\right)$ were determined from a set of three TROSY-based Hahn echo experiments optimized for large proteins (Wang et al. 2003) (Figure 9; pulse sequences and acquisition parameters are given in the Appendix).

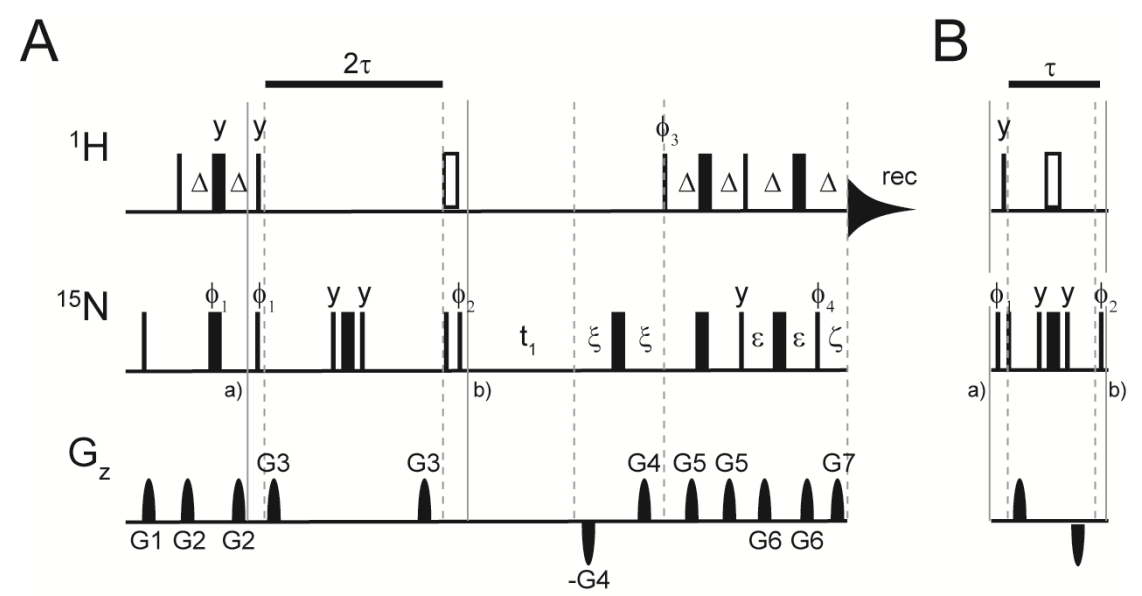

Figure 9. Hahn-echo pulse sequences for detection of chemical exchange in large proteins. Modified from (Wang et al. 2003). The sequence shown in (A) detects relaxation of the narrow doublet component during the Hahn echo period $2 \tau$ when the proton composite pulse element (open bar) is $\left(90^{\circ} \times 0^{\circ} 90^{\circ}-y 90^{\circ}\right.$ ) and detects relaxation of the broad doublet component when this element is $\left(90^{\circ}{ }_{x} 90^{\circ}{ }_{y} 90^{\circ}{ }_{y} 90^{\circ}\right)$. Relaxation of longitudinal two-spin order is detected if the sequence between points a) and b) in (A) is replaced with (B); the proton composite pulse is $\left(90^{\circ} x 0^{\circ} y 0^{\circ} y 0^{\circ} x\right)$. Composite pulses are applied at the center of amide ${ }^{1} \mathrm{H}$. Narrow and wide solid bars are $90^{\circ}$ and $180^{\circ}$ pulses, respectively. All pulses have $x$ phase unless otherwise stated. Phase cycles are $\phi_{1}=\mathrm{x},-\mathrm{x} ; \phi_{2}=\mathrm{x}, \mathrm{x},-\mathrm{x},-\mathrm{x} ; \phi_{3}=\mathrm{y}$; and $\phi_{4}=$ $\mathrm{x}$; receiver phase $=-x, x, x,-x$. Gradients $\mathrm{G} 4$ and $\mathrm{G} 7$ are used for coherence selection; other gradients are for artifact suppression. Echo-antiecho quadrature detection is achieved by inverting $\phi_{3}, \phi_{4}$, and the sign of gradient G4; axial peaks are shifted by inverting $\phi_{1}$ and the receiver phase. Other delays are $\Delta=2.7 \mathrm{~ms}$, $\xi=950, \varepsilon=2.675 \mathrm{~ms}, \zeta=250 \mu \mathrm{s}$. All pulse phases are given in Bruker notation. 
The three experiments were used to obtain peak intensities $I^{N_{x} H_{\alpha}^{N}}(2 \tau)$, $I^{N_{x} H_{\beta}^{N}}(2 \tau)$, and $I^{2 N_{z} H_{z}^{N}}(\tau)$ from a single value of $\tau$, resulting from the magnetization decays of the narrow $(\alpha)$ and broad $(\beta)$ components of the ${ }^{15} \mathrm{~N}$ doublet and the magnetization decay of the longitudinal two-spin order $\left(2 N_{z} H_{z}^{N}\right)$ :

$$
\begin{gathered}
I^{N_{x} H_{\alpha}^{N}}(2 \tau)=I(0) \exp \left[-2 \tau R_{2}^{N_{x} H_{\alpha}^{N}}\right], \\
I^{N_{x} H_{\beta}^{N}}(2 \tau)=I(0) \exp \left[-2 \tau R_{2}^{N_{x} H_{\beta}^{N}}\right], \\
I^{2 H_{z} N_{z}}(\tau)=I(0) \exp \left[-\tau R_{1}^{2 H_{z} N_{z}}\right] .
\end{gathered}
$$

Here, $R_{2}^{N_{x} H_{\alpha}^{N}}$ is the transverse relaxation rate constant for the ${ }^{15} \mathrm{~N}$ spin with an attached proton in the $\alpha$ state, $R_{2}^{N_{x} H_{\beta}^{N}}$ is the transverse relaxation rate constant for the ${ }^{15} \mathrm{~N}$ spin with an attached proton in the $\beta$ state, and $R_{1}^{2 H_{z} N_{z}} \approx R_{1}^{H}+R_{1}^{N}$ is the relaxation rate constant for longitudinal two-spin order, and $R_{1}^{H}$ and $R_{1}^{N}$ are the longitudinal relaxation rates of the ${ }^{1} \mathrm{H}$ and ${ }^{15} \mathrm{~N}$ spin, respectively. By rearranging the intensity ratios from equations (2), (3), and (4) one yields further:

$$
\begin{gathered}
I^{N_{x} H_{\beta}^{N}}(2 \tau) / I^{N_{x} H_{\alpha}^{N}}(2 \tau)=\exp \left[-4 \tau \eta_{x y}\right], \\
I^{N_{x} H_{\alpha}^{N}}(2 \tau) / I^{2 H_{z} N_{z}}(\tau)=\exp \left[-2 \tau\left(R_{2}^{N_{x} H_{\alpha}^{N}}-R_{1}^{2 H_{z} N_{z}} / 2\right)\right]
\end{gathered}
$$

Here, $\eta_{x y}$ is the rate constant for the ${ }^{1} \mathrm{H}^{-}{ }^{15} \mathrm{~N}$ dipole-dipole $/{ }^{15} \mathrm{~N}$ chemical shift anisotropy relaxation interference for transverse magnetization. $\mathrm{R}_{\mathrm{ex}}$ for proteins larger than $30 \mathrm{kDa}$ can then be determined as (Wang et al. 2003):

$$
R_{e x}=R_{2}^{N_{x} H_{\alpha}^{N}}-R_{1}^{2 H_{z} N_{z}} / 2-\eta_{x y}(\kappa-1) .
$$

The scaling factor $\kappa$ can be calculated theoretically or determined experimentally from non-exchanging residues (see below).

The three spectra were measured interleaved with $122 \times 512$ complex points and 240 transients for $w t \mathrm{hVDAC} 1$ and $74 \times 512$ complex points and 320 transients for $\mathrm{E} 73 \mathrm{~V}$ $\mathrm{hVDAC1}$. The shortest possible relaxation delay of $2 \tau=1 / \mathrm{J}=10.87 \mathrm{~ms}$ was used, 
corresponding to $I^{N_{x} H_{\beta}^{N}} / I^{N_{x} H_{\alpha}^{N}}$ of $\sim 0.2$ for most residues. $\kappa$ was estimated by the mean of $1+\left(R_{2}^{N_{x} H_{\alpha}^{N}}-\mathrm{R}_{1}{ }^{2 \mathrm{HzNz}} / 2\right) / \eta_{\mathrm{xy}}$ using all residues in $\beta$-strands that were apparently not exchanging in $w t$ hVDAC1 ( $\beta 8-\beta 11, \beta 14-\beta 15$ and $\beta 18)$, resulting in a value of 1.15 (1.17) for $w t$ (E73V) hVDAC1. For non-exchanging residues $\left(-10<\mathrm{R}_{\mathrm{ex}}<+10\right)$, $\mathrm{R}_{\mathrm{ex}}$ is distributed around $0.75 \mathrm{~s}^{-1}\left(0.65 \mathrm{~s}^{-1}\right)$ with a standard deviation of $5 \mathrm{~s}^{-1}$ due to variation in magnitude and orientation of the ${ }^{15} \mathrm{~N}$ CSA tensor (Fushman et al. 1998). Very weak peaks that resulted in unusually negative values for $\mathrm{R}_{\mathrm{ex}}$ were excluded from the analysis: K224-I227, A231, and F233 for wt hVDAC1; Y225-I227, A231, and F233 for E73V hVDAC1. Errors in $\mathrm{R}_{\mathrm{ex}}$ were calculated by error propagation using the standard error of the mean as uncertainty in $\kappa$ and the estimated noise of the spectra as uncertainties in $I^{N_{x} H_{\alpha}^{N}}(2 \tau), I^{N_{x} H_{\beta}^{N}}(2 \tau)$, and $I^{2 N_{z} H_{z}^{N}}(\tau)$.

\subsubsection{Estimation of $T 2$ and the global rotational correlation time}

A ${ }^{1} \mathrm{H} 1 \mathrm{D}$ (1-1) echo experiment (Sklenáŕ and Bax 1987) with delays of 0.11, 1, 1.5, and $3 \mathrm{~ms}$ was recorded on $w t$ and $\mathrm{T} 19 \mathrm{C} / \mathrm{C} 127 \mathrm{~S} / \mathrm{C} 232 \mathrm{~S}$ hVDAC1. Transverse relaxation times $\left(\mathrm{T}_{2}\right)$ were estimated from two values or from a single-exponential curve fit. The rotational correlation time $\tau_{\mathrm{C}}$ was estimated as:

$$
\tau_{C} \approx \frac{1}{5 \cdot T_{2}}
$$

Alternatively, $\tau_{\mathrm{C}}$ of the protein/micelle complex was estimated by recording a $\left[{ }^{15} \mathrm{~N},{ }^{1} \mathrm{H}\right]$-TRACT experiment (Lee et al. 2006). Here, the integral over the wellstructured part of the amide proton region $(8.5-10 \mathrm{ppm})$ or a strong signal in this region $\left({ }^{1} \mathrm{H}\right.$ chemical shift of $\left.9.09 \mathrm{ppm}\right)$ were used to determine the relaxation rates $R_{2}^{N_{x} H_{\alpha}^{N}}$ and $R_{2}^{N_{x} H_{\beta}^{N}}$ by exponential curve fitting using the program Origin (Origin Lab, Northampton, MA). From these rates $\tau_{\mathrm{C}}$ was calculated as described (Lee et al. 2006). Errors in $\tau_{\mathrm{C}}$ were estimated by calculating its minimum and maximum value using the standard errors in relaxation rates determined from the exponential curve fit.

A comparison with OmpX reconstituted in DHPC micelles allows estimation of the expected monomeric molecular weight and correlation time as follows (Bayrhuber et al. 2008). DHPC with two lipid chains and a molecular weight of 453 Da corresponds 
to two LDAO molecules with only one lipid chain of molecular weight of $229 \mathrm{Da}$. OmpX has a molecular weight of $16 \mathrm{kD}$ and $8 \beta$-strands. Its correlation time is $21-24 \mathrm{~ns}$ in DHPC micelles corresponding to an overall molecular weight of $\sim 60 \mathrm{kD}$ (Lee et al. 2006). The circumference of VDAC with $19 \beta$-strands is $19 / 8$ times that of OmpX, resulting in a theoretical molecular weight of $\sim 143 \mathrm{kDa}$ and a corresponding rotational correlation time of 50-57 ns. Considering the elevated temperature used for hVDAC1 measurements $\left(37^{\circ} \mathrm{C}\right.$ compared to $30^{\circ} \mathrm{C}$ for OmpX (Lee et al. 2006)), a slightly lower rotational correlation time is expected for monomeric hVDAC1.

\subsubsection{Determination of residual ${ }^{2}$ H quadrupole splitting}

Samples in various alignment media were supplied with $5-10 \% \mathrm{D}_{2} \mathrm{O}$ and $1 \mathrm{D}{ }^{2} \mathrm{H} \mathrm{NMR}$ spectra were recorded on Bruker $400 \mathrm{MHz}$ spectrometers equipped with a room temperature probe.

\subsubsection{RDC measurements and evaluation with PALES}

For extraction of amide ${ }^{1} \mathrm{H}_{-}{ }^{15} \mathrm{~N}$ residual dipolar couplings $\left({ }^{1} \mathrm{D}_{\mathrm{NH}}\right)$ a ${ }^{1} \mathrm{H},{ }^{15} \mathrm{~N}-\mathrm{TROSY}$ and a ${ }^{1} \mathrm{H},{ }^{15} \mathrm{~N}-\mathrm{HSQC}$ spectrum were recorded according to (Kontaxis et al. 2000). The water flip-back ${ }^{1} \mathrm{H},{ }^{15} \mathrm{~N}$-TROSY pulse sequence used in all hVDAC1 experiments (pulse sequence in Figure 1c in reference (Zhu et al. 1999)) and a ${ }^{1} \mathrm{H},{ }^{15} \mathrm{~N}-\mathrm{HSQC}$ with sensitivity enhancement and gradient selection (Kay et al. 1992; Palmer III et al. 1991; Schleucher et al. 1994) were recorded in an interleaved fashion with $128 \times 512$ complex points for each spectrum and 108 (TROSY) and 432 (HSQC) transients. From the difference in peak positions in the ${ }^{15} \mathrm{~N}$ dimension and an average ${ }^{1} \mathrm{~J}_{\mathrm{NH}}$ value of $-92 \mathrm{~Hz}$, ${ }^{1} \mathrm{D}_{\mathrm{NH}}$ values were calculated as:

$$
{ }^{1} D_{N H}=2 \cdot\left(v_{N}^{H S Q C}-v_{N}^{T R O S Y}\right)-{ }^{1} J_{N H} \cdot
$$

In addition, an interleaved 2D "RDC-TROSY" (Bhattacharya et al. 2010) was recorded with $\kappa$ values of $0,0.25,0.5$, and 0.8 for aligned samples and $0,0.25,0.5$, 0.75 , and 1.0 for isotropic samples, respectively (see Appendix).

${ }^{1} \mathrm{D}_{\mathrm{NH}}$ values extracted from interleaved TROSY/HSQC spectra were used to calculate the magnitude (normalized to ${ }^{1} \mathrm{D}_{\mathrm{NH}}$ ) and the rhombicity of the alignment tensor using the program PALES (Zweckstetter 2008). Furthermore, RDC values were 
back-calculated from the crystal structure of mVDAC1 (PDB code: 3EMN) using singular value decomposition as implemented in PALES. A comparison of experimental and predicted RDC values was used to describe the quality of the RDCs.

\subsubsection{Interaction studies}

Interaction of hVDAC1 with ATP (disodium salt), MgATP (disodium ATP $+\mathrm{MgCl}_{2}$ excess), GTP (lithium salt), UTP (trisodium salt), $\mathrm{CaCl}_{2}, \mathrm{GdCl}_{3}$, and $\mathrm{RuR}$ was investigated by titration of $w t$ or mutant ${ }^{2} \mathrm{H}(75 \%)-{ }^{15} \mathrm{~N}$ hVDAC1 as indicated in Table 4 with the respective salt from a stock solution up to a 64-fold molar excess with respect to the protein. As a control for salt effects, hVDAC1 was titrated with $50 \mathrm{mM}$ and $100 \mathrm{mM} \mathrm{NaCl}$ (Table 4). To probe for the presence of $\mathrm{Ca}^{2+}$ traces, hVDACl was titrated with $5 \mathrm{mM}$ EGTA, followed by an increasing excess of $\mathrm{CaCl}_{2}$ (Table 4). All mutants displayed similar NMR spectra, with shifted resonances observed only for residues close to the mutation sites. Thus, the mutants were assumed to be functional and to share a similar 3D structure as $w t$ hVDAC1.

Table 4. hVDAC1 samples used for interaction studies.

\begin{tabular}{ll}
\hline Interacting substance / molecule & hVDAC1 samples \\
\hline ATP (disodium salt) & $w t$ \\
MgATP (ATP disodium salt, 10-20 mM MgCl 2 excess) & $w t$ \\
GTP (disodium salt) & T60C-C127S-C232S \\
UTP (lithium salt) & V87C-C127S-C232S \\
$\mathrm{NaCl}$ & T60C-C127S-C232S \\
$\mathrm{RuR}$ & L277C-C127S-C232S \\
$\mathrm{CaCl}_{2}{ }^{*}$ & $w t$ \\
$\mathrm{GdCl}_{3}{ }^{*}$ & $w t$ \\
$\mathrm{EGTA}^{\mathrm{CCaEGTA}}$ & $w t$ \\
\hline
\end{tabular}

* recorded by Monika Bayrhuber

Stock solutions were prepared in $25 \mathrm{mM}$ BisTris pH 6.8 , with $6 \%$ or without LDAO. The $\mathrm{pH}$ of the stock solutions was adjusted with $\mathrm{HCl}$ and $\mathrm{NaOH}$, and the sample $\mathrm{pH}$ was kept constant during all titrations by the addition of $\mathrm{NaOH}$ or $\mathrm{HCl}$, if necessary. The MgATP stock solution (without LDAO) was prepared by mixing the $\mathrm{Na}_{2} \mathrm{ATP}$ stock solution with equimolar amounts of $\mathrm{MgCl}_{2}$. The reference sample for the MgATP titration contained $10 \mathrm{mM} \mathrm{MgCl}_{2}$, such that a $10 \mathrm{mM} \mathrm{Mg}^{2+}$ excess over ATP was maintained at the intial titration steps (1-, 2-, 4-, 16-, and 32-fold MgATP excess). 
For the last step (64-fold molar excess), the $\mathrm{Mg}^{2+}$ excess was increased to $20 \mathrm{mM}$ by further addition of $\mathrm{MgCl}_{2}$. According to calculations (Schoenmakers et al. 1992), at all titration steps $>99 \%$ of the ATP is thus found in complex with $\mathrm{Mg}^{2+}$.

${ }^{1} \mathrm{H},{ }^{15} \mathrm{~N}$-TROSY spectra were recorded in order to follow ${ }^{15} \mathrm{~N}$ and ${ }^{1} \mathrm{H}$ chemical shift changes and variations in peak intensities. Some resonances close to the mutation sites were only ambiguously assigned and excluded from the analysis. Severely overlapping resonances were excluded from the analysis of peak intensities. Normalized weighted average chemical shift differences (CSD), $\Delta_{\mathrm{HN}}$, were calculated as:

$$
\Delta_{H N}=\sqrt{\frac{\left(\Delta \delta_{N} / 5\right)^{2}+\left(\Delta \delta_{H}\right)^{2}}{2}},
$$

where $\Delta \delta_{\mathrm{N}}$ and $\Delta \delta_{\mathrm{H}}$ are the chemical shift differences for the ${ }^{15} \mathrm{~N}$ and ${ }^{1} \mathrm{H}$ dimensions, respectively. Intensity ratios $\left(\mathrm{I}_{\text {solute }} / \mathrm{I}_{\mathrm{ref}}\right)$ were scaled according to resonances that exhibited almost no CSD and similar changes in peak intensities. CSD and intensity changes were mapped onto the crystal structure (PDB code: 3EMN) or the combined NMR- and crystal structure (PDB code: 2JK4) and visualized with Pymol (DeLano 2003). Errors in CSD were estimated by duplicate measurement of a wt hVDAC1 sample with typical protein concentration $(0.5 \mathrm{mM})$ and signal-to-noise ratio and are given as average values (0.014 ppm for ${ }^{15} \mathrm{~N}$ CSD, $0.0028 \mathrm{ppm}$ for the average CSD). Errors in resonance intensity ratios were calculated by error propagation using the estimated noise of individual spectra.

Due to the increased salt concentration, an overall reduction in signal-to-noise was observed during titration steps. For ATP and MgATP titrations, resonance intensities of the spectra upon ligand addition were scaled according to residues that exhibited no shifts and similar intensity changes. Resonances that exhibited shifts but only small intensity changes (less than $20 \%$ in the final titration step) after intensity scaling were classified as resonances in fast exchange. For these resonances a plot of CSD versus ligand concentration was prepared and $\mathrm{K}_{\mathrm{D}}$ values were determined by curve fitting to a single-site binding model (Cavanagh et al. 2007) with the program Origin (OriginLab, Northampton, MA):

$$
\Delta=\Delta_{\max }\left([L]_{T}+[P]_{T}+K_{D}-\left\{\left([L]_{T}+[P]_{T}+K_{D}\right)^{2}-4[L]_{T}[P]_{T}\right\}^{1 / 2}\right) /\left(2[P]_{T}\right) .
$$


Here, $\Delta$ is the CSD, $\Delta_{\max }$ the maximum CSD value at saturation, $[\mathrm{L}]_{\mathrm{T}}$ the total ligand concentration and $[\mathrm{P}]_{\mathrm{T}}$ the total protein concentration. Changes of $[\mathrm{P}]_{\mathrm{T}}$ were kept to a minimum during the titration, and the average protein concentration was used as a fixed value of $[\mathrm{P}]_{\mathrm{T}}$. $\Delta_{\max }$ and $\mathrm{K}_{\mathrm{D}}$ were simultaneously fit. Errors are given as standard errors obtained from the curve fitting.

\subsubsection{Gaussian Network Model Analysis}

Normal modes were calculated for the mVDAC1 crystal structure (Ujwal et al. 2008) using a standard $\mathrm{C} \alpha$-atom based Gaussian Network Model available as the online server oGNM (Yang et al. 2006). A default value of $10 \AA$ was used as a cutoff $\left(\mathrm{r}_{\mathrm{c}}\right)$ for construction of the Kirchhoff matrix using $\mathrm{C} \alpha$-atoms.

\subsubsection{Electrostatic potential calculations}

Electrostatic potential maps of the crystal structure of mVDAC1 (PDB code: 3EMN) were calculated with the program Delphi (Accelrys). 251 grid points were used with a $1.21 \AA$ coarse grid size and $0.5 \AA$ fine grid size. The ionic concentration was set to $0.1 \mathrm{M}$, dielectric constants of the protein and water were set to 4 and 80 , respectively.

\subsubsection{MD simulations and principal component analysis (PCA)}

MD simulations and principal component analysis (PCA) of mVDAC1 in a DMPC lipid bilayer was performed by Bert L. de Groot, Rodolfo Briones, and Ulrich Zachariae from the department of Theoretical and Computational Biophysics, Max Planck Institute for Biophysical Chemistry in Göttingen. MD simulations were performed for 100-150 ns using the crystal structure of mVDAC1 (Ujwal et al. 2008) and modified versions thereof as described in our publication (Villinger et al. 2010), from which the following paragraphs were reproduced.

MD simulations were performed using Gromacs 4.0 (Hess et al. 2008) together with the OPLS (Jorgensen et al. 1996) and amber99sb force-fields (Wang et al. 2004), respectively, for the protein, water, and ions. The initial structure of mVDAC1 was taken from the PDB structure 3EMN (Ujwal et al. 2008). Protonation states of the protein ionizable groups were determined using Whatif (Vriend 1990). mVDAC1 was 
embedded in an equilibrated and fully hydrated DMPC bilayer derived from Berger et al. (Berger et al. 1997) comprising 392 lipid molecules by employing g_membed (Wolf et al. 2010). The surrounding aqueous solution consisted of 13953 water molecules using the TIP4P model (Jorgensen et al. 1983) in the OPLS simulations, and of 12937 water molecules employing the TIP3P model (Jorgensen et al. 1983) in the case of amber99sb. The salt concentration was $0.15 \mathrm{M}$ in all cases, using $\mathrm{K}^{+}$and $\mathrm{Cl}^{-}$ions. In the OPLS simulations, $8 \mathrm{~K}^{+}$and $11 \mathrm{Cl}^{-}$were used to neutralize the system. Hydrogen atoms were treated using the virtual sites approach in the OPLS simulations (Feenstra et al. 1999), allowing for an integration time-step of 4 fs. In the amber simulations, a timestep of 2 fs was used in connection with fully atomistic treatment of hydrogen atoms. The temperature was kept constant by weakly $(\tau=0.1 \mathrm{ps})$ coupling the lipids, protein and solvent separately to a temperature bath of $320 \mathrm{~K}$ using the v-rescale method. The temperature was slightly raised to prevent transitions of DMPC into the gel phase. Likewise, the pressure in the system was kept constant by semi-isotropic coupling to a pressure of 1 bar. Before the start of production runs, equilibration of the system was performed by applying position restraints of $1000 \mathrm{~kJ} \mathrm{~mol}^{-1} \mathrm{~nm}^{-2}$ on the protein heavy atoms for $20 \mathrm{~ns}$. Subsequently, trajectories totaling in a length of $\sim 0.5 \mu$ s were obtained by free simulation.

To analyze slow and fast motions of mVDAC1, low- and high-pass filtering was applied on the trajectories. Calculated root mean square fluctuation (RMSF) values were transformed into isotropic B-factors and averaged using a running average of eight residues length. A contribution of $1 \AA$ to the total RMSF was assumed to arise from static disorder and subtracted.

Collective motions of mVDAC1 were analyzed by performing a principal component analysis (PCA) on the barrel backbone (amino acids 21-283) and projecting the trajectories on the obtained eigenvectors. Elliptic fitting was carried out on eigenmode 1 using $\mathrm{C} \alpha$ positions. All molecular representations were generated using VMD (Humphrey et al. 1996). The lipid bilayer thickness was determined by measuring the average distance between the phosphate atoms of DMPC in the lower and upper leaflets using a grid-based approach based upon the GridMAT algorithm (Allen et al. 2009). 



\section{Results}

\subsection{Functionality of recombinantly expressed hVDAC1}

hVDAC1 was recombinantly expressed, refolded in LDAO, and purified (see Appendix) as previously described (Bayrhuber et al. 2008; Engelhardt et al. 2007). In order to prove the functionality of our refolded hVDAC1 preparations in LDAO used for further dynamic and functional characterization, we measured conductance and voltage dependence of hVDAC1 incorporated into planar bilayer lipid membranes (BLMs) as described (Benz 1994). Micellar hVDAC1 inserted into the membrane (Figure 10A) and displayed the typical open state conductance of $4 \mathrm{nS}$ and closed state conductance of $2 \mathrm{nS}$ (Figure 10B) at a membrane potential of $10 \mathrm{mV}$ as well as voltage-dependent channel closure at voltages above $\sim \pm 25 \mathrm{mV}$ (Figure 10C).
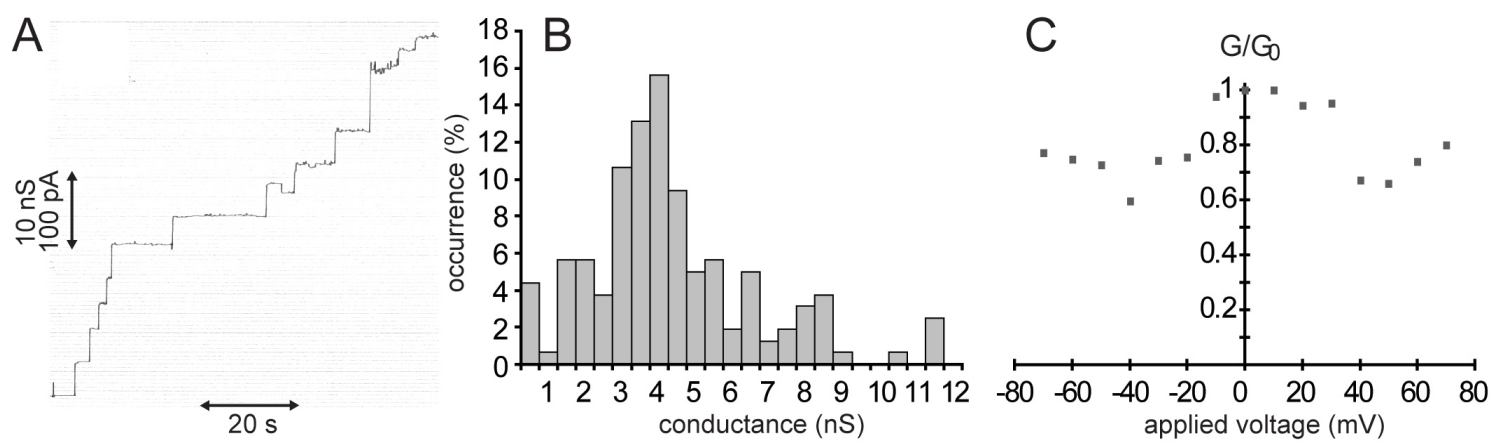

Figure 10. Electrophysiological properties of refolded hVDAC1 in bilayer lipid membranes (BLM). (A) Insertion of single hVDAC1 channels after addition to both sides of a diphytanoyl phosphatidylcholine/n-decane membrane in a $1 \mathrm{M} \mathrm{KCl}$ bathing solution. (B) Histogram of the channel conductance fluctuations of inserted hVDAC1 at a membrane potential of $10 \mathrm{mV}$. Major single-channel conductance levels are clustered at $4 \mathrm{nS}$ (open state), $2 \mathrm{nS}$ (closed state) and $8.5 \mathrm{nS}$ (simultaneous insertion of two VDAC channels). The number of total conductance increments was 160. (C) Voltagedependent closure of a hVDAC1 multi-channel membrane, given as the ratio of conductance $\mathrm{G}$, at a given voltage, divided by the conductance $\mathrm{G}_{0}$ at $10 \mathrm{mV}(\mathrm{n}=3)$. 
Due to the low number of experiments $(n=3)$, the voltage-dependent gating curve is not as symmetrical and smooth as expected. However, similar gating characteristics were also observed for VDAC isolated from native membranes of various species including hVDAC1 (Benz 1994; Blachly-Dyson et al. 1993). These data show that refolded micellar hVDAC1 is functional and displays properties in bilayers similar to those of native VDAC. 


\subsection{Improving the structure of hVDAC1 and the orientation of its N-terminal helix}

The main differences in the three mammalian VDAC1 structures occur in the structure and orientation of the N-terminal $\alpha$-helix. In our combined X-ray/NMR structure (Bayrhuber et al. 2008) residues 6-19 form a barrel-attached $\alpha$-helix. Similarly, in the crystal structure of mVDAC1 (Ujwal et al. 2008) residues 6-20 form a helix attached to the barrel. In addition, a kink was observed at residues L10-G11 in mVDAC1 but not our combined hVDAC1 structure. In both structures residues 20/21-25 form an unstructured linker to the first $\beta$-strand. In contrast, in the NMR structure (Hiller et al. 2008) only the first part of the helix (residues 6-10) forms a short $\alpha$-helix attached to the $\beta$-barrel, while the second part (residues 12-20) together with residues 21-25 forms a flexible disordered linker to the first $\beta$-strand. For simplicity, the topology of the mVDAC1 structure (defining the $\alpha$-helix and $\beta$-strands) is used throughout this work.

Since the helix is proposed to be involved in gating, protein interactions, and regulation of apoptosis (Abu-Hamad et al. 2009; Hiller and Wagner 2009; Ujwal et al. 2008), refinement of the structure in general, and of the helix in particular, is of major interest. An NMR or a combined NMR/X-ray structure can be improved by an increased amount of backbone resonance assignments and refinement of the structure with additional NMR restraints such as residual dipolar couplings (RDCs). The improved assignment and RDC data, as well as mutations and spin labels placed in the helix, also help to characterize dynamics, interactions and structural changes in the helix induced by solutes or interacting proteins.

\subsubsection{Improved backbone resonance assignment of hVDAC1}

Previously, a backbone resonance assignment of 70/90\% (overall/barrel) was achieved for hVDAC1 in micellar solution (Bayrhuber et al. 2008). With improved sample quality and additional NMR experiments (Figure 11), the assignment was further increased to $85 / 93 \%$ and $88 / 97 \%$ (overall/barrel) for $w t$ hVDAC1 and E73V hVDAC1, respectively (Figure 12). 

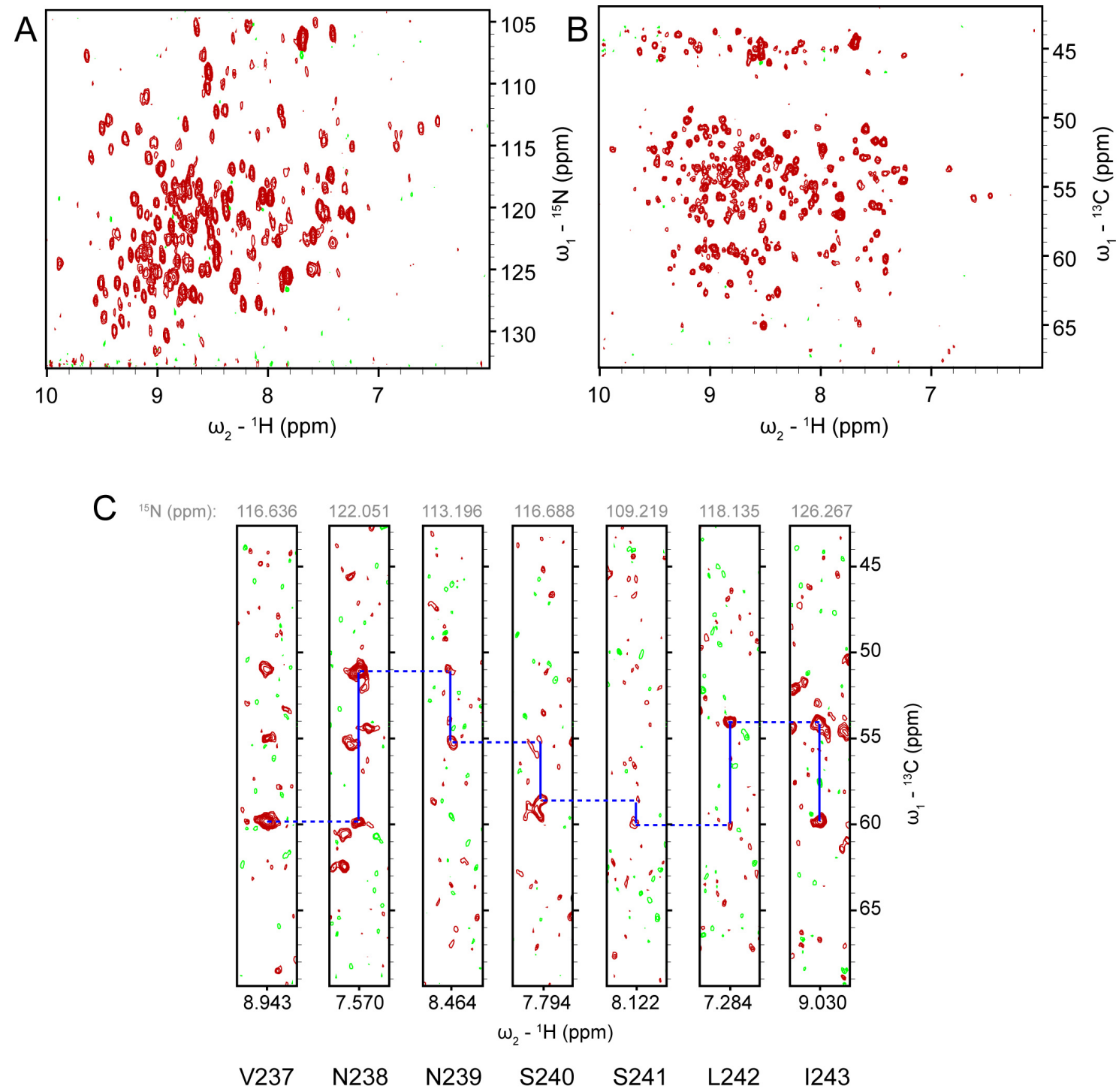

Figure 11. TROSY-HNCA spectrum of hVDAC1 with improved purity. $2 \mathrm{D}{ }^{1} \mathrm{H},{ }^{15} \mathrm{~N}-(\mathrm{A})$ and $2 \mathrm{D}$ ${ }^{1} \mathrm{H},{ }^{13} \mathrm{C}$ - (B) projections of a 3D TROSY-HNCA spectrum measured on $w t$ hVDAC1 with improved purity at a ${ }^{1} \mathrm{H}$ frequency of $900 \mathrm{MHz}$ and $37{ }^{\circ} \mathrm{C}$. (C) $2 \mathrm{D}{ }^{1} \mathrm{H},{ }^{13} \mathrm{C}$-strips of the same spectrum showing a sequential walk for the previously unassigned loop L16-17 and adjacent $\beta$-barrel edges (residues V237-I243).

The lower assignment level for $w t$ hVDAC1 compared to E73V hVDAC1 resulted from low signal intensities and missing $\mathrm{C} \alpha$ connectivities in the $\mathrm{N}$-terminal part of the $\beta$-barrel, which prevented the assignment of seven residues in $\beta 4$ (T72-W75) and $\beta 5$ (G82-E84) in $w t$ hVDAC1 (Figure 12). Resonance broadening can be a consequence of conformational exchange, and a detailed analysis of dynamics is provided in section 3.3 . 

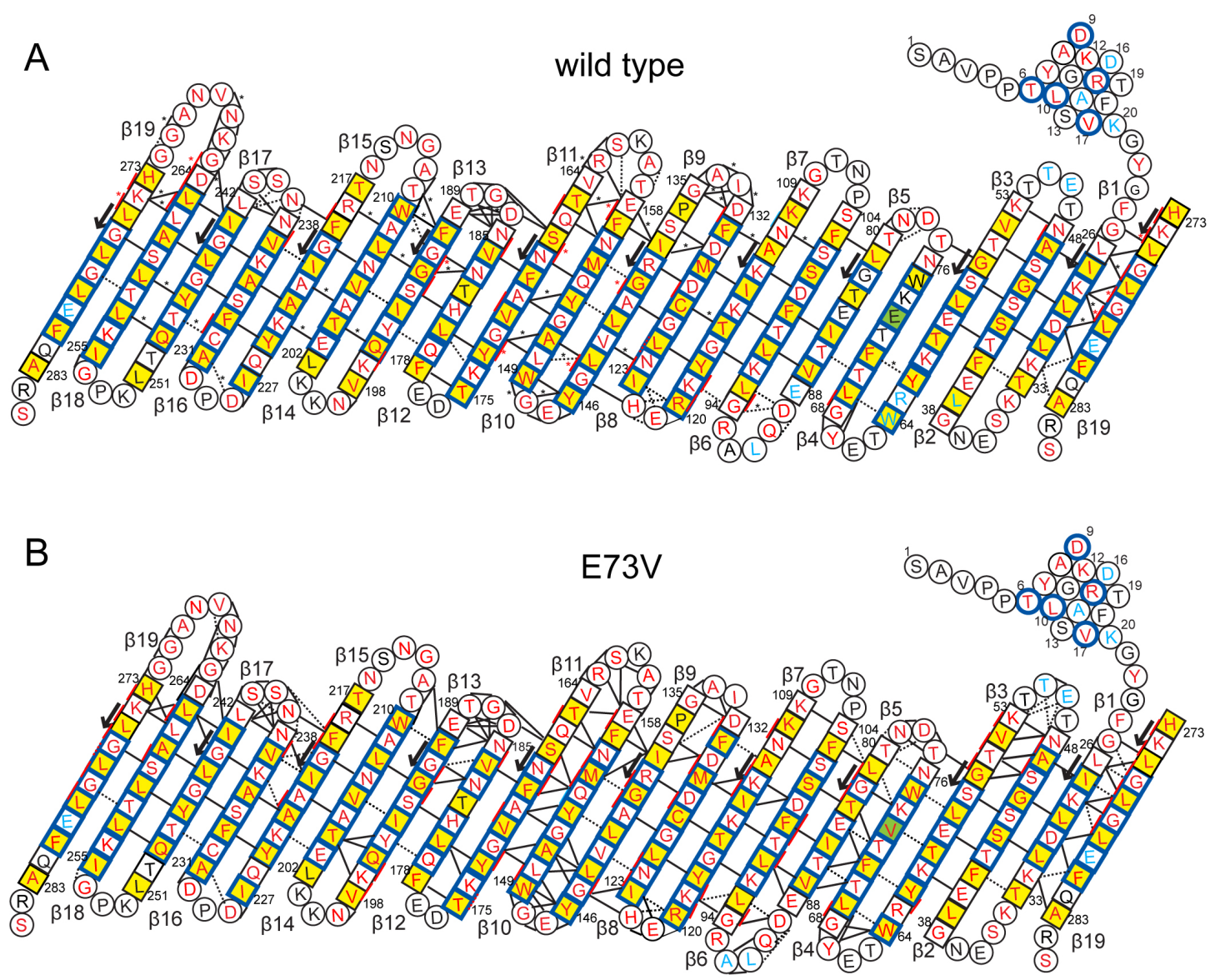

Figure 12. Backbone assignment and NOEs of hVDAC1. Improved backbone resonance assignment and $\mathrm{H}_{\mathrm{N}}-\mathrm{H}_{\mathrm{N}}$ NOEs in $w t$ (A) and E73V (B) hVDAC1 obtained from HMQC-NOESY spectra of $w t$, E73V and $\mathrm{V} 87 \mathrm{C} / \mathrm{C} 127 \mathrm{~S} / \mathrm{C} 232 \mathrm{~S}$ hVDAC1 at $37{ }^{\circ} \mathrm{C}$ and $800 \mathrm{MHz}$. The topology map of hVDAC1, adopted from the crystal structure of mVDAC1 (Ujwal et al. 2008), is shown with assigned residues (red), ambiguously assigned residues (blue), and unassigned residues (black). Residues in $\beta$-strands are boxed, yellow shading indicates outward pointing residues. The mutation site (E73 to V) is highlighted in green. Residues showing slow H/D exchange in E73V hVDAC1 ${ }^{1} \mathrm{H},{ }^{15} \mathrm{~N}$-TROSY spectra are framed in blue. Black (red) lines indicate inter-strand (sequential) $\mathrm{H}_{\mathrm{N}}-\mathrm{H}_{\mathrm{N}}$ NOEs. Dotted lines indicate (partially) overlapping NOEs. Asterisks indicate NOEs observed in HMQC-NOESY spectra of V87C/C127S/C232S but not $w t$ hVDAC1. The figure was adapted from (Bayrhuber et al. 2008).

In addition, the assignment of the N-terminal $\alpha$-helix was complicated by resonance broadening and missing $\mathrm{C} \alpha$ connections and NOEs in both $w t$ and E73V hVDAC1, indicating the presence of a certain extent of conformational exchange. Five residues in the first part of the helix (T6-L10) and three residues in the second helical part (A14, R15, K20) had been assigned in solution NMR spectra previously. Residues A2-P5 could not be assigned unambiguously, due to the absence of amides in the two proline residues (P4, P5) and missing $\mathrm{C} \alpha$ connections. With the chemical shift information from solid-state NMR three additional residues $(\mathrm{K} 12, \mathrm{D} 16, \mathrm{~V} 17)$ in the 
second part of the helix (residues 12-20) were assigned (Figure 12 and Table 5), showing that the second part of the $\alpha$-helix is not completely exchange broadened or unstructured in solution. This result agrees with the observation that peaks corresponding to residues A2-V17 give rise to distinct narrow cross peaks in solid-state NMR spectra of hVDAC1 reconstituted in liposomes, indicating a rather rigid conformation of the $\alpha$-helix (Schneider et al. 2010). The only disagreement between solution and solid state shifts is found for A14, were the weak $\mathrm{C} \alpha(\mathrm{i}-1)$ resonance does not match with the chemical shift of S13 obtained from solid state NMR (Table 5). V17 exhibits a very high $\mathrm{C} \alpha$ shift, making its assignment reliable despite missing NOEs and $\mathrm{C} \alpha$ connections and overlap in ${ }^{1} \mathrm{H},{ }^{15} \mathrm{~N}$-TROSY spectra (Table 5).

Table 5. Chemical shifts of the N-terminal $\alpha$-helix and linker.

\begin{tabular}{|c|c|c|c|c|}
\hline \multirow[t]{2}{*}{ Residue } & \multicolumn{2}{|c|}{ Solid state NMR } & \multicolumn{2}{|c|}{ Solution $\mathrm{NMR}^{\perp}$} \\
\hline & ${ }^{15} \mathrm{~N}_{\mathrm{H}}(\mathrm{ppm})$ & ${ }^{13} \mathrm{C} \alpha(\mathrm{ppm})$ & ${ }^{15} \mathrm{~N}_{\mathrm{H}}(\mathrm{ppm})$ & ${ }^{13} \mathrm{C} \alpha(\mathrm{ppm})$ \\
\hline M1 & - & - & - & - \\
\hline A2 & - & 51.2 & - & - \\
\hline V3 & - & 60.0 & - & - \\
\hline $\mathrm{P} 4$ & 122.0 & 61.6 & - & - \\
\hline P5 & - & 61.5 & - & - \\
\hline T6 & 106.7 & 60.5 & 106.2 & 60.4 \\
\hline Y7 & - & 62.5 & 122.6 & 62.0 \\
\hline A8 & 118.7 & 54.1 & 119.0 & 53.8 \\
\hline D9 & 114.5 & 54.0 & 115.2 & 53.8 \\
\hline L10 & - & 56.3 & 125.4 & 56.1 \\
\hline G11 & 114.9 & 45.1 & - & $44.9^{*}$ \\
\hline K12 & 123.5 & 60.1 & 122.4 & 59.9 \\
\hline $\mathrm{S} 13$ & 115.5 & 60.0 & - & $63.0 *$ \\
\hline A14 & 121.8 & 55.5 & (122.0) & $(55.2)$ \\
\hline $\mathrm{R} 15$ & 116.6 & 60.4 & 117.3 & 60.0 \\
\hline D16 & 118.1 & 57.1 & (118.6) & $(57.1)^{\#}$ \\
\hline V17 & 120.4 & 66.8 & 120.4 & 66.6 \\
\hline F18 & - & - & - & - \\
\hline T19 & - & - & - & $56.2 *$ \\
\hline K20 & - & - & $(122.0)$ & $(60.8)$ \\
\hline G21 & - & - & - & - \\
\hline Y22 & - & - & 113.7 & 56.0 \\
\hline G23 & - & - & - & - \\
\hline F24 & - & - & 120.3 & 56.4 \\
\hline G25 & - & - & 110.3 & 45.3 \\
\hline
\end{tabular}

† solid state NMR chemical shift values were obtained from Robert Schneider (Schneider et al. 2010)

$\perp$ Spectra were referenced by NMRPipe (Delaglio et al. 1995) and shifted by an additional +0.3 ppm in the ${ }^{13} \mathrm{C}$ dimension to improve the match with solid state shifts for residues T6-L10.

* shift obtained from C $\alpha(i-1)$ resonance; \# shift from E73V hVDAC1 spectra

- $\quad$ not assigned; ambiguous assignments are given in brackets 
In contrast, the assignments of D16 and K20 are ambiguous due to missing sequential connections and NOEs.

Secondary chemical shifts for $\mathrm{C} \alpha$ and $C^{\prime}$ resonances indicate that residues 7 (and potentially also residue 8), 12-17, and 20 adopt a helical conformation (Figure 13). In contrast, residues 9-11 form a kink in the $\alpha$-helix, residues 6,22 , and 24 adopt an extended conformation, and residue 25 adopts a random coil conformation (Figure 13).

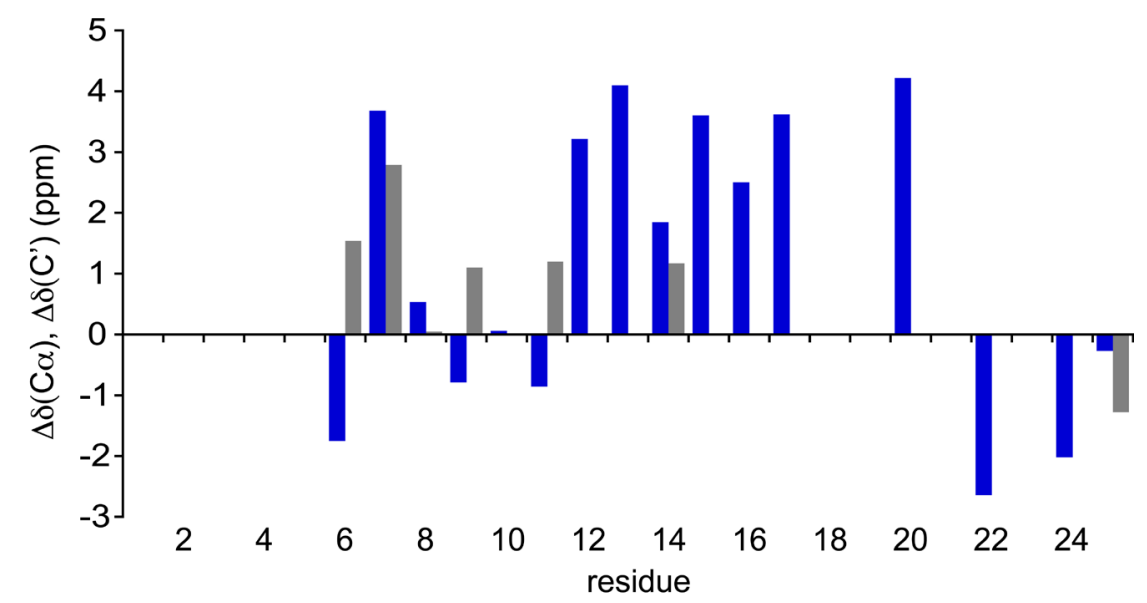

Figure 13. Secondary chemical shift for resonances in the $\mathbf{N}$-terminal $\alpha$-helix and linker. $\mathrm{C} \alpha$ and $\mathrm{C}^{\prime}$ secondary chemical shifts $\left(\Delta \delta(C \alpha)\right.$, blue) and $\left(\Delta \delta\left(C^{\prime}\right)\right.$, grey) for residues 1-25. C $\alpha$ resonances were obtained directly from the same residue (i) or indirectly from the (i-1) resonances of the following residue from a 3D TROSY-HNCA spectrum of $w t$ or E73V hVDAC1. C' resonances were obtained from the (i-1) resonance of the following resonance in a 3D TROSY-HNCO spectrum of $w t$ hVDAC1. Spectra were referenced automatically by the program NMRPipe (Delaglio et al. 1995) and an additional shift of 0.3 ppm was applied to the ${ }^{13} \mathrm{C}$ dimension to increase the match between chemical shifts in solution and the solid state.

In summary, 14 out of the $25 \mathrm{~N}$-terminal residues could be assigned (with three ambiguous assignments: A14, D16, and K20). Furthermore, the combined data indicate a kinked helical structure that was also observed for hVDAC1 in liposomes (Schneider et al. 2010) and the mVDAC1 crystal structure obtained from bicelles (Ujwal et al. 2008). Furthermore, ${ }^{1} \mathrm{H},{ }^{15} \mathrm{~N}$-TROSY spectra of hVDAC1 show about 40 unassigned resonances, while 36 residues of hVDAC1 are still unassigned. Therefore it is likely that some of these 40 residues correspond to cross peaks of $\alpha$-helix and linker residues, which could not be assigned so far due to low intensity, overlap, or lack of C $\alpha$ connections and NOEs. Other techniques, such as selective labelling schemes, are required to assign further $\alpha$-helix/linker residues. 


\subsubsection{Probing the orientation of the $\alpha$-helix}

In order to study the structure and location of the $\alpha$-helix/linker under various conditions, mutations were created in the helix of hVDAC1. To follow large structural changes of the $\alpha$-helix under certain conditions, for instance a metabolite induced motion of the second $\alpha$-helical part into the $\beta$-barrel, a cysteine residue at the $\mathrm{C}$ terminal end of the $\alpha$-helix (residue 19) was designed and an MTSL spin label was attached. This enables determination of helix motion from changes in the PRE profile for residues in the $\beta$-barrel upon addition of metabolites. In order to exclude possible disulfide bridge formation within the protein, the two intrinsic cysteines (C127 and C232) were removed by mutation to serine. The cysteine-free C127S/C232S hVDAC1 yielded NMR spectra of equal quality to $w t$ hVDAC1 (Bayrhuber et al. 2008), showing that the two cysteines are not essential for the structure or stability of hVDAC1. Furthermore, cysteine-free rat VDAC1 (rVDAC1) shows electrophysiological characteristics as the native protein (Aram et al. 2010). In contrast, attachment of the spin label to $\mathrm{T} 19 \mathrm{C} / \mathrm{C} 127 \mathrm{~S} / \mathrm{C} 232 \mathrm{hVDAC} 1$ induced unfolding and aggregation of the protein. This result is most likely due to a disruptive force of the hydrophobic spin label placed at a solvent exposed position. In addition, spin label free T19C/C127S/C232S hVDAC1 formed stable disulfide bridged dimers, as judged from the poor spectral quality and elevated rotational correlation time (50-60 ns compared to 20-30 ns for $w t$ hVDAC1), estimated from a ${ }^{1} \mathrm{H} 1 \mathrm{D}$ (1-1) echo experiment (Sklenář and Bax 1987) (data not shown). This indicates that the $\alpha$-helix is not stably attached to the $\beta$-barrel, but accessible to the $\alpha$-helix from another hVDAC1 monomer. However, it is unclear if the introduced cysteine residue induces this accessibility by changing the $\alpha$-helix structure. Thus, mutation at this position introduced artefacts that prevented meaningful studies of the $\alpha$-helix structure and orientation.

Nevertheless, the mutation provides additional information for the assignment of

$\alpha$-helix and linker residues. A comparison of ${ }^{1} \mathrm{H},{ }^{15} \mathrm{~N}$-TROSY spectra of T19C/C127S/C232S with C127S/C232S hVDAC1 clearly demonstrates the presence of yet unassigned residues of the $\alpha$-helix and the linker (Figure 14). 


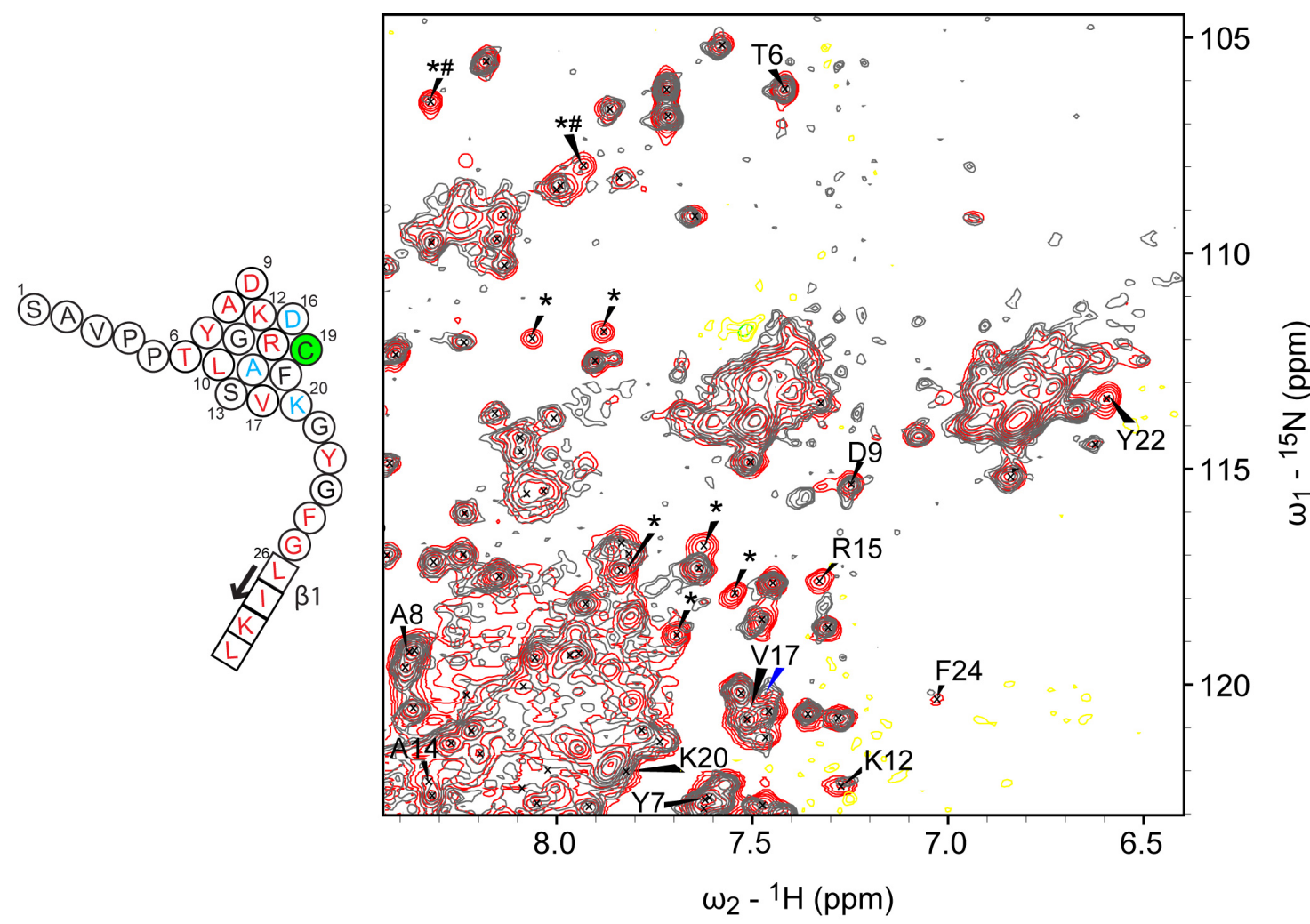

Figure 14. Mutation of T19 confirms resonance assignments in the $\boldsymbol{\alpha}$-helix and linker. On the left the topology of the $\alpha$-helix and the linker (encircled residues), connecting the helix to the first $\beta$-strand $\beta 1$ (boxed residues), is shown. Assigned residues are printed in red, unassigned residues in black, ambiguous assignments are blue. The mutation in T19C is highlighted in green. The right panel shows an overlay of ${ }^{1} \mathrm{H},{ }^{15} \mathrm{~N}-\mathrm{TROSY}$ spectra of $\mathrm{C} 127 \mathrm{~S} / \mathrm{C} 232 \mathrm{~S}$ hVDAC1 (reference, red) and T19C/C127S/C232S hVDAC1 (grey). Assigned residues in the helix or linker region are labelled. Unassigned residues shifting in the mutant are labelled with an asterisk. Cross-peaks corresponding to glycine residues are marked (\#). The new position of V17, which is overlapped but most likely shifting in the mutant spectra, is indicated with a blue arrow.

Due to the lack of NOEs and C $\alpha$ connections the assignment problem for the second $\alpha$-helical part and the linker remained, although $\mathrm{C} \alpha$ and ${ }^{15} \mathrm{~N}_{\mathrm{H}}$ chemical shift information from solid state NMR was taken into consideration. However, the mutationinduced spectral changes provide support for many assignments of the $\alpha$-helix/linker residues, as resonances close to the mutation site (K12, A14, R15, V17, Y22, and F24) shifted or were broadened (Figure 14). In addition, two glycine resonances could be identified that shifted or were broadened beyond detection in T19C/C127S/C232S hVDAC1 (Figure 14). However, their $\mathrm{C} \alpha$ chemical shifts are are not matching the anticipated chemical shifts for G21 and G23, suggesting that there might be two or more sets of resonances for the linker residues (G21, Y22, G23, F24). For G25 a second resonance is frequently observed. 
Taken together, these data agree with the current $\alpha$-helix and linker assignments and indicate the presence of slow conformational exchange in the linker (G21-G25). The exchange might influence the second part of the $\alpha$-helix (residues 12-20) as well and complicates the assignment and structural analysis of the $\alpha$-helix/linker.

\subsubsection{Helix destabilization affects hVDAC1 in a complex manner}

The agreement of solution and solid state chemical shifts for the assigned $\alpha$-helix residues indicates rigidity in the $\alpha$-helix, while low resonance intensities and missing cross-peaks suggest a certain extent of conformational exchange. Therefore, we investigated the effect of introducing further perturbations in the $\alpha$-helix. R15 was proposed to stabilize the 2-segment-helix observed in the crystal structure of mVDAC1 by formation of a bidentate hydrogen bond to A8 and L10 (Ujwal et al. 2008). Since the solution and solid state data indicate a similar $\alpha$-helical structure, such stabilization potentially occurs in solution. Thus, disruption of the hydrogen bonds should increase flexibility in the helix and might also affect the stability of the whole barrel structure in solution. Therefore we introduced R15A as a mutation in the helix. The R15A mutation induced shifts and broadening in the entire protein (Figure 15). Strong shifts were observed in the helix (Y7, A8, D9, L10, K12, R15 (mutation site), D16 (most likely shifted), V17 (most likely shifted)), thus confirming most of the $\alpha$-helix assignments. Interestingly, A14 shifted only modestly, despite its close proximity to the mutation site (Figure 15A).

In addition to shifts in the helix, residues in $\beta$-strands $\beta 9-\beta 19$ (V143, N156, R163, N168, G172, T175, Q179, L180, H181, N185, D186, G191, G192, S193, I194, Q196, N207, L208, A209, G213, G220, A222, D230, A231, G244, Q249, G254, I255, T258, D264, G271, S285) experienced chemical shift changes (Figure 15A). Many of the shifting residues are in proximity to the mutation site in the $3 \mathrm{D}$ structure $(\beta$-barrel residues in proximity to the $\alpha$-helix are highlighted in bold). Hence, the $\alpha$-helix is not completely mobile as suggested by the NMR structure (Hiller et al. 2008), but at least partially attached to the barrel wall in solution. This result is in line with the similarity in chemical shifts in solution and the solid state (Table 5) (Schneider et al. 2010). 

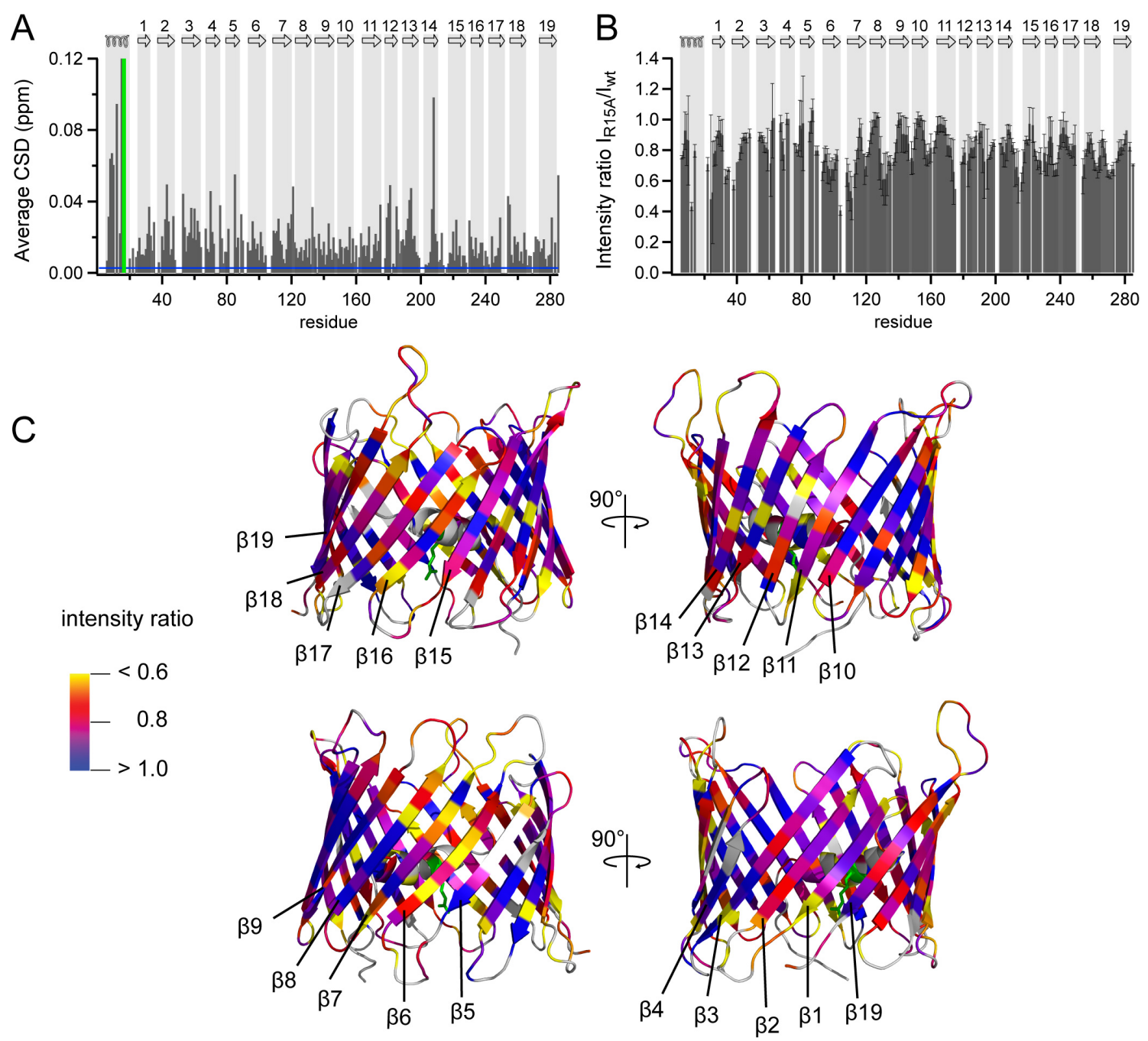

Figure 15. $\alpha$-helix destabilization has long-range effects on the structure of hVDAC1. (A) Average chemical shift deviations (CSD) of R15A hVDAC1 compared to $w t$ hVDAC1. The blue line indicates the average CSD for non-shifting residues. Green data points indicate residues D16 and V17, which are most likely shifted. (B) Resonance intensity ratio $\left(\mathrm{I}_{\mathrm{R} 15 \mathrm{~A}} / \mathrm{I}_{\mathrm{wt}}\right)$, averaged over a three-residue window (mean \pm standard error). (C) Cartoon representations of hVDAC1 (PDB code: 2JK4) with intensity ratios mapped. $\beta$-strands are numbered. R15 is shown as green sticks. Non-resolved residues are mapped in white.

Moreover, the CSD observed in many $\beta$-barrel residues not immediately in contact with R15 suggests long-range effects on the $\alpha$-helix and on the adjacent barrel wall.

Interestingly, strong shifts were also observed in the N-terminal barrel region (L31, K32, T33, S35, E40, T42, S43, S44, A47, K53, V54, G56, S57, L58, T60, Y62, R63, Y67, T70, F71, N76, L81, I85, D89, K96, S101, K109, K110, N111, Y118, K119, R120, E121) (Figure 15A). Moreover, resonance intensities changed in a complex pattern upon mutation of R15 (Figure 15B). While absolute scaling of the ratios 
between R15A and $w t$ hVDAC1 was not possible due to variations in the sample quality, detergent concentration, and experimental setup (receiver gain), the strongest relative broadening appeared in the C-terminal $\beta$-strands $\beta 12-\beta 19$ (involved in helix interaction), K12 in the helix, the linker (K20, Y22, F24), and in the N-terminal $\beta$ strands $\beta 6-\beta 8$ (Figure 15B, C). A stronger resonance broadening is in line with increased conformational exchange on the micro- to millisecond time scale.

Thus, the data suggest that (i) R15 is involved in stabilization of the $\alpha$-helix (in particular the connection of the first and second helical parts), the linker, and nearby barrel regions and (ii) also influences the structure or stability of the opposite Nterminal barrel region $(\beta 6-\beta 8)$. The latter observation could be due to two reasons. Firstly, the $\alpha$-helix stabilizes the $\beta$-barrel by interactions. Consequently, an increased flexibility of the mutated $\alpha$-helix would lead to destabilization of the whole $\beta$-barrel. This assumption is in line with enhanced dynamics or aggregation of the truncated VDAC, when the complete $\alpha$-helix is removed (unpublished observations). Similarly, solid state NMR revealed that $\alpha$-helix truncation results in instability of $\beta$-strand $\beta$, which is in contact with the first helical segment (residues 6-10) (Schneider et al. 2010). Secondly, the second part of the $\alpha$-helix (residues 12-20) transiently approaches the Nterminal $\beta$-barrel region as suggested by the NMR structure of hVDAC1 (Hiller et al. 2008) and thereby induces the observed shifts and intensity changes in this region. Such a mechanism is in line with potential flexibility in the second part of the $\alpha$-helix and linker that is suggested by low peak intensities, missing $\mathrm{C} \alpha$ connections, and NOEs (Figure 12), as well as the potential second resonance set for the linker (Figure 14).

\subsubsection{Alignment of hVDAC1 in orienting media}

RDCs from samples aligned in the magnetic field are of great value for the refinement of an NMR structure, because they provide long-range structural information (Lipsitz and Tjandra 2004). In the case of hVDAC1, RDCs could prove valuable for the determination of the structure and orientation of the N-terminal $\alpha$-helix in solution under varying conditions. By this approach dynamics and structural changes of the $\alpha$ helix can be investigated in light of its role in gating. Alignment of membrane proteins is hampered by the requirement of a solubilizing detergent, which destroys most of the 
alignment media available for soluble proteins. Some alignment media forming liquid crystalline phases are stable in the presence of certain detergents (Lorieau et al. 2008; Park et al. 2009). While the stability of liquid crystalline phases in other detergents likely depends on the detergent harshness, the alignment in fixed physical barriers (e.g. polyacrylamide gels (Cierpicki and Bushweller 2004)) is independent of the detergent used. Unlike certain paramagnetic tags (Ikegami et al. 2004), these alignment media do not induce pseudocontact shifts nor require new resonance assignment. Therefore we tested the usefulness of two different liquid crystalline media and charged polyacrylamide gels for alignment of hVDAC1 in LDAO micelles.

\subsubsection{LDAO disrupts liquid crystalline phases}

A suitable alignment medium for membrane proteins solubilized in the detergent dodecylphosphocholine (DPC) are the so called "G-tetrads" formed by 2'deoxyguanylyl(3' $\left.\rightarrow 5^{\prime}\right)-2^{\prime}$-deoxyguanosine $(\mathrm{dGpG})$ at DPC concentrations of up to $200 \mathrm{mM}$ (Lorieau et al. 2008). In complex with potassium or sodium ions dGpG stacks as G-tetrads, which form a liquid crystalline phase in a magnetic field (Davis 2004). We tested whether the liquid crystalline phase is stable in LDAO solutions and therefore suitable for hVDAC1 preparations. In detergent-free buffer the residual quadrupolar splitting was stable over a temperature range of 298-310 K (Figure 16A).
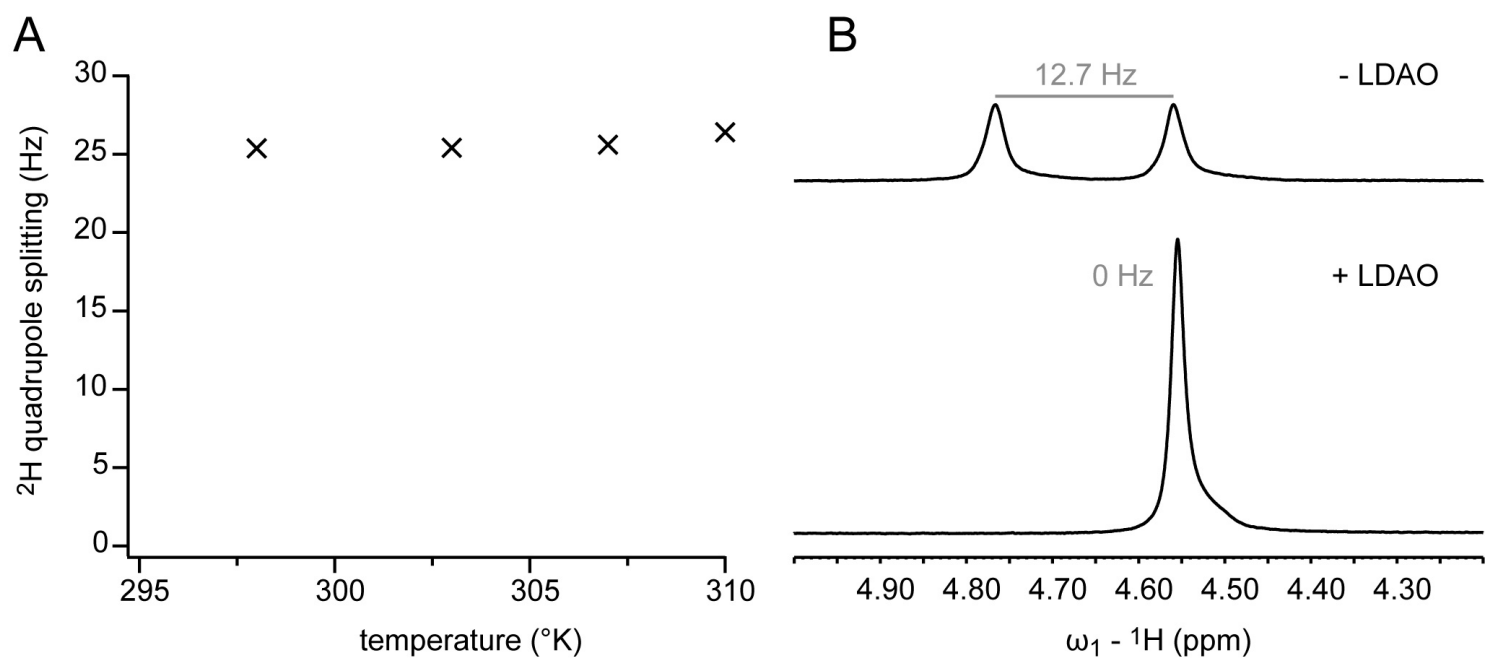

Figure 16. LDAO disrupts the dGpG induced alignment. Residual ${ }^{2} \mathrm{H}$ quadrupole splitting in a solution of $35 \mathrm{mg} / \mathrm{ml} \mathrm{dGpG}$ at different temperatures (A) and of $11 \mathrm{mg} / \mathrm{ml} \mathrm{dGpG}$ before and after the addition of $1 \%$ (44 $\mathrm{mM})$ LDAO (B). 
In contrast, low LDAO concentrations disrupted the alignment (Figure 16B). The disruption of alignment could not be rescued by higher potassium ion concentrations (up to $160 \mathrm{mM}$ ) or by dissolving the medium directly in detergent containing buffer (data not shown). A maximum splitting of $1 \mathrm{~Hz}$ was achieved, when the $\mathrm{dGpG}$ concentration was raised to $60 \mathrm{mg} / \mathrm{ml}$. However, at this high concentration the sample viscosity was very high and proper handling of the medium impossible.

Based on the finding that alignment of membrane proteins can be achieved with fd bacteriophages in the presence of another detergent, 1,2-dihexanoyl-sn-glycero-3phosphocholine (DHPC), at $100 \mathrm{mM}$ concentrations (Park et al. 2009), and the similarity of fd phages to the more commonly used Pf1 phages (Torbet and Maret 1981), we tested the stability of the liquid crystalline phase formed by Pf1 phages in LDAO-containing solution. In the absence of detergent the residual ${ }^{2} \mathrm{H}$ quadrupole splitting occurred in a phage concentration dependent manner, showing the presence of alignment in the sample (Figure 17A, B). Addition of LDAO at concentrations lower than the amount usually present in hVDAC1 NMR samples led to a dramatic increase in viscosity of the sample and disrupted the alignment (Figure 17C, D).

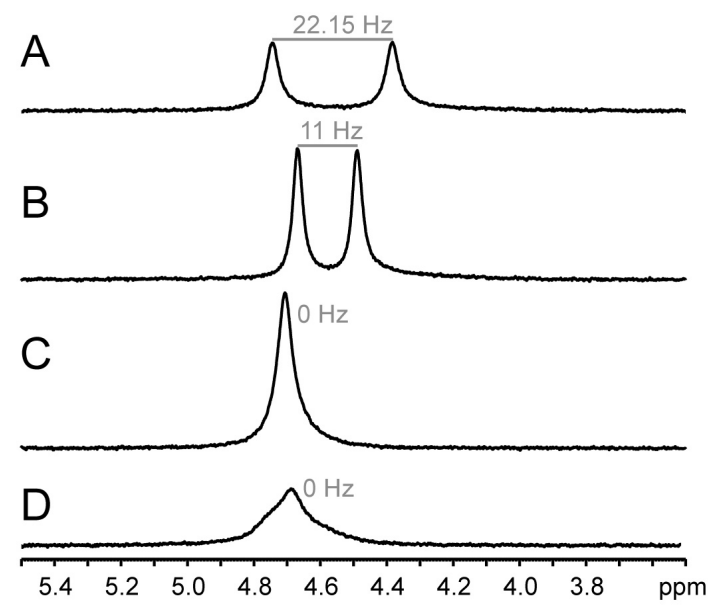

Figure 17. LDAO disrupts the Pf1 phage induced alignment. $1 \mathrm{D}{ }^{2} \mathrm{H}$ NMR spectra showing the residual ${ }^{2} \mathrm{H}$ quadrupole splitting (grey) measured for the following sample conditions: $16.7 \mathrm{mg} / \mathrm{ml}(\mathrm{A})$ and $8.35 \mathrm{mg} / \mathrm{ml}$ (B) Pf1 phages in the absence of LDAO; $8.2 \mathrm{mg} / \mathrm{ml}$ phages in the presence of $0.5 \%(22 \mathrm{mM})$ LDAO (C); $12.5 \mathrm{mg} / \mathrm{ml} \mathrm{Pfl}$ phages in the presence of $0.25 \%$ (11 mM) LDAO (D). In (D) low magnetic field homogeneity caused by air bubbles in the gel-like phage/LDAO suspension lead to a distorted peak shape. 
In conclusion, neither of the two liquid crystalline alignment media that are stable in DPC or DHPC tolerated LDAO as a detergent. This result is likely related to the harshness of LDAO, which is similar to SDS. In order to use the liquid crystalline phases for alignment of hVDAC1, detergent exchange into DPC or DHPC was tested by protein precipitation and resolubilization in the respective detergent. By this preparation procedure only insufficient $(50 \mu \mathrm{M})$ concentrations of folded hVDAC1 could be solubilised in DHPC, while hVDAC1 was completely unfolded in DPC. Since detergent exchange via this procedure was not feasible, a different detergent exchange procedure (e.g. direct refolding or on-column detergent exchange) or an LDAO resistant alignment medium is required for alignment of $\mathrm{hVDAC} 1$.

\subsubsection{Alignment of hVDAC1 in charged polyacrylamide gels}

In contrast to liquid crystalline media, polymerized acrylamide gels are stable in the presence of any detergent and proved successful for RDC measurements of the bacterial OmpA (Cierpicki and Bushweller 2004; Cierpicki et al. 2006). Therefore we tested alignment of hVDAC1 in three types of charged polymerized gels: two positively charged gels with a $50 \%$ or $75 \%$ fraction of charged substance $(50+\mathrm{M}$ and $75+\mathrm{M}$, respectively), as well as a negatively charged gel containing $50 \%$ charged substance (50-S). In ${ }^{1} \mathrm{H},{ }^{15} \mathrm{~N}$-TROSY spectra the $75+\mathrm{M}$ gels performed best in terms of signal intensity in the folded region above an ${ }^{1} \mathrm{H}$ chemical shift of $8.5 \mathrm{ppm}$, while the negatively charged 50-S gel showed no intensity in the same region. Hence, a $75+\mathrm{M}$ gel was soaked with $0.6 \mathrm{mM}$ of hVDAC1, compressed to $13 \mathrm{~mm}$ (final gel concentration: $5.2 \%$ ) and ${ }^{1} \mathrm{H},{ }^{15} \mathrm{~N}$-TROSY and ${ }^{1} \mathrm{H},{ }^{15} \mathrm{~N}$-HSQC spectra were recorded in an interleaved fashion in order to extract halved ${ }^{1} \mathrm{H}^{-15} \mathrm{~N}$ residual dipolar couplings $\left({ }^{1} \mathrm{D}_{\mathrm{NH}}\right.$ ) (Kontaxis et al. 2000). At this strong compression level the quality of both spectra was poor, most likely due to interaction of hVDAC1 with the gel. Release of compression considerably improved the signal-to-noise ratio in the amide proton region above $8.5 \mathrm{ppm}$, indicating that the broadening was indeed due to interaction of hVDAC1 with the gel matrix (Figure 18A). Still, the quality of ${ }^{1} \mathrm{H},{ }^{15} \mathrm{~N}$-HSQC spectra was poor under these conditions (Figure 18B). 


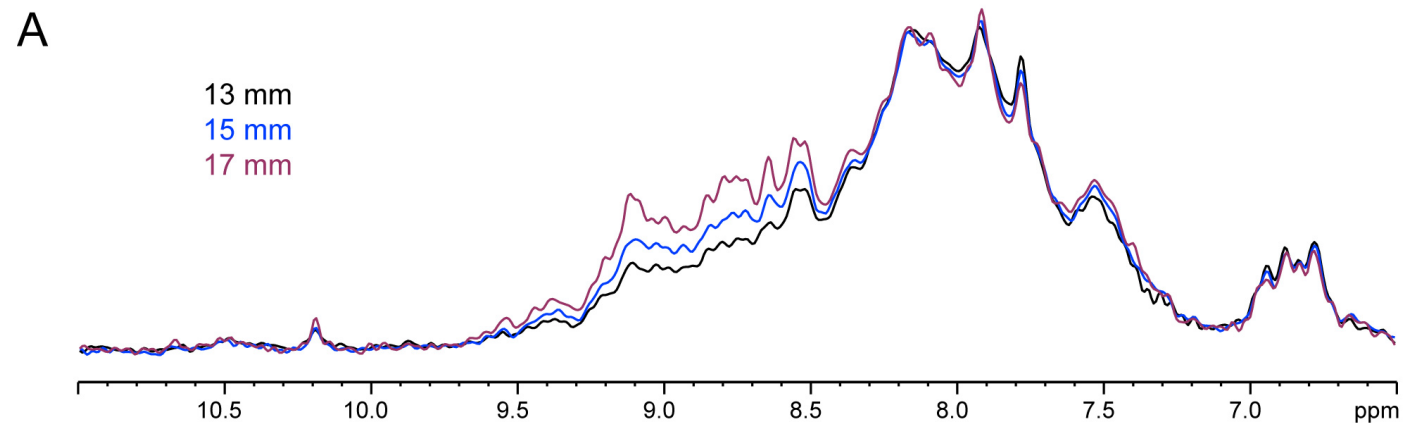

B
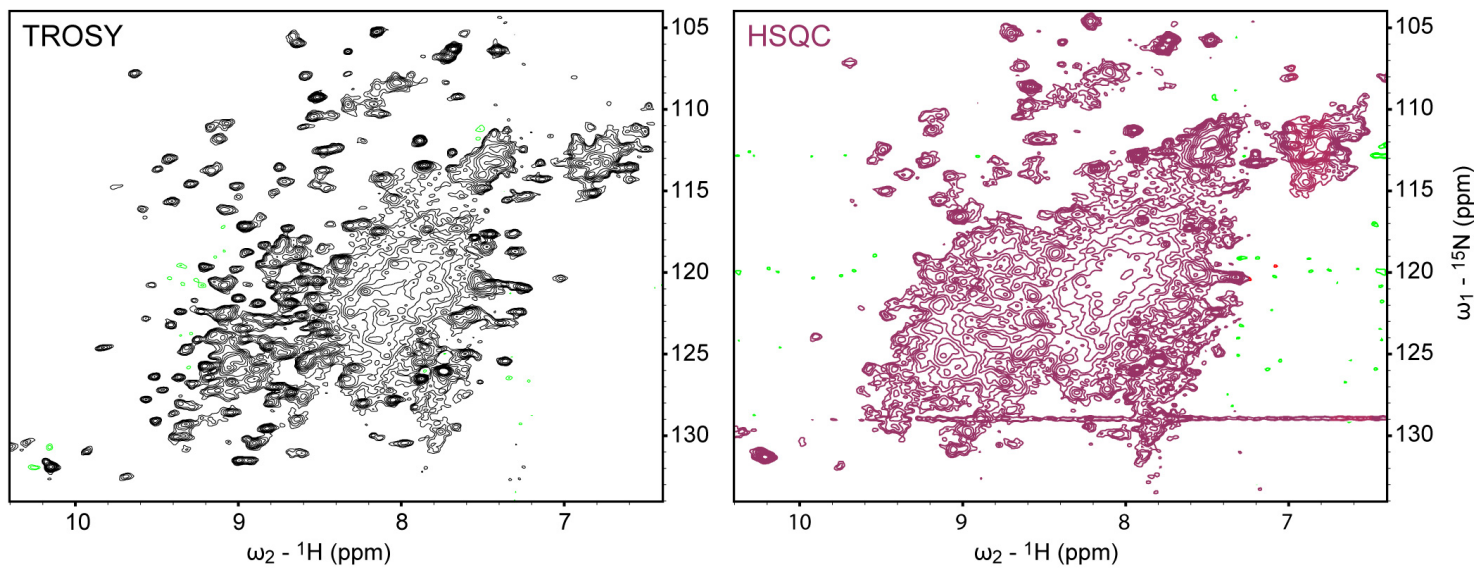

Figure 18. ${ }^{1} \mathbf{H},{ }^{15} \mathrm{~N}$-TROSY and ${ }^{1} \mathrm{H},{ }^{15} \mathrm{~N}$-HSQC spectra of hVDAC1 in a $75+\mathrm{M}$ gel. (A) $1 \mathrm{D}$ slices of ${ }^{1} \mathrm{H},{ }^{15} \mathrm{~N}$-TROSY spectra of C232S hVDAC1 soaked into a $75+\mathrm{M}$ gel and compressed to 13,15 , and 17 $\mathrm{mm}$ (gel concentrations of $5.2 \%, 4.5 \%$ and $4 \%$, respectively). (B) ${ }^{1} \mathrm{H},{ }^{15} \mathrm{~N}-\mathrm{TROSY}$ (black) and HSQC (red) spectra of $0.6 \mathrm{mM} \mathrm{C} 232 \mathrm{~S}$ hVDAC1 soaked into a 75+M polyacrylamide gel and compressed to 17 $\mathrm{mm}$. The spectra were recorded in an interleaved acquisition mode and extracted and processed using nmrPipe (Delaglio et al. 1995). The artefact in the HSQC spectrum results from strong signals of the polymerized charged acrylamide gel.

An alternative approach that applies a scaled $\left({ }^{1} \mathrm{~J}_{\mathrm{NH}}+{ }^{1} \mathrm{D}_{\mathrm{NH}}\right)$ splitting (Arbogast et al. 2010; Kontaxis et al. 2000; Yang et al. 1999) was also tested, in form of a 2D "RDC-TROSY" spectrum (Bhattacharya et al. 2010). However, in our hands variations of isotropic J-couplings were considerably larger when determined from the RDCTROSY than from interleaved TROSY/HSQC spectra (see Appendix). This result might be due to interference of the fractional deuteration or to the size of the VDAC-micelle complex exceeding the limits of the experiment. Thus, the interleaved TROSY/HSQC spectrum is currently the best choice for measurements of ${ }^{1} \mathrm{D}_{\mathrm{NH}}$ on hVDAC1.

From the interleaved spectra, $96{ }^{1} \mathrm{D}_{\mathrm{NH}}$ values were extracted that ranged from -35 to $+25 \mathrm{~Hz}$ (Figure 19A). Further filtering in terms of overlap and signal-to-noise reduced the set to $71 \mathrm{RDC}$ values of medium to high reliability. 
A

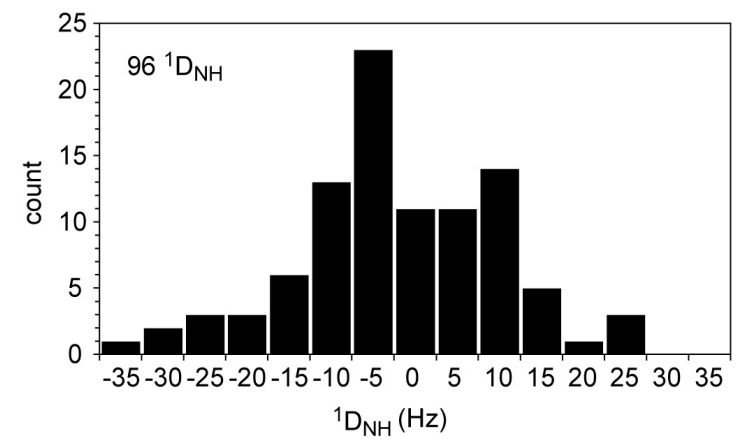

C

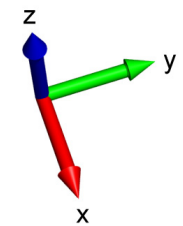

B

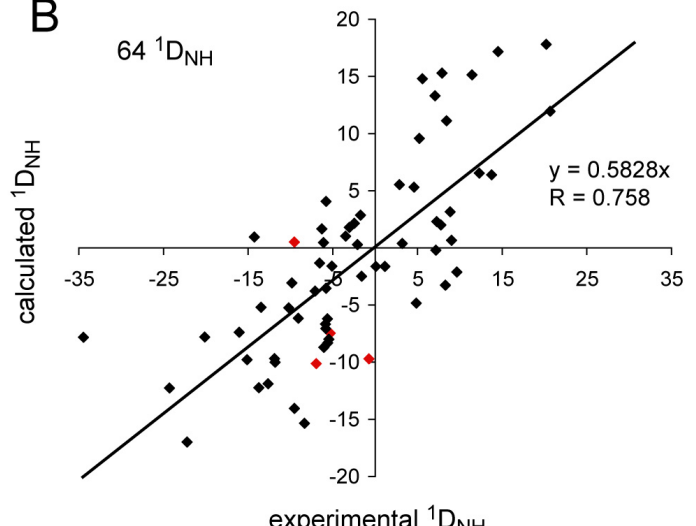

Figure 19. ${ }^{1} \mathrm{D}_{\mathrm{NH}}$ values from interleaved ${ }^{1} \mathrm{H},{ }^{15} \mathrm{~N}$-TROSY and ${ }^{1} \mathrm{H},{ }^{15} \mathrm{~N}-\mathrm{HSQC}$ spectra. (A) ${ }^{1} \mathrm{D}_{\mathrm{NH}}$ values were determined from the difference of ${ }^{15} \mathrm{~N}$ peak positions between ${ }^{1} \mathrm{H},{ }^{15} \mathrm{~N}-\mathrm{TROSY}$ and ${ }^{1} \mathrm{H},{ }^{15} \mathrm{~N}$-HSQC spectra, where a value of $92 \mathrm{~Hz}$ was used as ${ }^{1} \mathrm{~J}_{\mathrm{NH}}$ for all residues. (B) Correlation plot of the final set of calculated versus experimental ${ }^{1} \mathrm{D}_{\mathrm{NH}}$ values. Red diamonds indicate residues in the N-terminal helix. (C) Orientation of the alignment tensor resulting from the PALES fit of $64{ }^{1} \mathrm{D}_{\mathrm{NH}}$ values from (B) onto the crystal structure of mVDAC1 (PDB code: 3EMN).

These ${ }^{1} \mathrm{D}_{\mathrm{NH}}$ values were best-fit to the crystal structure of mVDAC1 (PDB code: 3EMN) using the PALES software (Zweckstetter 2008), resulting in a poor fit of experimental and calculated RDCs with a correlation (R) of 0.528 and a Q-factor of 0.812 (Table 6). Removal of experimental ${ }^{1} \mathrm{D}_{\mathrm{NH}}$ values of residues located in the loop L18-19 (residues 265-272) considerably improved the fit, resulting in R and Q-factor values of 0.758 and 0.350 , respectively (Table 6 and Figure 19B). Since residues in loop L18-19 exhibited intense and well-dispersed signals in both spectra and are therefore expected to yield reliable RDC values, the data suggest flexibility or a different conformation of loop L18-19 in micellar solution as compared to the crystal structure of mVDAC1 from bicelles. This is a likely scenario, since the loop is very flexible on the ps-ns time scale (see section 3.3.2). Usually, loops and unstructured regions are excluded from the analysis of RDC values. 
Table 6. PALES results of the molecular alignment simulations of $\mathrm{C} 232 \mathrm{~S} h \mathrm{hDAC1}{ }^{1} \mathrm{D}_{\mathrm{NH}}$ using the crystal structure of mVDAC1.

\begin{tabular}{llll}
\hline Data & Complete set of RDCs & $\begin{array}{l}\text { Excluding loop } \\
\text { L18-19 (residues 265- } \\
269,271-272)\end{array}$ & $\begin{array}{l}\text { Excluding } \alpha \text {-helix } \\
\text { residues } \\
\text { (T6, D9, A14, R15) }\end{array}$ \\
\hline $\mathrm{N}$ & & 64 & 60 \\
Da_HN & 71 & 15.53 & 15.80 \\
Rhombicity & 0.79 & 0.26 & 0.26 \\
RMS & 9.273 & 7.043 & 7.022 \\
Chi2 & 244.193 & 126.98 & 118.346 \\
Corr R & 0.528 & 0.758 & 0.776 \\
Q Saupe & 0.812 & 0.350 & 0.343 \\
Regression offset & $0.333 \pm 0.618[\mathrm{~Hz}]$ & $0.334 \pm 0.703[\mathrm{~Hz}]$ & $0.585 \pm 0.730[\mathrm{~Hz}]$ \\
Regression slope & $0.291 \pm 0.056[\mathrm{~Hz}]$ & $0.590 \pm 0.064[\mathrm{~Hz}]$ & $0.614 \pm 0.066[\mathrm{~Hz}]$ \\
Regression Bax slope & $0.668 \pm 0.070[\mathrm{~Hz}]$ & $0.808 \pm 0.052[\mathrm{~Hz}]$ & $0.817 \pm 0.052[\mathrm{~Hz}]$ \\
\hline
\end{tabular}

Contrary, removal of the four residues in the N-terminal $\alpha$-helix (T6, D9, A14, R15; red diamonds in Figure 19B) changed the quality of the fit only slightly (Table 6). Thus, the data suggest that the $\alpha$-helix structure in the mVDAC1 crystal is at least similar to the structure of hVDAC1 in solution. However, the quality of the data might not be sufficient to exclude other conformations of the helix.

The alignment tensor resulting from the fit of 64 residues (including the $\alpha$-helix residues) was oriented with the z-axis directed through the pore of the channel (Figure 19C), coinciding with the short axis of the gel pores in a gel compressed along the same axis (the magnetic field).

In conclusion, RDC values with medium quality can be recorded from hVDAC1 in LDAO and partially aligned in positively charged polyacrylamide gels. In order to use the RDC values for structure refinement and determination of the $\alpha$-helix orientation in solution under various conditions, further optimization is required. This could include: (i) gels (e.g. reduction of the initial gel concentration to 5 or $6 \%$ (Cierpicki and Bushweller 2004) or reduced amount of cross-linking reagent), (ii) protein sample (concentration, perdeuteration, stabilizing mutations - e.g. E73V), and (iii) pulse sequences (e.g. 3D scaled $\left({ }^{1} \mathrm{~J}_{\mathrm{NH}}+{ }^{1} \mathrm{D}_{\mathrm{NH}}\right)$ spectra (Arbogast et al. 2010; Kontaxis et al. 2000; Yang et al. 1999)). 


\subsection{Dynamics of hVDAC1}

\subsubsection{Conformational instability of the N-terminal part of hVDAC1 probed by}

\section{H/D exchange}

As mentioned above (section 3.2.1), signal intensities in the $\mathrm{N}$-terminal $\beta$-barrel region of $w t \mathrm{hVDAC1}$ were lower than in E73V hVDAC1, such that seven residues in $\beta 4$ and $\beta 5$ could not be assigned in $w t$ hVDAC1 (Figure 12). Furthermore, low signal intensities complicated the resonance assignment in the second part of the $\mathrm{N}$-terminal $\alpha$ helix/linker. In order to investigate the conformational stability of the $\beta$-barrel and the $\alpha$-helix, we performed amide proton hydrogen/deuterium (H/D) exchange of $w t$ and E73V hVDAC1 ${ }^{3}$. Hydrogen-bonds are the main cause for slowed H/D exchange and indicate the presence of secondary structure (Englander and Kallenbach 1983; Krishna et al. 2004). $\beta$-barrel residues are usually stabilized by strong inter-strand hydrogenbonds that resist amide proton exchange for hours or days. In $\alpha$-helices the $\mathrm{N}$-terminal four residues exchange fast due to the absence of hydrogen bond acceptors for amides in the first helical turn (Wagner et al. 1994).

For $\mathrm{H} / \mathrm{D}$ exchange, hVDAC1 was solubilized in $100 \% \mathrm{D}_{2} \mathrm{O}$ and peak intensitiy changes were followed. Surprisingly, in $w t$ hVDAC1 residues in the N-terminal $\beta$ strands $\beta 1-\beta 3$ exchanged rapidly, such that many resonances were already broadened in the first spectrum after resolubilisation (Figure 20A). This demonstrates that hydrogen bonds in the N-terminal barrel region are transiently broken and suggests that the Nterminal $\beta$-strands undergo conformational exchange. In contrast, resonances in $\beta 4-\beta 5$ (as far as assigned) and $\beta 6-\beta 19$ showed protection from H/D exchange except for the edges of $\beta$-strands that exchanged fast in all $\beta$-strands (Figure 20).

\footnotetext{
${ }^{3}$ The entire section 3.3.1 is reproduced from our publication (Bayrhuber et al. 2008) with the improved backbone resonance assignment and contains some identical text passages.
} 

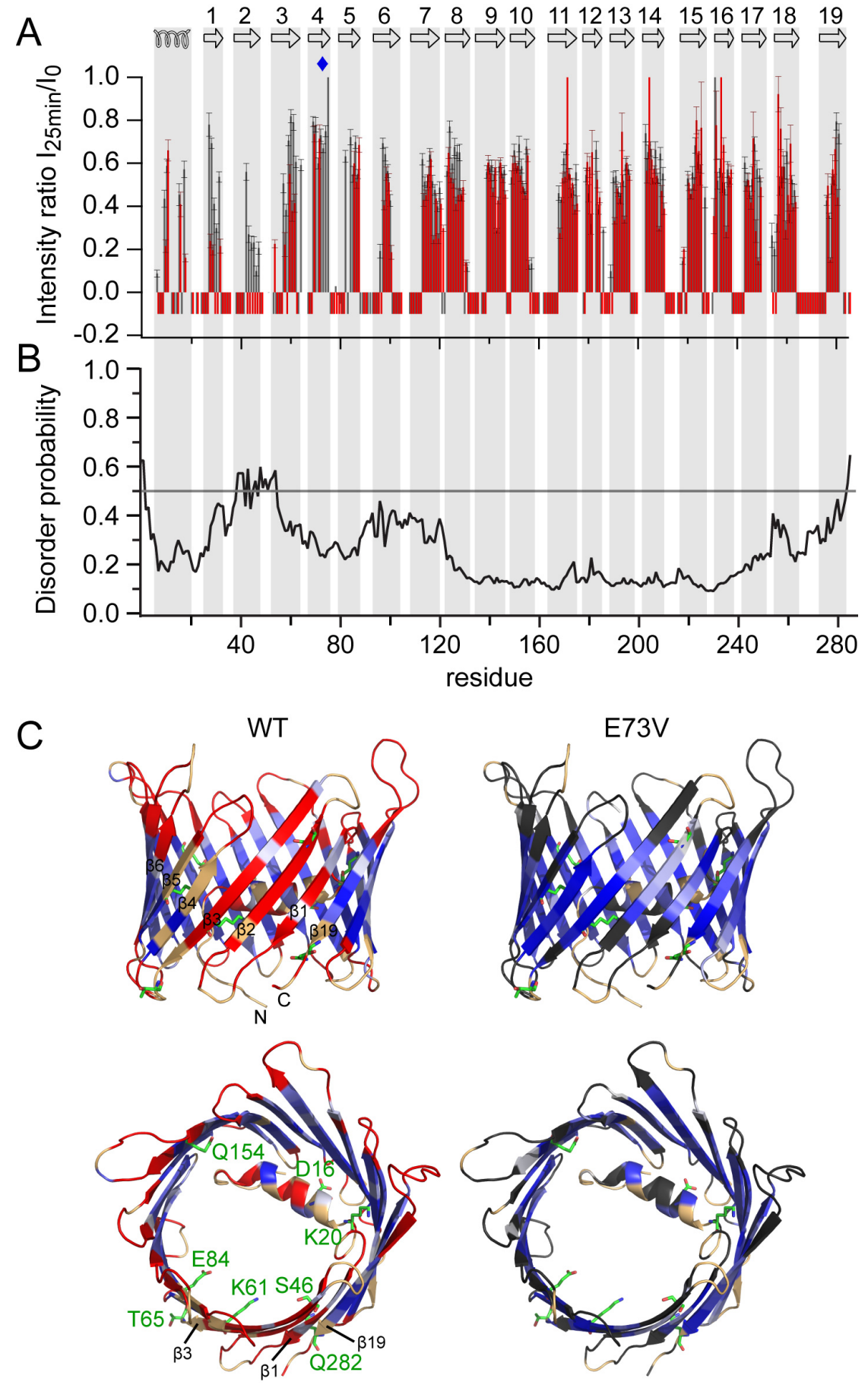

Figure 20. Conformational instability of the N-terminal region of the hVDAC1 barrel. (A) Comparison of amide proton exchange in $w t$ (red) and E73V (grey) hVDAC1 at $37{ }^{\circ} \mathrm{C}, \mathrm{pH}$ 6.8. Shown are signal intensity ratios of hVDAC1 in $\mathrm{D}_{2} \mathrm{O}$ versus $\mathrm{H}_{2} \mathrm{O}$ ( \pm standard error), completed two hours after transfer into $100 \% \mathrm{D}_{2} \mathrm{O}$. For clarity, resonances broadened beyond detection are set to a value of -0.1 and the scale was cut above 1.0. D16 was weak in reference spectra and omitted from the analysis. The location of the $19 \beta$-strands and the $\alpha$-helix are shown on top. The position of E73 is highlighted by a blue diamond. (B) Disorder probability in hVDAC1 as predicted by the DisProt web server (Sickmeier et al. 2007). The grey line indicates the threashold value above which residues are predicted as disordered. This data was provided by Monika Bayrhuber. 
Figure 20 (continued) (C) Ribbon representation of $w t$ hVDAC1 (left) and E73V hVDAC1 (right) in a side view and rotated by $90^{\circ}$ resulting in a top view. Signal intensity ratios from the H/D exchange were visualized by using a continuous white/blue scale (white, $<0.1$; blue, $>0.7$ ). Residues without detectable signal in the exchange ${ }^{1} \mathrm{H},{ }^{15} \mathrm{~N}$-TROSY are shown in red for $w t \mathrm{hVDAC} 1$ and grey for E73V hVDAC1. Unassigned residues are coloured in light yellow. Residues that affect the voltage gating of scVDAC (D16, K20, K46, K61, K65, K84, Q154, Q282) (Thomas et al. 1993) are shown with side chains. The figure was modified from (Bayrhuber et al. 2008) with the increased backbone resonance assignment.

Furthermore, the H/D exchange revealed that the solvent exchange of many $\alpha$ helical residues (T6, Y7, A8, D9, K12, A14, and V17) is much faster than in the Cterminal part of the hVDAC1 barrel (Figure 20). V17 is located in a crowded region and might also be completely exchanged. D16 was weak in the reference spectrum, but most likely exchanged. As pointed out above, the exchange of the four N-terminal $\alpha$-helical residues (T6-D9, Figure 20) is expected. Due to the kink around residues 9-11 (Figure 13), residues 12-15 are expected to exchange fast as well. In contrast, the fast exchange in residues $\mathrm{V} 17$ and $\mathrm{K} 20$ suggests that the conformation of the second helical part is destabilized or has a pronounced solvent accessibility.

In line with the conformational exchange in the N-terminal strands $\beta 1-\beta 3$, computational analysis based on the primary sequence of hVDAC1 predicted a higher disorder propensity for the same $\beta$-strands (Figure 20B). Mutations that affected the voltage sensitivity of Saccharomyces cerevisiae VDAC (scVDAC) are mainly located in the same region (D16 in the $\alpha$-helix, K20 in the linker, and K46, K61, K65, and K84 in $\beta$-strands $\beta 1-\beta 5$ (Figure 20C) (Thomas et al. 1993)).

The conformational stability of hVDAC1 is particularly influenced by the glutamic acid residue 73. E73 is conserved in VDAC2 and VDAC from mammals and some fungi (Supporting figure 3). Its side chain points to the hydrophobic environment, disrupting the amphipathic pattern in $\beta 4$. Mutation of E73 to valine strongly reduced the $\mathrm{H} / \mathrm{D}$ exchange rates in the $\mathrm{N}$-terminal three $\beta$-strands (Figure 20A, C), suggesting that the conformational flexibility in this region is reduced by the mutation. In addition, the exchange of V17 is most likely reduced in E73V hVDAC1. 


\subsubsection{Backbone dynamics of hVDAC1}

Next we characterized the backbone dynamics of hVDAC1 in more detail. ${ }^{4}$ The fast $\mathrm{H} / \mathrm{D}$ exchange in the $\mathrm{N}$-terminal barrel region points to conformational exchange on a time scale of minutes or faster (Figure 20). In addition, average peak intensities observed in ${ }^{1} \mathrm{H},{ }^{15} \mathrm{~N}$-TROSY spectra for residues located in the N-terminal six $\beta$-strands were about 1.6-fold lower than those of the central $\beta$-strands $(\beta 7-\beta 14)$ (Figure 21).
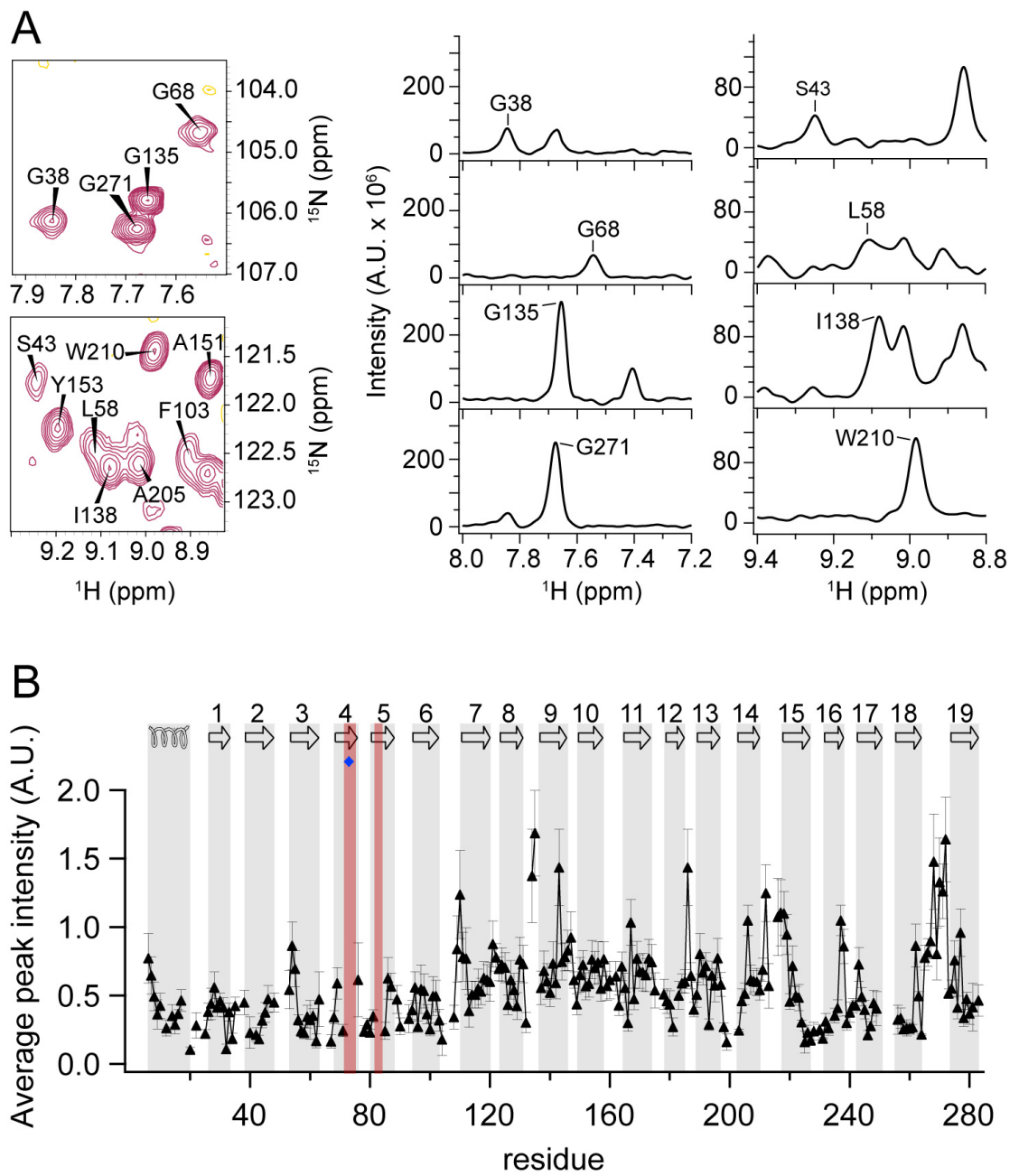

Figure 21. Signals in the N-terminal $\boldsymbol{\beta}$-strands and the helix of VDAC1 are broadened. (A) Enlarged spectral regions and 1D traces of a ${ }^{1} \mathrm{H},{ }^{15} \mathrm{~N}$-TROSY spectrum of hVDAC1 measured at a ${ }^{1} \mathrm{H}$ frequency of $900 \mathrm{MHz}$ showing examples of broadened and non-broadened peaks. (B) Average peak intensities (mean \pm standard error of the mean, $\mathrm{n}=3$ ) of $w t$ VDAC1 from $2 \mathrm{D}{ }^{1} \mathrm{H},{ }^{15} \mathrm{~N}$-spectra measured at 900 or $800 \mathrm{MHz}$. The topology of VDAC1 is indicated on top, with secondary structure elements highlighted in grey. Residues in the N-terminal $\beta$-strands that could not be assigned in $w t$ hVDAC1 are highlighted in red; E73 is indicated by a blue diamond.

\footnotetext{
${ }^{4}$ The full sections 3.3.2 to 3.3.4 are part of our recent publication (Villinger et al. 2010) and contain mainly identical text passages.
} 
Broadening was also observed in the N-terminal $\alpha$-helix and the linker, continuously decreasing from T6 to G25, as well as in the C-terminal $\beta$-strands $\beta 15-19$ (Figure 21). However, continuing resonance broadening of residues in $\beta 15-\beta 17$ with increasing sample age advices caution when the dynamics in this region are examined. In contrast, lower signal intensities in the $\mathrm{N}$-terminal six $\beta$-strands, $\beta 19$, and the second $\alpha$-helical part were observed consistently for many samples with different age. NMR signal broadening can be a consequence of either increased rigidity in the ps-ns range or increased conformational exchange that is intermediate on the NMR time scale ( $\mu$ s-ms dynamics).

To probe for the influence of oligomerization on the observed signal broadening we measured a $2 \mathrm{D}{ }^{1} \mathrm{H},{ }^{15} \mathrm{~N}$-TROSY spectrum at a five-fold lower concentration. Despite the five-fold lower concentration the strong signal broadening in the $\mathrm{N}$-terminal $\beta$ strands and the $\alpha$-helix/linker remained (Figure 22). In addition, the N-terminal three $\beta$ strands of $w t$ hVDAC1 exhibit fast H/D exchange (Figure 20), whereas oligomerization would be expected to cause lower H/D exchange rates in the interaction site. Thus, oligomerization is not a major factor responsible for the observed signal broadening in the N-terminal $\beta$-strands or the $\alpha$-helix of hVDAC1.

Further contributions to signal broadening may come from remote influences caused by motions of nearby groups (side chains or detergent molecules), rather than by motions of the respective amide groups themselves. Again, these influences do not explain the fast H/D exchange (Figure 20).

To determine the time scale of dynamics causing the NMR signal broadening in the N-terminus of hVDAC1, we measured heteronuclear NOE (HetNOE) values (Zhu et al. 2000) and ${ }^{15} \mathrm{~N}$ transverse spin relaxation rates (Wang et al. 2003). HetNOE values, which have a field-dependent maximum value of approximately $0.7-0.9$ for a fully rigid backbone, are sensitive to dynamics on the ps-ns time scale while ${ }^{15} \mathrm{~N}$ transverse spin relaxation rates probe chemical exchange occurring in the $\mu \mathrm{s}-\mathrm{ms}$ range. As expected, lower HetNOE values, indicative for increased flexibility on the ps-ns time scale, were observed at the rims of $\beta$-strands and in the short turns and long loops (Figure 23). 
A
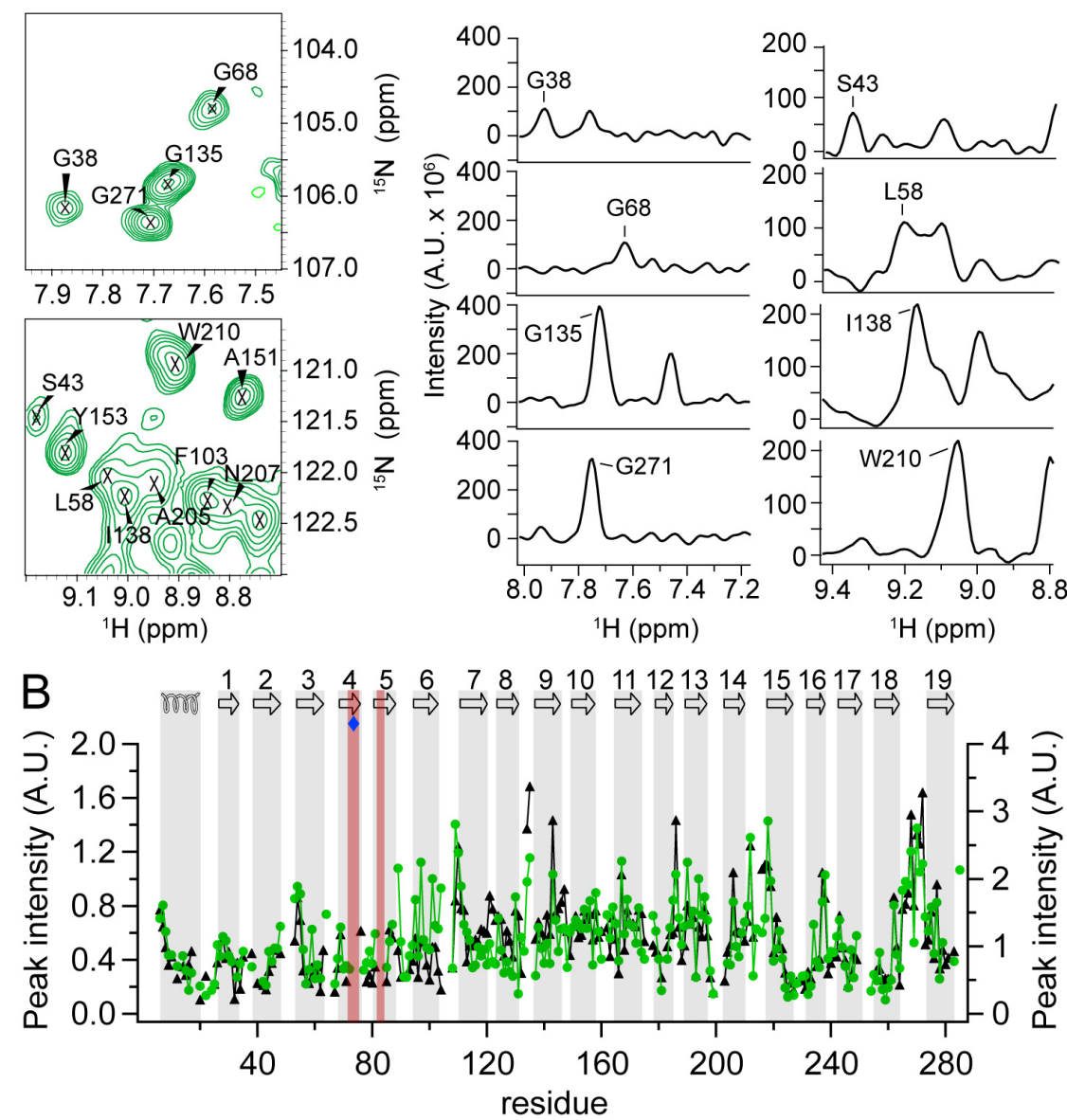

Figure 22. The strong signal broadening in the $\mathrm{N}$-terminus remains upon dilution of hVDAC1. (A) Enlarged spectral regions and 1D traces of a ${ }^{1} \mathrm{H},{ }^{15} \mathrm{~N}-\mathrm{TROSY}$ spectrum of hVDAC1 measured at a ${ }^{1} \mathrm{H}$ frequency of $900 \mathrm{MHz}$ and a protein concentration of $0.1 \mathrm{mM}$ showing the same resonances as in Figure 21A. (B) Average peak intensities of $w t$ VDAC1 at a concentration of approximately $0.5 \mathrm{mM}$ (black) as shown in Figure 21B and peak intensities of $w t$ hVDAC1 at a concentration of $0.1 \mathrm{mM}$ (green) from 2D ${ }^{1} \mathrm{H},{ }^{15} \mathrm{~N}$-TROSY spectra measured at $900 \mathrm{MHz}$. Errors in peak intensities of $0.1 \mathrm{mM}$ VDAC are smaller than the markers. For clarity, standard errors of the high concentration sample (black) are omitted. The topology of VDAC1 is indicated on top, with secondary structure elements highlighted in grey.

Moreover, the HetNOE value of the first helical residue (T6) is high, while the values decrease slightly along the $\alpha$-helix and are reduced for some linker residues (K20, F24, G25). This shows that the rigidity decreases from the $\mathrm{N}$ - to the C-terminal part of the $\alpha$-helix and towards the linker. HetNOE values within different hVDAC1 $\beta$ strands were similar with an average value of 0.75 , while slightly smaller HetNOE values were observed for $\beta 2, \beta 3$ and $\beta 19$ (Figure 23). These values indicate a local spot of elevated ps-ns dynamics in the N-terminal barrel region, while the rest of the barrel is rather rigid on that time scale. 


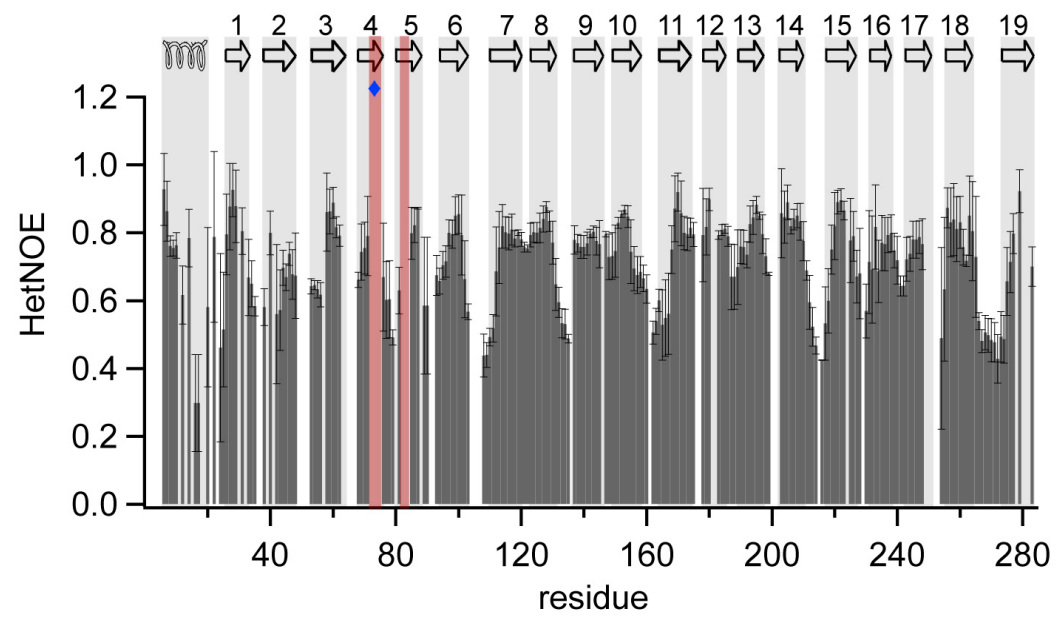

Figure 23. Rigidity of hVDAC1 $\boldsymbol{\beta}$-strands on the ps-ns time scale. Heteronuclear NOE values (HetNOE) of hVDAC1 measured at $800 \mathrm{MHz}$. Intensity ratios were averaged over a three-residue window.

Residues with increased flexibility on the ps-ns time scale are expected to result in more intense NMR signals, in contrast to what was observed for the $\mathrm{N}$-terminal $\beta$ strands and the second $\alpha$-helical part in hVDAC1 (Figure 21). Thus, the combination of NMR signal intensities and HetNOE measurements suggests the presence of chemical exchange that is intermediate on the NMR time scale.

Therefore, we directly measured chemical exchange contributions to ${ }^{15} \mathrm{~N}$ spin relaxation rates $\left(\mathrm{R}_{\mathrm{ex}}\right)$ (Wang et al. 2003). Although $\mathrm{R}_{\mathrm{ex}}$ values were distributed around $\sim 0 \mathrm{~s}^{-1}$ with a rather large standard deviation due to variation in magnitude and orientation of the ${ }^{15} \mathrm{~N}$ CSA tensor (Fushman et al. 1998), several regions of elevated exchange were determined (Figure 24A). Residues A14, R15, and D16 in the second part of the $\alpha$-helix and Y22 in the linker showed higher exchange rates than residues in the first part of the helix (T6-L10) (Figure 24A). In addition, residues located in $\beta 2-\beta 7$, $\beta 16$, and $\beta 19$ showed increased chemical exchange rates with average values $\geq 3.5 \mathrm{~s}^{-1}$ (Figure 24). $\beta 19$ exhibited increased values especially in the first half of the strand, which might be attributed to a mismatch in hydrogen bonding to the shorter strand $\beta 1$. Furthermore, the parallel alignment of $\beta 1$ and $\beta 19$ might result in a lower intrinsic stability and thus favour the conformational exchange observed in $\beta 19$. 

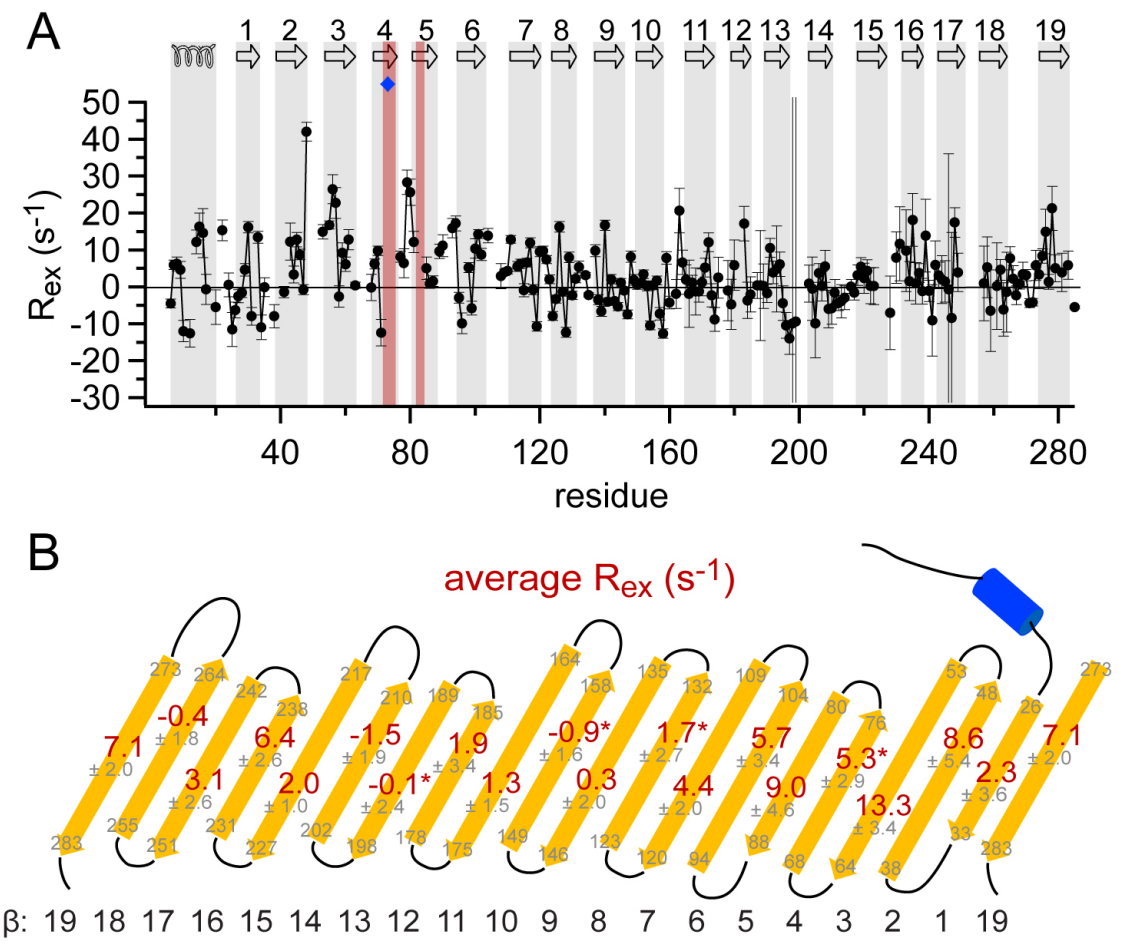

Figure 24. $\mu \mathrm{s}-\mathrm{ms}$ dynamics are significantly increased in the N-terminus of hVDAC1. (A) Chemical exchange rates $\left(\mathrm{R}_{\mathrm{ex}}\right)$ measured at $900 \mathrm{MHz}$ (value \pm standard error). The topology of VDAC1 is indicated on top, with secondary structure elements highlighted in grey. Residues that could not be assigned are highlighted in red; E73 is indicated by a blue diamond. (B) Topology map of hVDAC1 showing average $R_{e x}$ values for all $\beta$-strands in red ( \pm standard error, grey). $\beta$-strands with first and last residue (grey) are indicated as yellow arrows, the $\mathrm{N}$-terminal helix as a blue cylinder. Average $\mathrm{R}_{\mathrm{ex}}$ values obtained after removal of negative outliers with values $<-12$ are marked with an asterisk.

The most pronounced chemical exchange was present in the $\mathrm{N}$-terminal barrel region ranging from $\beta 2$ to $\beta 7$ with the largest average $R_{e x}$ values seen in $\beta 3$ for K53 $\left(14.9 \mathrm{~s}^{-1}\right)$, T55 $\left(16.7 \mathrm{~s}^{-1}\right)$, G56 $\left(26.7 \mathrm{~s}^{-1}\right)$, S57 $\left(22.7 \mathrm{~s}^{-1}\right)$, T60 $\left(6.15 \mathrm{~s}^{-1}\right)$ and K61 $\left(12.8 \mathrm{~s}^{-1}\right)$. In addition to the seven residues in $\beta 4-\beta 5$ that could not be assigned (Figure 12 and Figure 24), several residues in the N-terminal barrel region (e.g. K34, T42, S43, G45, and S57) showed two distinct resonances in $2 \mathrm{D}{ }^{1} \mathrm{H},{ }^{15} \mathrm{~N}-\mathrm{TROSY}$ spectra. In the case of S57, two resonances were also observed in 3D TROSY-HNCA spectra (Figure 25), indicating the existence of a second conformation in slow exchange. Although no second set of resonances was identified in the helix, such a set was indicated for the linker region (see section 3.2.2). Thus, pronounced $\mu$ s-ms conformational exchange is present in the N-terminal part of the hVDAC1 barrel centred at strands $\beta 3-\beta 4$ and in the second part of the $\mathrm{N}$-terminal $\alpha$-helix and the linker. 


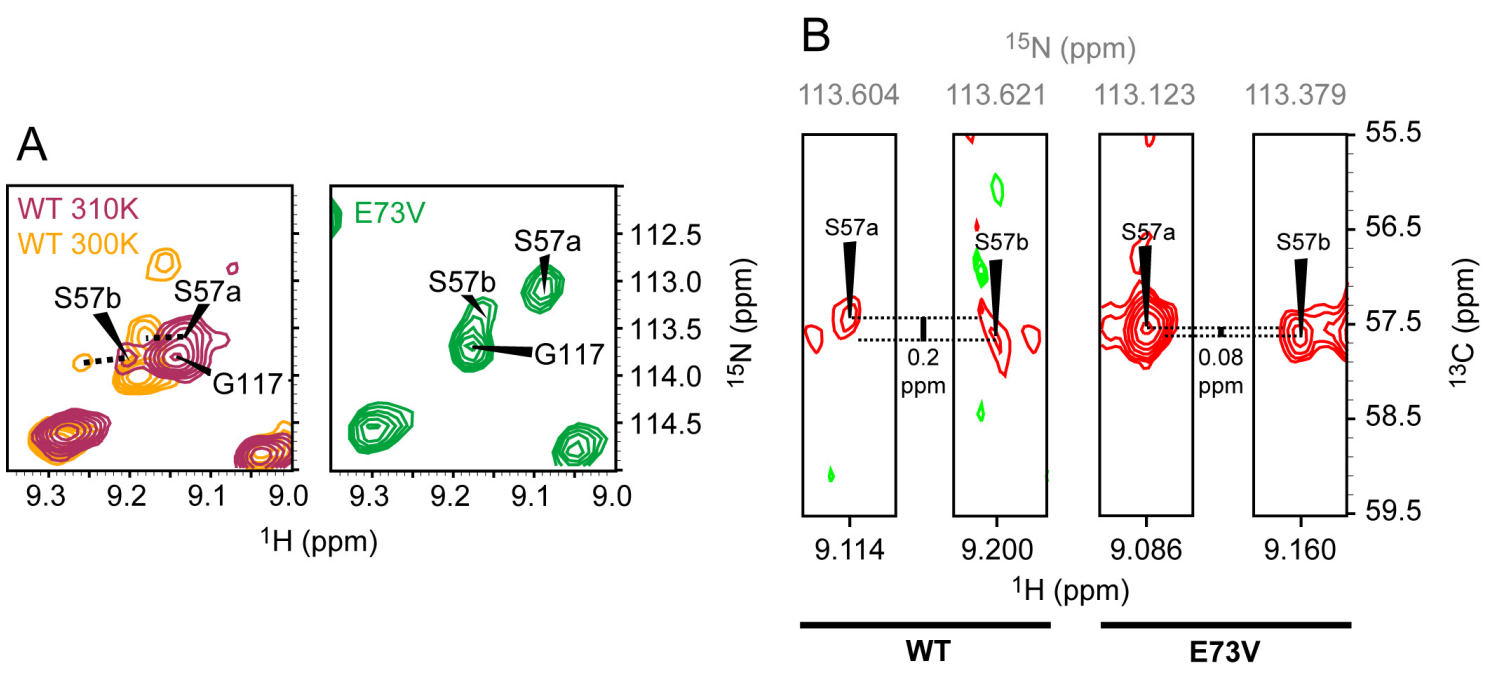

Figure 25. Chemical exchange of residue S57. (A) Enlarged regions of ${ }^{1} \mathrm{H},{ }^{15} \mathrm{~N}$-TROSY spectra of $w t$ and E73V hVDAC1 measured at a ${ }^{1} \mathrm{H}$ frequency of $900 \mathrm{MHz}$ showing the two resonances of S57 (S57a and S57b). The dashed lines indicate the temperature-dependent shift of both resonances. (B) $2 \mathrm{D}{ }^{1} \mathrm{H},{ }^{13} \mathrm{C}$ strips from 3D TROSY-HNCA spectra of $w t$ and E73V hVDAC1 measured at a ${ }^{1} \mathrm{H}$ frequency of $900 \mathrm{MHz}$ showing the S57 resonances. The dashed lines indicate the differences in $\mathrm{C} \alpha$ chemical shift.

\subsubsection{Increased B-factors in the crystal structure of VDAC correlate with low-}

\section{frequency modes derived from Gaussian network model analysis}

Dynamic features of proteins are also present in crystals, partly captured by DebyeWaller factors or B-factors. B-factors describe the positional uncertainty of atoms and measure both thermal motions (dynamic disorder) and small differences in the structure of individual molecules (static disorder) (Ringe and Petsko 1986). VDAC1 from mouse was crystallized in a bicellar environment (Ujwal et al. 2008). mVDAC1 differs from human VDAC1 by just four amino acid substitutions, namely T55N, M129V, A160S and $1227 \mathrm{~V}$, and the 3D structures of human and murine VDAC1 are almost identical (rmsd value of $1.45 \AA$ for $\mathrm{C} \alpha$ atoms of residues 26-283) (Bayrhuber et al. 2008; Ujwal et al. 2008). In the mVDAC1 crystal structure B-factors are highly non-uniform (Figure $26 \mathrm{~A})$. Whereas in the central strands average B-factors of $41.3 \AA^{2}$ were observed, $\beta 2-\beta 7$ and $\beta 18-\beta 19$ showed average values of $50.6 \AA^{2}$ and $48.9 \AA^{2}$, respectively. Low Bfactors also occurred in the complete $\alpha$-helix and linker, averaging to $40.1 \AA^{2}$ and $43.1 \AA^{2}$, respectively.

Crystallographic B-factors can be predicted from a 3D structure by a diverse range of computational methods. 

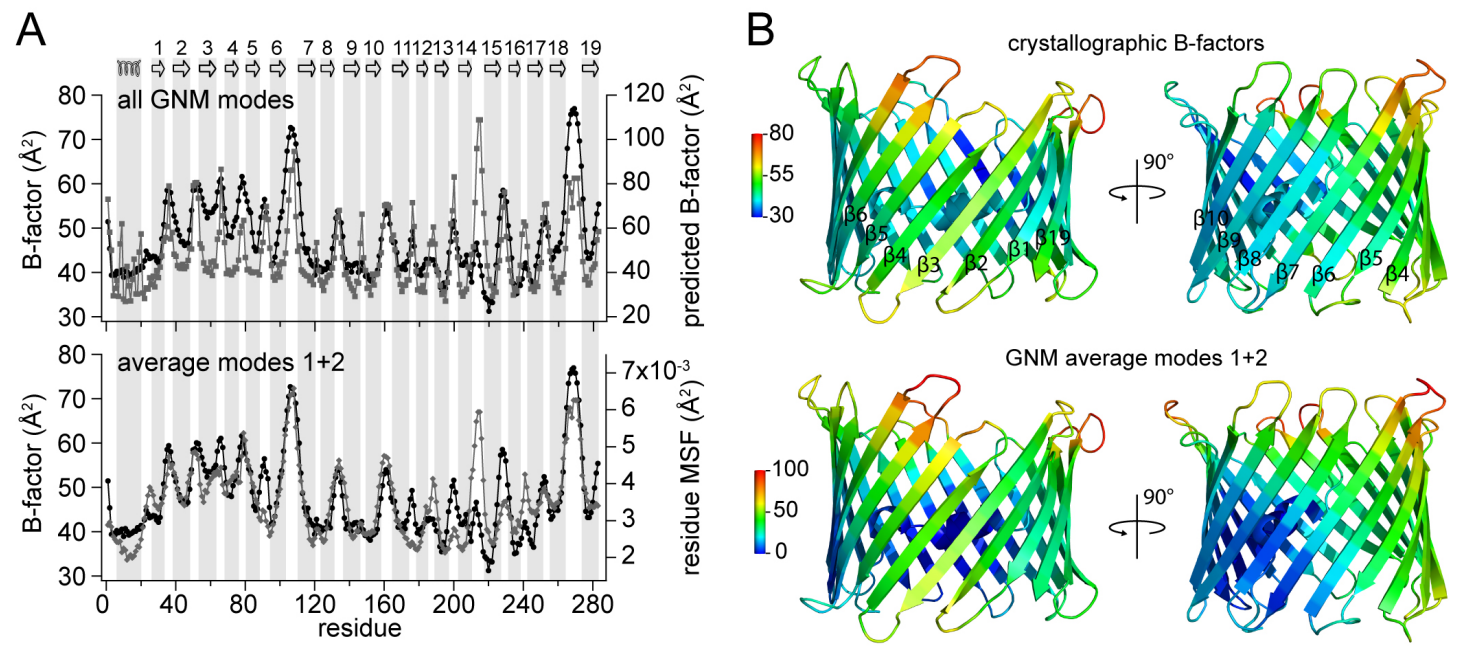

Figure 26. Intrinsic flexibility of the mVDAC1. (A) Crystallographic B-factor $(\bullet)$, predicted B-factors for all modeds ( $\square$, upper panel), and mobility predicted from the two lowest frequency modes $(\diamond$, lower panel), respectively. The topology of VDAC1 is indicated on top, with secondary structure elements highlighted in grey. (B) Cartoon representations of mVDAC1 generated from the crystal structure (PDB code: $3 \mathrm{EMN}$ ), colour-coded by crystallographic B-factors and GNM predicted residue fluctuations for the average of the two lowest frequency modes, rescaled by the oGNM server (Yang et al. 2006) to yield effective B-factors.

We used the Gaussian network model (GNM) (Yang et al. 2006), an isotropic, $\mathrm{C} \alpha$-atom connected elastic network model, to test if the unusually high B-factors of the $\mathrm{N}$-terminal $\beta$-strands are related to the intrinsic stability of the 3D structure. While Bfactors predicted from all modes in the GNM analysis yielded low correlations with crystallographic B-factors, the use of the average of the two lowest frequency modes resulted in a notable agreement between crystallographic B-factors and GNM-predicted mobility (Figure 26A). The correlation coefficient $\left(\mathrm{R}^{2}\right)$ was 0.61 overall and 0.58 for $\beta$ strands only, which is substantial regarding the complexity of the prediction. The exact frequencies cannot be extracted from the GNM analysis without knowledge of the network force constant. However, motions with low frequency are believed to be associated with high cooperativity, suggesting that the high crystallographic B-factors and GNM-predicted low-frequency fluctuations show a predisposition of the N-terminal barrel region for its involvement in collective motions. Mapping of B-factors and GNMderived residue fluctuations onto the crystal structure showed that $\beta$-strands next to the $\alpha$-helix (the central strands $\beta 8-\beta 15$ ) and the complete $\alpha$-helix were highly ordered, ascribing a stabilizing function to the N-terminal $\alpha$-helix (residues 6-20) (Figure 26B). Such a stabilizing role of the $\alpha$-helix has also been suggested on the basis of solid-state 
NMR results on an N-terminally truncated version of hVDAC1 (Schneider et al. 2010). In contrast, high flexibility was found from $\beta 2$ to the top end of $\beta 7$ (Figure 26B).

Thus, the same barrel regions that are strongly affected by chemical exchange on the $\mu \mathrm{s}-\mathrm{ms}$ time scale have an increased disorder in the crystal structure, in agreement with a lower intrinsic stability predicted from the two lowest eigenmodes of the GNM analysis. In contrast, B-factors and GNM analysis disagree with the elevated NMRderived $\mu$ s-ms chemical exchange, ps-ns flexibility, and low signal intensities observed for the second part of the $\alpha$-helix and indicate a rigid $\alpha$-helical conformation.

\subsubsection{The single-point mutation E73V and the reaction of E73 with DCCD stabilize the hVDAC1 barrel}

Functional studies have ascribed an important function to E73, showing that ruthenium red and hexokinase-I mediated gating and apoptosis inhibition are impaired in E73Q VDAC (Zaid et al. 2005). Moreover, we showed that E73V hVDAC1 exhibits reduced $\mathrm{H} / \mathrm{D}$-exchange in the $\mathrm{N}$-terminal barrel region (Figure 20), indicating that motions on a second to minute time scale responsible for H/D exchange are strongly quenched. To dissect the influence of E73 on hVDAC1 dynamics in solution, we analyzed NMR signal intensities for the single point-mutation E73V. Strikingly, NMR signal intensities were strongly increased (by a factor of $\sim 1.64$ on average) in the $\mathrm{N}$-terminus in E73V hVDAC1 compared to the wild-type protein (Figure 27A). The changes were centred on E73 involving a large area of the barrel (Figure 27A). In addition, signal intensities from residues in the linker (K20 and Y22) increased strongly, and residues in the Nterminal helix (D9, L10, A14, R15) exhibited slightly higher signal intensities than the central strands $(\beta 9-\beta 12)$.

In case of $w t$ hVDAC1 the peak broadening was shown to be caused by chemical exchange (Figure 24), indicating that the mutation E73V strongly reduces the $\mu \mathrm{s}-\mathrm{ms}$ time scale dynamics in the N-terminal barrel region. Indeed, a comparison of relaxation rates of E73V hVDAC1 with $w t$ values (Figure 27B) demonstrates a reduction of $\mu \mathrm{s}-\mathrm{ms}$ time scale dynamics for $\beta 1-\beta 9$ and $\beta 19$, with the largest changes in $\beta 3$ and $\beta 5$ as well as the loop connecting $\beta 4$ and $\beta 5$. 

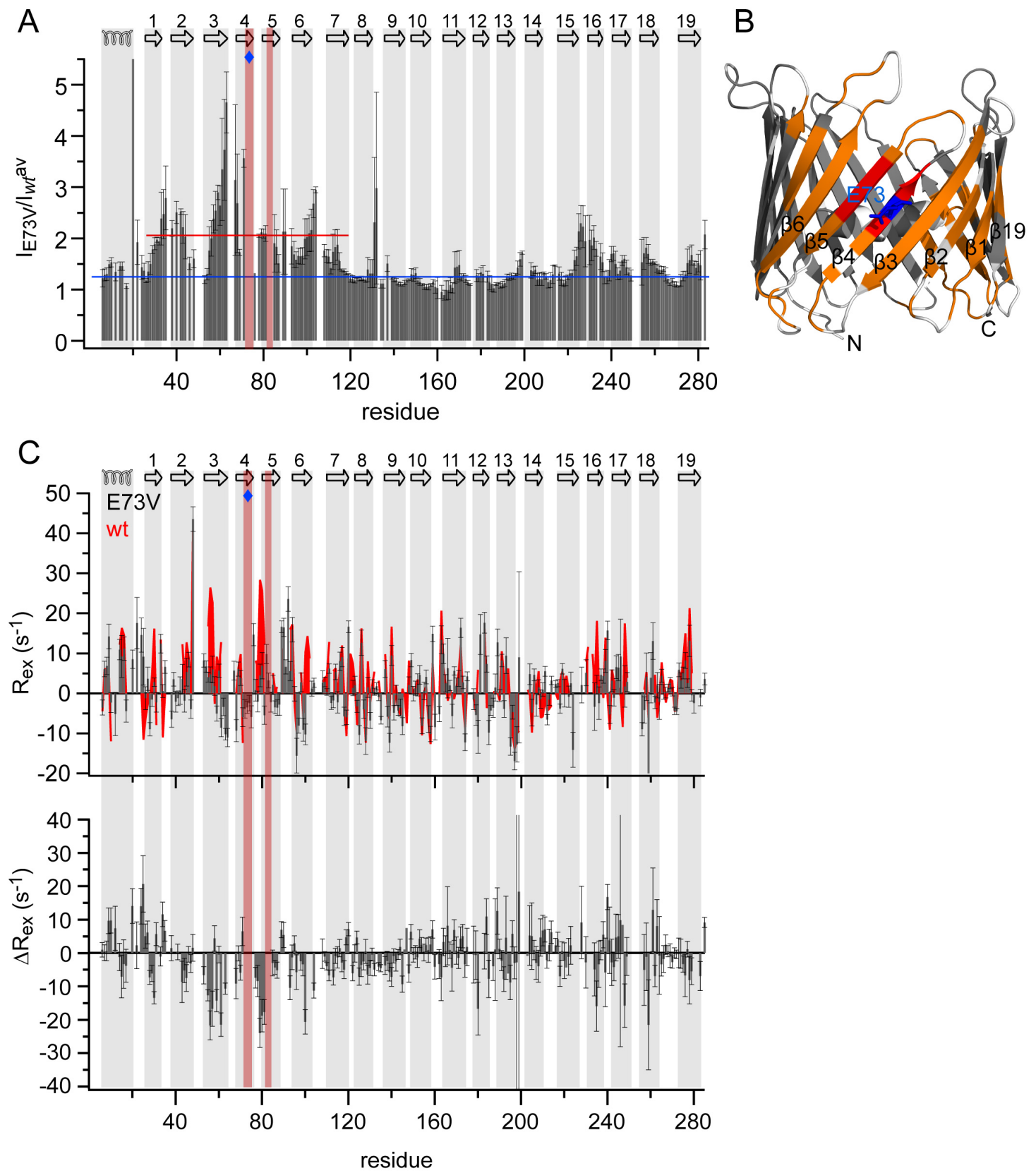

Figure 27. Stabilization of hVDAC1 by the E73V mutation (A) Ratio of peak intensities between E73V and $w t$ hVDAC1 observed in ${ }^{1} \mathrm{H},{ }^{15} \mathrm{~N}$-spectra, averaged over a three-residue window $( \pm$ standard error). The blue line indicates the average value for residues $120-217$. The red line indicates the average value for residues 26-119 ( $\beta 1-\beta 7)$. (B) Residues with an intensity ratio above 1.5 were mapped onto the 3D structure of hVDAC1 (PDB code: 2JK4) in orange. Residues that, in contrast to E73V hVDAC1, could not be assigned in $w t$ hVDAC1 are mapped in red. (C) Top: chemical exchange rates $\left(\mathrm{R}_{\mathrm{ex}}\right)$ of E73V (grey bars, \pm standard error) compared to $w t$ values from Figure 24A (red area, for clarity errors were omitted). Difference values $(\mathrm{E} 73 \mathrm{~V}-w t)$ are shown below ( \pm standard error). The topology of VDAC1 is indicated on top, with secondary structure elements highlighted in grey. Residues that could not be assigned in $w t$ hVDAC1 are highlighted in red; E73 is indicated by a blue diamond.

Additionally, exchange rates in the second part of the $\alpha$-helix (A14, R15, and D16) decreased slightly, although the changes were within standard errors. Curiously, 
exchange rates of the residues K20, Y22, F24, and G25 increased further by the mutation, in contrast to the increased intensity ratios (Figure 21).

E73 can also be modified by reaction with N,N'-dicyclohexylcarbodiimide (DCCD), a chemical that reacts with aspartic and glutamic acid residues in a hydrophobic surrounding. DCCD has previously been shown to specifically bind VDAC1 in mitochondrial membranes at position E73 (De Pinto et al. 1993). The specificity of the reaction is also given in micellar solution, since chemical shift changes upon DCCD addition were localized close to E73 (Figure 28A).
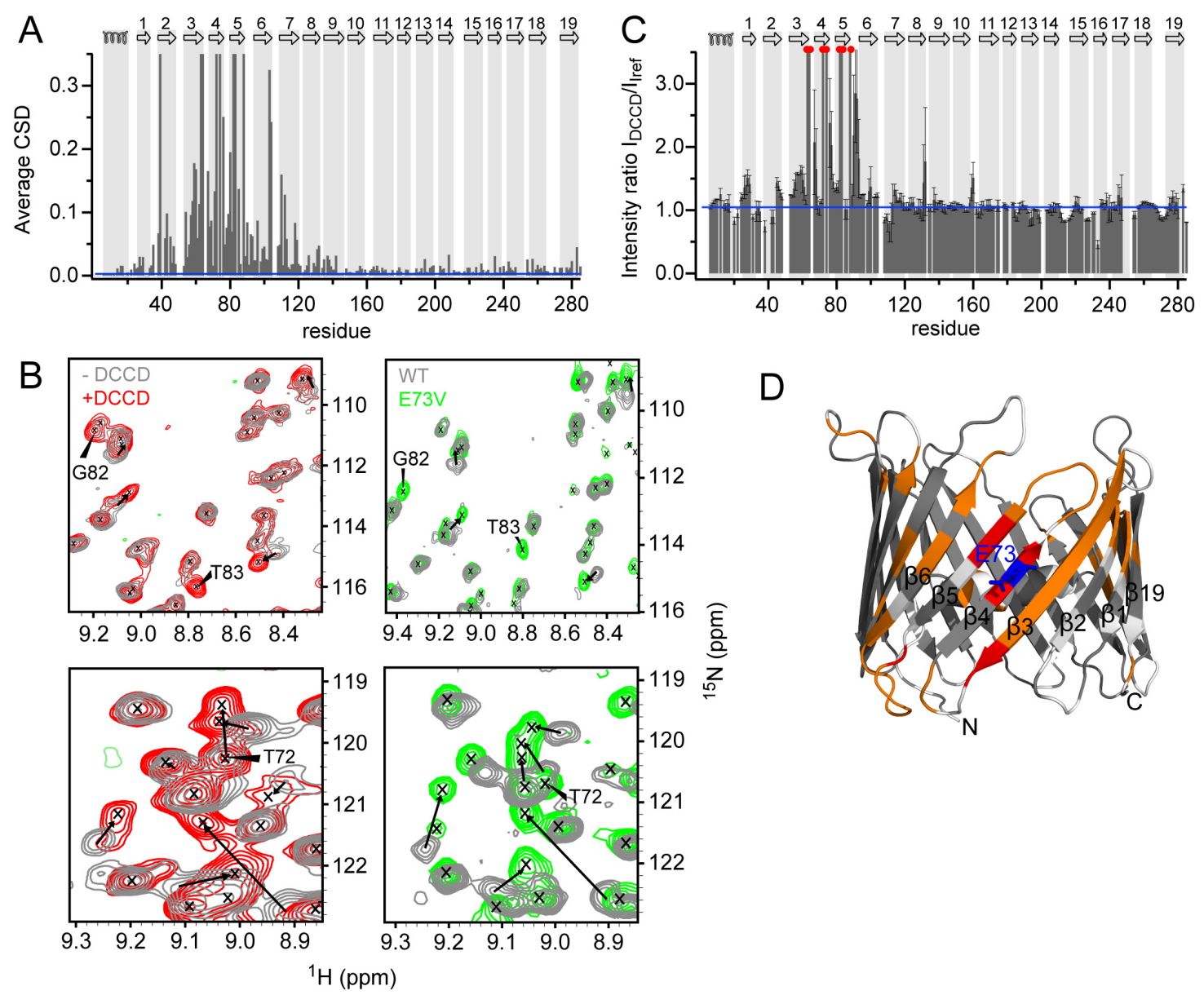

Figure 28. Stabilization of hVDAC1 by chemical modification with DCCD. (A) Average CSD from ${ }^{1} \mathrm{H},{ }^{15} \mathrm{~N}$-TROSY spectra upon reaction with DCCD. The average CSD of non-shifting residues is indicated by a blue line. (B) Enlarged spectral regions of ${ }^{1} \mathrm{H},{ }^{15} \mathrm{~N}$-TROSY spectra before and after incubation with DCCD (left) and of $w t$ hVDAC1 and E73V (right). Shifting peaks are indicated by arrows, newly appearing or strongly increased peaks are labelled with assignments. (C) Ratio of peak intensities between DCCD-bound and free hVDAC1 observed in ${ }^{1} \mathrm{H},{ }^{15} \mathrm{~N}$-TROSY spectra, averaged over a three-residue window ( \pm standard error). The blue line indicates the average intensity ratio for residues $117-285$. Newly appearing peaks are indicated by red circles. Residues with intensity ratios above 1.15 were mapped onto the structure of hVDAC1 (PDB code: 2JK4) in orange (D). Residues that appeared after DCCD reaction are mapped in red. 
The shifts were similar to chemical shifts in E73V hVDAC1 spectra (Figure 28B). Importantly, reaction with DCCD resulted in a similar increase in peak intensities as was the case for E73V (by a factor of $\sim 1.46$ for DCCD, compared to $\sim 1.64$ for $\mathrm{E} 73 \mathrm{~V}$ ) for many residues in the N-terminal part of the $\beta$-barrel (Figure 28C, D). In addition, most of the amide resonances that are missing in the $w t$ hVDAC1 spectrum but are present in E73V hVDAC1 spectra, appeared, e.g. R63, W64, T72, K74, G82, T83 and E88 (Figure 28B, C, D). No changes in NMR signal intensity were observed in the C-terminal region upon reaction with $\mathrm{DCCD}$, in contrast to the finding for E73V hVDAC1 (compare to Figure 27A). This could be related to differences in sample conditions (age dependent resonance broadening, detergent concentration) between $w t$ and E73V hVDAC1 samples.

\subsubsection{Reduced oligomerization of $\mathrm{hVDAC} 1$ is not the main cause for reduced dynamics in the $\mathrm{N}$-terminal barrel region}

To probe for the effect of E73 modification on oligomerization, we determined the rotational correlation times of hVDAC1 mutated and chemically modified at position E73 and compared it with the value of $w t$ hVDAC1 at a 6-fold reduced concentration. The rotational correlation time of $0.6 \mathrm{mM}$ hVDAC1 was $47 \mathrm{~ns}$ (Figure 29A, B), which is in agreement with the major presence of monomeric states in equilibrium with a small amount of dimers (see Materials and Methods). Mutation and chemical modification reduced the rotational correlation time by $7 \mathrm{~ns}$ (Figure 29A), indicating a smaller size by reduced dimerization. In addition, 6-fold dilution reduced the correlation time by the same amount (Figure 29B). However, one should note that effects other than dimerization can influence the rotational correlation time, for instance, changes in the micelle size and structure, due to variations in sample preparation or conditions (e.g. detergent concentration). Furthermore, the micelle structure might be influenced by the side chain of E73 and dynamics in the N-terminal barrel region. Dilution also induced an overall intensity increase by a factor of $\sim 1.28$ on average, with slightly larger intensity changes $(\sim 1.16$-fold relative to the overall intensity increase $)$ in the $\mathrm{N}$ terminal barrel region, including $\beta$-strands and loops in the region $\beta 2-\beta 5$, and loop L5-6 (Figure 29C). 

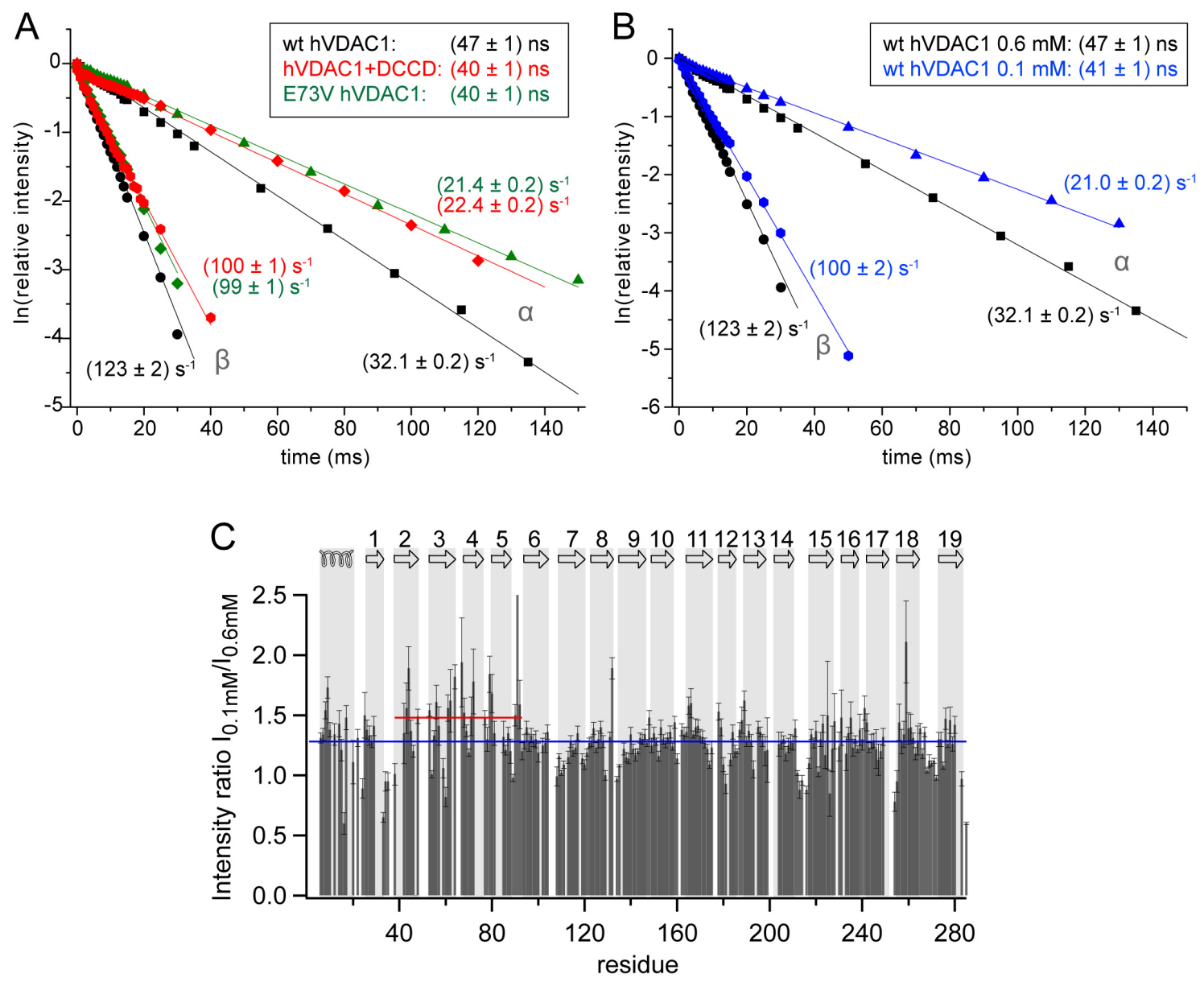

Figure 29. Influence of the state of E73 on VDAC oligomerization. TRACT experiment for (A) $\sim 0.6 \mathrm{mM}{ }^{2} \mathrm{H}(75 \%) /{ }^{15} \mathrm{~N}$ samples of $w t$ hVDAC1 (black), E73V hVDAC1 (green), and DCCD-modified hVDAC1 (red) and (B) $w t$ hVDAC1 at $0.6 \mathrm{mM}$ (black) and $0.1 \mathrm{mM}$ (blue) concentrations. Shown are the decays of the relative intensity of the ${ }^{1} \mathrm{H}$ NMR signal due to ${ }^{15} \mathrm{~N}$ relaxation of the slowly relaxing $\alpha$ and the rapidly relaxing $\beta$ component in a logarithmic scale. The rotational correlation times $\left(\tau_{\mathrm{C}}\right)$ are boxed. (C) Changes in peak intensity observed in $2 \mathrm{D}{ }^{1} \mathrm{H},{ }^{15} \mathrm{~N}$-TROSY spectra of hVDAC1 upon 6-fold dilution of $\mathrm{hVDAC1}$. The blue line indicates the average intensity ratio of 1.28 obtained after scaling according to protein concentration and number of scans recorded, the red line indicates the average value of 1.48 for residues 38-93.

The small increase (by a factor of 1.16) is not sufficient to explain the average intensity increase in the N-terminal barrel region by a factor of $\sim 1.64$ upon mutation or $\sim 1.46$ upon DCCD reaction of E73 (Figure 27 and Figure 28). Furthermore, dilution affected fewer residues in the N-terminus than modification of E73 and no new peaks appeared in contrast to modified hVDAC1 (compare to Figure 27 and Figure 28). This comparison shows that the majority of the reduction of $\mu \mathrm{s}-\mathrm{ms}$ dynamics in the $\mathrm{N}$ terminus is caused by a reduction in conformational exchange instead of changes in dimerization tendency. However, an overlap of the oligomerization region with the 
dynamic region is evident from the data, and a reduced dimerization for modified hVDAC1 might contribute to the observed reduction of $\mu \mathrm{s}-\mathrm{ms}$ dynamics.

\subsubsection{VDAC1 dynamics: Insight from molecular dynamics studies}

Bert de Groot and co-workers provided further insight into the dynamics of VDAC1 in a lipid bilayer and lipid interactions of mVDAC1 by MD simulation ${ }^{5}$. Since their work complements our NMR and GNM investigations, it is summarized in the following two sections.

\subsubsection{VDAC flexibility from MD simulations and the role of charge on E73}

Gaussian network model (GNM) analysis derives protein dynamics directly from the 3D structure by modelling the protein atoms (in this case only $\mathrm{C} \alpha$-atoms) within a defined distance range as harmonic springs (Bahar et al. 1997; Tirion 1996). However, the exact time scale of dynamics, the effect of point mutations and the influence of the membrane environment can not be investigated by the coarse-grained GNM analysis. Therefore, MD simulations of mVDAC1 inserted into a DMPC phospholipid bilayer were conducted to overcome these limitations. Since the charge status of E73 in solution is inaccessible without NMR side chain assignments, MD simulations (covering time scales up to $\sim 150 \mathrm{~ns}$ ) were performed for both $w t$ mVDAC1 and mutated forms, which probe the importance of the charge and polarity of the side chain of E73: the

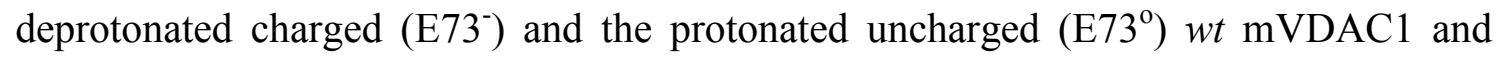
further the polar (E73Q) and hydrophobic (E73V) mutant forms. B-factors were calculated from the root-mean-square fluctuations (RMSF) observed in the MD simulations. In addition, to extract motions slower or faster than $1 \mathrm{~ns}$, a low-pass or high-pass filter, respectively, was employed. The analysis showed that in the fast regime ( $<1 \mathrm{~ns})$ the mobility of mVDAC1 was only marginally influenced by the charge of E73. In contrast, fluctuations occurring on time scales above $1 \mathrm{~ns}$ significantly increased from the uncharged to the charged form of E73 (Figure 30A). The rise in mobility was especially pronounced in $\beta$-strands $\beta 2-\beta 7$.

\footnotetext{
${ }^{5}$ The sections 3.3.6.1 and 3.3.6.2 contain results from MD simulations that were performed by Rodolfo Briones, Ulrich Zachariae, and Bert L. de Groot. The data is part of our collaborative publication (Villinger et al. 2010) and contains identical text passages.
} 

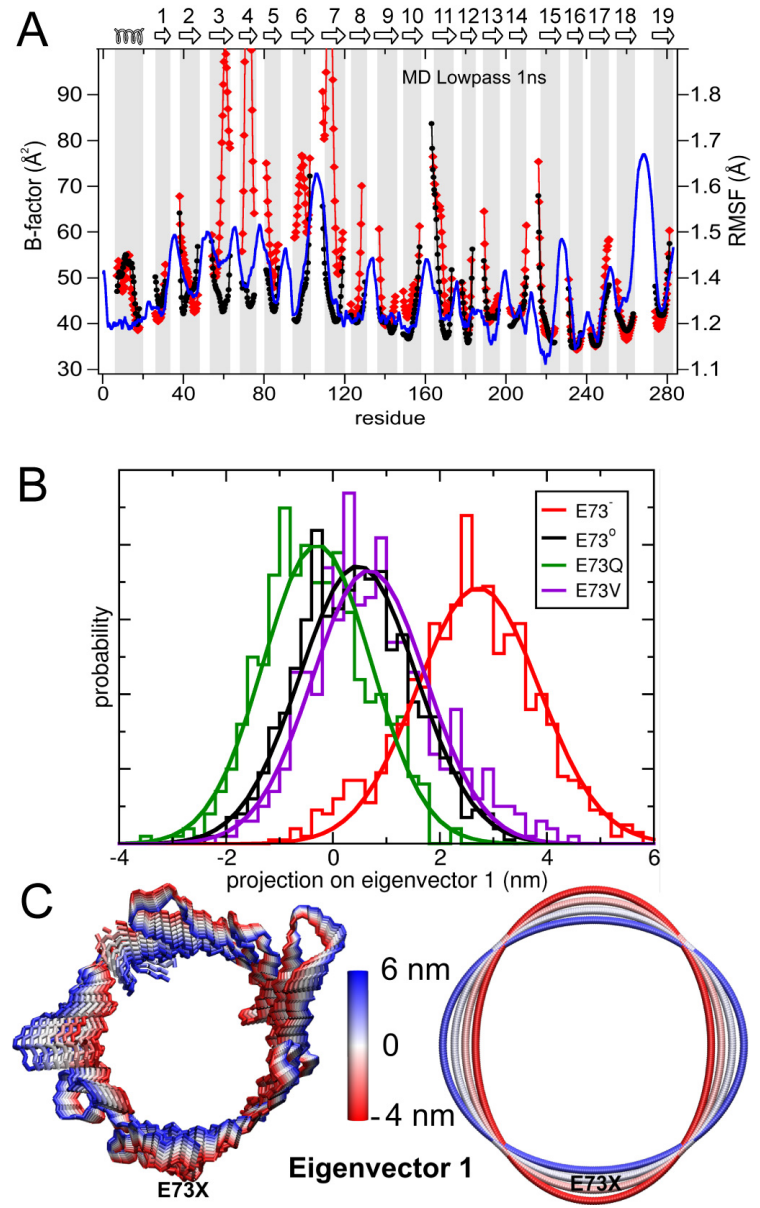

Figure 30. A charge on the membrane-facing E73 accounts for the elevation of $\mathrm{N}$-terminal protein dynamics of VDAC1. (A) Low pass filtered (1 ns) root-mean-square-fluctuations (RMSF) of the backbone of E73 (red) and E73 ${ }^{\circ}$ (black) mVDAC1 incorporated into a DMPC lipid bilayer observed during $150 \mathrm{~ns}$ MD simulations. Loop residues were not included. Crystallographic B-factors of mVDAC1 $(3 E M N)$ are shown in blue. The topology of mVDAC1 is indicated on top with numbered $\beta$-strands displayed as arrows. (B) PCA projection along the first eigenvector of mVDAC1, generated from the combined MD trajectories of all four E73 variants, using the backbone atoms of the $\beta$-barrel (residues 26283). Normalized histograms and Gaussian fits of the PCA projection show the extent of space of eigenvector 1 spanned by E73- (red), E73 ${ }^{\circ}$ (black), E73Q (green) and E73V (purple) trajectories. (C) Interpolation of the structure of mVDAC1 along the first PCA eigenmode using a backbone representation (left) and an elliptic fitting (right) of the $\mathrm{C} \alpha$ atoms of the backbone representation. The two extremes correspond to the two extremes in the histograms of E73- $(+6 \mathrm{~nm})$ and E73 $(-4 \mathrm{~nm})$ in (B). The figure was provided by Rodolfo Briones and reproduced from (Villinger et al. 2010).

To track global changes in the $\beta$-barrel, a principal component analysis (PCA) of the backbone dynamics was performed. A $1 \mathrm{D}$ projection of the $w t$ and mutant mVDAC1 trajectories onto the PCA eigenvector 1 (EV1) (Figure 30B) demonstrates that $\mathrm{E} 73^{\circ}$ and both neutral E73 mutants sampled a similar region of PCA space along the first eigenmode. In contrast, a charge on the side chain of E73 resulted in a larger coverage of space towards higher projections along Eigenvector 1 (Figure 30B). Eigenvector 1 describes the main differences between $w t$ and mutants, including 
differences in the average structure. A representation of the motion along the first eigenmode on the $\beta$-barrel and a fit of this mode to an ellipse (Figure 30C) showed that this mode can be viewed primarily as a global elliptic deformation of the barrel structure around E73.

\subsubsection{A negative charge on the side chain of E73 leads to thinning of the nearby} membrane

To further assess changes caused by mutations or protonation of E73, perturbations of the lipid bilayer in the MD simulations were analyzed. Comparison of the average bilayer thickness in the case of E73- and E73V (Figure 31A) demonstrates that a negative charge on the side chain of E73 leads to a marked thinning of the membrane around this residue. When the carboxylate group of E73 is uncharged by protonation $\left(E 73^{\circ}\right)$ no thinning occured, while the polar, but uncharged E73Q mutant displayed thinning to a lower extent.

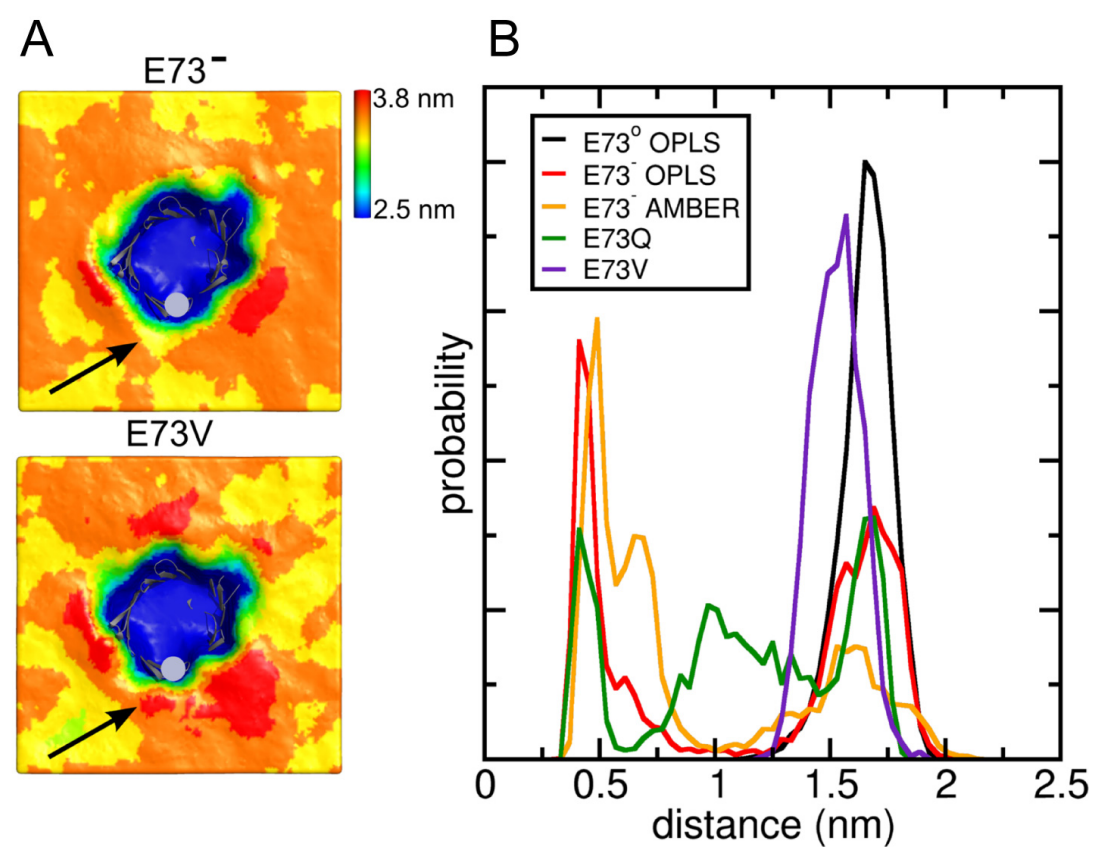

Figure 31. Membrane perturbation due to charge on E73. (A) Average thickness of DMPC bilayers calculated from MD simulations with mVDAC1 inserted. Upper panel, mVDAC1 E73- lower panel, mVDAC1 E73V mutant. The secondary structure of mVDAC1 is shown as grey cartoons and the position of E73 is indicated by a grey sphere. Arrows indicate the influenced membrane areas next to E73 and E73V. (B) Normalized minimum distance distribution between choline nitrogen atoms and E73. Probabilities are given in arbitrary units. The figure was provided by Rodolfo Briones and reprinted from (Villinger et al. 2010). 
This indicates that the commonly used replacement of glutamic acid with glutamine cannot fully mimic the uncharged form of E73 in VDAC1. Therefore the membrane deformation is attributed primarily to the presence of a charge at position 73 . The distortion of the membrane frequently correlated with a flipping motion of lipids in the bilayer, causing a transient approach of the phospholipid head groups towards E73. The flipping motion was reflected in an altered distribution of the distance between E73 and the closest lipid head group (Figure 31B). E73 ${ }^{-}$and E73Q substantially promoted the approach of lipid head groups from a distance of $\sim 1.5 \mathrm{~nm}$ to $\sim 0.5 \mathrm{~nm}$ to group 73 , i.e. into the central region of the membrane (Figure 31B). 


\section{4 hVDAC1 interactions}

The VDAC channel is the only known pathway for metabolites and small ions across the OMM (Benz 1994). VDAC transports ions with a preference for a range of large anions in its open state and an inverted selectivity for small cations like $\mathrm{Ca}^{2+}$ in the closed state (Blachly-Dyson and Forte 2001; Schein et al. 1976). With the 3D structure and NMR resonance assignments in solution available, interactions with transported substrates and ions can be investigated in detail. We have chosen to characterize the interaction sites of the major transport solute ATP and $\mathrm{Ca}^{2+}$ ions in order to gain insight into transport and gating of hVDAC1.

\subsubsection{Interaction of $\mathrm{hVDAC1}$ with nucleotides}

\subsubsection{ATP interacts with hVDAC1 at several sites}

Photoaffinity ATP labelling studies with rat VDAC1 (rVDAC1) in mitochondria or natively purified from mitochondria suggested the presence of three nucleotide binding sites (NBS): NBS1 in the $\alpha$-helix/linker region (residues 13-28), NBS2 in $\beta$-strand 7 (residues 110-120) and NBS3 in several C-terminal $\beta$-strands (residues 257-274/283) (Yehezkel et al. 2006). Further, magnesium was shown to reduce the binding affinity of ATP (Yehezkel et al. 2006). In order to determine ATP binding and $\mathrm{Mg}^{2+}$ inhibition with recombinantly expressed and refolded hVDAC1, we titrated hVDAC1 with ATP in form of the disodium salt $\left(\mathrm{Na}_{2} \mathrm{ATP}\right)$ and the magnesium complex (MgATP) and followed changes in ${ }^{1} \mathrm{H},{ }^{15} \mathrm{~N}$-TROSY spectra (Figure $32 \mathrm{~A}$ ). $\mathrm{Na}_{2} \mathrm{ATP}$ caused chemical shift differences (CSD) and resonance broadening even at low ratios of ATP, while MgATP had very little effect on hVDAC1 even at 64-fold excess (Figure 32). Upon $\mathrm{Na}_{2} \mathrm{ATP}$ addition, many residues exhibited ${ }^{1} \mathrm{H} /{ }^{15} \mathrm{~N}$ shifts and intensity changes at the same time (Figure 32).

Considering a $\mathrm{pK}_{\mathrm{a}}$ of 6.95 for $\mathrm{HATP}^{3-}$ (Smith and Alberty 1956a), at pH 6.8 and in the absence of $\mathrm{Mg}^{2+} \sim 41.5 \%$ of $\mathrm{ATP}_{\text {total }}$ is present in the deprotonated state $\left(\mathrm{ATP}^{4-}\right.$ ) and $58.5 \%$ as $\mathrm{HATP}^{3-}$, neglecting any bound $\mathrm{Na}^{+}$because of its weak affinity for ATP (Smith and Alberty 1956b). 

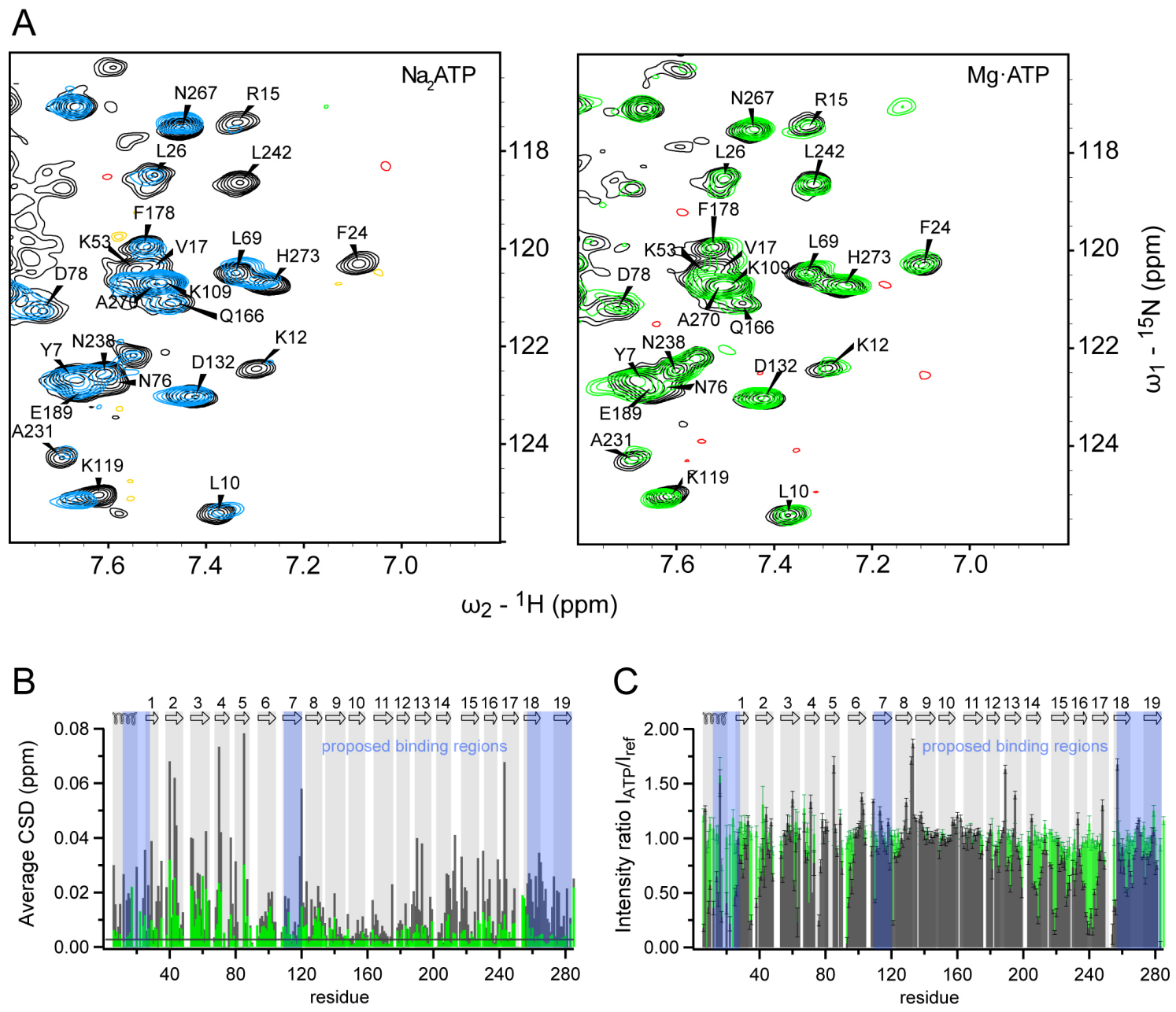

Figure 32. ATP titration of hVDAC1. Regions of ${ }^{1} \mathrm{H},{ }^{15} \mathrm{~N}-\mathrm{TROSY}$ spectra before (black) and after addition of $\mathrm{Na}_{2} \mathrm{ATP}$ at a ratio (hVDAC1:ATP) of 1:16 (light blue) or MgATP at a ratio of 1:64 (green). Average chemical shift deviations (CSD) (B) and scaled resonance intensity ratios $\mathrm{I}_{\mathrm{ATP}} / \mathrm{I}_{\mathrm{ref}}(\mathrm{C})$ obtained from ${ }^{1} \mathrm{H},{ }^{15} \mathrm{~N}$-TROSY spectra after addition of $\mathrm{Na}_{2} \mathrm{ATP}$ (grey) or MgATP (green) to hVDAC1 at a ratio of 1:16 (hVDAC1:ATP). The black line indicates the average CSD of non-shifting residues. Intensities were scaled relative to residues that were apparently unchanged by ATP addition (residues 142-145, 152-156, and 168-174). Previously proposed nucleotide binding regions (Yehezkel et al. 2006) are highlighted in blue, the topology of hVDAC1 is indicated on top and highlighted with grey bars.

In contrast, in the presence of a $10-20 \mathrm{mM}$ excess of $\mathrm{MgCl}_{2}$ a $\mathrm{MgATP}^{2-}$ population $>99 \%$ was calculated at all concentrations of $\mathrm{ATP}_{\text {total }}$ used in this study (Schoenmakers et al. 1992). Physiological $\mathrm{Mg}^{2+}$ concentrations are in the millimolar range (Akerboom et al. 1978; Traut 1994). Thus, hVDAC1 preferentially binds the free forms of ATP $\left(\mathrm{ATP}^{4-} / \mathrm{HATP}^{3-}\right)$. Small changes in CSD in the MgATP case result from a reduced affinity of the complexed form (fast exchange), traces of free ATP in equilibrium with the Mg-complexed form, or from sodium ions introduced by the stock solution. 
Since hVDAC1 displayed extensive dynamics in a range of time scales in the second part of the $\alpha$-helix, the linker and the N-terminal six $\beta$-strands (see section 3.3), one might expect that CSD and broadening are partly due to changes in the structure or dynamics of hVDAC1 rather than to pure chemical exchange from ATP interaction. However, due to the strong signal broadening and the absence or overlap of ligandbound resonances, experiments that rely on resonance intensities, (i.e. $\left\{{ }^{1} \mathrm{H}\right\}^{15} \mathrm{~N}$ HetNOEs and ${ }^{15} \mathrm{~N}$ transverse relaxation rates) will have low accuracy at these ATP concentrations. Experiments that rely on chemical shifts and report on the secondary structure rather than chemical environment (e.g. C $\alpha$ shifts) might be promising for determination of structural changes. However, the strong broadening of the amide resonances reduces the magnetization transfer efficiency to $\mathrm{C} \alpha$ resonances via an HNCA. Thus, an optimum ATP concentration is required that allows sufficient magnetization transfer and detectable chemical shift changes at the same time. Such optimization of experiments is currently ongoing. Alternative 2D carbon spectra are probably insufficient in terms of sensitivity and exhibit strong overlap that could only be resolved by a variety of selectively labelled samples. Hence, currently only amide CSD and resonance intensity changes are available for characterization of nucleotide interactions. For simplicity, all ATP-affected regions are termed ATP binding sites in the following and this issue is discussed later (see Discussion).

Residues that showed CSD or broadening upon addition of $\mathrm{Na}_{2} \mathrm{ATP}$ were distributed over a large area of the protein (Figure 32B, C). However, clusters of affected residues were observed in several regions. The strongest broadening together with many shifts was observed in the C-terminal $\beta$-strands $\beta 12-\beta 19$ as well as residues in the $\alpha$-helix and the linker (Figure 32, Figure 33A, and Table 7). Residues in the Nterminal $\alpha$-helix and linker are in close proximity to strands $\beta 12-\beta 19$ in the $3 \mathrm{D}$ structure of hVDAC1 and can be considered as one ATP binding region (Figure 33A, B). This binding site contains the proposed NBS1 and 3, although the strongest effect in the Cterminal strands $(\beta 15-\beta 17)$ in solution is slightly shifted compared to the NBS3 (Figure 32B, C). This offset can be explained by the large size of the ATP binding site and a preferred orientation and cross-linking region $(\beta 18-\beta 19)$ of the photoreactive ATP analogue upon binding to VDAC1. 

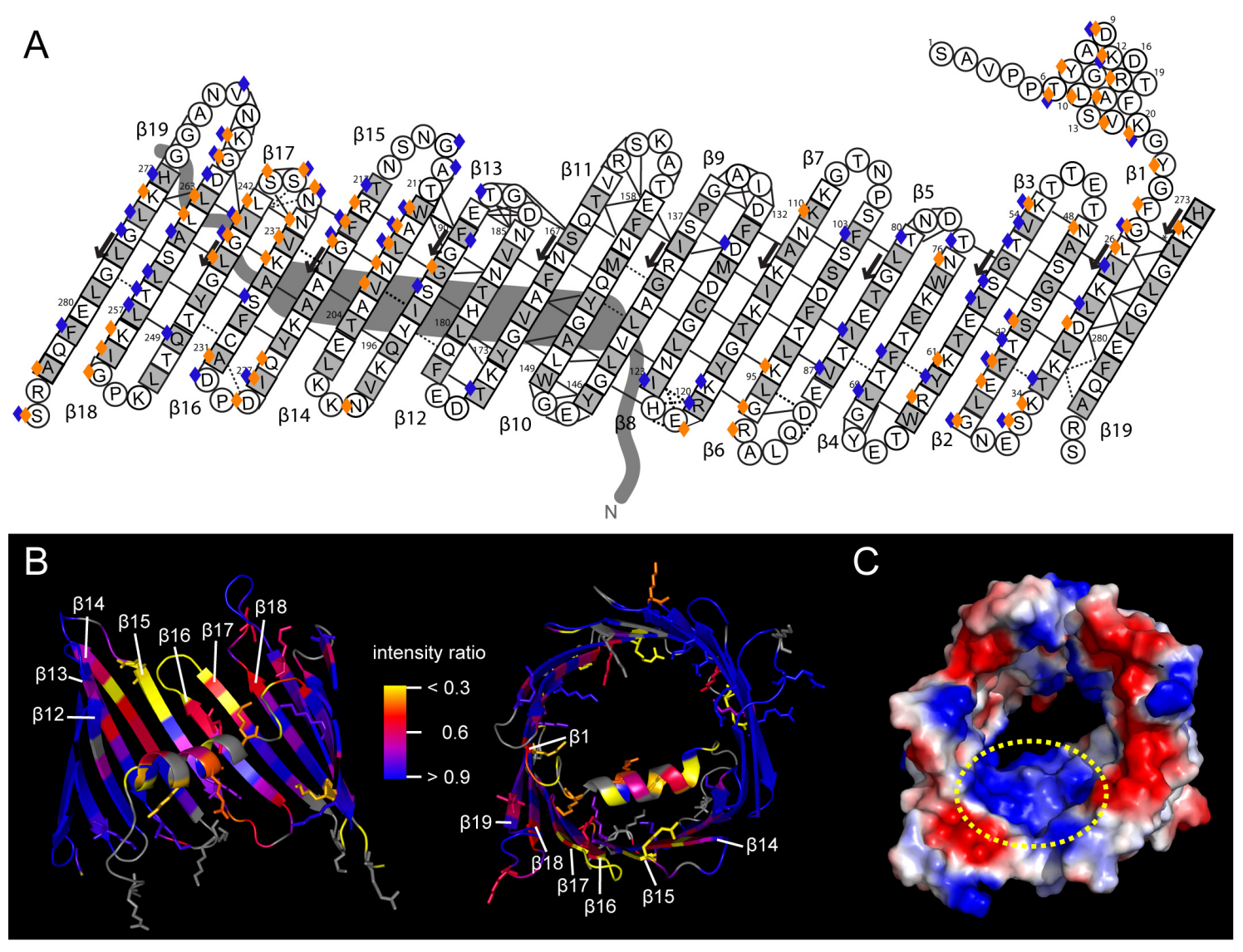

Figure 33. C-terminal ATP binding site on VDAC1. (A) Topology map of hVDAC1 (adapted from (Bayrhuber et al. 2008)) showing residues affected by a 16-fold excess of $\mathrm{Na}_{2} \mathrm{ATP}$. Boxed residues form $\beta$-strands with outward pointing residues are coloured in grey. Encircled residues are located in loops or the N-terminal $\alpha$-helix. Residues displaying average chemical shift deviations larger than 0.02 (orange diamonds) or broadened below intensity ratio values of 0.7 (blue diamonds) upon 16-fold excess of $\mathrm{Na}_{2} \mathrm{ATP}$ are indicated. Grey tubes in the back indicate the approximate location of the N-terminal $\alpha$ helix. (B) Resonance broadening induced by 16-fold excess of ATP mapped onto the structure of hVDAC1 (PDB code: 2JK4), inside and "top" view. Resonance broadening is colour-coded, with effects increasing from blue (not affected) to yellow (strongly broadened). Non-assigned or non-resolved residues are coloured in grey. Lysine and arginine side chains are presented as sticks. (C) Electrostatic potential map of mVDAC1 (PDB code: 3EMN) calculated with Delphi (Accelrys), showing regions with positive (blue) and negative (red) potential. The patch of residues broadened upon ATP addition is encircled in yellow.

In conclusion, the C-terminal ATP binding region agrees with the proposed NBS1 and includes NBS3 (Yehezkel et al. 2006). The C-terminal ATP binding site is very distinctive and specific (Figure 33A). The same region also exhibits a positive electrostatic potential (Figure 33C) that might be responsible for interaction with the negatively charged free $\mathrm{ATP}^{4-} / \mathrm{HATP}^{3-}$.

Shifts and broadening in L5-6/ß6 (residues 90-96), as well as in the adjacent strands $\beta 7-\beta 8$ (residues 116-127), indicate the presence of a second ATP binding site (Figure 34) close to the proposed NBS2 ( $\beta 7$ ) (Yehezkel et al. 2006). 
Table 7. Residues strongly broadened by ATP.

\begin{tabular}{ll}
\hline Secondary structure element & Residues affected by ATP \\
\hline N-terminal helix & T6, A8-L10, K12, A14-R15, V17 \\
Linker & K20, Y22, F24-L26 \\
$\beta 12$ & H181, N185 \\
$\beta 13$ & F190, G192-S193, V198-N199 \\
$\beta 14$ & E203, V206-T211 \\
$\beta 15$ & N216-G220, A222, K224-D228 \\
$\beta 16$ & D230-C232, A235-N238 \\
L16-17 & N239-S241 \\
$\beta 17$ & L242-L245, Q249 \\
$\beta 18$ & G254-K256, L259, A261-L263 \\
L18-19 & G265-K266 \\
$\beta 19$ & K274, G276, E280-F281, A283 \\
C-terminus & S285 \\
\hline
\end{tabular}

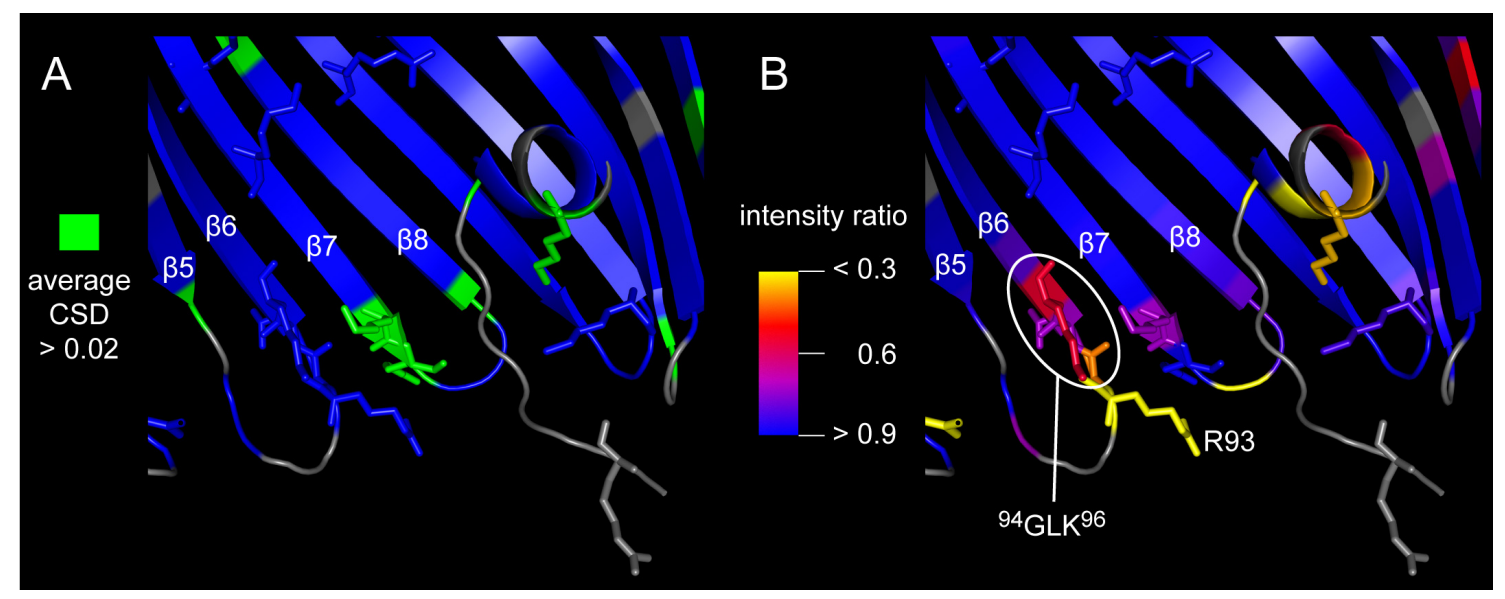

Figure 34. ATP interaction with the GLK motif. Average CSD (A) and broadening (B) induced by 16fold excess of ATP are mapped onto the hVDAC1 structure (PDB code: 2JK4) shown from the inside. Lysine and arginine residues are shown as sticks. Residues with an average CSD larger than 0.02 are mapped onto the structure in green. Resonance broadening is colour-coded, with effects increasing from blue (not affected) to yellow (strongly broadened). Non-resolved residues are coloured in grey.

This region contains a conserved glycine-leucine-lysine (GLK) motif (residues 94-96) proposed to be important for ion selectivity, but dispensable for ATP binding (Runke et al. 2000) (Figure 34B). However, only few residues are affected, the strongest being R93 in the loop (Figure 34B), suggesting a minor role for this ATP binding site.

In addition, the $\mathrm{N}$-terminal strands $\beta 1-\beta 5$ were affected by $\mathrm{Na}_{2} \mathrm{ATP}$ and to a minor extent also by MgATP, inducing chemical shift changes or broadening in several residues scattered over a broad region (Figure 32 and Figure 35). Together with the GLK motif these residues might form a second (N-terminal) ATP binding site. 


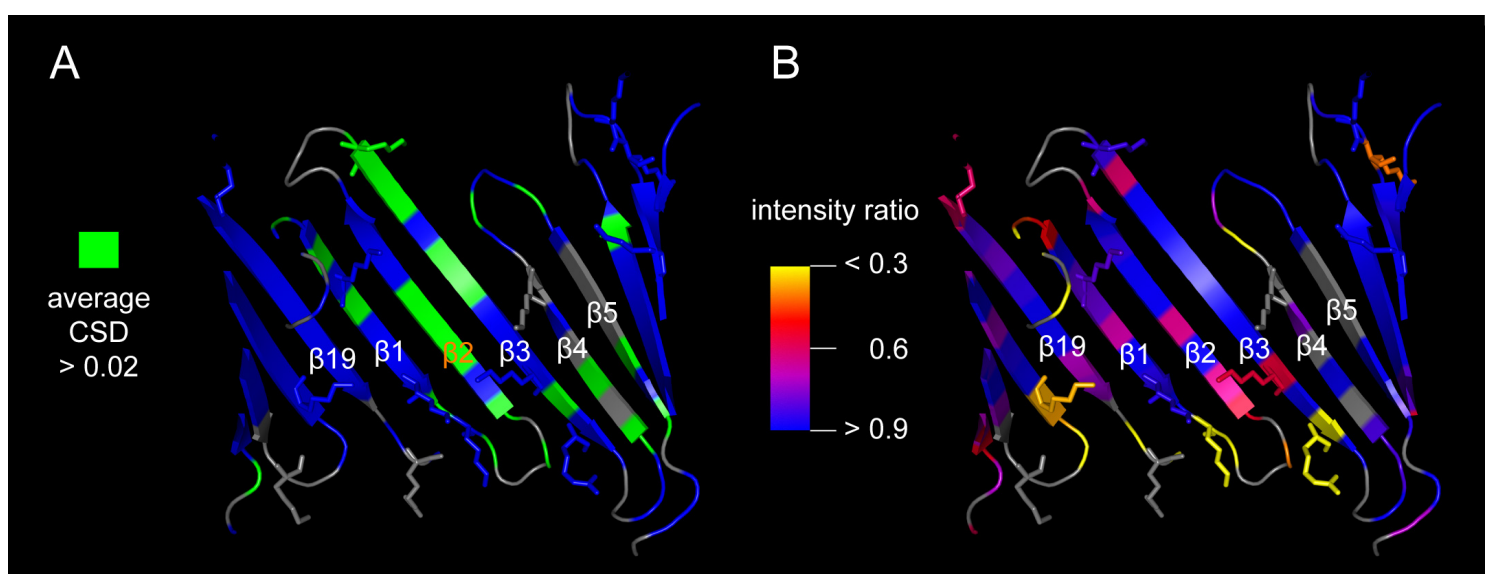

Figure 35. $\mathbf{N}$-terminal $\boldsymbol{\beta}$-strands of hVDAC1 are affected by ATP. Average CSD (A) and broadening (B) induced by 16-fold excess of ATP are mapped onto the hVDAC1 structure (PDB code: 2JK4), displayed from the inside. Lysine and arginine residues are shown as sticks. Residues with an average CSD larger than 0.02 are mapped onto the structure in green. Resonance broadening is colour-coded, with effects increasing from blue (not affected) to yellow (strongly broadened). Non-resolved residues are coloured in grey.

\subsubsection{Affinity of the ATP interaction}

For residues in the fast exchange regime on the NMR time scale (i.e. the rate of binding exchange is faster than the difference in chemical shifts of the bound and free state), dissociation constants $\left(\mathrm{K}_{\mathrm{D}}\right)$ can be estimated. This is achieved by fitting a two-state binding model to the variation of average CSD values with increasing ligand concentration (Craik and Wilce 1997). Most residues in the two ATP binding sites of hVDAC1 exhibited shifts and broadening at the same time (Figure 32B, C), indicating that ATP binding occurs on the fast-to-intermediate NMR time scale (i.e. the exchange rate of binding is higher or of the same order as the chemical shift differences). In addition, some resonances might be in slow exchange, although resonances of the bound state were missing or overlapped. Intermediate exchange contributions to chemical shifts introduce large errors in $K_{D}$ value determination from fast exchanging residues, and can lead to an underestimation of the true value by two orders of magnitude (Feeney et al. 1979). Therefore, only residues were included in the analysis that were most likely in the fast exchange regime (i.e. residues that exhibited signal broadening less than $20 \%$ in the final titration step after intensity scaling). Nevertheless, the present data allows only crude estimation of the dissociation constant for ATP, since the contribution from intermediate exchange cannot be fully excluded. Examples of shifting residues and predicted $\mathrm{K}_{\mathrm{D}}$ values for the $\mathrm{N}$ - and $\mathrm{C}$-terminal ATP binding site 
are shown in Figure 36. The plots of average CSD versus ATP concentration demonstrate that saturation was nearly reached. A similar analysis of dissociation constants was performed for 21 residues in the C-terminal ATP binding site, averaging to a $K_{D}$ of $(1.15 \pm 0.11) \mathrm{mM}$ for the $\mathrm{Na}_{2} \mathrm{ATP}$ interaction. The affinity for $\mathrm{Na}_{2} \mathrm{ATP}$ in the $\mathrm{N}$-terminal binding site (including the GLK motif) was estimated from ten residues, resulting in an average $K_{D}$ of $(0.80 \pm 0.12) \mathrm{mM}$. However, all residues used for the latter analysis either exhibited overlap or large deviations in one or two data points. Together with the scattered effects in the N-terminal binding region and the potential error from intermediate exchange contributions (Feeney et al. 1979), the affinity for the $\mathrm{N}$-terminal binding site is less reliable than for the C-terminal binding region. Moreover, the extensive broadening in the C-terminal binding region (Figure 33) suggests that this region has a higher affinity for ATP, assuming that ATP induced CSD are of similar magnitude in both binding regions.

A
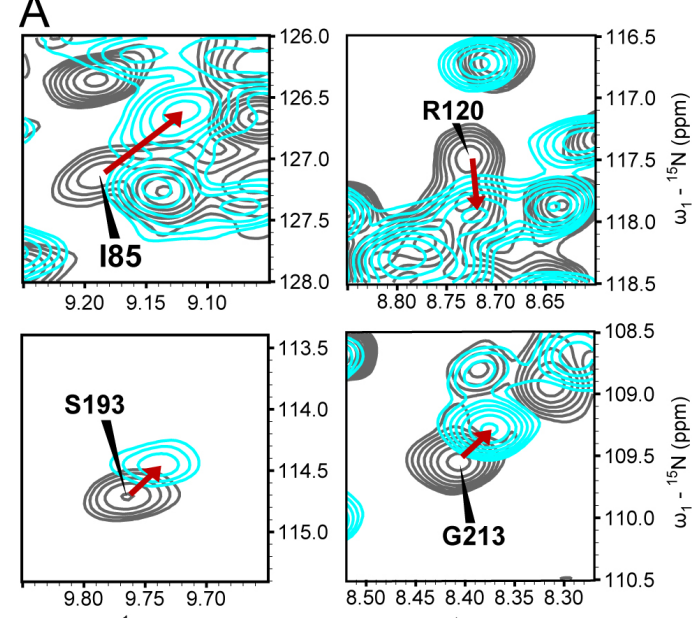

$\omega_{2}-{ }^{1} \mathrm{H}(\mathrm{ppm})$

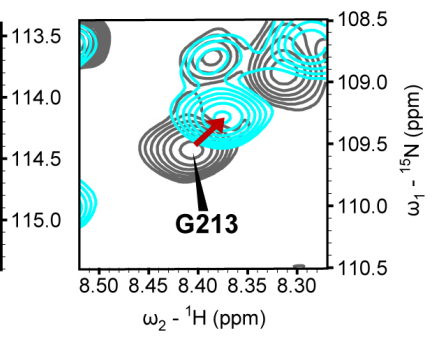

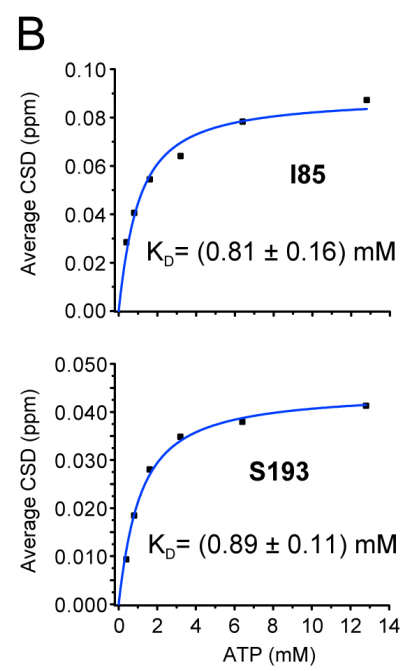

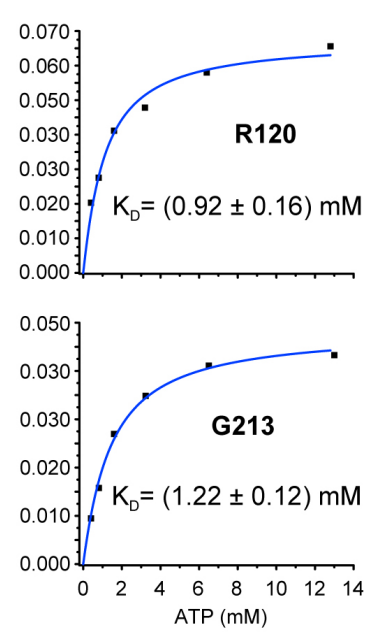

Figure 36. Affinity of hVDAC1 for ATP. (A) Spectral regions of ${ }^{1} \mathrm{H},{ }^{15} \mathrm{~N}-\mathrm{TROSY}$ spectra before (grey) and after (cyan) addition of a 32 -fold excess of $\mathrm{Na}_{2}$ ATP showing examples of residues shifting strongly in the presence of ATP. (B) Plot of averaged chemical shift deviation (CSD) values versus ATP concentration and curve fits for $\mathrm{K}_{\mathrm{D}}$ determination using a one-site binding model. Resonance intensity ratios, scaled with respect to apparently non-exchanging residues (residues 142-145, 152-156, and 168174), were 2.3 (I85, overlapped with a second resonance), 0.98 (R120), 0.82 (S193), and 1.0 (G213) for the last titration step, showing that these residues were most likely in the fast exchange regime on the NMR time scale.

\subsubsection{Influence of the ionic concentration}

To probe for effects from an elevated ionic concentration introduced by counter ions in the nucleotide stock solution, we tested the influence of $\mathrm{NaCl}$ on ${ }^{1} \mathrm{H},{ }^{15} \mathrm{~N}$-TROSY 
spectra of hVDAC1. For this purpose, 50 and $100 \mathrm{mM}$ concentrations were used, which are of the same order or higher, respectively, than the estimated salt concentration in the $\mathrm{Na}_{2}$ ATP titration (e.g. at 32-fold ATP excess (12.8 mM ATP), about $40 \mathrm{mM} \mathrm{Na}$ counter ions were present). $50 \mathrm{mM} \mathrm{NaCl}$ introduced shifts in $\beta 3-\beta 6$ (Figure 37A) and changes in intensities in a few residues in $\beta 1-\beta 5$ and the very $\mathrm{C}$-terminus (Figure $37 \mathrm{~B}$ ). Thus, the observed ATP induced shifts and broadenings in the N-terminal region (Figure 32) are partly caused by the elevated ionic concentration, while the effects in the C-terminal ATP binding site ( $\mathrm{N}$-terminal $\alpha$-helix, linker, C-terminal $\beta$-strands $\beta 12-\beta 19)$ are purely related to the presence of the nucleotide.

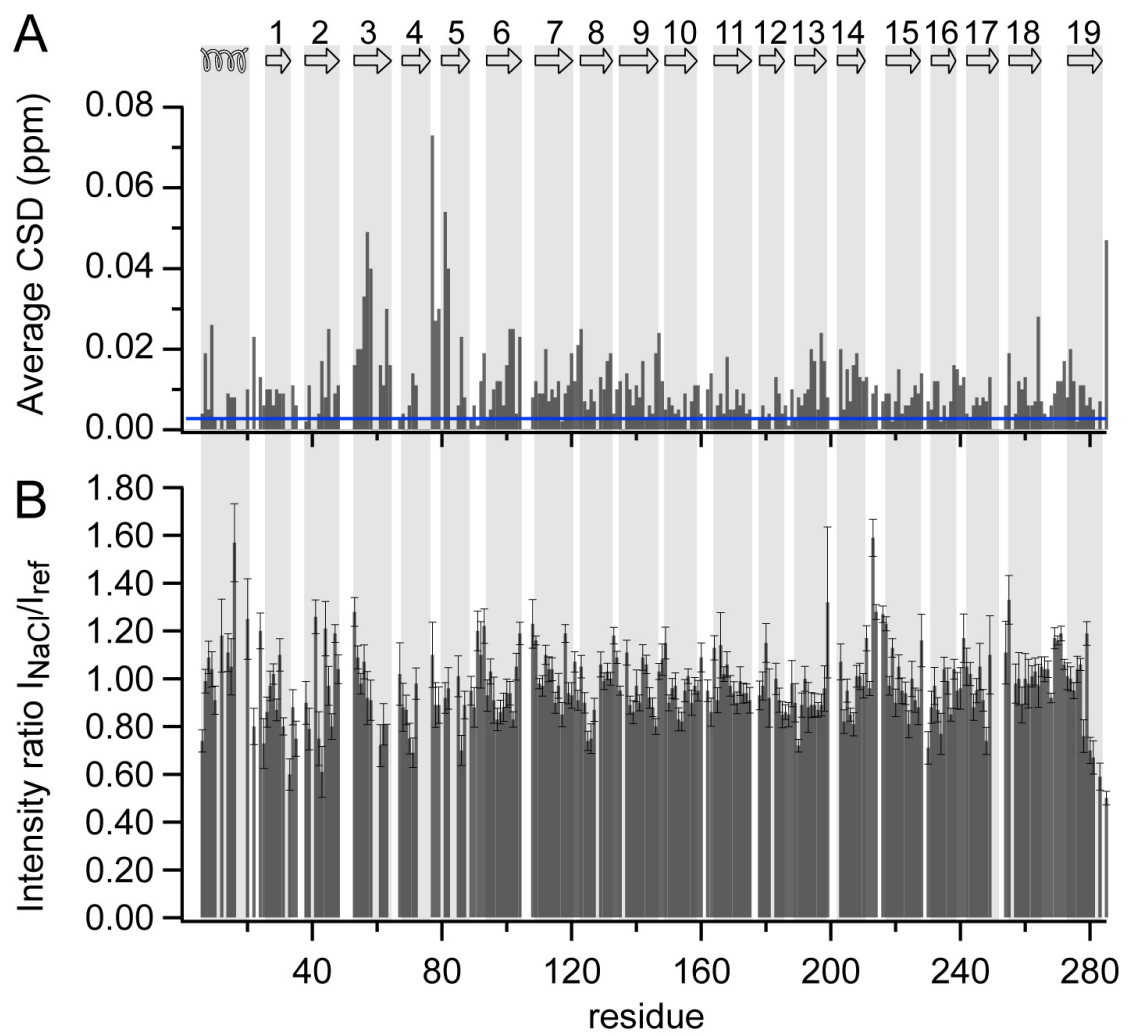

Figure 37. Ionic concentration affects residues in the N-terminal barrel region of hVDAC1. Average chemical shift deviations (CSD) (A) and resonance intensity ratios $\mathrm{I}_{\mathrm{NaCl}} / \mathrm{I}_{\mathrm{ref}}$ (value \pm standard error) (B) obtained from ${ }^{1} \mathrm{H},{ }^{15} \mathrm{~N}$-TROSY spectra after addition of $50 \mathrm{mM} \mathrm{NaCl}$ to a mutant $(\mathrm{T} 60 \mathrm{C} / \mathrm{C} 127 \mathrm{~S} / \mathrm{C} 232 \mathrm{~S})$ $\mathrm{hVDAC1}$. The blue line in (A) indicates the average CSD of non-shifting residues.

\subsubsection{4 hVDAC1 binds other nucleotides as well}

VDAC1 is able to bind other nucleotides as well (Yehezkel et al. 2006). Therefore we tested the effect of two other nucleotides (GTP and UTP) on hVDAC1. Both UTP and GTP induced CSD and resonance broadening in similar regions as ATP: the linker, 
some $\alpha$-helix residues, and $\beta 12-\beta 19$ (C-terminal binding site) as well as $\beta 1-\beta 8(\mathrm{~N}-$ terminal binding site) (Figure 38). Thus, the same regions in hVDAC1 are involved in binding of various nucleotides.

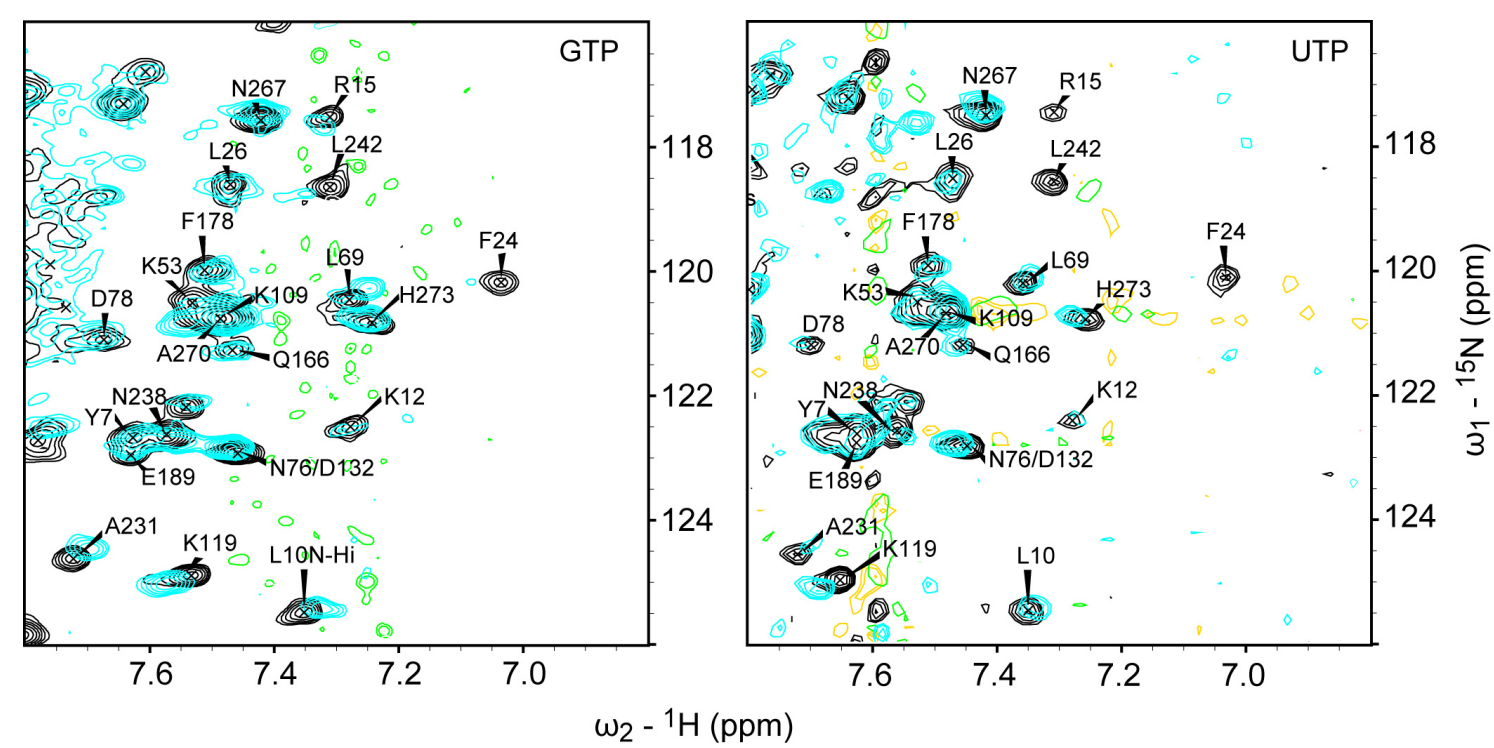

Figure 38. UTP and GTP binding of hVDAC1. Regions of ${ }^{1} \mathrm{H},{ }^{15} \mathrm{~N}-\mathrm{TROSY}$ spectra identical to the region shown in Figure 32, before (black) and after (cyan) addition of GTP or UTP at hVDAC1:nucleotide ratios of 1:16 (GTP) or 1:32 (UTP).

\subsection{2 $\mathrm{Ca}^{2+}$ interaction with hVDAC1}

\subsubsection{Binding of $\mathrm{Ca}^{2+}$ to $\mathrm{hVDACl}$}

Two glutamic acid residues, E73 and E203, have been proposed as $\mathrm{Ca}^{2+}$-binding sites for VDAC1 on the basis of mutation studies and photoreactive cross-linking of ruthenium compounds (Israelson et al. 2007). Furthermore, $\mathrm{Ca}^{2+}$ has been proposed to induce channel opening (Bathori et al. 2006), a process that requires specific $\mathrm{Ca}^{2+}$ binding sites. In order to determine the location of these $\mathrm{Ca}^{2+}$-binding sites in hVDAC1 we added increasing concentrations of $\mathrm{CaCl}_{2}$ to micellar solutions of $0.6 \mathrm{mM}{ }^{2} \mathrm{H} /{ }^{15} \mathrm{~N}$ labeled hVDAC1 and followed changes of the amide backbone resonances in ${ }^{1} \mathrm{H},{ }^{15} \mathrm{~N}$ TROSY spectra. Previously, no interaction of hVDAC1 with $\mathrm{Ca}^{2+}$ was detected (Bayrhuber 2007). Re-evaluation of the data with the improved assignment, however, enabled the detection of a weak effect. At 16-fold excess of $\mathrm{Ca}^{2+}(9.6 \mathrm{mM})$ small changes in peak intensities and chemical shifts were observed for the linker connecting the $\alpha$-helix to the $\beta$-barrel and the N-terminal six $\beta$-strands (Figure 39A, B). 

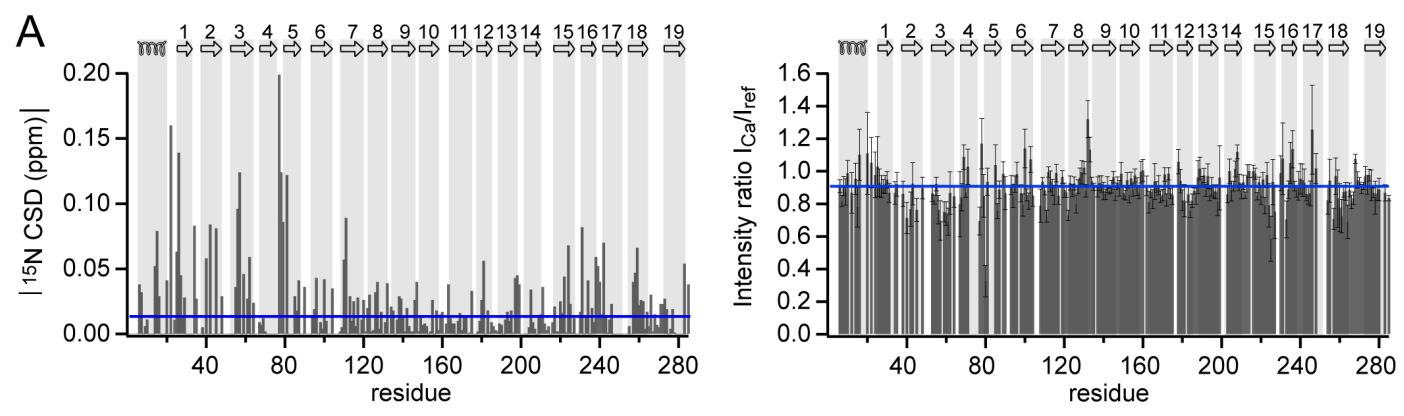

B
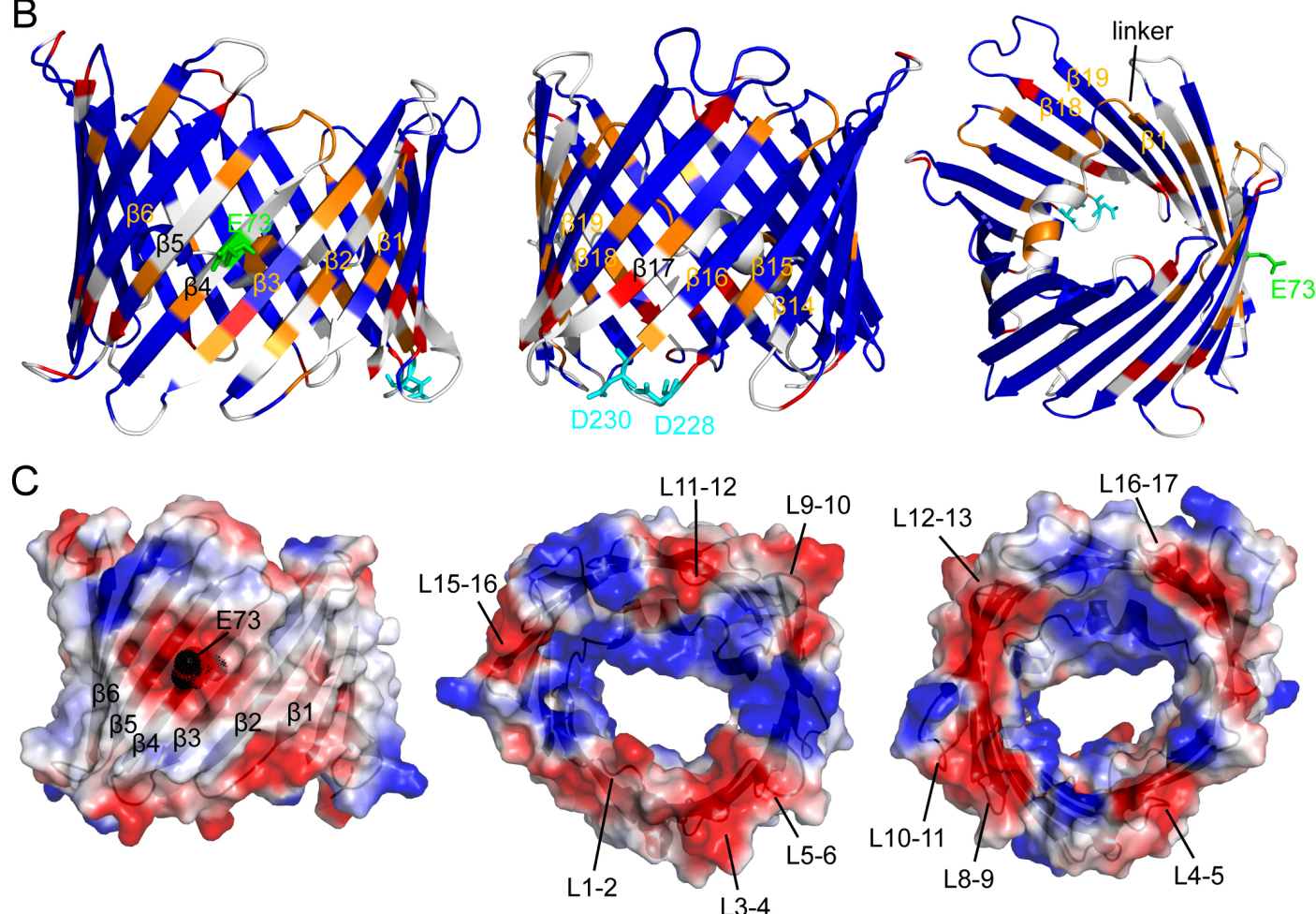

Figure 39. Calcium affected regions in hVDAC1. (A) Changes in magnitude of ${ }^{15} \mathrm{~N}$ chemical shift deviation (CSD) and peak intensity (value \pm standard error) observed in ${ }^{1} \mathrm{H},{ }^{15} \mathrm{~N}-\mathrm{TROSY}$ spectra of hVDAC1 induced by a 16-fold excess of $\mathrm{CaCl}_{2}$ (spectra were provided by Monika Bayrhuber). The blue line in the left graph indicates the average magnitude of ${ }^{15} \mathrm{~N}$ CSD threshold for non-shifting residues. The blue line in the right graph shows the average value of intensity ratios (0.91) for residues 90-200. The topology of hVDAC1 is shown on top with secondary structure elements highlighted in grey. (B) Residues with a magnitude of ${ }^{15} \mathrm{~N}$ CSD larger than $0.05 \mathrm{ppm}$ (orange) and broadening below a ratio of 0.8 (red) at a 1:16 ratio of hVDAC1:Ca ${ }^{2+}$ mapped onto the structure of mVDAC1 (PDB code: 3EMN). Residues not observable or not assigned in $w t$ hVDAC1 are coloured white. (C) The electrostatic potential map calculated with DelPhi (Accelrys) is mapped onto the surface of the structure of mVDAC1 in different orientations (side view as in B, top-down and down-top view). A black sphere indicates E73.

The overall reduction of peak intensities $(9 \%$ for the central $\beta$-strands, Figure 39A) is likely due to a sensitivity loss of the cryogenic probe at the elevated ionic concentration. At $9.6 \mathrm{mM} \mathrm{Ca}^{2+}$ concentrations the most affected signals (in terms of position and intensity) were Y22 and L26 (linker), K34 ( $\beta 1)$, E40, T42, and G45 ( $\beta 2$ ), G56, S57, E59, and K61 ( $\beta 3)$, F71 ( $\beta 4)$, T77, D78, N79, and T80 (loop 4-5), L81 ( $\beta 5)$, 
N111 ( $\beta 7$ ), and H122 (loop L7-8). In addition, smaller chemical shifts and intensity changes occurred for residues in the N-terminal $\alpha$-helix (A14, R15) and the C-terminal strands $\beta 15-\beta 18$ (residues affected at 16-fold $\mathrm{Ca}^{2+}$ excess: K224, Y225, and I227 ( $\left.\beta 15\right)$, A231, F233, and N238 ( $\beta 16)$, N239 (loop 16-17), L242 and G246 ( $\beta 17)$, L257, L259, A261, and D264 ( $\beta 18)$ ). In order to approach saturation for the C-terminal binding site, the calcium concentration was increased to a 64-fold excess. However, at this excess of $\mathrm{CaCl}_{2}$ small changes in NMR signal position were distributed over the entire protein (data not shown), indicating non-specific effects on charged residues induced by the elevated ionic concentration. The regions most affected by $\mathrm{Ca}^{2+}$ coincide with patches of negative potential (e.g. $\beta 3-5$, L4-5 and L15-16 containing D228 and D230, and L1617) (Figure 39C). Since $\mathrm{Ca}^{2+}$ did not affect other regions - as far as assigned - with a negative potential (e.g. L8-9, L10-11, L12-13), the effects induced in hVDAC1 are specific.

As shown before, the $\mathrm{N}$-terminal strands $\beta 3-\beta 5$ are affected by $\mathrm{NaCl}$ (Figure 37). However, the $\mathrm{Ca}^{2+}$ affected region in the C-terminus ( $\left.\beta 15-\beta 18\right)$, the linker, and $\beta 1$ $\beta 2$ were unaffected by $\mathrm{NaCl}$ (Figure 37). Although $\mathrm{Ca}^{2+}$ interaction involves L16-17 in the centre of the C-terminal ATP binding site (Figure 33), slightly more effects were observed on the opposite barrel end near L15-16 (Figure 39B). Furthermore, the $\mathrm{Ca}^{2+}$ effects in the C-terminal strands $\beta 15-\beta 18$ (Figure 39A) are much weaker than the ATP induced effects in the C-terminal ATP binding site (Figure 33). As stressed for the interaction with ATP, CSD and intensity changes might result from structural or dynamic changes. However, since the effects in this case are even smaller and located in regions of low signal intensities, changes in ps-ns or $\mu s-m s$ dynamics, and $\mathrm{C} \alpha$ shifts are more difficult to explore by solution NMR.

A possibility for the small spectral changes could be the presence of $\mathrm{Ca}^{2+}$ traces tightly bound to hVDAC1. To probe for the presence of $\mathrm{Ca}^{2+}$ traces in hVDAC1 preparations we added EGTA, followed by an excess of $\mathrm{CaCl}_{2}$. Addition of EGTA induced strong chemical shifts and resonance broadening (Figure 40). The affected residues were located in the $\alpha$-helix, the linker, $\beta 13-\beta 18$ (with the strongest effects in L16-17), as well as $\beta 1-\beta 2$ (Figure 40), and hence involve the same residues that form the C-terminal ATP binding site in hVDAC1 (Figure 33). 


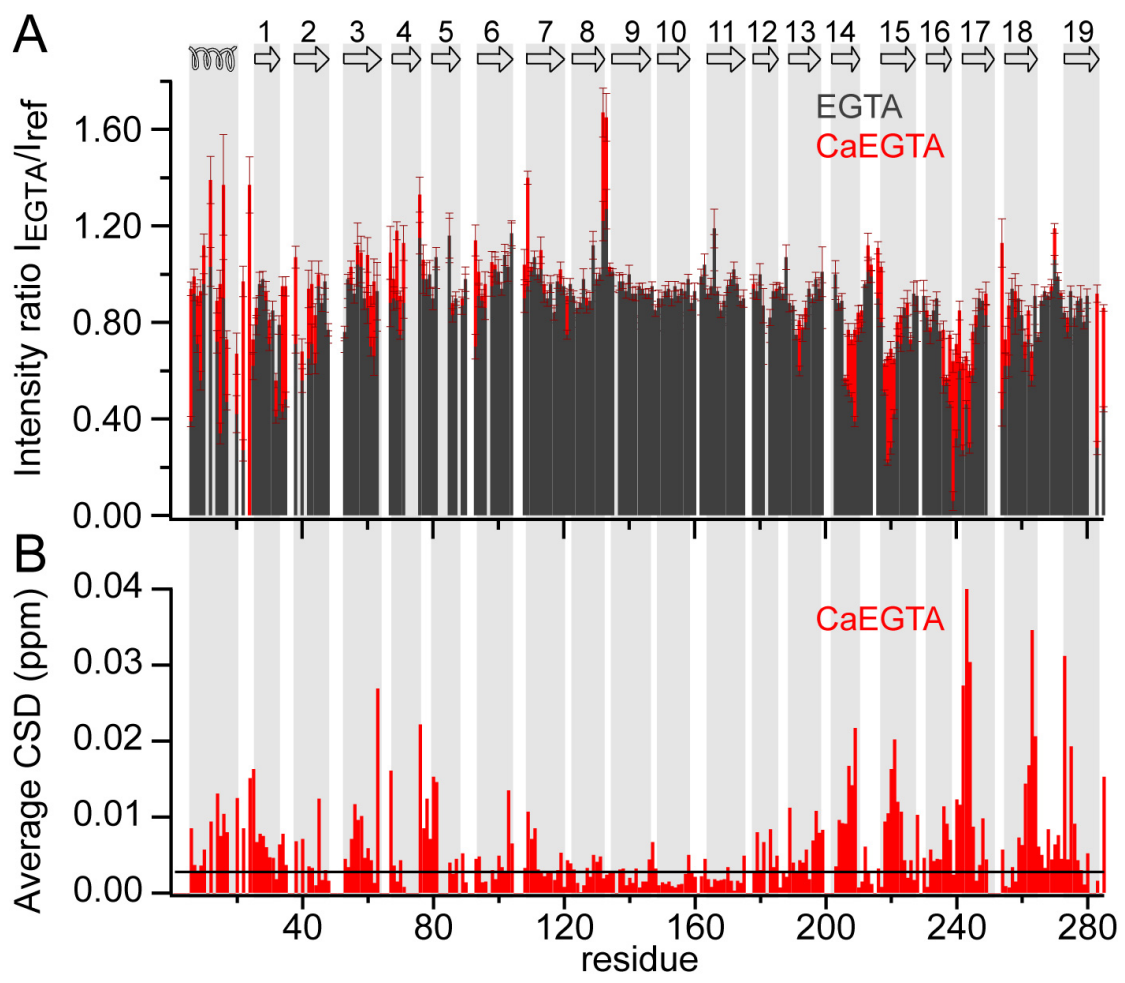

Figure 40. EGTA interaction with hVDAC1. (A) Resonance intensity ratios obtained from ${ }^{1} \mathrm{H},{ }^{15} \mathrm{~N}$ TROSY spectra after addition of $5 \mathrm{mM}$ EGTA to hVDAC1 (grey) followed by an excess of $\mathrm{CaCl}_{2}$ (free $\mathrm{Ca}^{2+}:$ hVDAC1 $4: 1$ ) (red). (B) Average CSD obtained after $\mathrm{CaCl}_{2}$ addition compared to the EGTA containing sample. The black line indicates the average CSD of non-shifting residues. The topology of $\mathrm{hVDAC1}$ is indicated on top and highlighted with grey bars.

This suggests a direct interaction of EGTA with hVDAC1 in the C-terminal ATP binding region. Addition of $\mathrm{CaCl}_{2}$ reduced the broadening and induced previously absent chemical shift deviations in the same residues, with the strongest effects again in L16-17 (Figure 40). This indicates a switch between strong binding of free EGTA (slow-intermediate exchange) to lower affinity binding of the CaEGTA complex (intermediate-fast exchange), similarly to the free and complexed form of ATP (Figure 32). Hence, $\mathrm{Ca}^{2+}$ effects cannot be distinguished from the potential binding of EGTA to the previously identified C-terminal ATP binding site. As an alternative to obtain a $\mathrm{Ca}^{2+}$-free sample, we intended to remove free $\mathrm{Ca}^{2+}$ and CaEGTA by dialysis. Although the dialysis was not completed even after 16 hours at room temperature, most of the broadening effects were alleviated and the intensity profiles were similar to the CaEGTA condition (data not shown).

Taken together we conclude that $\mathrm{Ca}^{2+}$ binds to the $\mathrm{N}$-terminal five $\beta$-strands of hVDAC1, and a second interaction site might be located within $\beta 15-18$. The $\mathrm{C}$-terminal binding site involves residues of the linker, as is the case for ATP. The first binding site 
contains E73 in $\beta 4$ and thus agrees with previous observations (Israelson et al. 2007). Although a second residue suggested to participate in $\mathrm{Ca}^{2+}$-binding, E203, is located on the same barrel end as the majority of effects in $\beta 15-\beta 18$, its location is only next to the residues that exhibit the strongest effects. Therefore, E203 is most likely not directly involved in the interaction.

\subsubsection{Lanthanide ions confirm the C-terminal $\mathrm{Ca}^{2+}$-interacting region}

Due to their similarity in ionic radii, coordination geometry and donor atom preference (Martin and Richardson 1979), lanthanide ions can be used to determine calcium binding sites in proteins. Lanthanides have been shown to interact with various $\mathrm{Ca}^{2+}$ binding proteins (Hadad et al. 1994; Reed and Bygrave 1974) and VDAC1, for which they induce channel closure and inhibit $\mathrm{Ca}^{2+}$ transport (Gincel et al. 2001). An advantage of paramagnetic lanthanide ions like $\mathrm{Gd}^{3+}$ is that they induce paramagnetic relaxation enhancement (PRE), such that direct binding can be established from resonance broadening and can be distinguished from structural changes in the protein. Since CSD and intensity changes cannot distinguish binding from structural changes, $\mathrm{Gd}^{3+}$ provides a useful alternative. $\mathrm{GdCl}_{3}$ was used previously to obtain further information about the $\mathrm{Ca}^{2+}$-binding sites in hVDAC1 (Bayrhuber 2007) and we now increased the accuracy with the help of the improved assignment. Addition of $\mathrm{GdCl}_{3}$ at a hVDAC1: $\mathrm{Gd}^{3+}$ ratio of 1:0.25 led to an overall reduction of peak intensities by $33 \%$ due to the paramagnetic solute (Figure 41A). On top of the overall broadening, a distinct subset of peaks was broadened more than 50\% (E203, C232, Y247, Q249, L257) or beyond detection (N199, K224, Y225, Q226, I227, F233, T248, L259) (Figure $41 \mathrm{~A}, \mathrm{~B})$. Thus, as determined previously (Bayrhuber 2007), the location of the $\mathrm{Gd}^{3+}$ binding site in $\beta$-strands $\beta 15-\beta 18$ was confirmed with the updated assignment of hVDAC1. These data agree well with the C-terminal regions at the lower barrel end affected by $\mathrm{CaCl}_{2}$ (Figure 39). Thus the data estabilish the $\mathrm{C}$-terminal $\mathrm{Ca}^{2+}$-binding site located on the barrel end opposite to the C-terminal ATP binding site (Figure 41C). 

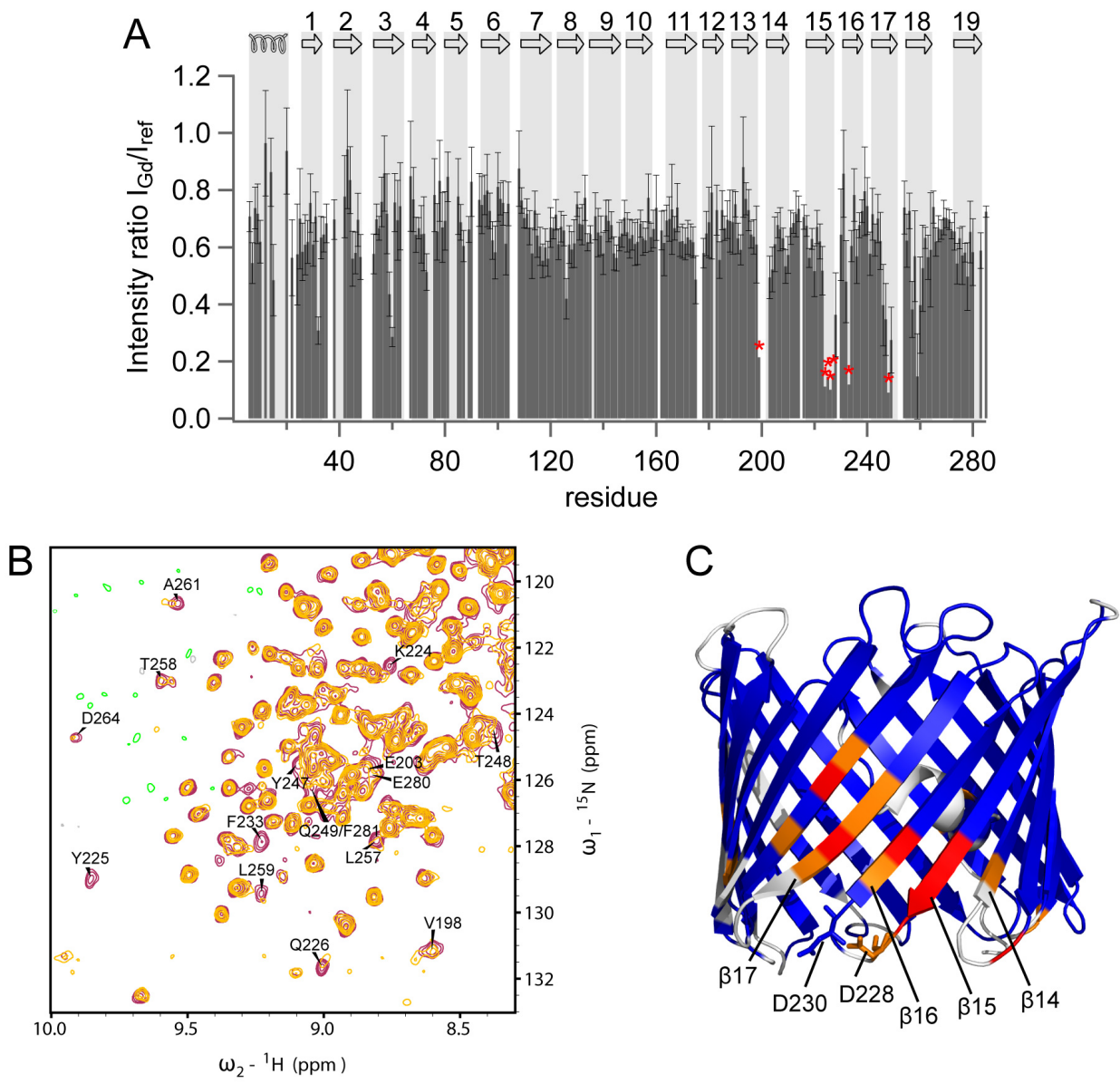

Figure 41. $\mathrm{Gd}^{3+}$ interaction confirms the $\mathrm{C}$-terminal $\mathrm{Ca}^{2+}$ binding site in hVDAC1. (A) Intensity ratios of hVDAC1 resonances in ${ }^{1} \mathrm{H},{ }^{15} \mathrm{~N}$-TROSY spectra in the presence $\left(1: 0.25\right.$ protein: $\left.\mathrm{Gd}^{3+}\right)$ and absence of $\mathrm{GdCl}_{3}$ (spectra were recorded by Monika Bayrhuber). Red stars indicate residues broadened beyond detection, where the noise level was used as peak intensities. The topology of hVDAC1 is shown on top with secondary structure elements highlighted in grey. (B) Region of ${ }^{1} \mathrm{H},{ }^{15} \mathrm{~N}-\mathrm{TROSY}$ spectra of hVDAC1 before (red) and after (yellow) addition of $\mathrm{GdCl}_{3} .(\mathrm{C})$ Residues broadened by more than $50 \%$ (orange) or beyond detection (red) are mapped onto the crystal structure of mVDAC1 (PDB code: 3EMN). Non-assigned or non-observable residues are coloured white.

\subsubsection{Interaction with ruthenium red (RuR) confirms $\mathrm{Ca}^{2+}$ interacting regions}

Another alternative for probing of calcium binding sites is the dye ruthenium red (RuR), which binds to $\mathrm{Ca}^{2+}$-binding sites of other proteins (Hadad et al. 1994; Reed and Bygrave 1974), induces channel closure of $w t$ VDAC1 reconstituted in bilayers, and protects cells from apoptosis (Israelson et al. 2008). Furthermore, $\mathrm{CaCl}_{2}$ competes with the binding of azo ruthenium red, a photoreactive ruthenium compound, and E73 and E203 have been implicated in binding of RuR as well (Israelson et al. 2007). A titration with RuR induced small chemical shift and intensity changes in similar regions in the $\mathrm{N}$ - and C-terminus (Figure 42). 


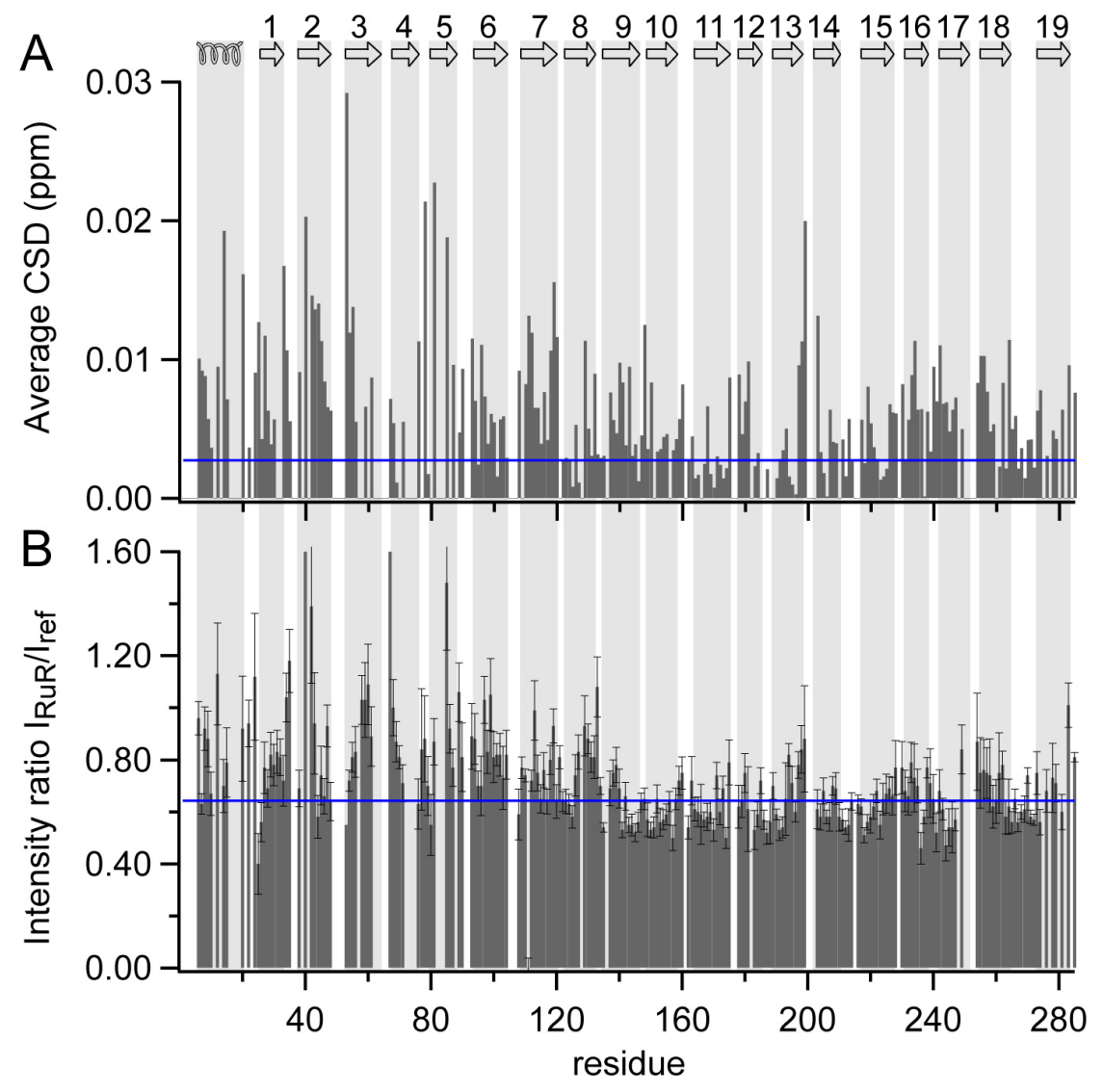

Figure 42. RuR interacts with $\mathrm{N}$ - and $\mathrm{C}$-terminal barrel regions of hVDAC1. (A) Average chemical shift deviations and of hVDAC1 resonances in ${ }^{1} \mathrm{H},{ }^{15} \mathrm{~N}$-TROSY spectra upon addition of RuR (hVDAC1:RuR 1:4). The blue line indicates the average CSD of non-shifting resonances. (B) Intensity changes after $\mathrm{RuR}$ addition (value \pm standard error). The blue line indicates the average resonance intensity for residues 120-280.

Small shifts were observed in the $\alpha$-helix, the linker, in an extensive region in the N-terminal $\beta$-strands $(\beta 1-\beta 9)$, and in the $\mathrm{C}$-terminal $\beta$-strands $\beta 15-\beta 18$, indicating weak binding of RuR to hVDAC1. These regions contain all regions that were influenced by $\mathrm{Ca}^{2+}$ and $\mathrm{Gd}^{3+}$. Additionally, shifts were observed in L11-12 and L13-14, which did not occur in the case of small ions. Intensity ratios changed in a complex manner upon RuR addition (Figure 42B). While the overall intensity was reduced, the resonance intensities of the same residues that exhibited RuR-induced shifts were unchanged, increased, or decreased only slightly. This result suggests a stabilization of residues in the $\mathrm{N}$ - and C-terminus by a reduction in $\mu$ s-ms dynamics upon RuR interaction.

Taken together, the interaction with $\mathrm{Ca}^{2+}, \mathrm{Gd}^{3+}$ and $\mathrm{RuR}$ locates the calcium interaction sites to two regions in the $\mathrm{N}$ - and C-terminal barrel regions of hVDAC1: 
One region is located in the N-terminal five $\beta$-strands. It includes E73 and overlaps with the strongly dynamic $\beta$-barrel region (see section 3.3). A second region is located towards one barrel site in the C-terminal $\beta 15-\beta 18$, which is also dynamic and prone to age-dependent aggregation. The latter region might also involve the linker. In contrast to the C-terminal ATP binding site, however, the linker is located further away, suggesting that the linker might form a third independent $\mathrm{Ca}^{2+}$ interaction region.

\subsubsection{4 $\mathrm{Ca}^{2+}$ influence on VDAC conductivity}

A previous study showed that $\mathrm{Ca}^{2+}$ influences VDAC gating, opening the channels for ATP transport at $100 \mu \mathrm{M}$ concentrations, while sub-micromolar concentrations or chelation of the divalent ion by EGTA reversibly closed the channels (Bathori et al. 2006). In a contradictory study neither EGTA $(0.4 \mathrm{mM})$ nor $\mathrm{Ca}^{2+}(1.6 \mathrm{mM})$ induced changes in VDAC gating (Rostovtseva et al. 2005). In order to investigate an influence of $\mathrm{Ca}^{2+}$ on the conductivity of our hVDAC1 preparations, we performed BLM measurements of single channel conductance in the absence and presence of $\mathrm{Ca}^{2+}$ and EGTA. In the absence of either substance, the major conductance steps were observed at 0.5 and $0.6 \mathrm{nS}$ in $0.1 \mathrm{M} \mathrm{KCl}$ (Figure 43). The usual conductance at this ionic concenctration is $0.45 \mathrm{nS}$ (Benz 1994). Thus, the observed values correspond to the open state and a state of slightly increased conductance, respectively. In the presence of EGTA these were still the most frequently occupied states (Figure 43). Additionally, smaller conductance steps $(0.2,0.3$, and $0.4 \mathrm{nS})$, corresponding to various closed states, were more frequently occupied (Figure 43). The change in conductivity might be a result of partial or transient channel closure by $\mathrm{Ca}^{2+}$ chelating. In agreement with the effects of EGTA on resonance shifts and intensities in the C-terminal ATP binding region (Figure 40), however, they might also reflect EGTA interaction in the pore lumen that leads to a reduction in ion flux. In the presence of $\mathrm{Ca}^{2+}$ the conductance step of $0.2 \mathrm{nS}$ was increasingly populated, while the conductance of $0.4 \mathrm{nS}$ was less populated (Figure 43). Furthermore, higher conductance steps (0.8 and $0.9 \mathrm{nS}$, and higher values) were more frequently observed (Figure 43), corresponding to the simultaneous insertion of several channels. However, due to the low number of insertions recorded, the statistics are not optimal. 


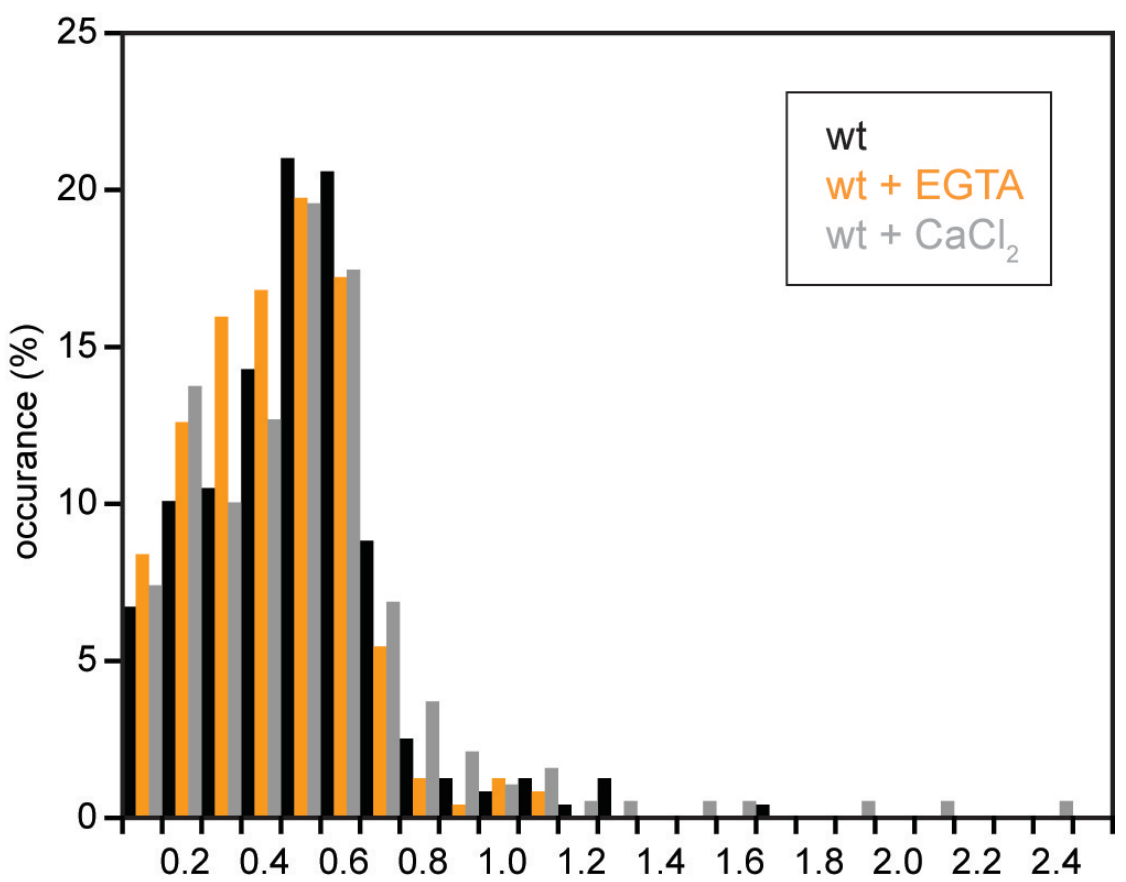

Figure 43. Conductance changes in the presence of Ca and EGTA. $w t$ hVDAC1 single channel BLM measurements were performed in the presence of $0.1 \mathrm{M} \mathrm{KCl}$ and $20 \mathrm{mM}$ Hepes $\mathrm{pH} 7.2(\mathrm{wt}, \mathrm{n}=238$ ). In separate experiments either $5 \mathrm{mM}$ EGTA (wt + EGTA, $\mathrm{n}=238)$ or $1 \mathrm{mM} \mathrm{CaCl}_{2}\left(\mathrm{wt}+\mathrm{CaCl}_{2}, \mathrm{n}=189\right)$ were included in the buffer.

Thus, in the presence of $\mathrm{Ca}^{2+}$ at best a slight tendency for insertion of highconductance, oligomeric hVDAC1 channels and changes the distribution of closed states occurred. These results suggest a role for $\mathrm{Ca}^{2+}$ on hVDAC1 oligomerization and gating, although the effects were not as drastic as previously published (Bathori et al. 2006). Higher $\mathrm{Ca}^{2+}$ concentrations and larger statistical analyses might improve the significance of the BLM measurements.

\subsubsection{Influence of $\mathrm{Ca}^{2+}$ interaction on hydrogen-bonds in hVDAC1}

Changes in the position and intensities of hVDAC1 resonances in the presence of $\mathrm{Ca}^{2+}$ and defined resonance broadening in the presence of $\mathrm{Gd}^{3+}$ locate the $\mathrm{Ca}^{2+}$ binding sites to regions in the $\mathrm{N}$ - and $\mathrm{C}$-terminal part of the hVDAC1 $\beta$-barrel. At the same time, the observation that a larger part of the N-terminal barrel region and the linker were affected by $\mathrm{CaCl}_{2}$ than the immediate binding site identified by $\mathrm{Gd}^{3+}$, indicates the presence of additional $\mathrm{Ca}^{2+}$-induced effects. These might be structural changes of the channel or modulation of $\mu \mathrm{s}-\mathrm{ms}$ dynamics, which were observed in the $\alpha$-helix, the linker, and in the $\mathrm{N}$ - and C-terminal $\beta$-strands of hVDAC1 (Figure 22 and Figure 24). Therefore, we analyzed $\mathrm{H}_{\mathrm{N}}$ temperature coefficients of $w t \mathrm{hVDACl}$ from a series of 
${ }^{1} \mathrm{H},{ }^{15} \mathrm{~N}$-TROSY spectra in the absence and presence of $5 \mathrm{mM} \mathrm{CaCl}_{2}$ (Figure 44). Temperature coefficients report on the sensitivity of hydrogen bonds on the external perturbation temperature. In the absence of $\mathrm{Ca}^{2+}$, strongly negative ${ }^{1} \mathrm{H}_{\mathrm{N}}$ temperature coefficients were observed in the $\alpha$-helix of hVDAC1 (Figure 45) in agreement with observations that temperature coefficients in $\alpha$-helices are on average $1 \mathrm{ppb} / \mathrm{K}$ more negative than in $\beta$-sheets (Cierpicki and Otlewski 2001). The majority of hVDAC1 residues in $\beta$-strands exhibited negative temperature coefficients with average values of approximately $-2.5 \mathrm{ppb} / \mathrm{K}$ for individual strands as expected for hydrogen-bonded residues in $\beta$-strands (Figure 45) (Cierpicki and Otlewski 2001). $\beta 1$ showed a slightly less negative average value of $-1.1 \mathrm{ppb} / \mathrm{K}$ (Figure 45B). In addition, $\beta 16$ exhibited many unusual positive values averaging to $-0.1 \mathrm{ppb} / \mathrm{K}$. These data show a pronounced temperature sensitivity of hydrogen bonds in $\beta 16$ and to a minor extent also in $\beta 1$. Among other factors that influence ${ }^{1} \mathrm{H}_{\mathrm{N}}$ temperature coefficients (e.g. the presence of aromatic ring currents), a weak correlation between the average length (and thereby inverse strength) of hydrogen bonds and temperature coefficients has been observed (Cierpicki and Otlewski 2001).
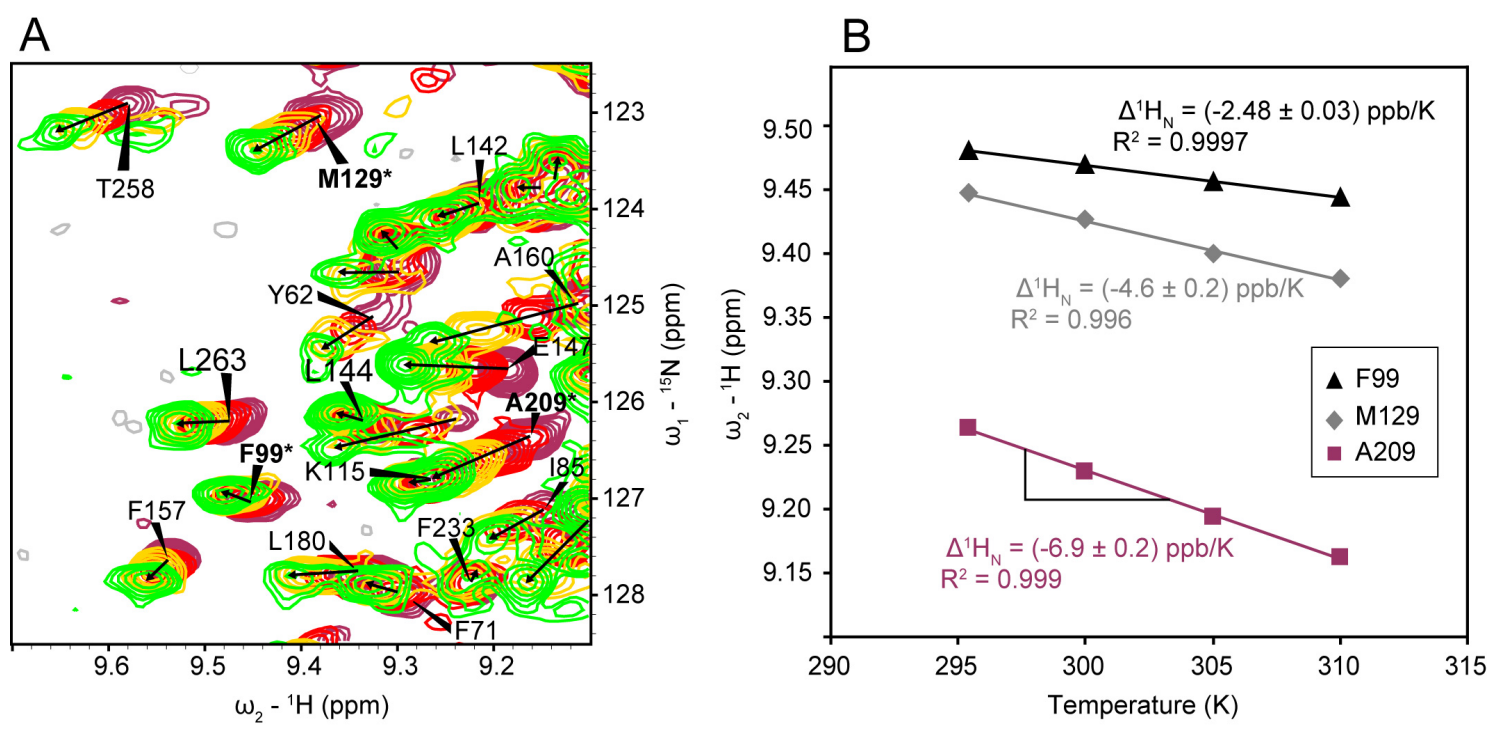

Figure 44. Determination of temperature coefficients. (A) Region of ${ }^{1} \mathrm{H},{ }^{15} \mathrm{~N}$-TROSY spectra recorded on $w t^{2} \mathrm{H} /{ }^{15} \mathrm{~N}$-hVDAC1 in the absence of $\mathrm{Ca}^{2+}$ at 295 (green), 300 (yellow), 305 (red), and $310 \mathrm{~K}$ (dark red). Temperature dependent resonance shifts are indicated by arrows. Some residue assignments are given. (B) ${ }^{1} \mathrm{H}_{\mathrm{N}}$ resonance positions of residues marked in bold and with an asterisk in (A) are plotted versus the temperature. The data points were fit by linear regression analysis, yielding ${ }^{1} \mathrm{H}_{\mathrm{N}}$ temperature coefficients $\left(\Delta^{1} \mathrm{H}_{\mathrm{N}}\right)$ from the slope (indicated exemplarily for A209). 

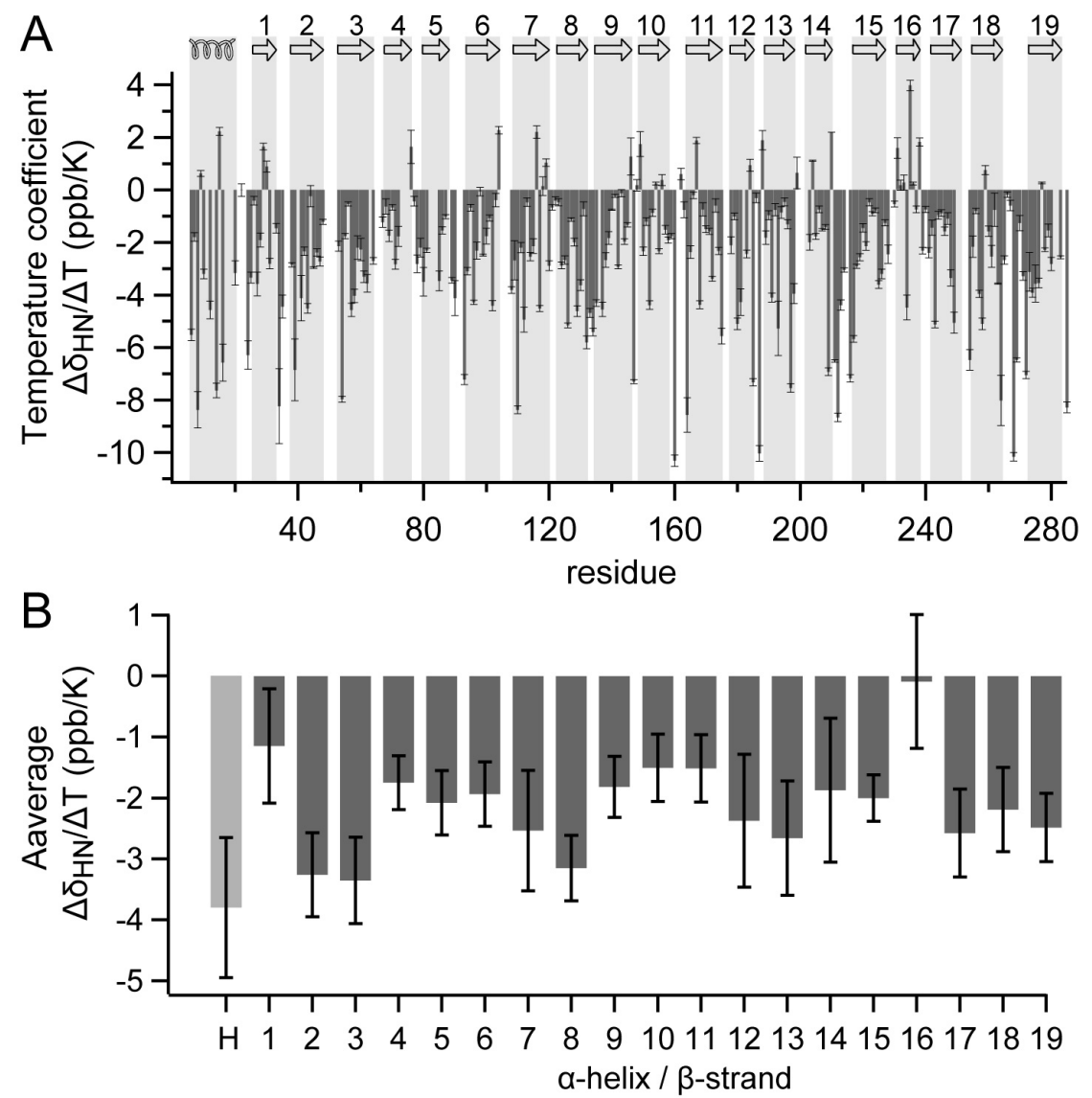

Figure 45. Temperature coefficients of hVDAC1. (A) Amide proton temperature coefficients $\Delta \delta_{\mathrm{HN}} / \Delta \mathrm{T}$ extracted from ${ }^{1} \mathrm{H},{ }^{15} \mathrm{~N}$-TROSY spectra measured at a ${ }^{1} \mathrm{H}$ frequency of $900 \mathrm{MHz}$ at 295.4, 300, 305, and $310 \mathrm{~K}$ (value \pm standard error). The topology of VDAC1 is indicated on top, with secondary structure elements highlighted in grey. (B) Average temperature coefficients for the $\alpha$-helix (light grey, $\mathrm{H}$ ) and strands $\beta 1-\beta 19$ (dark grey, 1-19) (mean \pm standard error).

In line with the increased H/D exchange of $\beta 1$ (Figure 20), the increased ${ }^{1} \mathrm{H}_{\mathrm{N}}$ temperature coefficients in $\beta 1$ indicate that hydrogen bonds are weaker in the parallel arrangement of $\beta 1$ and $\beta 19$. The increased ${ }^{1} \mathrm{H}_{\mathrm{N}}$ temperature coefficients in $\beta 16$ might reflect a weakening of hydrogen bonds. However, H/D exchange showed no sign of hydrogen bond breakage (Figure 20B). Furthermore, ${ }^{1} \mathrm{H}_{\mathrm{N}}$ temperature coefficients might be influenced by other factors, such as the three adjacent aromatic side chains Y225 $(\beta 15)$, F233 ( $\beta 16)$, and Y247 ( $\beta 17)$.

In the presence of $5 \mathrm{mM} \mathrm{CaCl}$ a trend towards higher (less negative) ${ }^{1} \mathrm{H}_{\mathrm{N}}$ temperature coefficients was observed for all strands except $\beta 16$ that showed slightly reduced values (Figure 46A). However, in most cases these differences were within the standard error and the alternative analysis of ${ }^{15} \mathrm{~N}_{\mathrm{H}}$ temperature coefficients did not reveal significant changes for either strand (Figure 46B). 


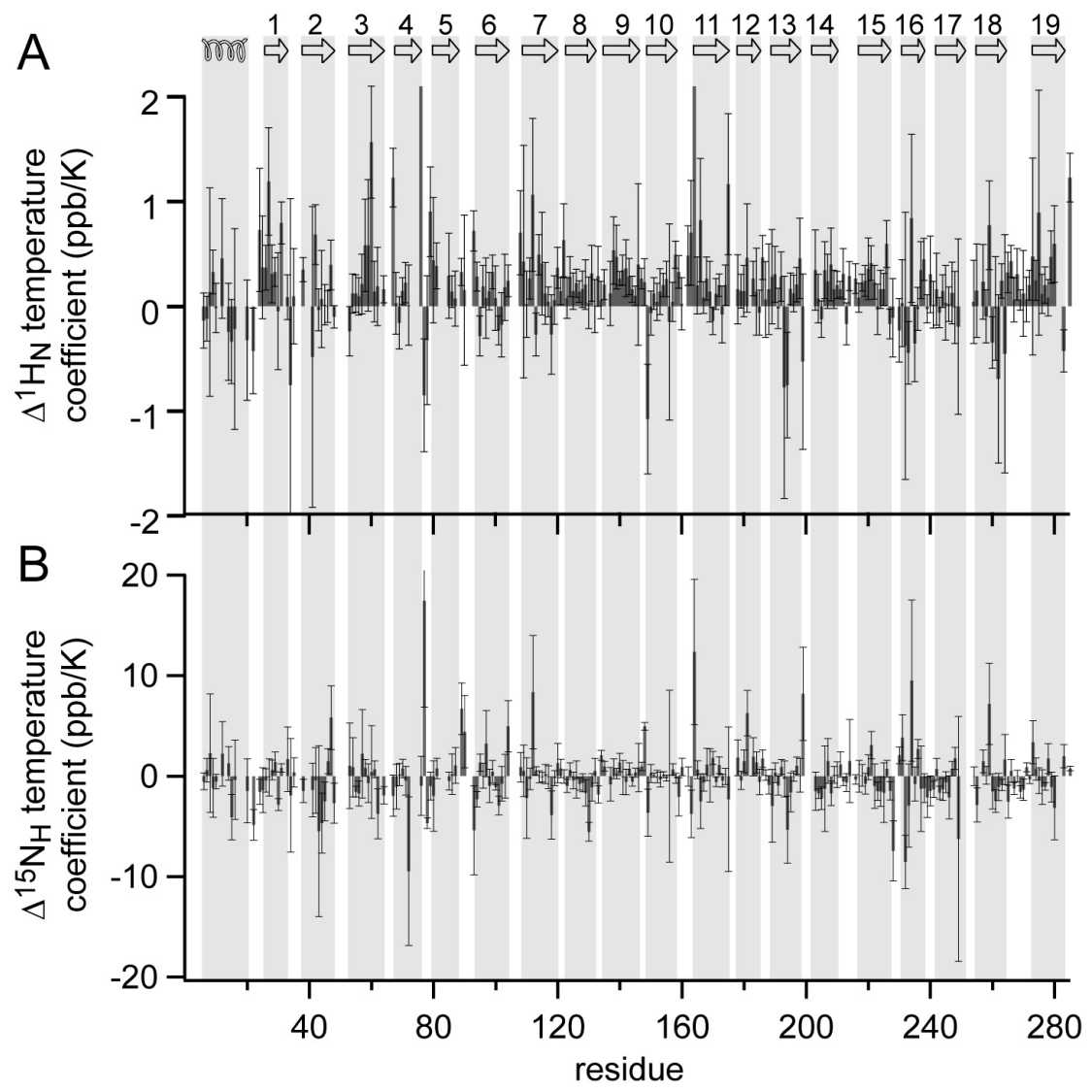

Figure 46. $\mathrm{Ca}^{2+}$ has a negligible influence on temperature coefficients of hVDAC1. (A) Change of amide proton $\left(\Delta^{1} \mathrm{H}_{\mathrm{N}}\right)$ temperature coefficients extracted from ${ }^{1} \mathrm{H},{ }^{15} \mathrm{~N}$-TROSY spectra measured at a ${ }^{1} \mathrm{H}$ frequency of $900 \mathrm{MHz}$ after addition of $5 \mathrm{mM} \mathrm{CaCl}_{2}$ (compared to the $\mathrm{Ca}^{2+}$-free state) (value \pm standard error). For clarity, the scale was cut above $\pm 2 \mathrm{ppb} / \mathrm{K}$. (B) Change of amide nitrogen $\left(\Delta^{15} \mathrm{~N}_{\mathrm{H}}\right)$ temperature coefficients for the same conditions as in (A) (value \pm standard error). The topology of VDAC1 is indicated on top, with secondary structure elements highlighted in grey.

Hence, $\mathrm{Ca}^{2+}$ binding has at best a very weak influence on the properties of the hydrogen bonds of the N-terminal strands and of $\beta 16$ in hVDAC1 when solubilized in LDAO micelles.

\subsubsection{6 $\mathrm{Ca}^{2+}$ and oligomerization of $\mathrm{hVDAC}$}

An alternative reason for the observed resonance broadening in $\beta 2-\beta 3$ might be $\mathrm{Ca}^{2+}$ induced oligomerization of hVDAC1 that is intermediate on the NMR time scale. As mentioned before, the strong broadening in the N-terminal barrel region mostly remained upon dilution of hVDAC1 in the absence of added $\mathrm{Ca}^{2+}$ (Figure 22). However, 6-fold dilution induced an overall intensity increase by a factor of 1.28 on average, with slightly larger intensity changes (by a factor of 1.16 compared to the overall increase) in the $N$-terminal barrel region, including $\beta$-strands and loops in the region $\beta 2-\beta 5$, and 
loop L5-6 (Figure 29C). This increase was accompanied by a reduction in rotational correlation time by $7 \mathrm{~ns}$ (Figure 29B). Furthermore, chemical shifts occurred both in the N-terminal ( $\beta 2-\beta 7)$ and the C-terminal ( $\beta 14-\beta 18)$ barrel regions of hVDAC1 (Figure 47). The strongest shifts (Figure 47) and intensity changes (Figure 29C) upon dilution in the N-terminal barrel region and loops were observed in loop L1-2 (S35), $\beta 2$ (S43, S44, A47), $\beta 3$ (K53, V54, G56, K61, Y62, W64), $\beta 4$ (Y67, G68, F71), loop L4-5 (N79, T80), $\beta 5$ (I85, V87), loop L5-6 (D89-A92), $\beta 6$ (K96, F103), and $\beta 7$ (Y118, R120). In the $\mathrm{C}$-terminal part, the strongest effects were located in $\beta 11$ (T165, Q166), $\beta 12$ (F178), $\beta 13$ (E189), $\beta 14$ (L208), $\beta 15$ (Y225), $\beta 16$ (D230, F233, S234), loop L16-17 (S240, S241), $\beta 17$ (Y247), loop L17-18 (G254, I255), $\beta 18$ (L259), and the very C-terminus (S285). Although dilution affected a broader region than $\mathrm{Ca}^{2+}$, both the N-terminal $(\beta 1$ $\beta 5)$ and $\mathrm{C}$-terminal $(\beta 15-\beta 18) \mathrm{Ca}^{2+}$ interaction sites are contained within these regions. Thus, excluding the linker, the same regions that interact with $\mathrm{Ca}^{2+}$ are also involved in dynamic oligomerization of hVDAC1 when solubilized in LDAO micelles. Although some residues shifted in the same direction upon $\mathrm{Ca}^{2+}$ addition and increasing concentration, a clear correlation was not observed (data not shown).

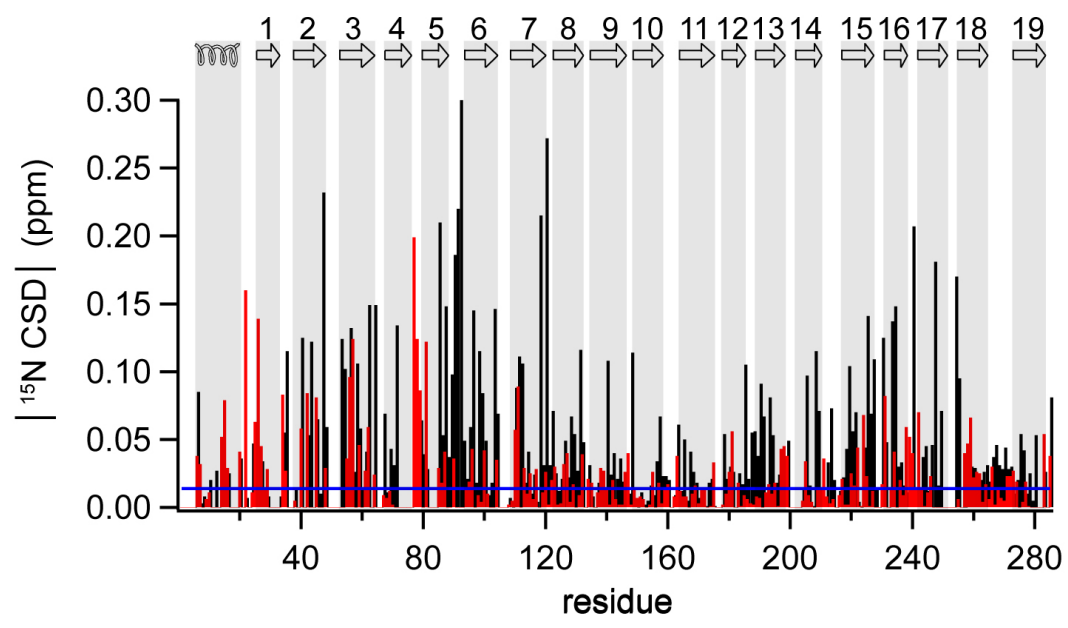

Figure 47. Oligomerization sites overlap with $\mathrm{Ca}^{2+}$ binding sites of hVDAC1. Magnitude of ${ }^{15} \mathrm{~N}$ chemical shift deviations (CSD) observed in $2 \mathrm{D}{ }^{1} \mathrm{H},{ }^{15} \mathrm{~N}$-TROSY spectra of hVDAC1 upon 6-fold dilution of hVDAC1 (black). The magnitude of ${ }^{15} \mathrm{~N}$ CSD induced by a 16-fold $\mathrm{Ca}^{2+}$ excess (as in Figure 39A) is shown in red. The blue line indicates the average ${ }^{15} \mathrm{~N}$ CSD of non-shifting residues. The topology of $\mathrm{hVDAC1}$ is indicated on top with secondary structure elements highlighted in grey. 


\section{Discussion}

\subsection{The structure of $\mathrm{hVDAC} 1$ represents the native conformation}

The physiological significance of the 19-stranded $\beta$-barrel structures of mammalian VDAC1 was questioned, because of the applied refolding protocols and inconsistent biochemical data (Colombini 2009). Support for the physiological significance of the 19 -stranded $\beta$-barrel is provided by the successful and fast incorporation of refolded $\mathrm{hVDAC} 1 / \mathrm{mVDAC} 1$ in bilayer membranes in this (Figure 10) and other studies (Hiller et al. 2008; Ujwal et al. 2008). The channel displayed typical open and closed state conductance and voltage-dependent closure. Membrane insertion of VDAC (time scale of several minutes) is considerably faster than OmpA insertion from an unfolded intermediate (several hours) (Kleinschmidt et al. 1999; Tamm et al. 2004). In contrast, the fast and functional incorporation of solubilized VDAC into bilayers indicates a nearnative structure in micelles (Benz 1994). Refolding into native structures is also successfully applied to bacterial OMPs, even the larger channels with large domains within the pore (in-plugs) (Buchanan 1999; Pautsch and Schulz 1998; Schmid et al. 1998). In order to match the biochemical data, a rearrangement of the VDAC $\beta$-strands was proposed to occur during or after membrane insertion of refolded micellar VDAC1. However, the proposed rearrangement involves motion of six $\beta$-strands out of the membrane and rotation of three $\beta$-strands (Colombini 2009). This requires disruption of approximately 72-90 hydrogen-bonds (8-10 hydrogen-bonds per strand) and is incompatible with the spontaneous membrane incorporation of (h)VDAC1. Thus, we conclude that recombinantly expressed and refolded hVDAC1 adopts the native structure with most of the hydrogen bonds preformed. 


\subsection{Dynamics in the hVDAC1 barrel on a wide time scale}

Increasing evidence suggests that conformational changes in VDAC are important for (voltage-dependent) gating and induction of apoptosis (as reviewed in (ShoshanBarmatz et al. 2010a)). ${ }^{6}$ Large conformational changes during gating have long been proposed by electron microscopy (Guo and Mannella 1993) and bilayer measurements (Peng et al. 1992; Zimmerberg and Parsegian 1986). Many charged residues in the $\beta$ barrel and the $\alpha$-helix were proposed to form a mobile "voltage-sensor" involved in gating motions (Song et al. 1998a; Thomas et al. 1993). Recent studies propose that these motions involve a reorientation of the N-terminal $\alpha$-helix into the pore lumen (Hiller and Wagner 2009; Ujwal et al. 2008). The modulation of VDAC channel conductance by small molecules and apoptotic proteins in vivo (Shoshan-Barmatz et al. 2006) strongly implicates VDAC as a regulator of metabolism and apoptosis (Lemasters and Holmuhamedov 2006; Shoshan-Barmatz et al. 2008).

By a combination of solution NMR spectroscopy, Gaussian network model analysis and molecular dynamics simulation we showed that (i) hydrogen bonds in the N-terminal three $\beta$-strands of hVDAC1 are transiently broken (Figure 20), (ii) $\mu$ s-ms dynamics are significantly increased for the N-terminal six $\beta$-strands (Figure 21 and Figure 24), (iii) a charge on the membrane-facing E73 plays a key role for the elevation of N-terminal protein dynamics (Figure 30), (iv) mutation of E73 reduces hydrogen bond instability (Figure 20), (v) mutation and chemical modification of E73 strongly reduce $\mu$ s-ms dynamics in solution (Figure 27 and Figure 28), and (vi) charge on the side chain of E73 facing the membrane in the $\beta$-barrel is coupled to thinning of the nearby membrane (Figure 31 ).

The dynamics of VDAC1 presented in this study set the protein apart from bacterial $\beta$-barrel membrane proteins. While for bacterial OMPs loops play a major role in immunogenicity, gating or catalysis (Arora et al. 2001; Hong et al. 2006; Hwang and Kay 2005; Liang et al. 2010; Liang and Tamm 2007; Renault et al. 2009; Schulz 2000;

\footnotetext{
${ }^{6}$ The entire section 4.2 (including subsections) is a part of our own recent publication (Villinger et al. 2010) and contains identical text passages.
} 
Yildiz et al. 2006), our study reveals a unique involvement of a large area of the $\beta$ barrel in functional dynamics of a eukaryotic membrane $\beta$-barrel protein.

NMR spectroscopy revealed conformational exchange, which occurs in the Nterminal $\beta$-strands $\beta 2-\beta 7$ of the hVDAC1 barrel on the time scale of $\mu \mathrm{s}-\mathrm{ms}$ (Figure 21 and Figure 24), in addition to the conformational flexibility observed in $\beta 1-\beta 3$ (Figure 20). In addition to slow dynamics, increased ps-ns flexibility in LDAO-solubilized hVDAC1 was detected for strands $\beta 2$ and $\beta 3$ by NMR spectroscopy (Figure 23). Since the MD simulations of VDAC1 embedded in lipid bilayers were performed for simulation times up to $\sim 150 \mathrm{~ns}$, it can probe protein dynamics that occur on the ns time scale. B-factors in crystal structures have been found to correlate well with NMR derived $S^{2}$ order parameters reporting on ps-ns dynamics (Clore and Schwieters 2006; Yang et al. 2009). In addition, static disorder contributions to B-factors may reflect slower dynamics when multiple conformations are present in the crystal. In extreme cases of conformational exchange, electron densities are often not refined (e.g. in CitA (Sevvana et al. 2008)), such that possible correlations of B-factors and slow dynamics are invisible. In case of the N-terminal region of mVDAC1, contributions to B-factors might arise from dynamics and static disorder intermediate in amplitude such that crystal structure refinement was possible. Together the data indicate the presence of extensive protein dynamics in the N-terminal part of the VDAC1 $\beta$-barrel on a wide range of time scales. The flexibility of the N-terminal part of the VDAC1 $\beta$-barrel is increased on time scales above $1 \mathrm{~ns}$ in the MD simulations (Figure 30) and the correlation with crystallographic B-factors was best for the lowest frequency GNM modes (Figure 26). Furthermore, strong chemical exchange in the $\mu \mathrm{s}-\mathrm{ms}$ time scale and H/D exchange on longer time scales was observed by NMR spectroscopy (Figure 21 and Figure 24). Thus, slow collective motions are likely to play a key role. Part of these slow collective motions could be the global deformation of the VDAC1 $\beta$-barrel that was observed in the MD simulations (Figure 30). Experimental information about these slow motions might also be gained from Carr-Purcell-Meiboom-Gill (GPMG) NMR relaxation dispersion experiments (Loria et al. 1999a), which can be used to characterize the structure of low-populated excited states that are invisible with other techniques. Although a TROSY-CPMG version for large molecules exists (Loria et al. 
1999b), the very high molecular weight in case of the VDAC-micelle complex likely exceeds the limit for recording high quality data required to draw reliable conclusions about the structure of the excited state(s). An indication for the structure of the excited state comes from the second conformation of S57 ( $\beta 3)$ in the 3D TROSY-HNCA spectrum. The $\mathrm{C} \alpha$ chemical shift value of the second conformation of S57 was shifted by $\sim 0.2 \mathrm{ppm}$ towards the random coil value (Figure 25), suggesting that the N-terminal barrel region is less structured in the minor conformation. This assumption is in line with a higher disorder probability predicted in $\beta 1-\beta 2$ in this work (Figure 20B) and in a recent study (Geula et al. 2011).

\subsubsection{The role of charge on E73 for VDAC1 dynamics}

Our study demonstrates that the dynamics in the $\beta$-barrel of hVDAC1 are mainly determined by the membrane-facing residue E73. While the protonation state of E73 is unclear in our micellar solution as well as in the crystal structure obtained in a bicellar environment, MD simulations clearly show that a charge on the side chain of E73 has the ability to increase motion in the N-terminal barrel region and to reduce the membrane thickness next to E73 (Figure 31). Other functionally relevant glutamic and aspartic acids, which are located in hydrophobic pockets of proteins, have $\mathrm{pK}_{\mathrm{a}}$ values as high as 8 (Valiyaveetil et al. 2002). Assuming a high $\mathrm{pK}_{\mathrm{a}}$ value for VDAC as well, VDAC is partially protonated in solution at the $\mathrm{pH}$ used here (6.8). DCCD inhibits the channel activity of VDAC1 in planar lipid bilayers in a voltage-dependent manner, requiring incubation at high negative or positive potentials. In addition, DCCD slows down the transition from the high-conducting to a long-lived low-conducting state by stabilizing intermediate states of the VDAC1 channel (Shafir et al. 1998). The occupation of long-lived intermediate states is in agreement with the reduction in intrinsic dynamics by DCCD modification reported here. However, one should note that the time scale of intermediate state stabilization is in the range of minutes (Shafir et al. 1998) and thus considerably larger than the micro-to-millisecond exchange observed by NMR.

We conclude that the decreased protein dynamics observed in the $\mathrm{N}$-terminus of hVDAC1 upon mutation of E73 to valine and by chemical modification with DCCD is caused by removal of the partial charge. Although E73 has a strong influence on the 
stability of VDAC1, the faster H/D exchange in $\beta 2$ persistent in E73V hVDAC1 (Figure 20A) suggests that other residues in the N-terminal barrel region might as well play a role for barrel dynamics. These could include S43, predicted as the most unstable residue in this $\beta$-strand (Geula et al. 2011).

\subsubsection{Functional aspects of VDAC1 dynamics}

Our observations have important implications for VDAC1's function as a gatekeeper for metabolites and its role in apoptosis. Residues suggested to be involved in gating of Saccharomyces cerevisiae VDAC (scVDAC) are located in the N-terminal $\alpha$-helix, the linker, as well as the first five $\beta$-strands (Thomas et al. 1993). E73, located within this region, is conserved in human VDAC isoform 2 (hVDAC2) and in mammals (Supporting figure 3). Mutation of E73 to glutamine (E73Q) shows reduced voltagedependence of gating and inhibits RuR- and hexokinase-mediated effects on channel function and protection from apoptosis (Zaid et al. 2005). The observed effects were attributed to inefficient binding of hexokinase to the mutated form of VDAC (AbuHamad et al. 2008). Moreover, the reaction of hVDAC1 with DCCD abolishes the interaction of VDAC with hexokinase (Nakashima et al. 1986; Zaid et al. 2005). Thus, our results imply that dynamics in the N-terminal region are relevant for gating and hexokinase binding. This interpretation is in line with recent findings showing destabilized regions of outer membrane proteins to be preferentially involved in proteinprotein interaction (Naveed et al. 2009). In addition, deviation from a circular structure determines the selectivity of maltoporin LamB (Meyer et al. 1997; Schirmer et al. 1995) and sucrose-specific porin ScrY (Forst et al. 1998). Thus, the observed deformation in the N-terminal strands of VDAC1 (Figure 30) might also determine VDAC selectivity and gating by a dynamic change between various deformed states.

MD simulations of mVDAC1 showed that the nature and charge status of a single residue, in this case E73, can be highly coupled to the global motion of a protein. This result is remarkable, as E73 is not located anywhere near a hinge region but roughly in the middle of a barrel-forming $\beta$-strand. At the same time the charge and polarity of E73 is strongly correlated with the dynamics and conformation of the membrane environment, suggesting that the dynamics of the lipids might be connected with global protein motions. This is an interesting finding, as lipid-protein interactions 
are known to be important for the structure and function of integral membrane proteins (Bogdanov et al. 2010; Galdiero et al. 2007; Hunte and Richers 2008; Kagan and Thundimadathil 2010; Langosch and Arkin 2009; Nyholm et al. 2007). In particular, VDAC1 gating and ionic selectivity have been shown to be dependent on lipid composition (Rostovtseva and Bezrukov 2008). The MD simulations of mVDAC1 in a lipid bilayer demonstrated that a charge on E73 causes a perturbation of the surrounding membrane (Figure 30). Since the major lipid components of outer mitochondrial membranes are zwitterionic lipids (phosphatidylcholine and phosphatidylethanolamine) (de Kroon et al. 1997), the membrane perturbation is likely to occur also in vivo and might relate to non-specific membrane rupture proposed to occur during apoptosis (Feldmann et al. 2000; Rostovtseva et al. 2005). 


\subsection{Structure and dynamics of the N-terminal helix in hVDAC1}

Apart from the $\beta$-barrel, a second region in VDAC is in the focus of our research: the Nterminal $\alpha$-helix. Previous studies proposed a location of the VDAC $\alpha$-helix either on the membrane surface (Guo et al. 1995; Mannella 1998) or accessible to the cytoplasm (De Pinto and Palmieri 1992). Additionally, residues in the $\alpha$-helix have been suggested to be involved in voltage-gating (Thomas et al. 1993), during which helix motion was proposed to occur (Hiller and Wagner 2009; Mannella 1997; Ujwal et al. 2008). Furthermore, the $\alpha$-helix was found to interact with antibodies (Abu-Hamad et al. 2006; Junankar et al. 1995), Bcl-2 (Shi et al. 2003) and hexokinase (Abu-Hamad et al. 2009), which might involve a detachment of the $\alpha$-helix from the $\beta$-barrel. Notably, the three high-resolution structures of mammalian VDAC1 disagree mainly in the position and orientation of the $\alpha$-helix (Bayrhuber et al. 2008; Hiller et al. 2008; Ujwal et al. 2008).

\subsubsection{Ambivalence of the $\mathbf{N}$-terminal helix: barrel stabilization and dynamics}

While elevated dynamics in the N-terminal $\beta$-barrel region were clearly observed by various methods and in different hydrophobic environments (see above), the data for the $\alpha$-helix is less well-defined. On one hand, we observed (i) an agreement of resonance frequencies of the N-terminal $\alpha$-helix up to residue V17 in micelles and liposomes (Table 5) and a kink around residue 9-11 (Figure 13), (ii) an influence of the R15A mutation on adjacent barrel residues (Figure 15), (iii) a reasonable fit of RDC values for helical residues with the crystal structure of mVDAC1 obtained from bicelles (Ujwal et al. 2008) (Figure 19 and Table 6), and (iv) low crystallographic B-factors (Figure 26), low ENM-predicted residue fluctuations (Figure 26), high resonance intensities (Figure 21) and low exchange rates (Figure 24) in the central $\beta$-strands. These data strongly suggest a kinked structure of the $\mathrm{N}$-terminal $\alpha$-helix similar to the crystal structure of mVDAC1 (Ujwal et al. 2008) and a stabilization of the central $\beta$-strands by interaction with the $\alpha$-helix. Thus, the chemical shift and intensity changes observed for R15A hVDAC1 in the distant N-terminal strands (Figure 15) might be explained by an influence of the $\alpha$-helix rigidity on the overall stability of hVDAC1. This assumption is 
in line with instability of $\beta 9$ observed when the complete helix is removed from hVDAC1 in liposomes (Schneider et al. 2010). Although truncation of the helix does not lead to a complete unfolding in solution, a destabilization or aggregation occurs as well (unpublished observations).

On the other hand, we observed (i) missing C $\alpha$ connections and NOEs (Figure 12), (ii) potential second resonance sets for the linker (G21-G25) (Figure 14), (iii) a reduction of signal intensities towards the second part of the helix (Figure 21), (iv) fast H/D exchange for residues V17 and K20 (Figure 20), and (v) elevated $\mu$ s-ms dynamics for residues A14, R15, and D16 in the second helical part (Figure 24). These data suggest the presence of conformational exchange in the $\alpha$-helix/linker in micellar solution. Admittedly, the lack of assignments and distance restraints in this region can be caused by signal overlap. Moreover, the absence of restraints does not prove the presence of dynamics and is the most obvious reason for the disorder of residues 11-20 in the pure NMR structure (Hiller 2008) and for the different $\alpha$-helical structure in our combined NMR/X-ray structure (Bayrhuber et al. 2008) with respect to the crystal structure of mVDAC1 (Ujwal et al. 2008). Fast H/D exchange in $\alpha$-helices is considered to arise from unfolding of the entire secondary structure element (Englander and Kallenbach 1983), suggesting some degree of unfolding for the C-terminal end of the $\alpha$-helix. However, the increased H/D exchange can also be related to elevated solvent access and does not necessarily indicate dynamics. In contrast, the amide resonance broadening and the elevated ${ }^{15} \mathrm{~N}$ relaxation rates are clear indications for conformational exchange. Considering dynamics and a possible unfolding of the second helical segment, the second possible explanation for the effects in the N-terminal barrel region in R15A hVDAC1 is the existence of a low populated state, in which the helix is in proximity to the $\mathrm{N}$-terminal barrel region.

Resonance broadening and ${ }^{15} \mathrm{~N}$ relaxation data indicate that the dynamics occur on the micro- to millisecond timescale, while elevated H/D exchange and second resonance sets for the linker suggest dynamics on slower time scales. These data are in contrast to the lack of any sign of sub-millisecond and slower dynamics in the $\alpha$-helix in solid state NMR of hVDAC1 in liposomes (Schneider et al. 2010). However, highly flexible regions cannot be observed in the performed carbon solid state spectra 
(DREAM, PDSD), but their dynamics might contribute to broadening of amide resonances in solution. Furthermore, the time scales of dynamics might be slightly different in the two environments. Moreover, resonance broadening of ${ }^{1} \mathrm{H}$ and relaxation data of ${ }^{15} \mathrm{~N}$ nuclei, in combination with smaller line widths in solution NMR, are very sensitive reporters for dynamics. The population of excited states is probably low and might exhibit $\mathrm{C} \alpha$ chemical shifts similar to the rigid state, such that the excited states can be hidden in solid state experiments.

In summary, we conclude that the main population of the helix is in a rigid kinked $\alpha$-helical structure, while low populated excited states with a different structure of the second $\alpha$-helical part are also present. Due to the missing resonance assignments and constraints for the second $\alpha$-helical part any assumption on the exact structure would be speculation.

\subsubsection{Cross-talk of dynamics in the $\beta$-barrel and the $\alpha$-helix}

Our data revealed two dynamic regions in hVDAC1: the N-terminal $\alpha$-helix and the Nterminal six $\beta$-strands. Moreover, a single destabilizing point mutation in the $\alpha$-helix at position 15 affects the entire $\beta$-barrel (Figure 15). Vice versa, the barrel stabilizing mutation E73V slightly increased signal intensities in the $\alpha$-helix (Figure 27A). These data suggest that the stability of the $\alpha$-helix and the $N$-terminal $\beta$-barrel region are interconnected. Residues in both the $\alpha$-helix and the $\mathrm{N}$-terminal $\beta$-barrel region have been implicated in voltage-gating (Thomas et al. 1993). At the same time, hexokinase binding involves both the N-terminus and the $\alpha$-helix (Abu-Hamad et al. 2009; AbuHamad et al. 2008; Azoulay-Zohar et al. 2004). Thus, our data connects the two regions important for interactions and voltage-gating via dynamics. The mobility of the $\alpha$-helix might be transferred to the $\mathrm{N}$-terminal barrel region and vice versa by the flexible linker.

\subsubsection{Models for VDAC1 gating involving the $\alpha$-helix}

The helix dynamics revealed in our study have important implications for interactions and gating of hVDAC1. Although the $\alpha$-helix is involved in stabilization of the barrel most of the time, the observed mobility in the second part of the $\alpha$-helix might be 
required for voltage-gating or protein interactions as suggested previously (Abu-Hamad et al. 2009; Hiller and Wagner 2009; Ujwal et al. 2008). Since the first part of the $\alpha$ helix is attached to the barrel in all three high-resolution structures and the dynamics mainly occur in the second part of the $\alpha$-helix, our data fit to the gating model suggested by Wagner et al. (Figure 48A). In that model, stimuli such as NADH, induce subsequent displacement of the linker and the second $\alpha$-helical part from the barrel wall. In addition, gating might involve unfolding of the $\alpha$-helix, such that it can approach the N-terminal region (Figure 48B). The resulting steric and electrostatic blockage of the pore lumen could result in the reduced ion conductivity and abolished metabolite transport. However, the reduction of small ion conductance to $20-50 \%$ as observed in bilayer measurements (Benz 1994; Schein et al. 1976) might require further motion of the $\beta$-barrel. In line with this suggestion, Poisson-Boltzmann calculations are unable to reproduce the experimental selectivity change for a hypothetical closed state obtained by motion of the complete helix into the pore (Choudhary et al. 2010). The barrel deformations around E73 observed by MD simulations of hVDAC1 in lipid bilayers (Figure 30) could facilitate an interaction of the helix with the opposite barrel wall. By this mechanism the amplitude of the helix motion required for channel closure could be reduced.
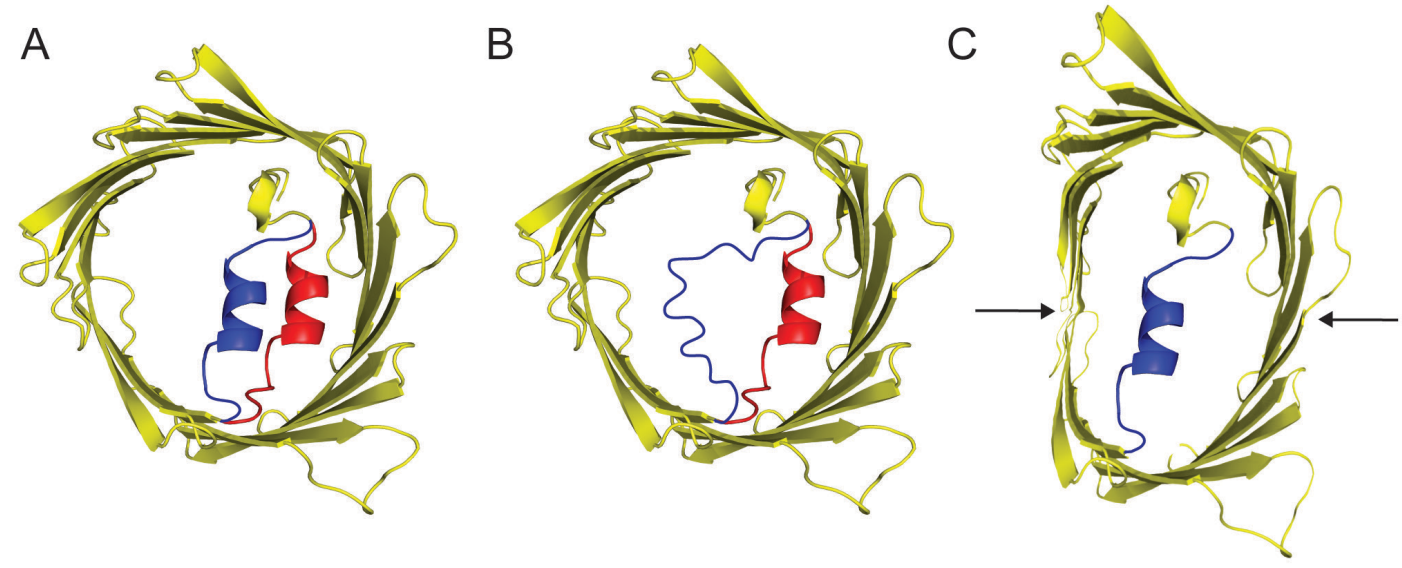

Figure 48. Gating models of VDAC1. The structure of mVDAC1 (PDB code: 3EMN) is shown as a ribbon representation in yellow with the second part of the helix and the linker (residues 13-25) coloured in red. Superimposed in blue is a displaced helical region as proposed (Hiller and Wagner 2009) (A) and the same region in an unstructured conformation (B). (C) Graphical model of barrel deformation upon displacement of the second helical region (blue). 
Furthermore, detachment of the second $\alpha$-helical part from the $\beta$-barrel is expected to induce destabilization of the C-terminal barrel region. This will lead to a further barrel destabilization than what is already induced by the strong dynamics in the $\mathrm{N}$-terminal $\beta$-barrel region. Consequently, this might lead to an increased barrel deformation or even a collapse of the barrel (Figure 48C). A barrel destabilization by removal of the helix could explain the channel closure of truncated VDAC forms in bilayer experiments (De Pinto et al. 2008; Koppel et al. 1998; Popp et al. 1996). Alternatively, the $\beta$-barrel might collapse without detachment of the $\alpha$-helix, facilitated by the dynamics in the N-terminal part of the $\beta$-barrel (see section 4.2) and the second part of the $\alpha$-helix.

The flexibility in the second part of the N-terminal $\alpha$-helix in hVDAC1 distinguishes the channel from bacterial OMPs. The N-terminal $\alpha$-helix of hVDAC1 resembles the long loop L3 in bacterial OMPs (Bayrhuber et al. 2008; Zeth and Thein 2010). In these membrane proteins a large displacement of loop L3 during voltagegating was initially proposed and later excluded by cross-linking L3 to the adjacent $\beta$ barrel (Bainbridge et al. 1998; Eppens et al. 1997), suggesting that voltage-gating is a result of "multiple subtle rearrangements" of charged residues (Phale et al. 1997). Furthermore, relatively low B-factors indicate (semi-) rigidity of L3 in other porins (Schmid et al. 1998; Weiss and Schulz 1992) and (helical) in-plugs are frequently used to stabilize flexible barrel regions in various bacterial OMPs (Naveed et al. 2009). The observed dynamics in the $\mathrm{N}$-terminal $\beta$-barrel region and the second part of the $\alpha$-helix in hVDAC1 already present in the absence of any closure inducing stimuli (voltage, small molecules, or proteins) provides an explanation for the low voltages sufficient for closure of the mitochondrial porin $(20-30 \mathrm{mV})$, in contrast to the voltages of $\sim 100 \mathrm{mV}$ required for gating of bacterial porins (Liu and Delcour 1998; Saxena et al. 1999; Schulz 2000). 


\subsection{Nucleotide interactions of hVDAC1}

The main function of VDAC is the regulated transport of metabolites (mainly ATP and ADP) across the OMM (Benz 1994; Rostovtseva and Colombini 1997) and specific nucleotide interactions have been indicated by several studies (Rostovtseva and Bezrukov 1998; Yehezkel et al. 2007; Yehezkel et al. 2006). Interactions with $\mathrm{Ca}^{2+}$ are proposed to open the channel for ATP/ADP exchange (Bathori et al. 2006), providing a connection between mitochondrial $\mathrm{Ca}^{2+}$-signalling and energy metabolism. We have probed the interaction sites for nucleotides and $\mathrm{Ca}^{2+}$ in hVDAC1 and discuss our findings in the light of VDACs role in metabolite transport, gating, oligomerisation and apoptosis.

\subsubsection{VDAC possesses a nucleotide binding site encompassing the helix-to- barrel linker}

We have shown that hVDAC1 possesses a C-terminal ( $\alpha$-helix, linker, $\beta 12-19$, Figure 33 ) and an N-terminal ( $\beta 1-\beta 8$ including the GLK motif, Figure 34 and Figure 35 ) nucleotide binding site. In a preliminary study on the interaction of VDAC with ATP performed by G. Wagner and co-workers a few residues potentially involved in ATP binding were identified. These residues included F24 and G25 in the linker region, I114 and T116 from the second NBS and D264 and A283 in the third NBS (Malia 2006). However, by focussing only at chemical shift changes, Wagner and co-workers concluded that ATP binding to hVDAC1 is unspecific (Hiller et al. 2008). Here we identified - on the basis of a more complete resonance assignment and including the effects on signal intensities (Figure 32C) - consecutive stretches of interacting residues in the C-terminal barrel part that provide a consistent picture of the ATP binding sites in hVDAC1. In addition, we estimated the affinity for uncomplexed ATP with a dissociation constant in the low millimolar range (Figure 36).

The identified ATP binding sites are in agreement with previously identified NBS in rat VDAC1 (rVDAC1) natively purified from mitochondria or in intact mitochondria (Yehezkel et al. 2006), strengthening the indications that the recombinantly expressed protein adopts a 3D structure similar to the natively open 
form. According to the combined NMR/X-ray structure of hVDAC1 (Bayrhuber et al. 2008) and the crystal structure of the highly homologues mVDAC1 (Ujwal et al. 2008), NBS1 and NBS3 form a single binding site (the C-terminal ATP binding site). Within this binding region, a partial Walker B motif $\left({ }^{260}\right.$ SALLD $\left.^{264}\right)$ in the C-terminal strands and an inverted Walker A motif $\left({ }^{19} \mathrm{TKGYGFG}^{25}\right)$ in the linker region have been discussed as putative nucleotide binding sites (Hiller et al. 2008; Walker et al. 1982; Yehezkel et al. 2006; Zizi et al. 1994). A similar Walker B motif is present in the adenenine nucleotide translocase (ANT) of the inner mitochondrial membrane (Zizi et al. 1994). Notably, the inverted Walker A motif alone binds ATP as a short peptide (Florke et al. 1994). In general, lysine residues have been shown to be involved in ATP binding (Yehezkel et al. 2006). In addition, mutant forms of scVDAC (homologous residues in hVDAC1) influencing channel selectivity often involve lysine or arginine residues: K19 (K20), K46 (S46), K61 (K61), K65 (T65), K84 (E84), K95 (K96), R124 (G126), K234 (S234), K248 (T248), T256 (K256) (Blachly-Dyson et al. 1990; Peng et al. 1992) (Figure 49). However, one should note that only five out of these ten residues are positively charged in hVDAC1, with many of them not located near the C-terminal ATP binding site. However, the NMR studies demonstrated that K20, G21, and G23 are located in the C-terminal ATP binding site. Mutation of these residues changes channel properties, ATP transport and mitochondrial metabolism of mVDAC1, with K20 having the largest effect (Yehezkel et al. 2007) (Figure 49). K20 is therefore most likely involved in binding of the negatively charged phosphate moiety of ATP. Similarly, MD simulations revealed that phosphate binding to the anion selective porin OprP involves several lysine residues (Pongprayoon et al. 2009).

K236, another positively charged residue in the C-terminal ATP binding site of VDAC1, might contribute to phosphate binding via generation of an additional positive electrostatic potential. However, this case is complicated by the fact that K234 and K236 have been shown to be required for membrane insertion of scVDAC in vitro and in vivo, with a more pronounced effect for the K234 mutant (Angeles et al. 1999; Smith et al. 1995). In hVDAC1 and mVDAC1 K234 is replaced with a serine residue, suggesting that membrane insertion of the mammalian channel might have different requirements. 


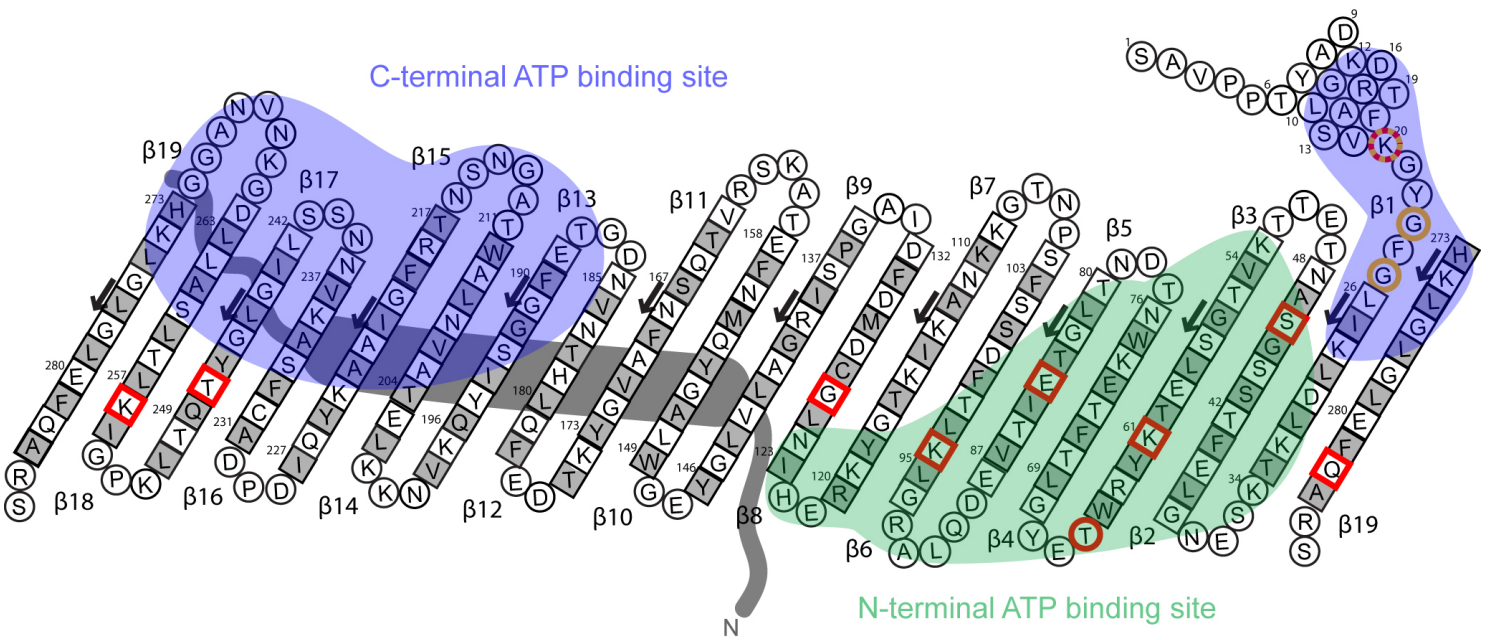

Figure 49. Residues influencing selectivity and ATP transport. Topology map of hVDAC1 (adapted from (Bayrhuber et al. 2008)). Boxed residues that form $\beta$-strands with outward pointing residues are coloured in grey. Encircled residues are located in loops or the N-terminal $\alpha$-helix. Boxed/encircled in red are residues at positions homologous to lysine or arginine residues in scVDAC, which influence selectivity (Blachly-Dyson et al. 1990; Peng et al. 1992). Residues that change channel properties, ATP transport and mitochondrial metabolism (Yehezkel et al. 2007) are encircled in orange. Grey tubes in the background indicate the approximate position of the N-terminal $\alpha$-helix and linker in the hVDAC1 structure. The approximate locations of the $\mathrm{C}$ - and N-terminal ATP binding sites are indicated in blue and green, respectively.

The location of S234 (K234 in scVDAC) and K236 close to the linker region (Figure 49) indicates that these residues might be required for stabilization of the helix/linker and proper folding of the channel. Indeed, K236 forms a hydrogen bond (or cation- $\pi$ interaction (Gromiha and Suwa 2007)) to F18 in mVDAC1 (Ujwal et al. 2008), thus providing a connection between helix structure/position and ATP binding. Further studies are required to investigate the role of charged interactions between the phosphate moiety with K20 or K236, as well as specific interactions with the adenosine group.

\subsubsection{Nonspecific ionic influences in the N-terminal barrel half of VDAC}

An N-terminal ATP-binding site was identified in $\beta$-strands $\beta 1-\beta 9$, comprising the conserved glycine-leucine-lysine $\left({ }^{94} \mathrm{GLK}^{96}\right)$ motif in $\beta 6$ that contains K96 (Figure 49). The GLK motif is close to $\beta 7$ and therefore likely identical to NBS2 found by photoaffinity labelling (Yehezkel et al. 2006). The nearby residue K61 is a candidate for the involvement in ATP binding. The involvement of K61 and K96 in ion selectivity of scVDAC and Neurospora crassa VDAC (ncVDAC) has been demonstrated by bilayer measurements (Blachly-Dyson et al. 1990; Runke et al. 2000) (Figure 49) and 
electrostatic calculations (Choudhary et al. 2010). However, the observed ATP induced changes in signal position and intensity are scattered over a large region (Figure 35), which is in large part also influenced by $\mathrm{NaCl}$ (Figure 37) and $\mathrm{CaCl}_{2}$ (Figure 39). Furthermore, the N-terminal region is implicated in binding of cations (Choudhary et al. 2010; Rui et al. 2011). All ionic species will sense the electrostatic potential of the pore lumen and affect the electrical environment of charged residues in the N-terminal barrel region, many of them responsible for selectivity and voltage-gating (Blachly-Dyson et al. 1990; Thomas et al. 1993). However, binding of ATP differs from monovalent ion interactions in that it requires several specific interactions. We therefore attribute the observed effects in the $\mathrm{N}$-terminal region to a distribution of the nucleotide and counter ions in the channel lumen without tight ATP binding. Therefore, the estimated $\mathrm{K}_{\mathrm{D}}$ for this region reflects an average affinity for ATP and small ions.

\subsubsection{Affinity, selectivity, and transport of ATP}

The observation that $\mathrm{Na}_{2} \mathrm{ATP}$ induces much stronger changes in hVDAC1 than MgATP (Figure 32) indicates that the charged species $\mathrm{ATP}^{4-}$ - or $\mathrm{HATP}^{3-}$ or both - interacts with VDAC. Nucleotides induce current noise in bilayer measurements of VDAC, which was attributed to a direct nucleotide binding (Rostovtseva et al. 2002). Noise generation increases with the charge of the molecule $\left(\mathrm{ATP}^{4-}>\mathrm{ADP}^{3-}>\mathrm{AMP}^{2-}\right)$, agreeing with a stronger binding of negatively charged metabolites to VDAC (Rostovtseva et al. 2002). Support for the binding of the charged form also comes from inhibition of photoaffinity ATP labelling by divalent ions (Yehezkel et al. 2006). The affinity of hVDAC1 for ATP (estimated $K_{D} \sim 1 \mathrm{mM}$ ) is about two orders of magnitude higher than previously suggested from ATP flux studies in bilayers $\left(\mathrm{K}_{\mathrm{D}} \sim 75 \mathrm{mM}\right)$ (Rostovtseva and Colombini 1997). Considering a possible underestimation of the NMR-derived dissociation constant by up to two orders of magnitude due to intermediate exchange contributions (Feeney et al. 1979), our value is in agreement with previous reports. However, physiological concentrations of ATP in the millimolar range (3.3 mM cytosolic ATP, $8 \mathrm{mM}$ ATP in the matrix, (Traut 1994)) make a $\mathrm{K}_{\mathrm{D}}$ value in the lower millimolar range appear more reasonable.

The higher affinity of hVDAC1 for the uncomplexed ATP species raises the question: How is ATP transported by VDAC? In reconstituted bilayers permeation of 
less negatively charged molecules such as succinate ${ }^{2-}$ and citrate ${ }^{3-}$ is favoured over $\mathrm{ATP}^{4-}$ permeation (Rostovtseva and Colombini 1997). This suggests that in vivo ATP is transported by VDAC as the less charged $\mathrm{Mg}^{2+}$-complex, which is the predominant physiological form (Akerboom et al. 1978; Traut 1994) and the form most kinases, including hexokinase (Darby and Trayer 1983), require for catalysis. On the other hand,

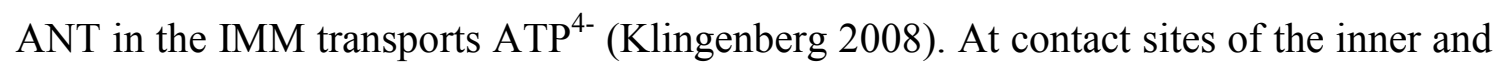
outer mitochondrial membrane (composed of VDAC-ANT complexes (Brdiczka 1991)) the transport of the charged form might prevail.

Further insight into metabolite transport can be gained by comparison with bacterial porins. Increasing evidence suggests that selectivity and transport requires specific solute binding (Dutzler et al. 1996; Lou et al. 2011; Meyer and Schulz 1997; Nestorovich et al. 2002; Pongprayoon et al. 2009; Schirmer et al. 1995; Zachariae et al. 2008; Zou et al. 2008). For example, the selective OprP discriminates between organic phosphate and $\mathrm{Cl}^{-}$ions by specific interactions with lysine residues (Pongprayoon et al. 2009), while a single lysine mutation inhibits phosphate flux (Sukhan and Hancock 1996). Further examples are interactions of antibiotics with the bacterial porin OmpF (Nestorovich et al. 2002) and antibiotic resistance caused by single mutations in OmpC that destroy the transversal electrostatic potential in the pore, while leaving the pore size unchanged (Lou et al. 2011). This suggests that the picture of porins as simple "molecular sieves", discriminating solutes by molecular weight, is oversimplified. VDAC also discriminates between physiological and non-physiological charged molecules of similar size (Rostovtseva et al. 2002). Furthermore, solute affinities of hVDAC1 $\left(\mathrm{K}_{\mathrm{D}}\right.$ in the low millimolar range) and bacterial OMPs are similar (Meyer et al. 1997; Moraes et al. 2007; Pongprayoon et al. 2009; Zachariae et al. 2006). The low affinity of all porins might enable passive transport, because it is sufficient for discrimination of transported molecules, while at the same time it enables release of transported molecules. In line with this proposal, a diffusion model of ion transport describes flux maximization by specific interactions, the strength of which is adjusted to physiological metabolite concentrations (Berezhkovskii and Bezrukov 2005). Thus, we conclude that interaction of VDAC with the charged form of ATP can be a mechanism of exerting selectivity and accelerating transport of this species at the same time. However, this does not exclude the transport of the $\mathrm{Mg}^{2+}$-complex of ATP. 
This model also suggests that the impermeability of the closed VDAC for ATP (Perevoshchikova et al. 2010; Rostovtseva and Colombini 1997) might not be purely related to a steric blockage of the channel, for instance by motion of the helix into the pore (Hiller and Wagner 2009; Ujwal et al. 2008), a strong barrel deformation (see section 4.2) or both. Instead, it could be an effect of side chain and backbone rearrangements that strongly reduce the affinity for ATP and thereby abolish ATP transport.

Since the C- and N-terminal ATP binding sites are located towards opposite pore entrances (Figure 49), a slight difference in affinity might result in ATP shuttling from the intermembrane space (probably N-terminal ATP binding region) to the cytosol (probably C-terminal binding region). Such a shuttling mechanism resembles the transport of phosphate through OprP via two binding sites (Pongprayoon et al. 2009) or the smooth gliding of substrates through maltoporin by optimally spaced nonpolar and polar interactions (Meyer and Schulz 1997; Schirmer et al. 1995). A more detailed understanding of metabolite transport might be gained from MD simulations with different ATP complexes.

\subsubsection{VDAC possesses a common nucleotide binding site}

GTP and UTP induced changes in NMR signal positions and intensities for the same residues as ATP (Figure 38). In addition, the ATP/GTP/UTP-binding site partially overlaps with regions of $\beta$-NADH binding, for which the binding-hotspots are localized at residues 242-244 ( $\beta 17)$ and 260-264 ( $\beta 18)$ (Hiller et al. 2008). Together the data point to a common nucleotide binding site in VDAC1 that allows transport of different metabolites. On the other hand, the presence of NADH affects a smaller number of residues (Hiller et al. 2008) when compared to ATP (Figure 32). In addition, ATP but not NADH can release VDAC from reactive red agarose (Yehezkel et al. 2006), suggesting distinct differences in binding interfaces. Discrimination of nucleotides by hVDAC1 might be realised by differences in binding interfaces or affinities according to the above-mentioned model of solute selectivity and transport. The affinity of $\mathrm{hVDAC1}$ for ATP $\left(\mathrm{K}_{\mathrm{D}}\right.$ in the low millimolar range) is well below that for NADH $\left(\mathrm{K}_{\mathrm{D}} \sim\right.$ $16 \mu \mathrm{M}$ (Zizi et al. 1994) to $87 \mu \mathrm{M}$ (Lee et al. 1994)). NADH enhances voltage gating of VDAC (Zizi et al. 1994) and reduces the channel's permeability to ADP, which has 
been proposed to contribute to the Crabtree effect (inhibition of oxidative phosphorylation at high glucose levels) in metabolic regulation (Lee et al. 1994). Interaction of NADH with VDAC might require a higher affinity, in order to fulfil its regulatory function and further reflects the lower physiological concentration of this nucleotide in the cytosol $(0.27 \mathrm{mM})$ (Tischler et al. 1977). The proximity of the NADH and ATP binding sites suggests that NADH inhibits ATP/ADP transport by competing with the same binding site rather than inducing structural changes in the $\alpha$-helix and linker as suggested (Ujwal et al. 2008).

\subsubsection{Implications of ATP binding for VDAC gating}

In contrast to NADH, a regulatory function of ATP itself has not been proven to date. At near-physiological ATP concentrations $(5 \mathrm{mM})$ no effect on VDAC gating was observed in bilayers (Zizi et al. 1994). The transport of large amounts of ATP across the mitochondrial membrane probably requires a lower affinity of VDAC for this nucleotide, which is in line with the absence of an apparent regulatory effect of ATP. However, ATP binding to VDAC inverts the channel's selectivity (Runke et al. 2000) and has been suggested to block the channel sterically (Rostovtseva and Bezrukov 1998; Rostovtseva et al. 2002). Alternatively, the observed NMR resonance broadening (Figure 32C) might originate in part from conformational exchange that occurs on the time scale of micro-to-milliseconds. In line with this proposal, Rostovtesva et al. admitted that the ATP-induced noise generation observed for VDAC might also result from fluctuations in the protein structure (Rostovtseva et al. 2002). Moreover, residue $\mathrm{K} 20$ in the helix/linker has been proposed to be involved in gating motions (Thomas et al. 1993) and the second $\alpha$-helical part and the linker might adopt alternative conformations. In addition, the linker is located between the side chains of S260 and L262 in the crystal structure of mVDAC1 (Ujwal et al. 2008), suggesting that conformational changes in the linker are required, if ATP-binding envokes the complete ${ }^{260}$ SALLD $^{264}$ motif (Figure 49). Similarly, it has been suggested that NADH binding displaces the $\alpha$-helix and thereby leads to channel closure (Ujwal et al. 2008). Such an ATP induced channel closure could explain how respiration rates are coupled to cellular energy requirements. 


\subsubsection{VDAC-ATP-hexokinase: a model}

Hexokinase induces VDAC channel closure and prevents apoptosis (Azoulay-Zohar et al. 2004). Both hexokinase- and voltage-induced channel closure prevent ATP flux through VDAC in mitochondria or bilayers, respectively (Perevoshchikova et al. 2010; Rostovtseva and Colombini 1997). However, the hexokinase induced VDAC closure fails to explain the exclusive supply of the kinase with ATP from the mitochondrial matrix (Gots et al. 1972; McCabe 1994). A possible mechanism that might explain this paradox is that hexokinase induced channel closure is actually a steric blockage of the VDAC channel from the cytosolic face. Thus, ATP diffusion into the channel from the IMS would still be possible, followed by binding to hVDAC1 and relay to hexokinase.

An alternative mechanism might be deduced from the present study - i.e. identification of the binding site that includes the $\alpha$-helix/linker and the $\beta$-barrel, as well as potential conformational changes induced by ATP binding in this region. The Nterminal $\alpha$-helix of VDAC1 is important for hexokinase interaction (Abu-Hamad et al. 2009). Under native conditions hexokinase interaction might detach the $\mathrm{N}$-terminal VDAC helix from the $\beta$-barrel. Such a detachment will destabilize the barrel as suggested by our data (see e.g. sections 4.2 and 4.3). If ATP interaction stabilizes the attachment of the $\alpha$-helix to the $\beta$-barrel by involving residues in the barrel wall and the $\alpha$-helix/linker, this interaction might compete with hexokinase induced helix detachment, prevent VDAC closure and maintain the ATP supply of hexokinase in a tight regulation. In line with this mechanism it was hypothesized that ATP binding transiently opens VDAC in the hexokinase-VDAC complex (McCabe 1994). Since $\mathrm{S} / \mathrm{K} 234$ and K236 are important for membrane insertion in vivo (Angeles et al. 1999; Smith et al. 1995), ATP interaction with these residues in the C-terminal ATP binding region links ATP binding with folding and barrel stabilization. 


\section{5 $\quad \mathrm{Ca}^{2+}$ interaction of VDAC}

\subsubsection{VDAC possesses two $\mathrm{Ca}^{2+}$ binding sites}

By the use of NMR titrations with $\mathrm{CaCl}_{2}$ and $\mathrm{GdCl}_{3}$ we have shown that hVDAC1 has two binding sites for $\mathrm{Ca}^{2+}$ : (i) An N-terminal site close to E73, observed from the calcium titration (Figure 39); (ii) a C-terminal site, where $\mathrm{Ca}^{2+}$ itself induces only small effects, but broadening by paramagnetic $\mathrm{Gd}^{3+}$ proves a direct interaction (Figure 41).

Two glutamic acids, E73 and E203, were suggested to be important for $\mathrm{Ca}^{2+}$ binding, since mutation of either glutamic acid abolished binding of RuR and azo ruthenium red (AzRu) to VDAC1 (Israelson et al. 2007; Israelson et al. 2008). RuR is known to bind to $\mathrm{Ca}^{2+}$-binding sites of other proteins (Hadad et al. 1994; Reed and Bygrave 1974) and $\mathrm{CaCl}_{2}$ competes with $\mathrm{AzRu}$ binding (Israelson et al. 2007). RuR induced chemical shift and intensity changes in similar regions in the $\mathrm{N}$ - and C-terminus (Figure 42), supporting the presence of both $\mathrm{Ca}^{2+}$ binding sites. In the original publication (Israelson et al. 2007), E73 and E203 were suggested to be in two cytosolic loops in close proximity. However, this was not supported by the recent threedimensional structure of VDAC1 (Bayrhuber et al. 2008; Hiller et al. 2008; Ujwal et al. 2008). E203 is located at the edge of the C-terminal binding site found by NMR and therefore appears not to be directly involved in $\mathrm{Ca}^{2+}$-binding. Instead, the $\mathrm{C}$-terminal binding site contains two aspartic acids at positions 228 and 230 presumably involved in the binding (Figure 39). MD simulations and Poisson-Boltzmann calculations indicate a preferred localization of monovalent cations in the $\mathrm{N}$-terminal region inside the pore (Choudhary et al. 2010; Rui et al. 2011). Thus, the effects of $\mathrm{CaCl}_{2}$ and $\mathrm{NaCl}$ in the $\mathrm{N}$ terminal $\beta$-strands around E73 observed in our study are in line with an interaction of cations with residues in the $\mathrm{N}$-terminal $\beta$-strands from the pore interior. However, the side chain of E73 itself points to the hydrophobic environment. Furthermore, the crystal structure of mVDAC1 displayed a $\mathrm{Ca}^{2+}$ ion located on the pore outside, complexed by the two E73 side chains in the asymmetric dimer (Ujwal et al. 2008). Thus, in micellar solutions $\mathrm{Ca}^{2+}$ might also bind to the $\mathrm{N}$-terminal region of the hVDAC1 barrel from the outside (hydrophobic environment) involving the charged side chain of E73. This might 
explain the modest effect of $\mathrm{Gd}^{3+}$ in the $\mathrm{N}$-terminal region. Due to the larger size and charge compared to $\mathrm{Ca}^{2+}, \mathrm{Gd}^{3+}$ appears not to be able to bind to the side chain of E73, pointing to the hydrophobic environment.

Taken together our data support the existence of two independent $\mathrm{Ca}^{2+}$-binding sites, one of them possibly involving $\mathrm{Ca}^{2+}$-binding from the channel outside. For transport of $\mathrm{Ca}^{2+}$ through the VDAC1 channel, binding inside the pore might be more important, as transport across the hydrophobic bilayer next to E73 is likely to be energetically disfavoured.

\subsubsection{Impact of $\mathrm{Ca}^{2+}$ on gating, stability, and oligomerization of hVDAC1}

$\mathrm{Ca}^{2+}$ and RuR induced only weak effects on hVDAC1 in solution. Thus, a large structural change resulting in the opening of VDAC from a completely closed pore (Bathori et al. 2006) is not evident from our NMR data. However, our bilayer experiments indicate a slight change towards high conductance states of hVDAC1 (Figure 43). Our data are thus in favour of a minor effect of $\mathrm{Ca}^{2+}$ on channel opening or stabilization of the open state, residing between the observations from two contradictory studies (Bathori et al. 2006; Rostovtseva et al. 2005). The absence of large effects might also be related to the presence of $\mathrm{Ca}^{2+}$ traces in our hVDAC preparations that would stabilize the channel in an open conformation throughout all NMR and BLM measurements. This is in agreement with the observed ATP binding in solution suggesting that hVDAC1 adopts an open state (Rostovtseva and Colombini 1997; Rostovtseva and Bezrukov 1998) without the necessity of $\mathrm{Ca}^{2+}$-addition.

However, $\mathrm{Ca}^{2+}$ - and RuR induced effects in the $\alpha$-helix, linker and the dynamic $\mathrm{N}$-terminal barrel region (Figure 39 and Figure 42). Since these region might play a role in VDAC gating (Figure 48 and (Hiller and Wagner 2009; Thomas et al. 1993; Ujwal et al. 2008)), our data support a $\mathrm{Ca}^{2+}$-induced structural change or stabilization of the open structure (Bathori et al. 2006). How this could be achieved, however, remains currently illusive. In contrast to ATP, it is unlikely that the significantly smaller $\mathrm{Ca}^{2+}$ could stabilize the linker-to-barrel contact, especially in light of the presence of positively charged residues (K20 and K236) in this region. Moreover, lanthanides and RuR induce VDAC channel closure in lipid bilayers at any applied voltage and prevent $\mathrm{Ca}^{2+}$ efflux 
from VDAC-containing liposomes (Gincel et al. 2001). The strong binding of $\mathrm{Gd}^{3+}$ in the C-terminal region thus suggests that this region is involved in channel closure. Consequently, the tendency of the C-terminal region for conformational exchange (Figure 21 and Figure 24) could be related to gating motions involving the $\beta$-barrel. Alternatively, lanthanides might close VDAC by changing the electrostatic potential in the pore lumen.

$\mathrm{Ca}^{2+}$ caused modest signal broadening and chemical shifts in $\beta$-strands around E73 (Figure 39A), a region that also displayed elevated H/D exchange and $\mu$ s-ms dynamics depending on the charge of the side chain of E73 (see section 4.2). Thus, complexation of a negatively charged E73 side chain with a $\mathrm{Ca}^{2+}$ ion - as observed in the crystal structure (Ujwal et al. 2008) and indicated by our NMR data - might have two consequences: (i) Reduction of the $\mu \mathrm{s}-\mathrm{ms}$ dynamics due to charge neutralization, similar to what was observed upon mutation or chemical modification of E73 (Figure 27 and Figure 28) and removal of the charge at the side chain of E73 in MD simulations (Figure 30); (ii) $\mathrm{Ca}^{2+}$-induced dimerization of hVDAC1 as suggested by the crystal structure of mVDAC1 (Ujwal et al. 2008). A reduction of $\mu$ s-ms dynamics would result in an increase in signal intensity, in contrast to what was observed in the presence of $\mathrm{Ca}^{2+}$. In addition, the presence of $\mathrm{Ca}^{2+}$ caused only minor changes in ${ }^{1} \mathrm{H}_{\mathrm{N}}$ temperature coefficients (Figure 46), while mutation of E73 to valine strongly reduced amide-proton exchange in the N-terminal part of the $\beta$-barrel (Figure 20). This suggests that $\mathrm{Ca}^{2+}$ induced oligomerization could be responsible for the observed signal broadening. Higher order oligomers (trimers, tetramers, hexamers, and higher oligomeric states) were previously observed in phospholipase A2 treated outer mitochondrial membranes (OMM) (Guo et al. 1995), native OMM (Hoogenboom et al. 2007; Mannella 1982), in whole mitochondria, and for purified VDAC (Zalk et al. 2005). Concentrationdependent changes in NMR signal positions and intensities (Figure 29 and Figure 47) thus suggest that one oligomerization interface of hVDAC1 is formed by the N-terminal $\beta$-strands $-\beta 18, \beta 19, \beta 1-\beta 4$ as found in the asymmetric dimer (Ujwal et al. 2009; Ujwal et al. 2008), or $\beta 18, \beta 19, \beta 1$ and $\beta 2$ as found in the symmetric dimer (Bayrhuber et al. 2008), or slightly shifted in the presence of $\mathrm{Ca}^{2+}$ binding to E73. A second interface required for the higher oligomers might then be formed by the C-terminal part of the hVDAC1 barrel. This assumption is supported by the finding that $\mathrm{Bcl}-\mathrm{x}_{\mathrm{L}}$ interacts 
with residues in $\beta$-strands $\beta 15-\beta 18$ and disrupts VDAC trimers (Hiller et al. 2008; Malia and Wagner 2007). Furthermore, strategic placement of cysteines at several positions in rVDAC1 indicate the presence of two oligomerization interfaces under physiological conditions: Firstly, $\beta$-strands $\beta 19, \beta 1$, and $\beta 2$; secondly, $\beta 16$ and $\beta 17$ (Geula et al. 2011). Notably, the second oligomerization site involves predicted unstable residues in $\beta 16(\mathrm{C} 232, \mathrm{~A} 231)$ and $\beta 17(\mathrm{Q} 249)$, which are in the centre of the strongest $\mathrm{Ca}^{2+}$ and $\mathrm{Gd}^{3+}$-effects (Figure 39 and Figure 41). This also indicates that the observed intensity reduction (Figure 21) and $\mu \mathrm{s}-\mathrm{ms}$ dynamics (Figure 24) in the Cterminal barrel region arise from dynamics and oligomerization in parallel. Moreover, a disulfide bridge formation via C232 was proposed by Shoshan-Barmatz et al. (Geula et al. 2011). Since $\mathrm{C} 232$ points into the pore interior, this process requires reorientation of the cysteine towards the lipid surrounding. Dimerization might further include complexation of $\mathrm{Ca}^{2+}$-ion by the loop residues D228 or D230 across monomers. As a consequence, in the presence of $\mathrm{Ca}^{2+}$ the interfaces might tighten and stabilize oligomers.

Physiological steady state $\mathrm{Ca}^{2+}$ concentrations in the cytosol are in the submicromolar range (Bathori et al. 2006) and are unlikely to explain the effects observed at the high concentrations (1-9.6 mM) used in this study. However, transient $\mathrm{Ca}^{2+}$ pulses with high local concentrations might be able to induce oligomerization. As both mitochondrial $\mathrm{Ca}^{2+}$ overload and oligomerization of VDAC1 have been related to the induction of apoptosis (Crompton 1999; Keinan et al. 2010; Shoshan-Barmatz et al. 2010b), our data suggest a possible connection of the two mechanisms. In particular, $\mathrm{Ca}^{2+}$ might influence dimer formation in the PTP (Szabo et al. 1993), although this pore complex also depends on the $\mathrm{Ca}^{2+}$ sensitivity of other pore constituents.

\subsubsection{Interplay of $\mathrm{Ca}^{2+}$ interaction, dynamics, and oligomerization}

The N-terminal $\beta$-strands $\beta 1-\beta 6$ and the $\mathrm{C}$-terminal strands $\beta 15-\beta 17$, are dynamic hot spots of hVDAC1: In these regions conformational dynamics occur on a wide time scale, and $\mathrm{Ca}^{2+}$ interaction and oligomerization/age-dependent aggregation takes place. The presence of dynamics, interactions and oligomerization in similar regions complicates the NMR analyses, but at the same time suggests that both regions are key regions for the channel's function. As pointed out above, complexation of a $\mathrm{Ca}^{2+}$ ion 
between two E73 side chains might occur in hVDAC1 in solution, when traces of the divalent ion are present. Consequently, homo-oligomerization might contribute to the exchange broadening in the N-terminal barrel region of $w t$ VDAC, while the major contribution of backbone dynamics to the exchange phenomena is strongly supported by H/D exchange and MD simulations. The reduced dynamics observed in E73V hVDAC1 might actually cause a reduction in dimerization tendency. Additionally, dynamics in $\beta 15-\beta 17$ overlap with and could facilitate oligomerization. This proposal is in line with the observation that dynamic regions in bacterial OMPs are prone to homo- and heterooligomerization (Naveed et al. 2009). 


\section{Conclusions and Perspectives}

In this study, NMR spectroscopy revealed functional dynamics of VDAC1 involving several regions. (i) The N-terminal $\beta$-strands $\beta 1-\beta 6$, influenced by a partially charged E73 that points to the hydrophobic environment; (ii) the second part of the N-terminal $\alpha$-helix and the linker connecting the helix to the first $\beta$-strand; (iii) the C-terminal $\beta$ strands $\beta 15-\beta 17$. The dynamics revealed here are important for protein and lipid interactions, gating, and oligomerization of VDAC, both separately and inter-connected. Our findings thus increase the insight on the role of the channel in regulating cellular energy metabolism and apoptosis. In addition, the location of the C-terminal ATP binding site, encompassing the $\alpha$-helix, linker, and adjacent $\beta$-strands $\beta 12-\beta 19$, indicates a connection to gating via nucleotide induced stabilization of the open state. The specific interactions with ATP provide an explanation for selectivity and regulated metabolite transport via the channel. Finally, $\mathrm{Ca}^{2+}$ interacts with two barrel regions $(\beta 1$ $\beta 5$ and $\beta 15-\beta 18)$ that are also involved in dynamic oligomerization, pointing to a mechanism of $\mathrm{Ca}^{2+}$ induced apoptosis.

This study increases the understanding of VDAC gating, metabolite interactions, and transport. However, the exact structural changes induced by metabolites, ions, or voltage remain unknown. Further studies are required to resolve the importance of helix and barrel flexibility for voltage- and ligand-induced gating and to determine alternative conformations. These studies could include solution NMR studies that investigate the helix structure and dynamics under various conditions using RDCs and PREs. The current work shows that RDC values can be measured for hVDAC1 aligned in charged polyacrylamide gels. Quality improvement or application of other alignment strategies (e.g. lanthanide tags) might increase the accuracy. Further experiments could comprise 
investigation of structure, voltage-dependence, and protein interactions of hVDAC1 with a disulfide cross-linked helix, in analogy to the studies performed on bacterial porins. These studies will also provide a more detailed picture about the interplay of helix flexibility and barrel deformation for VDAC interactions and gating.

The extent and localization of dynamics distinguish the mitochondrial porin from bacterial outer membrane proteins. This discrepancy might be related to the requirement of mammalian cells for continuous regulated metabolite exchange and the integration of stress signals into apoptotic pathways. These requirements are considerably different to those of prokaryotic single-cell organisms that naturally lack apoptosis and face changing environments they need to adapt to. In this regard, the highly abundant VDAC channel might be a prototype of outer mitochondrial membrane proteins. Indeed, the structure of VDAC1 is already used as a template for structure predictions of Tom40 (Gessmann et al. 2011), the pore forming component of the translocase of the outer membrane. The knowledge about VDAC dynamics obtained from this study might increase awareness of key dynamic regions and facilitate structural and functional characterization of Tom40 and other mitochondrial outer membrane proteins. It will also be interesting to compare VDAC's features to the dynamics and interactions of other mitochondrial outer membrane proteins in the future. 


\section{References}

Abu-Hamad, S., N. Arbel, D. Calo, L. Arzoine, A. Israelson, N. Keinan, R. BenRomano, O. Friedman, and V. Shoshan-Barmatz. (2009). The VDAC1 Nterminus is essential both for apoptosis and the protective effect of anti-apoptotic proteins. J Cell Sci. 122:1906-16.

Abu-Hamad, S., S. Sivan, and V. Shoshan-Barmatz. (2006). The expression level of the voltage-dependent anion channel controls life and death of the cell. Proc Natl Acad Sci U S A. 103:5787-92.

Abu-Hamad, S., H. Zaid, A. Israelson, E. Nahon, and V. Shoshan-Barmatz. (2008). Hexokinase-I protection against apoptotic cell death is mediated via interaction with the voltage-dependent anion channel-1: mapping the site of binding. $J$ Biol Chem. 283:13482-90.

Accelrys. DelPhi module.

Adams, J.M., and S. Cory. (1998). The Bcl-2 protein family: arbiters of cell survival. Science. 281:1322-6.

Akerboom, T.P., H. Bookelman, P.F. Zuurendonk, R. van der Meer, and J.M. Tager. (1978). Intramitochondrial and extramitochondrial concentrations of adenine nucleotides and inorganic phosphate in isolated hepatocytes from fasted rats. Eur J Biochem. 84:413-20.

Al Bitar, F., N. Roosens, M. Smeyers, M. Vauterin, J. Van Boxtel, M. Jacobs, and F. Homble. (2003). Sequence analysis, transcriptional and posttranscriptional regulation of the rice vdac family. Biochim Biophys Acta. 1625:43-51.

Allen, W.J., J.A. Lemkul, and D.R. Bevan. (2009). GridMAT-MD: a grid-based membrane analysis tool for use with molecular dynamics. J Comput Chem. 30:1952-8.

Angeles, R., J. Devine, K. Barton, M. Smith, and R. McCauley. (1999). Mutation of K234 and K236 in the voltage-dependent anion channel 1 impairs its insertion into the mitochondrial outer membrane. J Bioenerg Biomembr. 31:137-42.

Aram, L., S. Geula, N. Arbel, and V. Shoshan-Barmatz. (2010). VDAC1 cysteine residues: topology and function in channel activity and apoptosis. Biochem $\mathrm{J}$. 427:445-54.

Arbel, N., and V. Shoshan-Barmatz. (2010). Voltage-dependent anion channel 1-based peptides interact with Bcl-2 to prevent antiapoptotic activity. $J$ Biol Chem. 285:6053-62. 
Arbogast, L., A. Majumdar, and J.R. Tolman. (2010). HNCO-based measurement of one-bond amide $15 \mathrm{~N}-1 \mathrm{H}$ couplings with optimized precision. $J$ Biomol NMR. 46:175-89.

Arora, A., F. Abildgaard, J.H. Bushweller, and L.K. Tamm. (2001). Structure of outer membrane protein A transmembrane domain by NMR spectroscopy. Nat Struct Biol. 8:334-8.

Azoulay-Zohar, H., A. Israelson, S. Abu-Hamad, and V. Shoshan-Barmatz. (2004). In self-defence: hexokinase promotes voltage-dependent anion channel closure and prevents mitochondria-mediated apoptotic cell death. Biochem J. 377:347-55.

Bahar, I., A.R. Atilgan, and B. Erman. (1997). Direct evaluation of thermal fluctuations in proteins using a single-parameter harmonic potential. Fold Des. 2:173-81.

Bainbridge, G., H. Mobasheri, G.A. Armstrong, E.J. Lea, and J.H. Lakey. (1998). Voltage-gating of Escherichia coli porin: a cystine-scanning mutagenesis study of loop 3. J Mol Biol. 275:171-6.

Bathori, G., G. Csordas, C. Garcia-Perez, E. Davies, and G. Hajnoczky. (2006). Ca2+dependent control of the permeability properties of the mitochondrial outer membrane and voltage-dependent anion-selective channel (VDAC). J Biol Chem. 281:17347-58.

Bayrhuber, M. (2007). Structural and functional characterisation of the mitochondrial membrane protein human voltage-dependent anion channel (HVDAC) and the membrane protein-targeting Conotoxin Conkunitzin-S1 by solution NMR. In Max Planck Institute for Biophysical Chemistry, Göttingen.

Bayrhuber, M., T. Meins, M. Habeck, S. Becker, K. Giller, S. Villinger, C. Vonrhein, C. Griesinger, M. Zweckstetter, and K. Zeth. (2008). Structure of the human voltage-dependent anion channel. Proc Natl Acad Sci U S A. 105:15370-5.

Benz, R. (1994). Permeation of hydrophilic solutes through mitochondrial outer membranes: review on mitochondrial porins. Biochim Biophys Acta. 1197:16796.

Benz, R., M. Kottke, and D. Brdiczka. (1990). The cationically selective state of the mitochondrial outer membrane pore: a study with intact mitochondria and reconstituted mitochondrial porin. Biochim Biophys Acta. 1022:311-8.

Berezhkovskii, A.M., and S.M. Bezrukov. (2005). Optimizing transport of metabolites through large channels: molecular sieves with and without binding. Biophys $J$. 88:L17-9.

Berger, O., O. Edholm, and F. Jahnig. (1997). Molecular dynamics simulations of a fluid bilayer of dipalmitoylphosphatidylcholine at full hydration, constant pressure, and constant temperature. Biophys J. 72:2002-13.

Bhattacharjya, S., and A. Ramamoorthy. (2009). Multifunctional host defense peptides: functional and mechanistic insights from NMR structures of potent antimicrobial peptides. FEBS J. 276:6465-73.

Bhattacharya, A., M. Revington, and E.R. Zuiderweg. (2010). Measurement and interpretation of $15 \mathrm{~N}-1 \mathrm{H}$ residual dipolar couplings in larger proteins. J Magn Reson. 203:11-28.

Blachly-Dyson, E., and M. Forte. (2001). VDAC channels. IUBMB Life. 52:113-8. 
Blachly-Dyson, E., S. Peng, M. Colombini, and M. Forte. (1990). Selectivity changes in site-directed mutants of the VDAC ion channel: structural implications. Science. 247:1233-6.

Blachly-Dyson, E., E.B. Zambronicz, W.H. Yu, V. Adams, E.R. McCabe, J. Adelman, M. Colombini, and M. Forte. (1993). Cloning and functional expression in yeast of two human isoforms of the outer mitochondrial membrane channel, the voltage-dependent anion channel. J Biol Chem. 268:1835-41.

Bogdanov, M., P. Heacock, Z. Guan, and W. Dowhan. (2010). Plasticity of lipid-protein interactions in the function and topogenesis of the membrane protein lactose permease from Escherichia coli. Proc Natl Acad Sci U S A. 107:15057-15062.

Bolender, N., A. Sickmann, R. Wagner, C. Meisinger, and N. Pfanner. (2008). Multiple pathways for sorting mitochondrial precursor proteins. EMBO Rep. 9:42-9.

Brdiczka, D. (1991). Contact sites between mitochondrial envelope membranes. Structure and function in energy- and protein-transfer. Biochim Biophys Acta. 1071:291-312.

Buchanan, S.K. (1999). Overexpression and refolding of an 80-kDa iron transporter from the outer membrane of Escherichia coli. Biochem Soc Trans. 27:903-8.

Buettner, R., G. Papoutsoglou, E. Scemes, D.C. Spray, and R. Dermietzel. (2000). Evidence for secretory pathway localization of a voltage-dependent anion channel isoform. Proc Natl Acad Sci U S A. 97:3201-6.

Bustamante, E., H.P. Morris, and P.L. Pedersen. (1981). Energy metabolism of tumor cells. Requirement for a form of hexokinase with a propensity for mitochondrial binding. J Biol Chem. 256:8699-704.

Cady, S.D., K. Schmidt-Rohr, J. Wang, C.S. Soto, W.F. Degrado, and M. Hong. (2010). Structure of the amantadine binding site of influenza M2 proton channels in lipid bilayers. Nature. 463:689-92.

Cady, S.D., J. Wang, Y. Wu, W.F. DeGrado, and M. Hong. (2011). Specific binding of adamantane drugs and direction of their polar amines in the pore of the influenza M2 transmembrane domain in lipid bilayers and dodecylphosphocholine micelles determined by NMR spectroscopy. J Am Chem Soc. 133:4274-84.

Casadio, R., I. Jacoboni, A. Messina, and V. De Pinto. (2002). A 3D model of the voltage-dependent anion channel (VDAC). FEBS Lett. 520:1-7.

Cavanagh, J., W.J. Fairbrother, A.G. Palmer III, M. Rance, and N.J. Skelton. (2007). Protein NMR Spectroscopy. Elsevier Academic Press. 885 pp.

Chill, J.H., and F. Naider. (2011). A solution NMR view of protein dynamics in the biological membrane. Curr Opin Struct Biol. 21:627-33.

Choudhary, O.P., R. Ujwal, W. Kowallis, R. Coalson, J. Abramson, and M. Grabe. (2010). The electrostatics of VDAC: implications for selectivity and gating. $J$ Mol Biol. 396:580-92.

Chu, S., A.T. Coey, and G.A. Lorigan. (2010). Solid-state (2)H and (15)N NMR studies of side-chain and backbone dynamics of phospholamban in lipid bilayers: investigation of the N27A mutation. Biochim Biophys Acta. 1798:210-5.

Cierpicki, T., and J.H. Bushweller. (2004). Charged gels as orienting media for measurement of residual dipolar couplings in soluble and integral membrane proteins. J Am Chem Soc. 126:16259-66. 
Cierpicki, T., B. Liang, L.K. Tamm, and J.H. Bushweller. (2006). Increasing the accuracy of solution NMR structures of membrane proteins by application of residual dipolar couplings. High-resolution structure of outer membrane protein A. J Am Chem Soc. 128:6947-51.

Cierpicki, T., and J. Otlewski. (2001). Amide proton temperature coefficients as hydrogen bond indicators in proteins. J Biomol NMR. 21:249-61.

Clore, G.M., and C.D. Schwieters. (2006). Concordance of residual dipolar couplings, backbone order parameters and crystallographic B-factors for a small alpha/beta protein: a unified picture of high probability, fast atomic motions in proteins. $J$ Mol Biol. 355:879-86.

Colombini, M. (1980). Structure and mode of action of a voltage dependent anionselective channel (VDAC) located in the outer mitochondrial membrane. Ann N Y Acad Sci. 341:552-63.

Colombini, M. (1989). Voltage gating in the mitochondrial channel, VDAC. $J M e m b r$ Biol. 111:103-11.

Colombini, M. (2004). VDAC: the channel at the interface between mitochondria and the cytosol. Mol Cell Biochem. 256-257:107-15.

Colombini, M. (2009). The published 3D structure of the VDAC channel: native or not? Trends Biochem Sci. 34:382-9.

Colombini, M., C.L. Yeung, J. Tung, and T. Konig. (1987). The mitochondrial outer membrane channel, VDAC, is regulated by a synthetic polyanion. Biochim Biophys Acta. 905:279-86.

Craik, D.J., and J.A. Wilce. (1997). Studies of protein-ligand interactions by NMR. Methods Mol Biol. 60:195-232.

Crompton, M. (1999). The mitochondrial permeability transition pore and its role in cell death. Biochem J. 341 ( Pt 2):233-49.

Darby, M.K., and I.P. Trayer. (1983). Metal-nucleotide structure at the active sites of the mammalian hexokinases. Eur J Biochem. 129:555-60.

Davis, J.T. (2004). G-quartets 40 years later: from 5'-GMP to molecular biology and supramolecular chemistry. Angew Chem Int Ed Engl. 43:668-98.

de Kroon, A.I., D. Dolis, A. Mayer, R. Lill, and B. de Kruijff. (1997). Phospholipid composition of highly purified mitochondrial outer membranes of rat liver and Neurospora crassa. Is cardiolipin present in the mitochondrial outer membrane? Biochim Biophys Acta. 1325:108-16.

De Pinto, V., J.A. al Jamal, and F. Palmieri. (1993). Location of the dicyclohexylcarbodiimide-reactive glutamate residue in the bovine heart mitochondrial porin. J Biol Chem. 268:12977-82.

De Pinto, V., F. Guarino, A. Guarnera, A. Messina, S. Reina, F.M. Tomasello, V. Palermo, and C. Mazzoni. (2010). Characterization of human VDAC isoforms: a peculiar function for VDAC3? Biochim Biophys Acta. 1797:1268-75.

De Pinto, V., S. Reina, F. Guarino, and A. Messina. (2008). Structure of the voltage dependent anion channel: state of the art. J Bioenerg Biomembr. 40:139-47.

De Pinto, V., F. Tomasello, A. Messina, F. Guarino, R. Benz, D. La Mendola, A. Magri, D. Milardi, and G. Pappalardo. (2007). Determination of the conformation of the 
human VDAC1 N-terminal peptide, a protein moiety essential for the functional properties of the pore. Chembiochem. 8:744-56.

De Pinto, V.D., and F. Palmieri. (1992). Transmembrane arrangement of mitochondrial porin or voltage-dependent anion channel (VDAC). J Bioenerg Biomembr. 24:21-6.

Delaglio, F., S. Grzesiek, G.W. Vuister, G. Zhu, J. Pfeifer, and A. Bax. (1995). NMRPipe: a multidimensional spectral processing system based on UNIX pipes. J Biomol NMR. 6:277-93.

DeLano, W.L. (2003). PyMOL Reference Manual.DeLano Scientific LLC, San Carlos, CA.

Delcour, A.H. (2009). Outer membrane permeability and antibiotic resistance. Biochim Biophys Acta. 1794:808-16.

Dutzler, R., Y.F. Wang, P. Rizkallah, J.P. Rosenbusch, and T. Schirmer. (1996). Crystal structures of various maltooligosaccharides bound to maltoporin reveal a specific sugar translocation pathway. Structure. 4:127-34.

Edrington, T.C., E. Kintz, J.B. Goldberg, and L.K. Tamm. (2011). Structural Basis for the Interaction of Lipopolysaccharide with Outer Membrane Protein H (OprH) from Pseudomonas aeruginosa. J Biol Chem. 286:39211-23.

Eletsky, A., A. Kienhofer, and K. Pervushin. (2001). TROSY NMR with partially deuterated proteins. J Biomol NMR. 20:177-80.

Engelhardt, H., T. Meins, M. Poynor, V. Adams, S. Nussberger, W. Welte, and K. Zeth. (2007). High-level expression, refolding and probing the natural fold of the human voltage-dependent anion channel isoforms I and II. J Membr Biol. 216:93-105.

Englander, S.W., and N.R. Kallenbach. (1983). Hydrogen exchange and structural dynamics of proteins and nucleic acids. Q Rev Biophys. 16:521-655.

Eppens, E.F., N. Saint, P. Van Gelder, R. van Boxtel, and J. Tommassen. (1997). Role of the constriction loop in the gating of outer membrane porin PhoE of Escherichia coli. FEBS Lett. 415:317-20.

Fairman, J.W., N. Noinaj, and S.K. Buchanan. (2011). The structural biology of betabarrel membrane proteins: a summary of recent reports. Curr Opin Struct Biol. 21:523-31.

Feeney, J., J.G. Batchelor, J.P. Albrand, and G.C.K. Roberts. (1979). The effects of intermediate exchange processes on the estimation of equilibrium constants by NMR. Journal of Magnetic Resonance (1969). 33:519-529.

Feenstra, K.A., B. Hess, and H.J.C. Berendsen. (1999). Improving efficiency of large time-scale molecular dynamics simulations of hydrogen-rich systems. Journal of Computational Chemistry. 20:786-798.

Feldmann, G., D. Haouzi, A. Moreau, A.M. Durand-Schneider, A. Bringuier, A. Berson, A. Mansouri, D. Fau, and D. Pessayre. (2000). Opening of the mitochondrial permeability transition pore causes matrix expansion and outer membrane rupture in Fas-mediated hepatic apoptosis in mice. Hepatology. 31:674-83. 
Fernandez, C., C. Hilty, G. Wider, and K. Wuthrich. (2002). Lipid-protein interactions in DHPC micelles containing the integral membrane protein OmpX investigated by NMR spectroscopy. Proc Natl Acad Sci U S A. 99:13533-7.

Florke, H., F.P. Thinnes, H. Winkelbach, U. Stadtmuller, G. Paetzold, C. MorysWortmann, D. Hesse, H. Sternbach, B. Zimmermann, P. Kaufmann-Kolle, and et al. (1994). Channel active mammalian porin, purified from crude membrane fractions of human B lymphocytes and bovine skeletal muscle, reversibly binds adenosine triphosphate (ATP). Biol Chem Hoppe Seyler. 375:513-20.

Forst, D., W. Welte, T. Wacker, and K. Diederichs. (1998). Structure of the sucrosespecific porin ScrY from Salmonella typhimurium and its complex with sucrose. Nat Struct Biol. 5:37-46.

Forte, M., H.R. Guy, and C.A. Mannella. (1987). Molecular genetics of the VDAC ion channel: structural model and sequence analysis. J Bioenerg Biomembr. 19:34150 .

Fushman, D., N. Tjandra, and D. Cowburn. (1998). Direct Measurement of 15N Chemical Shift Anisotropy in Solution. Journal of the American Chemical Society. 120:10947-10952.

Galdiero, S., M. Galdiero, and C. Pedone. (2007). beta-Barrel membrane bacterial proteins: structure, function, assembly and interaction with lipids. Curr Protein Pept Sci. 8:63-82.

Galluzzi, L., O. Kepp, N. Tajeddine, and G. Kroemer. (2008). Disruption of the hexokinase-VDAC complex for tumor therapy. Oncogene. 27:4633-5.

Gautier, A., H.R. Mott, M.J. Bostock, J.P. Kirkpatrick, and D. Nietlispach. (2010). Structure determination of the seven-helix transmembrane receptor sensory rhodopsin II by solution NMR spectroscopy. Nat Struct Mol Biol. 17:768-74.

Gessmann, D., N. Flinner, J. Pfannstiel, A. Schlosinger, E. Schleiff, S. Nussberger, and O. Mirus. (2011). Structural elements of the mitochondrial preproteinconducting channel Tom40 dissolved by bioinformatics and mass spectrometry. Biochim Biophys Acta. 1807:1647-57.

Geula, S., H. Naveed, J. Liang, and V. Shoshan-Barmatz. (2011). Structure-Based Analysis of Vdac1: Defining Oligomer Contact Sites. J Biol Chem.

Gillespie, J.R., and D. Shortle. (1997). Characterization of long-range structure in the denatured state of staphylococcal nuclease. II. Distance restraints from paramagnetic relaxation and calculation of an ensemble of structures. $J \mathrm{Mol}$ Biol. 268:170-84.

Gincel, D., H. Zaid, and V. Shoshan-Barmatz. (2001). Calcium binding and translocation by the voltage-dependent anion channel: a possible regulatory mechanism in mitochondrial function. Biochem J. 358:147-55.

Goddard, T.D., and D.G. Kneller. (2006). SPARKY 3. University of California, San Francisco.

Goncalves, R.P., N. Buzhynskyy, V. Prima, J.N. Sturgis, and S. Scheuring. (2007). Supramolecular assembly of VDAC in native mitochondrial outer membranes. $J$ Mol Biol. 369:413-8. 
Gots, R.E., F.A. Gorin, and S.P. Bessman. (1972). Kinetic enhancement of bound hexokinase activity by mitochondrial respiration. Biochem Biophys Res Commun. 49:1249-55.

Grimm, S., and D. Brdiczka. (2007). The permeability transition pore in cell death. Apoptosis. 12:841-55.

Gromiha, M.M., and M. Suwa. (2007). Current developments on beta-barrel membrane proteins: sequence and structure analysis, discrimination and prediction. Curr Protein Pept Sci. 8:580-99.

Guo, X.W., and C.A. Mannella. (1993). Conformational change in the mitochondrial channel, VDAC, detected by electron cryo-microscopy. Biophys J. 64:545-9.

Guo, X.W., P.R. Smith, B. Cognon, D. D'Arcangelis, E. Dolginova, and C.A. Mannella. (1995). Molecular design of the voltage-dependent, anion-selective channel in the mitochondrial outer membrane. J Struct Biol. 114:41-59.

Hadad, N., A.C. Zable, J.J. Abramson, and V. Shoshan-Barmatz. (1994). Ca2+ binding sites of the ryanodine receptor/Ca2+ release channel of sarcoplasmic reticulum. Low affinity binding site(s) as probed by terbium fluorescence. $J$ Biol Chem. 269:24864-9.

Hansen, S.B., X. Tao, and R. MacKinnon. (2011). Structural basis of PIP2 activation of the classical inward rectifier K+ channel Kir2.2. Nature. 477:495-8.

Hengartner, M.O. (2000). The biochemistry of apoptosis. Nature. 407:770-6.

Hess, B., C. Kutzner, D. van der Spoel, and E. Lindahl. (2008). GROMACS 4: Algorithms for Highly Efficient, Load-Balanced, and Scalable Molecular Simulation. Journal of Chemical Theory and Computation. 4:435-447.

Hill, K., K. Model, M.T. Ryan, K. Dietmeier, F. Martin, R. Wagner, and N. Pfanner. (1998). Tom40 forms the hydrophilic channel of the mitochondrial import pore for preproteins [see comment]. Nature. 395:516-21.

Hiller, S., R.G. Garces, T.J. Malia, V.Y. Orekhov, M. Colombini, and G. Wagner. (2008). Solution structure of the integral human membrane protein VDAC-1 in detergent micelles. Science. 321:1206-10.

Hiller, S., and G. Wagner. (2009). The role of solution NMR in the structure determinations of VDAC-1 and other membrane proteins. Curr Opin Struct Biol. 19:396-401.

Hong, H., G. Szabo, and L.K. Tamm. (2006). Electrostatic couplings in OmpA ionchannel gating suggest a mechanism for pore opening. Nat Chem Biol. 2:627-35.

Hong, M., Y. Zhang, and F. Hu. (2011). Membrane Protein Structure and Dynamics from NMR Spectroscopy. Annu Rev Phys Chem.

Hoogenboom, B.W., K. Suda, A. Engel, and D. Fotiadis. (2007). The supramolecular assemblies of voltage-dependent anion channels in the native membrane. $\mathrm{J} \mathrm{Mol}$ Biol. 370:246-55.

Humphrey, W., A. Dalke, and K. Schulten. (1996). VMD: visual molecular dynamics. $J$ Mol Graph. 14:33-8, 27-8.

Hunte, C., and S. Richers. (2008). Lipids and membrane protein structures. Curr Opin Struct Biol. 18:406-11. 
Hus, J.C., D. Marion, and M. Blackledge. (2000). De novo determination of protein structure by NMR using orientational and long-range order restraints. $J \mathrm{Mol}$ Biol. 298:927-36.

Hwang, P.M., and L.E. Kay. (2005). Solution structure and dynamics of integral membrane proteins by NMR: a case study involving the enzyme PagP. Methods Enzymol. 394:335-50.

Ikegami, T., L. Verdier, P. Sakhaii, S. Grimme, B. Pescatore, K. Saxena, K.M. Fiebig, and C. Griesinger. (2004). Novel techniques for weak alignment of proteins in solution using chemical tags coordinating lanthanide ions. J Biomol NMR. 29:339-49.

Imai, S., M. Osawa, K. Takeuchi, and I. Shimada. (2011). Structural basis underlying the dual gate properties of KcsA. Proc Natl Acad Sci U S A. 107:6216-21.

Inaba, K., S. Murakami, M. Suzuki, A. Nakagawa, E. Yamashita, K. Okada, and K. Ito. (2006). Crystal structure of the DsbB-DsbA complex reveals a mechanism of disulfide bond generation. Cell. 127:789-801.

Israelson, A., S. Abu-Hamad, H. Zaid, E. Nahon, and V. Shoshan-Barmatz. (2007). Localization of the voltage-dependent anion channel-1 Ca2+-binding sites. Cell Calcium. 41:235-44.

Israelson, A., H. Zaid, S. Abu-Hamad, E. Nahon, and V. Shoshan-Barmatz. (2008). Mapping the ruthenium red-binding site of the voltage-dependent anion channel1. Cell Calcium. 43:196-204.

Jorgensen, W.L., J. Chandrasekhar, J.D. Madura, R.W. Impey, and M.L. Klein. (1983). Comparison of simple potential functions for simulating liquid water. The Journal of Chemical Physics. 79:926-935.

Jorgensen, W.L., D.S. Maxwell, and J. Tirado-Rives. (1996). Development and Testing of the OPLS All-Atom Force Field on Conformational Energetics and Properties of Organic Liquids. Journal of the American Chemical Society. 118:1122511236.

Junankar, P.R., A.F. Dulhunty, S.M. Curtis, S.M. Pace, and F.P. Thinnes. (1995). Porintype 1 proteins in sarcoplasmic reticulum and plasmalemma of striated muscle fibres. J Muscle Res Cell Motil. 16:595-610.

Kagan, B.L., and J. Thundimadathil. (2010). Amyloid peptide pores and the beta sheet conformation. Adv Exp Med Biol. 677:150-67.

Kay, L., P. Keifer, and T. Saarinen. (1992). Pure absorption gradient enhanced heteronuclear single quantum correlation spectroscopy with improved sensitivity. Journal of the American Chemical Society. 114:10663-10665.

Keinan, N., D. Tyomkin, and V. Shoshan-Barmatz. (2010). Oligomerization of the mitochondrial protein voltage-dependent anion channel is coupled to the induction of apoptosis. Mol Cell Biol. 30:5698-709.

Kinnally, K.W., H. Tedeschi, and C.A. Mannella. (1987). Evidence for a novel voltageactivated channel in the outer mitochondrial membrane. FEBS Lett. 226:83-7.

Kleckner, I.R., and M.P. Foster. (2011). An introduction to NMR-based approaches for measuring protein dynamics. Biochim Biophys Acta. 1814:942-68. 
Kleinschmidt, J.H., T. den Blaauwen, A.J. Driessen, and L.K. Tamm. (1999). Outer membrane protein A of Escherichia coli inserts and folds into lipid bilayers by a concerted mechanism. Biochemistry. 38:5006-16.

Klingenberg, M. (2008). The ADP and ATP transport in mitochondria and its carrier. Biochim Biophys Acta. 1778:1978-2021.

Kontaxis, G., G.M. Clore, and A. Bax. (2000). Evaluation of cross-correlation effects and measurement of one-bond couplings in proteins with short transverse relaxation times. J Magn Reson. 143:184-96.

Koppel, D.A., K.W. Kinnally, P. Masters, M. Forte, E. Blachly-Dyson, and C.A. Mannella. (1998). Bacterial expression and characterization of the mitochondrial outer membrane channel. Effects of n-terminal modifications. $J$ Biol Chem. 273:13794-800.

Krishna, M.M., L. Hoang, Y. Lin, and S.W. Englander. (2004). Hydrogen exchange methods to study protein folding. Methods. 34:51-64.

Langosch, D., and I.T. Arkin. (2009). Interaction and conformational dynamics of membrane-spanning protein helices. Protein Sci. 18:1343-58.

Lee, A.C., M. Zizi, and M. Colombini. (1994). Beta-NADH decreases the permeability of the mitochondrial outer membrane to ADP by a factor of $6 . J$ Biol Chem. 269:30974-80.

Lee, D., C. Hilty, G. Wider, and K. Wuthrich. (2006). Effective rotational correlation times of proteins from NMR relaxation interference. J Magn Reson. 178:72-6.

Lee, D., K.F. Walter, A.K. Bruckner, C. Hilty, S. Becker, and C. Griesinger. (2008). Bilayer in small bicelles revealed by lipid-protein interactions using NMR spectroscopy. J Am Chem Soc. 130:13822-3.

Lemasters, J.J., and E. Holmuhamedov. (2006). Voltage-dependent anion channel (VDAC) as mitochondrial governator--thinking outside the box. Biochim Biophys Acta. 1762:181-90.

Liang, B., A. Arora, and L.K. Tamm. (2010). Fast-time scale dynamics of outer membrane protein A by extended model-free analysis of NMR relaxation data. Biochim Biophys Acta. 1798:68-76.

Liang, B., and L.K. Tamm. (2007). Structure of outer membrane protein G by solution NMR spectroscopy. Proc Natl Acad Sci U S A. 104:16140-5.

Lipsitz, R.S., and N. Tjandra. (2004). Residual dipolar couplings in NMR structure analysis. Annu Rev Biophys Biomol Struct. 33:387-413.

Liu, N., and A.H. Delcour. (1998). The spontaneous gating activity of OmpC porin is affected by mutations of a putative hydrogen bond network or of a salt bridge between the L3 loop and the barrel. Protein Eng. 11:797-802.

Liu, X., C.N. Kim, J. Yang, R. Jemmerson, and X. Wang. (1996). Induction of apoptotic program in cell-free extracts: requirement for dATP and cytochrome c. Cell. $86: 147-57$.

Loria, J.P., M. Rance, and A.G. Palmer. (1999a). A Relaxation-Compensated CarrPurcell-Meiboom-Gill Sequence for Characterizing Chemical Exchange by NMR Spectroscopy. Journal of the American Chemical Society. 121:2331-2332.

Loria, J.P., M. Rance, and A.G. Palmer, 3rd. (1999b). A TROSY CPMG sequence for characterizing chemical exchange in large proteins. J Biomol NMR. 15:151-5. 
Lorieau, J., L. Yao, and A. Bax. (2008). Liquid crystalline phase of G-tetrad DNA for NMR study of detergent-solubilized proteins. J Am Chem Soc. 130:7536-7.

Lou, H., M. Chen, S.S. Black, S.R. Bushell, M. Ceccarelli, T. Mach, K. Beis, A.S. Low, V.A. Bamford, I.R. Booth, H. Bayley, and J.H. Naismith. (2011). Altered Antibiotic Transport in OmpC Mutants Isolated from a Series of Clinical Strains of Multi-Drug Resistant E. coli. PLoS One. 6:e25825.

Malia, T.J., and Massachusetts Institute of Technology. Dept. of Chemistry. (2006). NMR structural and functional studies of the mithochondrial outer membrane protein VDAC / by Thomas J. Malia. 195 leaves.

Malia, T.J., and G. Wagner. (2007). NMR structural investigation of the mitochondrial outer membrane protein VDAC and its interaction with antiapoptotic Bcl-xL. Biochemistry. 46:514-25.

Mannella, C.A. (1982). Structure of the outer mitochondrial membrane: ordered arrays of porelike subunits in outer-membrane fractions from Neurospora crassa mitochondria. J Cell Biol. 94:680-7.

Mannella, C.A. (1997). Minireview: on the structure and gating mechanism of the mitochondrial channel, VDAC. J Bioenerg Biomembr. 29:525-31.

Mannella, C.A. (1998). Conformational changes in the mitochondrial channel protein, VDAC, and their functional implications. J Struct Biol. 121:207-18.

Martin, R.B., and F.S. Richardson. (1979). Lanthanides as probes for calcium in biological systems. $Q$ Rev Biophys. 12:181-209.

McCabe, E.R. (1994). Microcompartmentation of energy metabolism at the outer mitochondrial membrane: role in diabetes mellitus and other diseases. $J$ Bioenerg Biomembr. 26:317-25.

Meins, T. (2007). In Max Planck Institute for Biochemistry, Munich.

Meisinger, C., S. Pfannschmidt, M. Rissler, D. Milenkovic, T. Becker, D. Stojanovski, M.J. Youngman, R.E. Jensen, A. Chacinska, B. Guiard, N. Pfanner, and N. Wiedemann. (2007). The morphology proteins Mdm12/Mmm1 function in the major beta-barrel assembly pathway of mitochondria. Embo J. 26:2229-39.

Meisinger, C., M. Rissler, A. Chacinska, L.K. Szklarz, D. Milenkovic, V. Kozjak, B. Schonfisch, C. Lohaus, H.E. Meyer, M.P. Yaffe, B. Guiard, N. Wiedemann, and N. Pfanner. (2004). The mitochondrial morphology protein Mdm10 functions in assembly of the preprotein translocase of the outer membrane. Dev Cell. 7:6171.

Meyer, J.E., M. Hofnung, and G.E. Schulz. (1997). Structure of maltoporin from Salmonella typhimurium ligated with a nitrophenyl-maltotrioside. J Mol Biol. 266:761-75.

Meyer, J.E., and G.E. Schulz. (1997). Energy profile of maltooligosaccharide permeation through maltoporin as derived from the structure and from a statistical analysis of saccharide-protein interactions. Protein Sci. 6:1084-91.

Moraes, T.F., M. Bains, R.E. Hancock, and N.C. Strynadka. (2007). An arginine ladder in OprP mediates phosphate-specific transfer across the outer membrane. Nat Struct Mol Biol. 14:85-7. 
Moran, O., M. Sciancalepore, G. Sandri, E. Panfili, R. Bassi, C. Ballarin, and M.C. Sorgato. (1992). Ionic permeability of the mitochondrial outer membrane. Eur Biophys J. 20:311-9.

Mueller, P., D.O. Rudin, H.T. Tien, and W.C. Wescott. (1962). Reconstitution of cell membrane structure in vitro and its transformation into an excitable system. Nature. 194:979-80.

Nakashima, R.A., P.S. Mangan, M. Colombini, and P.L. Pedersen. (1986). Hexokinase receptor complex in hepatoma mitochondria: evidence from N,N'dicyclohexylcarbodiimide-labeling studies for the involvement of the poreforming protein VDAC. Biochemistry. 25:1015-21.

Narita, M., S. Shimizu, T. Ito, T. Chittenden, R.J. Lutz, H. Matsuda, and Y. Tsujimoto. (1998). Bax interacts with the permeability transition pore to induce permeability transition and cytochrome $\mathrm{c}$ release in isolated mitochondria. Proc Natl Acad Sci U S A. 95:14681-6.

Naveed, H., R. Jackups, Jr., and J. Liang. (2009). Predicting weakly stable regions, oligomerization state, and protein-protein interfaces in transmembrane domains of outer membrane proteins. Proc Natl Acad Sci U S A. 106:12735-40.

Nestorovich, E.M., C. Danelon, M. Winterhalter, and S.M. Bezrukov. (2002). Designed to penetrate: time-resolved interaction of single antibiotic molecules with bacterial pores. Proc Natl Acad Sci U S A. 99:9789-94.

Nietlispach, D., and A. Gautier. (2011). Solution NMR studies of polytopic alphahelical membrane proteins. Curr Opin Struct Biol. 21:497-508.

Nyholm, T.K., S. Ozdirekcan, and J.A. Killian. (2007). How protein transmembrane segments sense the lipid environment. Biochemistry. 46:1457-65.

Palmer III, A.G., J. Cavanagh, P.E. Wright, and M. Rance. (1991). Sensitivity improvement in proton-detected two-dimensional heteronuclear correlation NMR spectroscopy. Journal of Magnetic Resonance (1969). 93:151-170.

Park, S.H., W.S. Son, R. Mukhopadhyay, H. Valafar, and S.J. Opella. (2009). Phageinduced alignment of membrane proteins enables the measurement and structural analysis of residual dipolar couplings with dipolar waves and lambdamaps. J Am Chem Soc. 131:14140-1.

Parker, M.J., M. Aulton-Jones, A.M. Hounslow, and C.J. Craven. (2004). A combinatorial selective labeling method for the assignment of backbone amide NMR resonances. $J$ Am Chem Soc. 126:5020-1.

Pastorino, J.G., N. Shulga, and J.B. Hoek. (2002). Mitochondrial binding of hexokinase II inhibits Bax-induced cytochrome c release and apoptosis. J Biol Chem. 277:7610-8.

Pautsch, A., and G.E. Schulz. (1998). Structure of the outer membrane protein A transmembrane domain. Nat Struct Biol. 5:1013-7.

Peng, S., E. Blachly-Dyson, M. Forte, and M. Colombini. (1992). Large scale rearrangement of protein domains is associated with voltage gating of the VDAC channel. Biophys J. 62:123-31; discussion 131-5.

Perevoshchikova, I.V., S.D. Zorov, E.A. Kotova, D.B. Zorov, and Y.N. Antonenko. (2010). Hexokinase inhibits flux of fluorescently labeled ATP through mitochondrial outer membrane porin. FEBS Lett. 584:2397-402. 
Pervushin, K., R. Riek, G. Wider, and K. Wuthrich. (1997). Attenuated T2 relaxation by mutual cancellation of dipole-dipole coupling and chemical shift anisotropy indicates an avenue to NMR structures of very large biological macromolecules in solution. Proc Natl Acad Sci U S A. 94:12366-71.

Pfaff, E., and M. Klingenberg. (1968). Adenine nucleotide translocation of mitochondria. 1. Specificity and control. Eur J Biochem. 6:66-79.

Phale, P.S., T. Schirmer, A. Prilipov, K.L. Lou, A. Hardmeyer, and J.P. Rosenbusch. (1997). Voltage gating of Escherichia coli porin channels: role of the constriction loop. Proc Natl Acad Sci U S A. 94:6741-5.

Pongprayoon, P., O. Beckstein, C.L. Wee, and M.S. Sansom. (2009). Simulations of anion transport through OprP reveal the molecular basis for high affinity and selectivity for phosphate. Proc Natl Acad Sci U S A. 106:21614-8.

Popp, B., D.A. Court, R. Benz, W. Neupert, and R. Lill. (1996). The role of the N and C termini of recombinant Neurospora mitochondrial porin in channel formation and voltage-dependent gating. J Biol Chem. 271:13593-9.

Rahmani, Z., C. Maunoury, and A. Siddiqui. (1998). Isolation of a novel human voltage-dependent anion channel gene. Eur J Hum Genet. 6:337-40.

Rapizzi, E., P. Pinton, G. Szabadkai, M.R. Wieckowski, G. Vandecasteele, G. Baird, R.A. Tuft, K.E. Fogarty, and R. Rizzuto. (2002). Recombinant expression of the voltage-dependent anion channel enhances the transfer of $\mathrm{Ca} 2+$ microdomains to mitochondria. J Cell Biol. 159:613-24.

Ravagnan, L., T. Roumier, and G. Kroemer. (2002). Mitochondria, the killer organelles and their weapons. J Cell Physiol. 192:131-7.

Reed, K.C., and F.L. Bygrave. (1974). The inhibition of mitochondrial calcium transport by lanthanides and ruthenium red. Biochem J. 140:143-55.

Renault, M., O. Saurel, J. Czaplicki, P. Demange, V. Gervais, F. Lohr, V. Reat, M. Piotto, and A. Milon. (2009). Solution state NMR structure and dynamics of KpOmpA, a 210 residue transmembrane domain possessing a high potential for immunological applications. J Mol Biol. 385:117-30.

Ringe, D., and G.A. Petsko. (1986). Study of protein dynamics by X-ray diffraction. Methods Enzymol. 131:389-433.

Rostovtseva, T., and M. Colombini. (1997). VDAC channels mediate and gate the flow of ATP: implications for the regulation of mitochondrial function. Biophys $J$. 72:1954-62.

Rostovtseva, T.K., B. Antonsson, M. Suzuki, R.J. Youle, M. Colombini, and S.M. Bezrukov. (2004). Bid, but not Bax, regulates VDAC channels. J Biol Chem. 279:13575-83.

Rostovtseva, T.K., and S.M. Bezrukov. (1998). ATP transport through a single mitochondrial channel, VDAC, studied by current fluctuation analysis. Biophys J. 74:2365-73.

Rostovtseva, T.K., and S.M. Bezrukov. (2008). VDAC regulation: role of cytosolic proteins and mitochondrial lipids. J Bioenerg Biomembr. 40:163-70.

Rostovtseva, T.K., A. Komarov, S.M. Bezrukov, and M. Colombini. (2002). Dynamics of nucleotides in VDAC channels: structure-specific noise generation. Biophys J. 82:193-205. 
Rostovtseva, T.K., W. Tan, and M. Colombini. (2005). On the role of VDAC in apoptosis: fact and fiction. J Bioenerg Biomembr. 37:129-42.

Rui, H., K.I. Lee, R.W. Pastor, and W. Im. (2011). Molecular dynamics studies of ion permeation in VDAC. Biophys J. 100:602-10.

Runke, G., E. Maier, J.D. O'Neil, R. Benz, and D.A. Court. (2000). Functional characterization of the conserved "GLK" motif in mitochondrial porin from Neurospora crassa. J Bioenerg Biomembr. 32:563-70.

Russell, R.B., and D.S. Eggleston. (2000). New roles for structure in biology and drug discovery. Nat Struct Biol. 7 Suppl:928-30.

Salnikov, E., C. Aisenbrey, V. Vidovic, and B. Bechinger. (2010). Solid-state NMR approaches to measure topological equilibria and dynamics of membrane polypeptides. Biochim Biophys Acta. 1798:258-65.

Salzmann, M., K. Pervushin, G. Wider, H. Senn, and K. Wuthrich. (1998). TROSY in triple-resonance experiments: new perspectives for sequential NMR assignment of large proteins. Proc Natl Acad Sci U S A. 95:13585-90.

Sampson, M.J., R.S. Lovell, and W.J. Craigen. (1997). The murine voltage-dependent anion channel gene family. Conserved structure and function. J Biol Chem. 272:18966-73.

Saraste, M. (1999). Oxidative phosphorylation at the fin de siecle. Science. 283:148893.

Saxena, K., V. Drosou, E. Maier, R. Benz, and B. Ludwig. (1999). Ion selectivity reversal and induction of voltage-gating by site-directed mutations in the Paracoccus denitrificans porin. Biochemistry. 38:2206-12.

Schein, S.J., M. Colombini, and A. Finkelstein. (1976). Reconstitution in planar lipid bilayers of a voltage-dependent anion-selective channel obtained from paramecium mitochondria. J Membr Biol. 30:99-120.

Schirmer, T., T.A. Keller, Y.F. Wang, and J.P. Rosenbusch. (1995). Structural basis for sugar translocation through maltoporin channels at 3.1 A resolution. Science. 267:512-4.

Schleucher, J., M. Schwendinger, M. Sattler, P. Schmidt, O. Schedletzky, S.J. Glaser, O.W. Sorensen, and C. Griesinger. (1994). A general enhancement scheme in heteronuclear multidimensional NMR employing pulsed field gradients. $J$ Biomol NMR. 4:301-6.

Schmid, B., L. Maveyraud, M. Kromer, and G.E. Schulz. (1998). Porin mutants with new channel properties. Protein Sci. 7:1603-11.

Schneider, R., M. Etzkorn, K. Giller, V. Daebel, J. Eisfeld, M. Zweckstetter, C. Griesinger, S. Becker, and A. Lange. (2010). The native conformation of the human VDAC1 N-terminus. Angew Chem Int Ed Engl. 49:1882-1885.

Schoenmakers, T.J., G.J. Visser, G. Flik, and A.P. Theuvenet. (1992). CHELATOR: an improved method for computing metal ion concentrations in physiological solutions. Biotechniques. 12:870-4, 876-9.

Schulz, G.E. (2000). beta-Barrel membrane proteins. Curr Opin Struct Biol. 10:443-7.

Schulz, G.E. (2002). The structure of bacterial outer membrane proteins. Biochim Biophys Acta. 1565:308-17. 
Schwarzinger, S., G.J. Kroon, T.R. Foss, J. Chung, P.E. Wright, and H.J. Dyson. (2001). Sequence-dependent correction of random coil NMR chemical shifts. $J$ Am Chem Soc. 123:2970-8.

Sevvana, M., V. Vijayan, M. Zweckstetter, S. Reinelt, D.R. Madden, R. Herbst-Irmer, G.M. Sheldrick, M. Bott, C. Griesinger, and S. Becker. (2008). A ligand-induced switch in the periplasmic domain of sensor histidine kinase CitA. J Mol Biol. 377:512-23.

Shafir, I., W. Feng, and V. Shoshan-Barmatz. (1998). Dicyclohexylcarbodiimide interaction with the voltage-dependent anion channel from sarcoplasmic reticulum. Eur J Biochem. 253:627-36.

Shi, Y., J. Chen, C. Weng, R. Chen, Y. Zheng, Q. Chen, and H. Tang. (2003). Identification of the protein-protein contact site and interaction mode of human VDAC1 with Bcl-2 family proteins. Biochem Biophys Res Commun. 305:98996.

Shimizu, S., T. Ide, T. Yanagida, and Y. Tsujimoto. (2000). Electrophysiological study of a novel large pore formed by Bax and the voltage-dependent anion channel that is permeable to cytochrome c. J Biol Chem. 275:12321-5.

Shimizu, S., Y. Matsuoka, Y. Shinohara, Y. Yoneda, and Y. Tsujimoto. (2001). Essential role of voltage-dependent anion channel in various forms of apoptosis in mammalian cells. J Cell Biol. 152:237-50.

Shoshan-Barmatz, V., V. De Pinto, M. Zweckstetter, Z. Raviv, N. Keinan, and N. Arbel. (2010a). VDAC, a multi-functional mitochondrial protein regulating cell life and death. Mol Aspects Med. 31:227-285.

Shoshan-Barmatz, V., A. Israelson, D. Brdiczka, and S.S. Sheu. (2006). The voltagedependent anion channel (VDAC): function in intracellular signalling, cell life and cell death. Curr Pharm Des. 12:2249-70.

Shoshan-Barmatz, V., N. Keinan, S. Abu-Hamad, D. Tyomkin, and L. Aram. (2010b). Apoptosis is regulated by the VDAC1 N-terminal region and by VDAC oligomerization: release of cytochrome c, AIF and Smac/Diablo. Biochim Biophys Acta.

Shoshan-Barmatz, V., N. Keinan, and H. Zaid. (2008). Uncovering the role of VDAC in the regulation of cell life and death. J Bioenerg Biomembr. 40:183-91.

Sickmeier, M., J.A. Hamilton, T. LeGall, V. Vacic, M.S. Cortese, A. Tantos, B. Szabo, P. Tompa, J. Chen, V.N. Uversky, Z. Obradovic, and A.K. Dunker. (2007). DisProt: the Database of Disordered Proteins. Nucleic Acids Res. 35:D786-93.

Simamura, E., H. Shimada, T. Hatta, and K. Hirai. (2008). Mitochondrial voltagedependent anion channels (VDACs) as novel pharmacological targets for anticancer agents. J Bioenerg Biomembr. 40:213-7.

Sklenáŕ, V., and A. Bax. (1987). Spin-echo water suppression for the generation of pure-phase two-dimensional NMR spectra. Journal of Magnetic Resonance (1969). 74:469-479.

Smith, M.D., M. Petrak, P.D. Boucher, K.N. Barton, L. Carter, G. Reddy, E. BlachlyDyson, M. Forte, J. Price, K. Verner, and et al. (1995). Lysine residues at positions 234 and 236 in yeast porin are involved in its assembly into the mitochondrial outer membrane. J Biol Chem. 270:28331-6. 
Smith, R.M., and R.A. Alberty. (1956a). The apparent stability constants of ionic complexes of various adenosine phosphates and divalent cations. $J$ Am Chem Soc. 78:2376-80.

Smith, R.M., and R.A. Alberty. (1956b). The Apparent Stability Constants of Ionic Complexes of Various Adenosine Phosphates with Monovalent Cations. The Journal of Physical Chemistry. 60:180-184.

Song, J., C. Midson, E. Blachly-Dyson, M. Forte, and M. Colombini. (1998a). The sensor regions of VDAC are translocated from within the membrane to the surface during the gating processes. Biophys J. 74:2926-44.

Song, J., C. Midson, E. Blachly-Dyson, M. Forte, and M. Colombini. (1998b). The topology of VDAC as probed by biotin modification. J Biol Chem. 273:2440613.

Sukhan, A., and R.E. Hancock. (1996). The role of specific lysine residues in the passage of anions through the Pseudomonas aeruginosa porin OprP. $J$ Biol Chem. 271:21239-42.

Susin, S.A., N. Zamzami, M. Castedo, T. Hirsch, P. Marchetti, A. Macho, E. Daugas, M. Geuskens, and G. Kroemer. (1996). Bcl-2 inhibits the mitochondrial release of an apoptogenic protease. $J$ Exp Med. 184:1331-41.

Szabo, I., V. De Pinto, and M. Zoratti. (1993). The mitochondrial permeability transition pore may comprise VDAC molecules. II. The electrophysiological properties of VDAC are compatible with those of the mitochondrial megachannel. FEBS Lett. 330:206-10.

Tait, S.W., and D.R. Green. (2010). Mitochondria and cell death: outer membrane permeabilization and beyond. Nat Rev Mol Cell Biol. 11:621-32.

Tajeddine, N., L. Galluzzi, O. Kepp, E. Hangen, E. Morselli, L. Senovilla, N. Araujo, G. Pinna, N. Larochette, N. Zamzami, N. Modjtahedi, A. Harel-Bellan, and G. Kroemer. (2008). Hierarchical involvement of Bak, VDAC1 and Bax in cisplatin-induced cell death. Oncogene. 27:4221-32.

Tamm, L.K., H. Hong, and B. Liang. (2004). Folding and assembly of beta-barrel membrane proteins. Biochim Biophys Acta. 1666:250-63.

Tan, W., and M. Colombini. (2007). VDAC closure increases calcium ion flux. Biochim Biophys Acta. 1768:2510-5.

Thomas, L., E. Blachly-Dyson, M. Colombini, and M. Forte. (1993). Mapping of residues forming the voltage sensor of the voltage-dependent anion-selective channel. Proc Natl Acad Sci U S A. 90:5446-9.

Tirion, M.M. (1996). Large Amplitude Elastic Motions in Proteins from a SingleParameter, Atomic Analysis. Phys Rev Lett. 77:1905-1908.

Tischler, M.E., D. Friedrichs, K. Coll, and J.R. Williamson. (1977). Pyridine nucleotide distributions and enzyme mass action ratios in hepatocytes from fed and starved rats. Arch Biochem Biophys. 184:222-36.

Tjandra, N., and A. Bax. (1997). Direct measurement of distances and angles in biomolecules by NMR in a dilute liquid crystalline medium. Science. 278:11114. 
Tolman, J.R., J.M. Flanagan, M.A. Kennedy, and J.H. Prestegard. (1995). Nuclear magnetic dipole interactions in field-oriented proteins: information for structure determination in solution. Proc Natl Acad Sci U S A. 92:9279-83.

Torbet, J., and G. Maret. (1981). High-field magnetic birefringence study of the structure of rodlike phages Pf1 and fd in solution. Biopolymers. 20:2657-69.

Traaseth, N.J., and G. Veglia. (2010). Probing excited states and activation energy for the integral membrane protein phospholamban by NMR CPMG relaxation dispersion experiments. Biochim Biophys Acta. 1798:77-81.

Traut, T.W. (1994). Physiological concentrations of purines and pyrimidines. Mol Cell Biochem. 140:1-22.

Tsujimoto, Y., and S. Shimizu. (2000). Bcl-2 family: life-or-death switch. FEBS Lett. 466:6-10.

Tugarinov, V., and L.E. Kay. (2003). Ile, Leu, and Val methyl assignments of the 723residue malate synthase $G$ using a new labeling strategy and novel NMR methods. J Am Chem Soc. 125:13868-78.

Ujwal, R., D. Cascio, V. Chaptal, P. Ping, and J. Abramson. (2009). Crystal packing analysis of murine VDAC1 crystals in a lipidic environment reveals novel insights on oligomerization and orientation. Channels (Austin). 3:167-70.

Ujwal, R., D. Cascio, J.P. Colletier, S. Faham, J. Zhang, L. Toro, P. Ping, and J. Abramson. (2008). The crystal structure of mouse VDAC1 at 2.3 A resolution reveals mechanistic insights into metabolite gating. Proc Natl Acad Sci U S A. 105:17742-7.

Valiyaveetil, F., J. Hermolin, and R.H. Fillingame. (2002). pH dependent inactivation of solubilized F1F0 ATP synthase by dicyclohexylcarbodiimide: $\mathrm{pK}(\mathrm{a})$ of detergent unmasked aspartyl-61 in Escherichia coli subunit c. Biochim Biophys Acta. 1553:296-301.

Van Horn, W.D., H.J. Kim, C.D. Ellis, A. Hadziselimovic, E.S. Sulistijo, M.D. Karra, C. Tian, F.D. Sonnichsen, and C.R. Sanders. (2009). Solution nuclear magnetic resonance structure of membrane-integral diacylglycerol kinase. Science. 324:1726-9.

Vander Heiden, M.G., N.S. Chandel, P.T. Schumacker, and C.B. Thompson. (1999). Bcl-xL prevents cell death following growth factor withdrawal by facilitating mitochondrial ATP/ADP exchange. Mol Cell. 3:159-67.

Vander Heiden, M.G., X.X. Li, E. Gottleib, R.B. Hill, C.B. Thompson, and M. Colombini. (2001). Bcl-xL promotes the open configuration of the voltagedependent anion channel and metabolite passage through the outer mitochondrial membrane. J Biol Chem. 276:19414-9.

Veglia, G., and A. Ramamoorthy. (2010). Special issue on "membrane protein dynamics: correlating structure to function". Biochim Biophys Acta. 1798:65-7.

Villinger, S., R. Briones, K. Giller, U. Zachariae, A. Lange, B.L. de Groot, C. Griesinger, S. Becker, and M. Zweckstetter. (2010). Functional dynamics in the voltage-dependent anion channel. Proc Natl Acad Sci U S A. 107:22546-51.

Vriend, G. (1990). WHAT IF: a molecular modeling and drug design program. $J$ Mol Graph. 8:52-6, 29. 
Wagner, D.S., L.G. Melton, Y. Yan, B.W. Erickson, and R.J. Anderegg. (1994). Deuterium exchange of alpha-helices and beta-sheets as monitored by electrospray ionization mass spectrometry. Protein Sci. 3:1305-14.

Walker, J.E., M. Saraste, M.J. Runswick, and N.J. Gay. (1982). Distantly related sequences in the alpha- and beta-subunits of ATP synthase, myosin, kinases and other ATP-requiring enzymes and a common nucleotide binding fold. Embo J. 1:945-51.

Wang, C., M. Rance, and A.G. Palmer, 3rd. (2003). Mapping chemical exchange in proteins with MW > 50 kD. J Am Chem Soc. 125:8968-9.

Wang, G. (2008). NMR of membrane-associated peptides and proteins. Curr Protein Pept Sci. 9:50-69.

Wang, J., R.M. Wolf, J.W. Caldwell, P.A. Kollman, and D.A. Case. (2004). Development and testing of a general amber force field. J Comput Chem. 25:1157-74.

Weiss, M.S., and G.E. Schulz. (1992). Structure of porin refined at 1.8 A resolution. $J$ Mol Biol. 227:493-509.

Wiedemann, N., V. Kozjak, A. Chacinska, B. Schonfisch, S. Rospert, M.T. Ryan, N. Pfanner, and C. Meisinger. (2003). Machinery for protein sorting and assembly in the mitochondrial outer membrane. Nature. 424:565-71.

Wishart, D.S., C.G. Bigam, A. Holm, R.S. Hodges, and B.D. Sykes. (1995). 1H, 13C and $15 \mathrm{~N}$ random coil NMR chemical shifts of the common amino acids. I. Investigations of nearest-neighbor effects. J Biomol NMR. 5:67-81.

Wolf, M.G., M. Hoefling, C. Aponte-Santamaria, H. Grubmuller, and G. Groenhof. (2010). g_membed: Efficient insertion of a membrane protein into an equilibrated lipid bilayer with minimal perturbation. J Comput Chem. 31:216974.

Wunder, U.R., and M. Colombini. (1991). Patch clamping VDAC in liposomes containing whole mitochondrial membranes. J Membr Biol. 123:83-91.

Xia, Y., K. Sze, and G. Zhu. (2000). Transverse relaxation optimized 3D and 4D $15 \mathrm{n} / 15 \mathrm{~N}$ separated NOESY experiments of $15 \mathrm{~N}$ labeled proteins. $J$ Biomol NMR. 18:261-8.

Xu, X., W. Decker, M.J. Sampson, W.J. Craigen, and M. Colombini. (1999). Mouse VDAC isoforms expressed in yeast: channel properties and their roles in mitochondrial outer membrane permeability. J Membr Biol. 170:89-102.

Yang, D., R.A. Venters, G.A. Mueller, W.Y. Choy, and L.E. Kay. (1999). TROSYbased $\mathrm{HNCO}$ pulse sequences for the measurement of $1 \mathrm{HN}-15 \mathrm{~N}, 15 \mathrm{~N}-13 \mathrm{CO}$, $1 \mathrm{HN}-13 \mathrm{CO}, 13 \mathrm{CO}-13 \mathrm{C} \alpha$ and $1 \mathrm{HN}-13 \mathrm{C} \alpha$ dipolar couplings in $15 \mathrm{~N}, 13 \mathrm{C}, 2 \mathrm{H}-$ labeled proteins. Journal of Biomolecular NMR. 14:333-343.

Yang, J., M.L. Tasayco, and T. Polenova. (2009). Dynamics of reassembled thioredoxin studied by magic angle spinning NMR: snapshots from different time scales. $J$ Am Chem Soc. 131:13690-702.

Yang, L.W., A.J. Rader, X. Liu, C.J. Jursa, S.C. Chen, H.A. Karimi, and I. Bahar. (2006). oGNM: online computation of structural dynamics using the Gaussian Network Model. Nucleic Acids Res. 34:W24-31. 
Yehezkel, G., S. Abu-Hamad, and V. Shoshan-Barmatz. (2007). An N-terminal nucleotide-binding site in VDAC1: involvement in regulating mitochondrial function. J Cell Physiol. 212:551-61.

Yehezkel, G., N. Hadad, H. Zaid, S. Sivan, and V. Shoshan-Barmatz. (2006). Nucleotide-binding sites in the voltage-dependent anion channel: characterization and localization. J Biol Chem. 281:5938-46.

Yildiz, O., K.R. Vinothkumar, P. Goswami, and W. Kuhlbrandt. (2006). Structure of the monomeric outer-membrane porin OmpG in the open and closed conformation. EMBO J. 25:3702-13.

Ying, J., J.H. Chill, J.M. Louis, and A. Bax. (2007). Mixed-time parallel evolution in multiple quantum NMR experiments: sensitivity and resolution enhancement in heteronuclear NMR. J Biomol NMR. 37:195-204.

Zachariae, U., T. Kluhspies, S. De, H. Engelhardt, and K. Zeth. (2006). High resolution crystal structures and molecular dynamics studies reveal substrate binding in the porin Omp32. J Biol Chem. 281:7413-20.

Zachariae, U., R. Schneider, P. Velisetty, A. Lange, D. Seeliger, S.J. Wacker, Y. Karimi-Nejad, G. Vriend, S. Becker, O. Pongs, M. Baldus, and B.L. de Groot. (2008). The molecular mechanism of toxin-induced conformational changes in a potassium channel: relation to C-type inactivation. Structure. 16:747-54.

Zaid, H., S. Abu-Hamad, A. Israelson, I. Nathan, and V. Shoshan-Barmatz. (2005). The voltage-dependent anion channel-1 modulates apoptotic cell death. Cell Death Differ. 12:751-60.

Zalk, R., A. Israelson, E.S. Garty, H. Azoulay-Zohar, and V. Shoshan-Barmatz. (2005). Oligomeric states of the voltage-dependent anion channel and cytochrome c release from mitochondria. Biochem J. 386:73-83.

Zeth, K., and M. Thein. (2010). Porins in prokaryotes and eukaryotes: common themes and variations. Biochem J. 431:13-22.

Zheng, Y., Y. Shi, C. Tian, C. Jiang, H. Jin, J. Chen, A. Almasan, H. Tang, and Q. Chen. (2004). Essential role of the voltage-dependent anion channel (VDAC) in mitochondrial permeability transition pore opening and cytochrome c release induced by arsenic trioxide. Oncogene. 23:1239-47.

Zhou, Y., T. Cierpicki, R.H. Jimenez, S.M. Lukasik, J.F. Ellena, D.S. Cafiso, H. Kadokura, J. Beckwith, and J.H. Bushweller. (2008). NMR solution structure of the integral membrane enzyme DsbB: functional insights into DsbB-catalyzed disulfide bond formation. Mol Cell. 31:896-908.

Zhu, G., X.M. Kong, and K.H. Sze. (1999). Gradient and sensitivity enhancement of 2D TROSY with water flip-back, 3D NOESY-TROSY and TOCSY-TROSY experiments. Journal of Biomolecular NMR. 13:77-81.

Zhu, G., Y. Xia, L.K. Nicholson, and K.H. Sze. (2000). Protein dynamics measurements by TROSY-based NMR experiments. J Magn Reson. 143:423-6.

Zimmerberg, J., and V.A. Parsegian. (1986). Polymer inaccessible volume changes during opening and closing of a voltage-dependent ionic channel. Nature. 323:36-9. 
Zizi, M., M. Forte, E. Blachly-Dyson, and M. Colombini. (1994). NADH regulates the gating of VDAC, the mitochondrial outer membrane channel. J Biol Chem. 269:1614-6.

Zoratti, M., and I. Szabo. (1995). The mitochondrial permeability transition. Biochim Biophys Acta. 1241:139-76.

Zou, H., M. Zheng, X. Luo, W. Zhu, K. Chen, J. Shen, and H. Jiang. (2008). Dynamic mechanism of fatty acid transport across cellular membranes through FadL: molecular dynamics simulations. J Phys Chem B. 112:13070-8.

Zweckstetter, M. (2008). NMR: prediction of molecular alignment from structure using the PALES software. Nat Protoc. 3:679-90. 



\section{Appendix}

\section{A Purification of hVDAC1}

\section{A.1 Immobilized metal ion affinity chromatography (IMAC)}

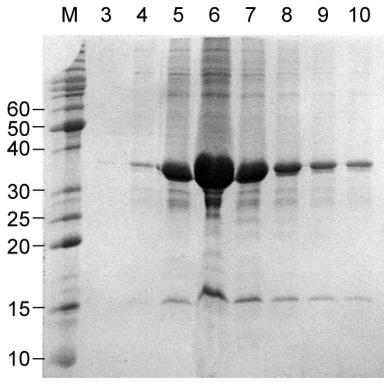

wt

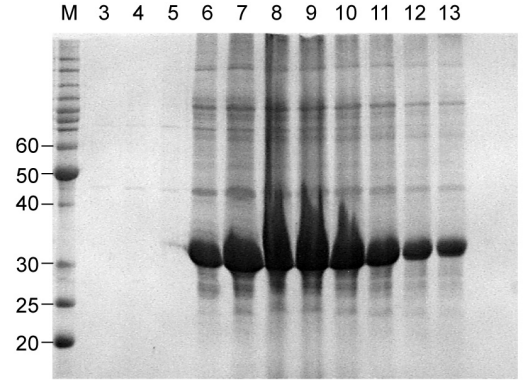

E73V

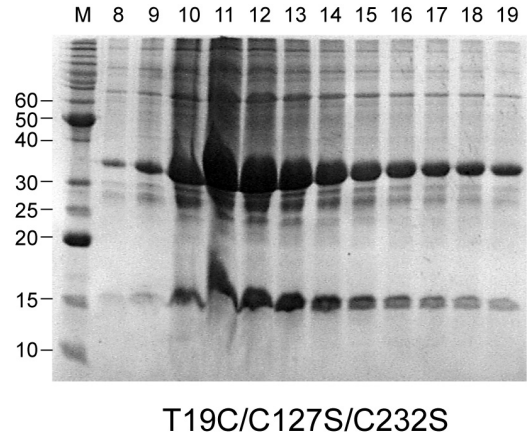

Supporting figure 1. Purification of hVDAC1 by IMAC. Fractions of hVDAC1 after purification by IMAC were subjected to SDS-PAGE and Coomassie Brilliant Blue staining. Purification of wt, E73V, and $\mathrm{T} 19 \mathrm{C} / \mathrm{C} 127 \mathrm{~S} / \mathrm{C} 232 \mathrm{~S}$ hVDAC1 are shown as examples. The marker (M) and arbitrary fraction numbers are indicated. The purity of other hVDAC1 mutants was similar. 


\section{A.2 Gel filtration}

A
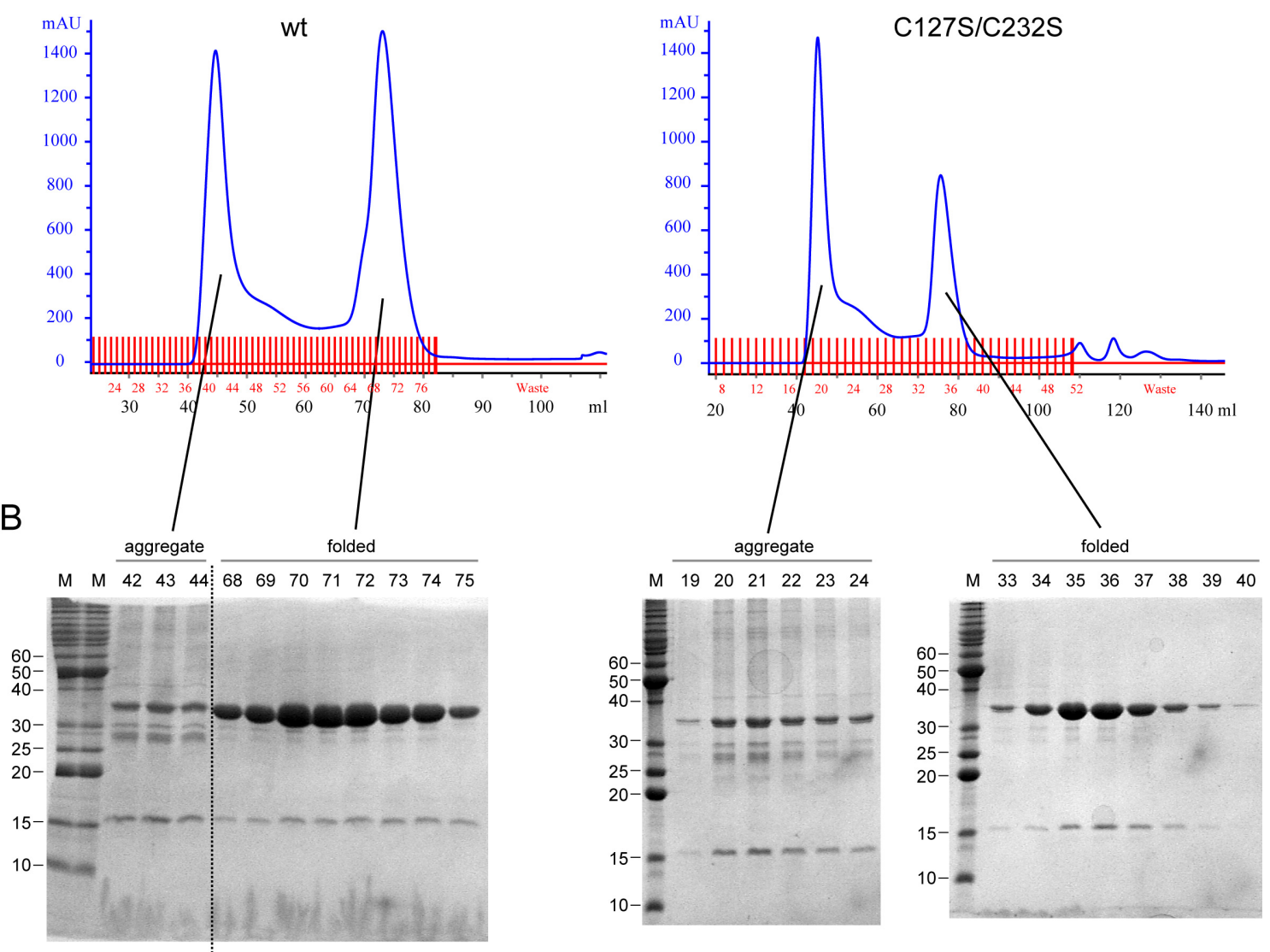

Supporting figure 2. Additional gel filtration purification step applied to some hVDAC1 preparations. (A) Examplary chromatograms of $w t$ and C127S/C232S hVDAC1 subjected to the gel filtration step. Two peaks corresponding to aggregated/unfolded hVDAC1 and folded monomeric hVDAC1 are visible. Fractions were subjected to SDS-PAGE and Coomassie Briliant Blue staining as depicted in (C). All other mutants that were purified by gel filtration showed similar chromatograms and purity of hVDAC1 containing fractions. 


\section{B Alignment of VDAC sequences}

Sequences of VDAC from various species, including hVDAC2 and hVDAC3, were aligned with ClustalW (standard settings) and visualized with Jalview (Supporting figure 3).

Supporting figure 3 (following on next page). Alignment of VDAC sequences. VDAC1 from Bos taurus (btVDAC1), Canis lupus familiaris (clVDAC1), Sus scrofa (domestic pig) (pVDAC1), Homo sapiens (hVDAC1), Mus musculus (mVDAC1), Rattus norvegicus (rVDAC1), Salmo salar (ssVDAC1), and VDAC from Xenopus laevis (xIVDAC), Drosophila melanogaster (dmVDAC), Caenorhabditis elegans (ceVDAC), Saccharomyces cerevisiae (scVDAC) and Neurospora crassa VDAC (ncVDAC), as well as human VDAC2 lacking $11 \mathrm{~N}$-terminal residues (hVDAC2-Nterm) and human VDAC3 (hVDAC3) were aligned with ClustalW and visualized with Jalview. 


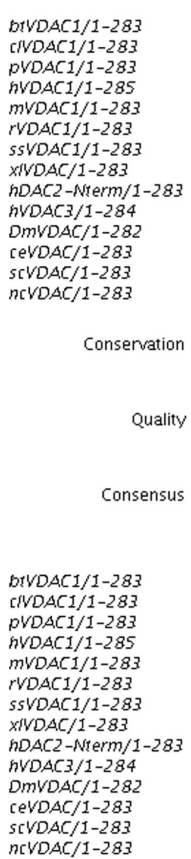

bIVDAC1/1-283 CNDAC1/1-283 PVDAC1/1-283 hVDAC1/1-285
$m V D A C 1 / 1-283$ IVDAC1/1-283 SSVDACI11-283 XVDAC/1-283 hDAC2-Nterm/1-283 nVDAC $3 / 1-284$ DIVDAC/1-282 CeVDAC/1-283 SCVDAC/1-283

Conservation

Quality

Consensus bTVDAC1/1-283 CMDAC1/1-283 PVDAC1 1 $1-283$
hVDAC1/1-285 mVDAC1/1-283 $m V D A C 1 / 1-283$
rVDAC1/1-283 SSVDAC1/1-283 XIVDAC/1-283 hDAC2-Nterm/1-283 hVDACB $/ 1-284$ DNDAC/1-282 CeVDAC/1-283 $n C V D A C / 1-283$

Conservation

Quality

Consensus DrVDAC1/1-283 CIVDAC1/1-283 nVDAC1/1-285 mVDAC1/1-283 IVDAC1/1-283 SSVDAC 1/1-283 XIVDAC/1-283 nDAC2-Nterm/1 DOVDAC/1-28 CeVDAC/1-283 scVDAC/1-283 $n \subset V D A C / 1-283$

Conservation

Quality

Consensus

DIVDAC1/1-283 WOAC1/1-283 DVDAC1/1-285 mVDAC1/1-283 IVDAC1/1-283 SSVDAC1 1 1-283 XIVDAC/1-283 hDAC2-Nterm $/ 1-2$ nVDAC3/1-284 OmVDAC/1-282 CeVDAC/1-283 nCVDAC/1-283

Consenvation

Quality

Consensus

$$
\begin{aligned}
& \\
& 144 \\
& 144 \\
& 144 \\
& 144 \\
& 144 \\
& 144 \\
& 144 \\
& 144 \\
& 144 \\
& 145 \\
& 143 \\
& 144 \\
& 142 \\
& 142
\end{aligned}
$$
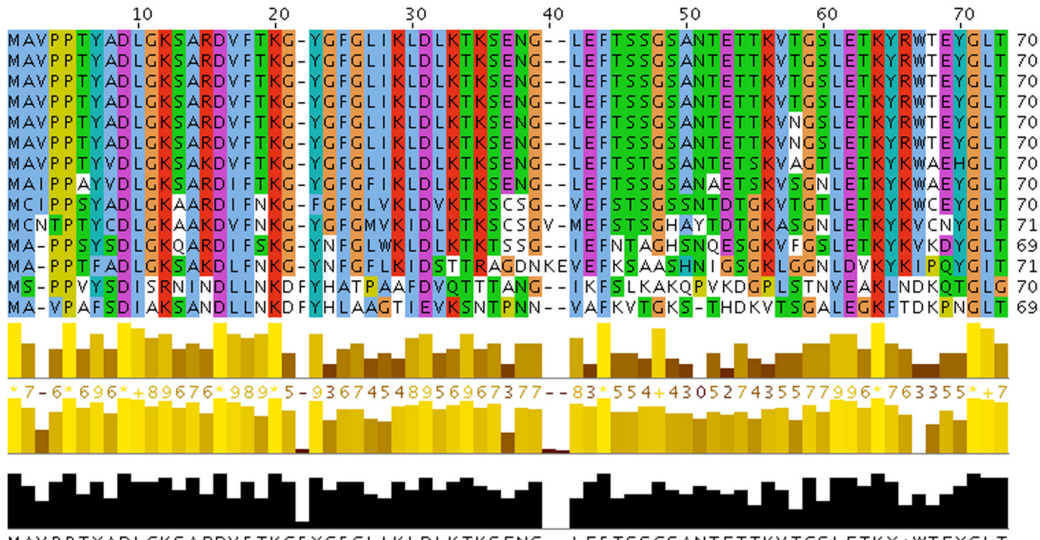

MAVPPTYADL GKSARDVF TKGFYGF GLI KLDLKTKS ENG - - LEF TSSCSANTETTKVT GSLETKY+WTEYGLT

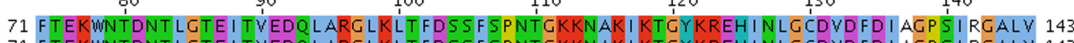
71 FTEKWNTDNT LGTE I TVEDOLARGLKLT FDS F S PNT CKKNAK I KT GYKREH INLGCDVDFD I AGP S I RGALV 143 71 FTEKWNTDNT LGTE I TVEDOLARG LKLTFDSSF SPNT GKKNAKI KTGYKREH I NLGCDMDFD I AGP S I RGALV 143 71 FTEKWWNTDNT LGTEI TVEDQLARG LKLT FDSS F SPNT GKKNAK I KT GYKREHINLGCDVDFDI AGP S I RGALV 143 71 FTEKWNTDNT LGTE I TVEDQLARG LKLTFDSSF SPNT CKKNAK I KTCYKREH INLCCDVDFD I AGP S I RGALV 143 71 F TEKWNTDNT LGTE I TLEDQLAKG LQFT FDSS F SPNT CKKSGK I KTCYKREH I NLGCDVDYD I NGTAVHGVAV 143 71 FTEKWNTDNT LGTE ITVEDQLAKGLKLT FDSSF SPNTGKKNAKVKSAYKREHLNVGCDMDFDI AGP SVRGAVV 143 71 FTEKINTDNT LCTE I AI EDQ I CQGLKLT FDTTF SPNT CKKSCK I KS S YKREC INLCCDVDFDFACP AI HCSAV 143

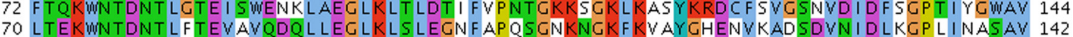
72 LTEKWNTENQLGTVI EVNEO FGRG LKVT LDSLYAP HACKRSCKVKLDWALPTARVT ADVGVT - S APV INAAGV 143 71 LTOGWSNTNNLOTKLEFAN-LTPGLKNELIT SLTPGVAK-SAVLNTTFTEP F FTARGAFDLCLKSPT FVGDLT 141 7O VTOTWNT ANALET KVEMADN LAKG LKAEC I FSFLPATNARCAKFN LHFKOSNFHGRAF FDL-LKGPTAN IDAI 141
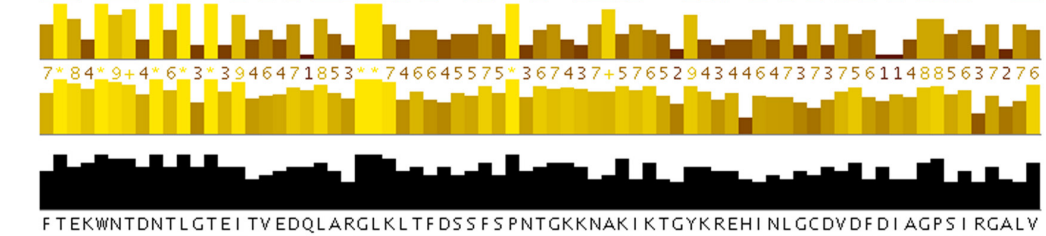
150
160
170
180
190
200

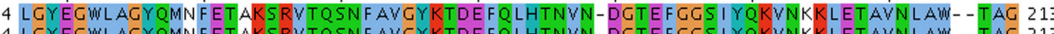
144 LCYECWLAGYOMNFETAKSRVTOSNFAVGKTDEFOLHTNVN-DCTEFGGS I YOKVNKKLETAVNLAWN- - TAG 213

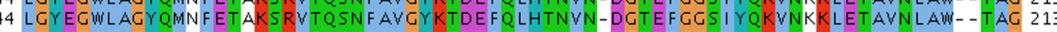
作 144 LCYECWLACYQMNFETS KSRVTQSN FAVCYKTDE FQLHTNVN-DCTEF GGS I YQKVNKKLETAVNLAW- - TAC 213 144 VCYECWLAGYQMTF EACKNRVTQSNFAVGYKTDEFQLHTNVN-DGTEFGCS I YQKVNDQLETAVN LAW- - TAC 213

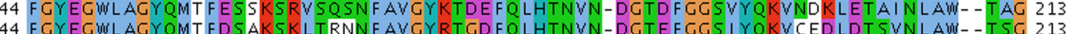
145 LAFEG WLAGYQMS FDTAKSKLSQNNFALGYKAAD FQLHTHVN-DGTEFGGS I YQKVNEK I ET I I LLAWN- - TAC 214

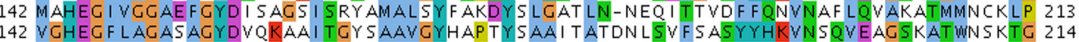

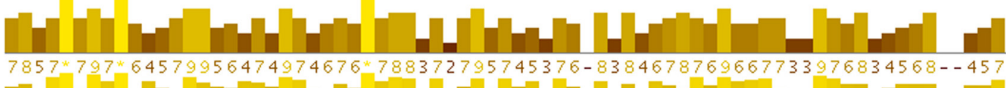
$7857-79704579956474974676 \div 88372795745376-8384678769667733976834568-057$

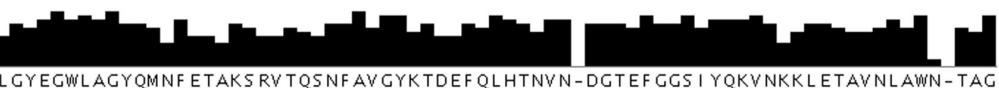

LCYECWLACYQMNF ETAKS RVTQSNF AVCYKTDEF QLHTNVN-DGTEF GCSI YOKVNKKLETAVN

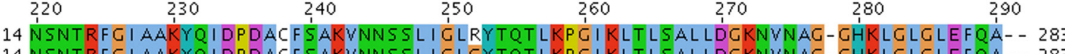
214 NSNTRFGI AAKYQ IDPDACF SAKVNNSSL GLGTTQTKPGI 214 NSNTRFGIAAKYOIDPDACF SAKYNNS SLIGLGYTOTLKPGIKLTLSALLDGKNVNAG-GHKLGLCLEFOARS 285 214 NSNTRFG I AAKYOVDPDACF 5 AKVNNS SLI GLGYTOT LKPG I KLTLSALLDGKNVNAG-GHKLGLGLEFOA- 283 214 NSNTRFG I AAKYOVDPDACF 5 AKYNNS SLI GLGYTOT LKPGI KLTLSALLDGKNYNAG-GHKLGLGLEFOA- 283 214 NSNTRFG I AAKYQ I DADAAF SAKVNNS 5 LVGLGYTQT LKPG I KLT LSAL LDGKN INAG-GHKLGLGLEFEA- - 283 214 NSNTRF I AAKYQI D SDASF SAKVNNS S I I L LGTQT LKP I I LT LSTLVDGKN INAC-GHKLGLGLEFEA- - 283 214 TNCTRF I I AAKYQLDPTAS I SAKVNNS S L I GVGYTQT LRP GVKLT L SALVDGKS I NAG-CHKVGLALELEA-- 283 215 SNNTRF G I AAKYMLDCRT S L SAKVNNAS L I GLCYTQT LRP CVKLT LSAL IDCKNF SAG-CHKVGLCFELEA-- 284 213 TSNTKFAI GAKYQLDDDASVRAKVNNASQVG LGYQRLRDGVTLT LST LVDGKNFNAC-GHK I GVGLELEA-- 282 N 214 NSNVN I EFATRY LPDAS SQVKAKVSDSG IVT LAYKQLLRPGVTLCVGSSFDALKLSEP-VHKLGWSLS FDA- - 283
215 NT-VGLEVATKYRIDPVSFVKGKINDRGVAAIAVNVLLREGVTLCVCASFDTQKLDQA-THKVGTSFTFES-- 283

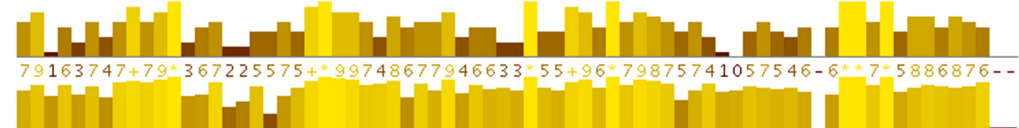

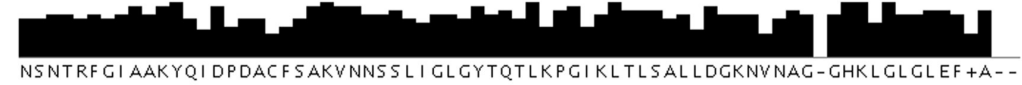

Supporting figure 3. Alignment of VDAC sequences. 


\section{Chemical shift assignment of hVDAC1}

Supporting table 1. Backbone chemical shifts of $w t$ hVDAC1.

\begin{tabular}{|c|c|c|c|c|c|c|c|c|c|c|}
\hline \multirow[b]{2}{*}{ residue } & \multicolumn{5}{|c|}{ Previous assignment (Bayrhuber et al. 2008) } & \multicolumn{5}{|c|}{ Updated assignment } \\
\hline & CAi & CAi-1 & COi-1 & $\mathbf{H i}$ & $\mathbf{N}$ & CAi & CAi-1 & COi-1 & $\mathbf{H i}$ & $\mathbf{N}$ \\
\hline $\mathrm{T} 6$ & 60.24 & 61.09 & 174.4 & 7.413 & 106.2 & 60.46 & 61.35 & 174.4 & 7.410 & 106.2 \\
\hline Y7 & 62.03 & 60.26 & 176.2 & 7.653 & 122.5 & 62.31 & 60.49 & 176.2 & 7.649 & 122.5 \\
\hline A8 & 53.57 & 61.98 & - & 8.405 & 119.0 & 53.81 & 62.11 & 178.6 & 8.393 & 119.1 \\
\hline D9 & 53.55 & - & 177.7 & 7.215 & 115.2 & 53.81 & - & 177.7 & 7.219 & 115.2 \\
\hline L10 & 55.90 & 53.51 & 177.5 & 7.354 & 125.4 & 56.18 & 53.82 & 177.4 & 7.349 & 125.3 \\
\hline K12 & - & - & - & - & - & 59.82 & 44.85 & 175.5 & 7.273 & 122.4 \\
\hline A14 & 54.81 & - & - & 8.317 & 122.0 & 55.13 & 62.98 & - & 8.289 & 121.9 \\
\hline R15 & 59.80 & 54.89 & - & 7.281 & 117.3 & 60.00 & 55.06 & - & 7.288 & 117.3 \\
\hline D16 & - & - & - & - & - & 57.10 & - & - & 8.528 & 118.6 \\
\hline V17 & - & - & - & - & - & 66.65 & 57.20 & - & 7.482 & 120.4 \\
\hline K20 & - & - & - & 7.824 & 122.1 & 60.82 & 56.21 & - & 7.843 & 122.1 \\
\hline Y22 & 55.82 & 44.24 & 171.6 & 6.591 & 113.7 & 56.04 & 44.43 & - & 6.593 & 113.7 \\
\hline G23 & 46.01 & 55.89 & 175.9 & 8.667 & 105.3 & - & - & - & - & - \\
\hline F24 & 56.09 & - & - & 7.067 & 120.4 & 56.47 & 45.74 & - & 7.062 & 120.2 \\
\hline G25 & 45.26 & 56.16 & - & 8.140 & 110.3 & 45.48 & 56.53 & - & 8.126 & 110.2 \\
\hline L26 & 53.04 & 45.28 & 172.9 & 7.472 & 118.4 & 53.28 & 45.48 & 172.9 & 7.470 & 118.5 \\
\hline I 27 & 59.80 & 53.05 & 176.7 & 8.671 & 120.9 & 60.04 & 53.15 & 176.6 & 8.671 & 120.9 \\
\hline K28 & 54.83 & 59.78 & 175.0 & 8.748 & 126.9 & 55.05 & 59.99 & 175.0 & 8.744 & 126.9 \\
\hline L29 & 53.31 & 54.89 & 174.0 & 8.708 & 124.2 & 53.55 & 55.14 & 174.0 & 8.712 & 124.3 \\
\hline D30 & - & - & - & - & - & 53.74 & - & 174.4 & 8.461 & 123.2 \\
\hline L31 & 53.69 & - & 173.9 & 9.103 & 123.9 & 53.92 & - & 173.9 & 9.097 & 123.9 \\
\hline K32 & 55.40 & - & - & 8.075 & 125.1 & 55.19 & 53.94 & - & 8.603 & 125.5 \\
\hline T33 & 60.11 & 55.16 & - & 9.023 & 116.0 & 60.36 & 55.30 & 172.2 & 9.009 & 115.9 \\
\hline K34 & 55.03 & 60.18 & - & 8.164 & 123.8 & 55.33 & 60.28 & 172.8 & 8.109 & 123.7 \\
\hline S35 & - & - & - & - & - & 57.13 & 55.12 & 175.8 & 8.808 & 120.2 \\
\hline G38 & - & - & - & - & - & 45.43 & 52.94 & - & 7.848 & 106.6 \\
\hline L39 & - & - & - & - & - & 55.22 & 45.32 & - & 7.586 & 122.5 \\
\hline E40 & - & - & - & - & - & 55.10 & - & 176.0 & 8.811 & 124.5 \\
\hline F41 & - & - & - & - & - & 56.76 & - & - & 8.756 & 124.6 \\
\hline T42 & 61.19 & 56.57 & - & 8.878 & 120.6 & 61.48 & 56.94 & - & 8.871 & 120.7 \\
\hline S43 & 56.32 & 61.11 & - & 9.268 & 122.0 & 56.61 & 61.41 & - & 9.256 & 122.0 \\
\hline S44 & 56.47 & - & - & 8.629 & 120.5 & 56.69 & - & 172.9 & 8.618 & 120.5 \\
\hline G45 & 44.92 & 56.16 & 173.2 & 9.106 & 111.5 & 44.92 & 56.58 & 173.2 & 9.103 & 111.6 \\
\hline S46 & 56.66 & 44.88 & 172.0 & 9.074 & 116.0 & 56.87 & 45.05 & 172.0 & 9.070 & 116.0 \\
\hline A47 & 49.43 & - & 172.2 & 9.150 & 123.9 & 49.63 & 56.98 & 172.3 & 9.151 & 123.9 \\
\hline $\mathrm{N} 48$ & 52.58 & 49.32 & 175.3 & 8.307 & 122.5 & 52.86 & 49.66 & 175.3 & 8.304 & 122.4 \\
\hline K53 & - & - & - & - & - & - & 64.13 & 172.2 & 7.508 & 120.4 \\
\hline V54 & 59.76 & - & 176.7 & 7.511 & 120.5 & 59.99 & 56.60 & 176.7 & 8.589 & 125.3 \\
\hline T55 & 59.79 & - & 175.3 & 8.743 & 118.6 & 60.02 & 60.03 & 175.2 & 8.744 & 118.7 \\
\hline G56 & 45.31 & 59.75 & - & 8.299 & 109.6 & 45.47 & 60.02 & - & 8.292 & 109.5 \\
\hline S57 & 57.27 & 45.31 & - & 9.085 & 113.1 & 57.39 & 45.61 & - & 9.140 & 113.5 \\
\hline L58 & 54.14 & 57.28 & 172.4 & 9.137 & 122.4 & 54.28 & 57.58 & 172.4 & 9.129 & 122.4 \\
\hline E59 & 54.59 & 53.86 & - & 9.014 & 123.5 & 54.84 & 54.10 & - & 9.037 & 124.7 \\
\hline T60 & - & - & - & - & - & 61.05 & 54.85 & - & 9.027 & 120.6 \\
\hline K61 & 54.47 & 60.93 & 172.7 & 8.923 & 126.9 & 54.68 & 61.06 & 172.7 & 8.914 & 127.0 \\
\hline
\end{tabular}




\begin{tabular}{|c|c|c|c|c|c|c|c|c|c|c|}
\hline Y62 & - & - & - & - & - & 56.94 & 54.83 & - & 9.337 & 125.2 \\
\hline R63 & - & - & - & - & - & 55.40 & 57.27 & - & 8.071 & 125.1 \\
\hline W64 & - & - & - & - & - & 53.59 & 54.54 & - & 8.924 & 126.4 \\
\hline Y67 & - & - & - & - & - & 56.41 & 59.34 & 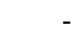 & 6.565 & 114.3 \\
\hline G68 & 46.59 & 55.95 & 178.2 & 7.555 & 105.1 & 46.87 & 56.13 & - & 7.557 & 05.2 \\
\hline L69 & 53.84 & 46.74 & 175.8 & 7.334 & 120.5 & 54.03 & 46.96 & 175.7 & 7.330 & 120.5 \\
\hline $\mathrm{T} 70$ & 62.05 & - & 176.6 & 9.085 & 120.6 & 62.31 & 54.05 & 176.6 & 9.071 & 120.7 \\
\hline F71 & 55.90 & 62.13 & 172.4 & 9.310 & 128.1 & 56.23 & 62.34 & 172.4 & 9.304 & 128.1 \\
\hline $\mathrm{T} 72$ & 61.28 & 55.65 & - & 9.017 & 120.8 & 61.53 & 55.72 & 174.0 & 9.016 & 120.7 \\
\hline V73 & 60.52 & - & - & 8.976 & 125.6 & 60.84 & 61.55 & 173.6 & 8.978 & 125.7 \\
\hline K74 & 54.42 & 60.52 & - & 9.106 & 125.9 & 54.67 & 60.71 & 173.6 & 9.107 & 125.8 \\
\hline W75 & 55.42 & - & - & 9.094 & 124.1 & 55.67 & 54.48 & - & 9.093 & 124.2 \\
\hline N76 & 51.29 & 55.37 & - & 7.622 & 123.1 & 51.47 & 55.73 & 174.8 & 7.479 & 122.6 \\
\hline $\mathrm{T} 77$ & 63.64 & 51.29 & - & 8.058 & 108.0 & 63.88 & 51.63 & - & 8.005 & 108.5 \\
\hline D78 & 53.93 & 63.66 & - & 7.650 & 121.0 & 54.17 & 63.75 & - & 7.710 & 121.2 \\
\hline N79 & 53.94 & - & - & 8.504 & 115.0 & 54.31 & 54.17 & 176.0 & 8.430 & 114.8 \\
\hline T80 & 62.54 & 53.97 & - & 7.259 & 112.8 & 62.97 & 54.21 & 174.1 & 7.318 & 113.4 \\
\hline L81 & 53.33 & 62.76 & 173.7 & 8.597 & 127.5 & 53.54 & 62.99 & 173.7 & 8.591 & 127.5 \\
\hline G82 & & & & & & 44.58 & 53.20 & 175.8 & 9.367 & 112.5 \\
\hline T83 & & & & & & 59.80 & 44.57 & 172.6 & 8.803 & 114.2 \\
\hline E84 & & & & & & 54.65 & 59.75 & - & 8.880 & 124.7 \\
\hline I 85 & 54.33 & 54.49 & - & 9.175 & 127.3 & 60.15 & 54.33 & - & 9.168 & 127.3 \\
\hline T86 & 60.77 & 59.82 & 174.5 & 9.068 & 123.7 & 61.03 & 60.11 & 174.5 & 9.063 & 123.7 \\
\hline V87 & 59.95 & 60.85 & 173.0 & 9.300 & 124.4 & 60.16 & 61.03 & 173.1 & 9.301 & 124.5 \\
\hline E88 & 54.88 & - & - & 8.765 & 124.5 & - & - & - & 9.289 & 129.4 \\
\hline D89 & - & - & - & - & - & 56.96 & 55.40 & - & 8.704 & 118.3 \\
\hline Q90 & 55.24 & 57.14 & 173.0 & 8.708 & 118.3 & 55.49 & 57.18 & 175.2 & 7.826 & 116.6 \\
\hline L91 & - & - & - & - & - & $\mathbf{5 8 . 5 3}$ & 55.42 & - & 8.200 & 117.8 \\
\hline R93 & - & - & - & - & - & 57.88 & 58.62 & - & 8.825 & 124.1 \\
\hline G94 & 45.09 & 57.69 & 177.2 & 9.391 & 115.5 & 45.31 & 57.87 & 177.2 & 9.391 & 115.5 \\
\hline L95 & 53.66 & 45.04 & 173.7 & 7.898 & 122.8 & 53.92 & 45.26 & 173.7 & 7.896 & 122.8 \\
\hline K96 & 55.08 & 53.81 & 175.3 & 9.676 & 132.5 & 55.31 & 54.08 & 175.3 & 9.670 & 132.4 \\
\hline L97 & 53.54 & - & - & - & - & 53.82 & 55.20 & - & 8.694 & 127.2 \\
\hline T98 & 61.15 & 53.53 & 174.5 & 9.133 & 120.5 & 61.40 & 53.76 & 174.5 & 9.135 & 120.5 \\
\hline F99 & 56.05 & 61.16 & 172.8 & 9.449 & 127.1 & 56.19 & 61.42 & 173.0 & 9.445 & 127.1 \\
\hline D100 & 52.23 & 56.09 & 173.0 & 8.630 & 129.0 & 52.45 & 56.40 & 173.0 & 8.631 & 129.0 \\
\hline S101 & 57.07 & 52.20 & - & 8.686 & 118.8 & 56.70 & 52.46 & 174.5 & 8.744 & 119.2 \\
\hline $\mathrm{S} 102$ & 57.07 & - & - & 8.686 & 118.8 & 57.29 & 56.84 & - & 8.685 & 118.9 \\
\hline F103 & 55.80 & 57.07 & - & - & - & 56.01 & 57.54 & - & 8.912 & 122.5 \\
\hline S104 & - & - & - & - & - & 53.24 & 56.04 & - & 7.808 & 120.8 \\
\hline G108 & 45.70 & 60.02 & - & 9.377 & 112.8 & 45.52 & - & - & 8.219 & 112.0 \\
\hline K109 & 56.39 & 45.45 & 173.7 & 7.450 & 120.5 & 56.62 & 45.67 & 173.7 & 7.450 & 120.5 \\
\hline K110 & 55.38 & 56.53 & 173.8 & 8.804 & 129.5 & 55.00 & 56.61 & 175.8 & 8.451 & 124.6 \\
\hline N111 & - & - & - & - & - & 52.84 & 55.02 & 175.2 & 8.569 & 120.3 \\
\hline A112 & - & - & - & - & - & 51.68 & - & - & 8.559 & 124.9 \\
\hline K113 & 54.56 & 51.35 & - & 9.007 & 119.8 & 54.86 & 51.65 & - & 8.997 & 119.9 \\
\hline I114 & 59.34 & 54.75 & 177.8 & 8.779 & 120.5 & 59.49 & 54.91 & 175.7 & 8.782 & 120.5 \\
\hline K115 & 54.63 & 59.29 & 175.0 & 9.269 & 126.8 & 54.86 & 59.53 & 174.9 & 9.265 & 126.8 \\
\hline T116 & 60.11 & 54.63 & 175.8 & 8.679 & 117.2 & 60.36 & 54.87 & 175.8 & 8.677 & 117.2 \\
\hline G117 & 44.47 & 60.11 & 174.5 & 9.155 & 113.8 & 44.70 & 60.43 & 174.5 & 9.149 & 113.7 \\
\hline Y118 & 56.56 & 44.42 & 171.1 & 9.131 & 123.6 & 56.77 & 44.67 & 171.1 & 9.134 & 123.7 \\
\hline K119 & 52.87 & 56.48 & 172.2 & 7.583 & 124.9 & 53.10 & 56.75 & 172.3 & 7.590 & 125.0 \\
\hline
\end{tabular}




\begin{tabular}{|c|c|c|c|c|c|c|c|c|c|c|}
\hline $\mathrm{R} 120$ & 53.77 & 52.85 & 172.9 & 8.700 & 117.7 & 53.94 & 53.12 & 173.0 & 8.703 & 117.9 \\
\hline E121 & - & - & - & - & - & 60.41 & 53.88 & 174.5 & 9.063 & 120.8 \\
\hline E121* & - & - & - & - & - & 59.72 & 54.14 & - & 8.453 & 120.8 \\
\hline H122 & - & - & - & - & - & 60.31 & 60.38 & 177.1 & 7.869 & 111.7 \\
\hline $\mathrm{H} 122 *$ & - & - & - & - & - & 59.75 & - & - & 7.862 & 112.4 \\
\hline I123 & 59.85 & - & 173.5 & 7.893 & 118.0 & 60.12 & - & 173.5 & 7.903 & 118.0 \\
\hline N124 & 52.78 & 59.92 & 171.9 & 8.964 & 125.4 & 52.98 & 60.14 & 171.9 & 8.961 & 125.4 \\
\hline L125 & 53.25 & 52.76 & 174.5 & 9.488 & 128.9 & 53.48 & 53.03 & 174.5 & 9.484 & 128.9 \\
\hline G126 & 44.79 & 53.27 & 175.8 & 9.480 & 113.6 & 45.01 & 53.47 & 175.8 & 9.478 & 113.6 \\
\hline C127 & 58.10 & 44.78 & 171.9 & 8.525 & 121.3 & 58.31 & 44.99 & 172.0 & 8.524 & 121.3 \\
\hline D128 & 52.42 & 58.14 & 172.7 & 9.370 & 130.0 & 52.62 & 58.34 & 172.7 & 9.367 & 130.0 \\
\hline M129 & 52.44 & 52.40 & 174.9 & 9.396 & 123.1 & 54.62 & 52.58 & 174.9 & 9.391 & 123.1 \\
\hline D130 & 52.75 & 54.40 & 173.0 & 8.545 & 125.0 & 52.98 & 54.60 & 173.0 & 8.537 & 125.0 \\
\hline F131 & 57.94 & 52.74 & 176.1 & 8.501 & 123.0 & 58.15 & 52.94 & 176.0 & 8.500 & 123.0 \\
\hline D132 & 54.10 & 57.82 & - & 7.378 & 122.9 & 54.38 & 58.12 & 174.7 & 7.398 & 122.9 \\
\hline I133 & 63.06 & 53.88 & - & 8.442 & 124.0 & 63.26 & 54.26 & - & 8.408 & 124.4 \\
\hline A134 & 52.42 & 63.01 & 176.8 & 8.466 & 123.4 & 52.65 & 63.29 & 176.8 & 8.461 & 123.4 \\
\hline G135 & 44.50 & 52.42 & 178.0 & 7.671 & 106.3 & 44.73 & 52.63 & 177.9 & 7.667 & 106.3 \\
\hline S137 & 57.34 & 62.93 & 177.2 & 8.819 & 118.2 & 57.57 & 63.17 & 177.1 & 8.821 & 118.2 \\
\hline I138 & 59.57 & 57.29 & 172.7 & 9.087 & 122.5 & 59.82 & 57.52 & 172.7 & 9.085 & 122.6 \\
\hline R139 & 53.83 & 59.62 & 173.3 & 9.030 & 128.5 & 54.05 & 59.84 & 173.4 & 9.027 & 128.5 \\
\hline G140 & 43.84 & 53.84 & 176.1 & 8.726 & 113.4 & 44.05 & 54.05 & 176.1 & 8.729 & 113.4 \\
\hline A141 & 50.83 & 43.80 & 170.7 & 8.934 & 124.0 & 51.08 & 44.02 & 170.8 & 8.935 & 124.0 \\
\hline L142 & 53.61 & 50.87 & 174.9 & 9.231 & 123.9 & 53.82 & 51.05 & 174.9 & 9.224 & 123.9 \\
\hline V143 & 60.93 & 53.63 & 174.4 & 9.014 & 125.2 & 61.18 & 53.85 & 172.0 & 9.007 & 125.2 \\
\hline L144 & 51.99 & 60.91 & 174.7 & 9.346 & 126.2 & 52.21 & 61.18 & 174.7 & 9.344 & 126.2 \\
\hline G145 & 44.65 & 51.93 & 176.2 & 8.139 & 105.2 & 44.88 & 52.23 & 176.2 & 8.145 & 105.3 \\
\hline Y146 & 59.11 & 44.70 & 172.9 & 8.586 & 122.4 & 59.31 & 44.90 & 173.0 & 8.588 & 122.4 \\
\hline Y146* & - & - & - & - & - & 59.18 & 45.04 & - & 8.475 & 122.9 \\
\hline E147 & 56.93 & 59.13 & 173.6 & 9.202 & 125.6 & 57.13 & 59.33 & 173.7 & 9.199 & 125.6 \\
\hline E147* & - & - & - & - & - & 57.45 & 59.21 & - & 9.091 & 126.0 \\
\hline G148 & 45.10 & 57.04 & 175.3 & 8.011 & 108.1 & 45.31 & 57.30 & 175.3 & 8.012 & 108.1 \\
\hline G148* & - & - & - & - & - & 45.48 & - & - & 8.110 & 108.8 \\
\hline W149 & 57.19 & 45.05 & 173.3 & 8.102 & 121.5 & 57.39 & 45.35 & 173.3 & 8.109 & 121.5 \\
\hline W149* & - & - & - & - & - & 57.50 & 45.40 & - & 8.155 & 121.5 \\
\hline L150 & 53.47 & 57.21 & 177.4 & 8.955 & 123.4 & 53.71 & 57.45 & 177.3 & 8.954 & 123.4 \\
\hline A151 & 50.69 & 53.47 & 175.4 & 8.863 & 121.7 & 50.89 & 53.71 & 175.4 & 8.859 & 121.7 \\
\hline G152 & 45.26 & 50.70 & 175.4 & 9.626 & 107.8 & 45.48 & 50.89 & 175.4 & 9.621 & 107.8 \\
\hline Y153 & 55.95 & 45.26 & 171.2 & 9.203 & 122.2 & 56.19 & 45.49 & 171.2 & 9.201 & 122.2 \\
\hline Q154 & 53.10 & 55.97 & 171.9 & 8.210 & 127.9 & 53.32 & 56.20 & 172.0 & 8.209 & 128.0 \\
\hline M155 & 52.87 & - & 172.2 & 8.740 & 121.3 & 53.12 & - & 172.2 & 8.739 & 121.3 \\
\hline N156 & 52.24 & - & 174.3 & 7.961 & 119.4 & 52.46 & 53.11 & 174.3 & 7.966 & 119.4 \\
\hline F157 & 56.17 & 52.25 & 175.4 & 9.544 & 127.7 & 56.39 & 52.43 & 173.4 & 9.543 & 127.7 \\
\hline E158 & 54.85 & 56.17 & 173.5 & 8.041 & 127.7 & 55.08 & 56.41 & 173.5 & 8.046 & 127.8 \\
\hline T159 & - & - & - & - & - & 65.12 & 55.09 & 177.1 & 7.632 & 117.1 \\
\hline A160 & - & - & - & - & - & 54.75 & 65.08 & 177.1 & 9.135 & 125.0 \\
\hline S162 & 58.37 & 54.58 & 175.3 & 7.470 & 114.7 & 58.57 & 54.80 & 175.2 & 7.470 & 114.7 \\
\hline R163 & 53.72 & 58.36 & 173.1 & 6.802 & 115.0 & 53.97 & 58.64 & 173.1 & 6.812 & 115.1 \\
\hline V164 & - & - & - & - & - & 62.27 & 53.94 & 174.5 & 8.800 & 124.4 \\
\hline T165 & - & - & - & - & - & 61.58 & 62.21 & 177.0 & 8.624 & 118.9 \\
\hline Q166 & - & - & - & - & - & 55.52 & 61.30 & 174.6 & 7.428 & 121.1 \\
\hline S167 & 57.03 & 55.29 & 174.6 & 8.324 & 119.0 & 57.27 & 55.52 & 174.6 & 8.323 & 119.0 \\
\hline
\end{tabular}




\begin{tabular}{|c|c|c|c|c|c|c|c|c|c|c|}
\hline N168 & 51.49 & 57.08 & 172.6 & 9.113 & 127.2 & 51.70 & 57.29 & 172.7 & 9.106 & 127.2 \\
\hline F169 & 56.05 & 51.53 & 171.9 & 8.606 & 118.3 & 56.29 & 51.69 & 171.9 & 8.604 & 118. \\
\hline A170 & 50.98 & 56.07 & 173.4 & 8.819 & 122.7 & 51.21 & 56.26 & 173.4 & 8.817 & 127 \\
\hline V171 & 60.11 & 50.98 & 176.2 & 8.215 & 116.8 & 60.37 & 51.20 & 176.2 & 8.215 & 16. \\
\hline G172 & 45.66 & 60.13 & 175.5 & 9.415 & 113.0 & 45.89 & 60.37 & 175.5 & 9.418 & 113. \\
\hline Y173 & 56.54 & 45.65 & 170.0 & 8.582 & 119.5 & 56.78 & 45.88 & 170.1 & 8.582 & 119 \\
\hline K174 & - & - & - & - & - & 55.58 & 56.73 & 173.8 & 8.802 & 129. \\
\hline K174s & - & - & - & - & - & 55.80 & 57.12 & - & 8.757 & 129 \\
\hline $\mathrm{T} 175$ & - & - & - & - & - & 59.71 & 55.66 & 174.0 & 8.580 & 117. \\
\hline $\mathrm{T} 175 \mathrm{~s}$ & - & - & - & - & - & 59.86 & 55.62 & - & 8.618 & 118 \\
\hline F178 & - & - & - & - & - & 56.54 & - & 173.9 & 7.508 & 20. \\
\hline Q179 & - & - & - & - & - & 53.94 & 56.45 & 174.2 & 8.687 & 122. \\
\hline L180 & - & - & 173.9 & 9.353 & 127.8 & 53.69 & - & 173.9 & 9.342 & 127.8 \\
\hline H181 & 55.08 & - & - & 9.140 & 128.7 & - & 53.47 & - & 9.140 & 128 \\
\hline N183 & 53.06 & 60.02 & 171.8 & 8.857 & 116.6 & 53.29 & 60.26 & 171.8 & 8.852 & 116. \\
\hline V184 & 60.04 & 52.92 & 172.6 & 8.918 & 118.4 & 60.25 & 53.16 & 172.7 & 8.918 & 118 \\
\hline N185 & 51.30 & 60.02 & 175.0 & 9.315 & 129.3 & 51.48 & 60.24 & 175.0 & 9.310 & 129.3 \\
\hline D186 & - & - & - & - & - & 56.11 & 51.50 & 174.9 & 9.023 & 125. \\
\hline G187 & - & - & - & - & - & 46.22 & 56.48 & 175.9 & 8.639 & 105. \\
\hline T188 & - & - & - & - & - & 62.64 & 46.41 & 173.3 & 7.629 & 109.1 \\
\hline E189 & - & - & - & - & - & 54.92 & 62.66 & - & 7.631 & 122.8 \\
\hline F190 & 55.79 & 54.46 & 174.3 & 8.876 & 125.8 & 56.01 & 54.66 & 174.3 & 8.870 & $125^{\circ}$ \\
\hline G191 & 44.48 & 55.79 & 175.7 & 8.533 & 110.4 & 44.68 & 56.00 & 175.7 & 8.529 & 110. \\
\hline G192 & 45.44 & 44.46 & 171.6 & 8.628 & 105.7 & 45.67 & 44.72 & 171.6 & 8.624 & 105.7 \\
\hline S193 & 56.02 & 45.48 & 171.4 & 9.751 & 114.7 & 56.38 & 45.79 & 171.4 & 9.752 & 114.7 \\
\hline I194 & 56.48 & 56.09 & 174.6 & 8.752 & 119.3 & - & 56.47 & 173.3 & 8.767 & 19.1 \\
\hline Y195 & 55.59 & 59.67 & 173.4 & 8.764 & 127.4 & 55.82 & 59.81 & 173.4 & 8.768 & 127. \\
\hline Q196 & 53.63 & 55.53 & 172.9 & 8.252 & 125.6 & 53.87 & 55.78 & 172.9 & 8.253 & 125.6 \\
\hline K197 & - & - & - & - & - & 55.91 & 53.84 & 174.5 & 8.892 & 130.4 \\
\hline V198 & - & - & - & - & - & 65.19 & 55.92 & 176.2 & 8.576 & 131. \\
\hline N199 & - & - & - & - & - & 52.27 & - & - & 8.146 & 113.6 \\
\hline E203 & - & - & - & - & - & 54.41 & - & - & 8.832 & 125.6 \\
\hline T204 & 59.45 & 54.02 & 174.8 & 8.387 & 112.2 & 59.66 & 54.26 & 174.8 & 8.383 & 112.2 \\
\hline A205 & 50.75 & 59.43 & 171.6 & 9.023 & 122.6 & 50.95 & 59.57 & 171.6 & 9.022 & 122. \\
\hline V206 & 59.49 & 50.73 & - & 8.958 & 116.9 & 59.78 & 50.92 & 174.6 & 8.952 & 116.9 \\
\hline N207 & 51.98 & 59.42 & 174.4 & 8.863 & 122.6 & 52.17 & 59.63 & 174.4 & 8.863 & 122.7 \\
\hline L208 & 54.14 & 51.91 & 172.5 & 8.703 & 121.7 & 54.35 & 52.14 & 172.6 & 8.700 & 121.6 \\
\hline A209 & 51.81 & 54.14 & 175.2 & 9.188 & 126.4 & 52.05 & 54.36 & 175.2 & 9.180 & 126. \\
\hline W210 & 57.62 & 51.80 & 175.4 & 8.976 & 121.3 & 57.85 & 52.05 & 175.4 & 8.977 & 121.4 \\
\hline $\mathrm{T} 211$ & 60.34 & 57.77 & 173.2 & 8.505 & 120.6 & 60.59 & 57.85 & 173.3 & 8.502 & 120.5 \\
\hline A 212 & 53.22 & 60.34 & 173.7 & 8.669 & 127.2 & 53.41 & 60.59 & 173.5 & 8.653 & 127.0 \\
\hline $\mathrm{G} 213$ & - & 53.20 & - & 8.412 & 110.2 & 45.26 & 53.59 & - & 8.395 & 110.2 \\
\hline $\mathrm{N} 214$ & - & - & - & - & - & 52.53 & 45.30 & - & 7.955 & 119.3 \\
\hline N216 & 53.00 & $\mathbf{5 8 . 9 0}$ & 174.3 & 8.353 & 120.4 & 53.21 & 59.35 & 174.3 & 8.353 & 120.4 \\
\hline $\mathrm{T} 217$ & 62.70 & 53.04 & 174.8 & 8.379 & 119.4 & 62.91 & 53.19 & 174.8 & 8.367 & 119. \\
\hline R218 & 54.96 & 62.63 & 174.5 & 9.038 & 125.7 & 55.19 & 62.90 & 174.2 & 9.031 & 125.7 \\
\hline F219 & 55.80 & 54.91 & 175.1 & 8.028 & 119.2 & 56.03 & 55.14 & 175.1 & 8.027 & 119.2 \\
\hline $\mathrm{G} 220$ & 44.99 & 55.86 & 173.5 & 8.561 & 107.8 & 45.26 & 56.03 & 173.5 & 8.564 & 107.8 \\
\hline I 221 & 59.68 & 45.03 & 171.0 & 8.805 & 118.7 & 59.88 & 45.23 & 171.0 & 8.802 & 118. \\
\hline A222 & 50.18 & 59.60 & 172.0 & 8.856 & 126.3 & 50.38 & 59.85 & 172.1 & 8.864 & 126. \\
\hline A 223 & 50.17 & - & 175.3 & 9.199 & 119.3 & 50.41 & - & 175.3 & 9.199 & 119 \\
\hline K224 & 56.08 & 50.32 & 177.0 & 8.759 & 122.6 & 56.30 & 50.46 & - & 8.757 & 122 \\
\hline
\end{tabular}




\begin{tabular}{|c|c|c|c|c|c|c|c|c|c|c|}
\hline Y225 & 55.22 & 56.06 & 174.2 & 9.853 & 129.0 & 55.37 & 56.27 & - & 9.848 & 129.0 \\
\hline Q226 & 54.39 & 55.26 & 173.5 & 9.010 & 131.6 & 54.58 & 55.44 & 173.7 & 8.999 & 131.6 \\
\hline I227 & 64.58 & 54.29 & 173.7 & 7.810 & 130.0 & 64.85 & 54.52 & 173.8 & 7.810 & 130.0 \\
\hline D228 & 52.65 & - & 175.7 & 8.426 & 118.4 & 52.87 & - & 175.6 & 8.426 & 118.5 \\
\hline D230 & - & - & - & - & - & 53.94 & - & 176.2 & 8.496 & 114.3 \\
\hline A231 & 51.60 & - & 174.9 & 7.665 & 124.3 & 51.85 & 53.99 & 174.9 & 7.667 & 124.2 \\
\hline $\mathrm{C} 232$ & 56.21 & 51.62 & 175.1 & 8.456 & 119.1 & 56.31 & 51.82 & 175.1 & 8.454 & 119.1 \\
\hline F233 & 55.63 & 55.98 & 171.8 & 9.235 & 128.0 & 55.81 & 56.44 & - & 9.233 & 128.0 \\
\hline S234 & 57.32 & 55.55 & - & 9.314 & 124.5 & 57.63 & 55.71 & - & 9.307 & 124.5 \\
\hline A235 & 50.65 & 57.38 & - & 8.022 & 122.9 & 50.92 & 57.40 & - & 8.018 & 122.8 \\
\hline K236 & 54.61 & 50.67 & 175.7 & 9.035 & 114.8 & 54.84 & 50.89 & 175.6 & 9.031 & 114.8 \\
\hline V237 & 59.49 & 54.62 & 174.6 & 8.948 & 117.0 & 59.78 & 54.83 & 174.1 & 8.941 & 117.0 \\
\hline N238 & - & - & - & - & - & 51.01 & 59.75 & 174.0 & 7.572 & 122.4 \\
\hline $\mathrm{N} 239$ & - & - & - & - & - & 55.17 & 51.00 & 176.5 & 8.471 & 113.6 \\
\hline S240 & - & - & - & - & - & 58.42 & 55.14 & - & 7.805 & 117.0 \\
\hline S241 & - & - & - & - & - & 59.82 & 58.46 & 172.8 & 8.139 & 109.6 \\
\hline L242 & - & - & - & - & - & 53.96 & 59.88 & 172.3 & 7.290 & 118.5 \\
\hline I 243 & 59.46 & 53.74 & 176.7 & 9.037 & 126.7 & 59.69 & 54.04 & 176.7 & 9.033 & 126.6 \\
\hline G244 & 44.03 & 59.50 & 174.8 & 9.281 & 114.6 & 44.25 & 59.73 & 174.8 & 9.280 & 114.6 \\
\hline L245 & 53.21 & 44.06 & 171.4 & 9.370 & 122.3 & 53.47 & 44.28 & 171.5 & 9.367 & 122.3 \\
\hline G246 & 44.67 & 53.20 & - & 9.177 & 110.6 & 44.85 & 53.48 & - & 9.172 & 110.5 \\
\hline Y247 & 56.17 & 44.66 & 170.9 & 9.087 & 125.5 & 56.42 & 44.88 & 171.0 & 9.082 & 125.4 \\
\hline T248 & 60.76 & 56.06 & 174.7 & 8.360 & 124.5 & 61.01 & 56.33 & 174.0 & 8.358 & 124.5 \\
\hline Q249 & 52.47 & 60.75 & - & 9.018 & 126.1 & 52.74 & 60.96 & - & 9.036 & 126.2 \\
\hline G254 & - & - & - & - & - & 47.22 & 62.84 & - & 8.564 & 110.9 \\
\hline I 255 & - & - & - & - & - & 60.19 & 47.35 & - & 8.233 & 121.7 \\
\hline K256 & - & - & - & - & - & 55.13 & - & 175.0 & 8.688 & 127.1 \\
\hline L257 & 52.98 & 54.83 & 174.7 & 8.805 & 127.8 & 53.15 & 55.02 & - & 8.795 & 127.8 \\
\hline T258 & 61.29 & 52.94 & 174.3 & 9.587 & 122.9 & 61.50 & 52.94 & 174.3 & 9.584 & 122.9 \\
\hline L259 & 52.87 & 61.36 & - & 9.223 & 129.5 & 53.07 & 61.42 & - & 9.228 & 129.6 \\
\hline S260 & 56.80 & 52.92 & 174.6 & 8.803 & 115.3 & 56.97 & 53.22 & - & 8.800 & 115.3 \\
\hline A261 & 51.12 & 56.73 & 172.8 & 9.544 & 120.6 & 51.31 & 57.03 & 172.8 & 9.536 & 120.6 \\
\hline L262 & 52.43 & 51.16 & 173.4 & 8.716 & 124.9 & 52.63 & 51.32 & 173.4 & 8.713 & 124.9 \\
\hline L263 & 52.31 & - & 175.2 & 9.489 & 126.2 & 52.54 & - & 175.2 & 9.486 & 126.2 \\
\hline D264 & 52.22 & - & 175.3 & 9.843 & 124.6 & 52.48 & - & 175.3 & 9.832 & 124.5 \\
\hline G265 & 46.02 & 52.28 & 176.9 & 8.458 & 112.4 & 46.24 & 52.53 & 176.8 & 8.455 & 112.4 \\
\hline K266 & 56.82 & 46.03 & 173.8 & 8.128 & 117.3 & 57.05 & 46.21 & 173.8 & 8.130 & 117.3 \\
\hline N267 & 51.77 & 56.79 & 177.1 & 7.425 & 117.5 & 52.00 & 57.03 & 177.1 & 7.424 & 117.5 \\
\hline V268 & 65.03 & 51.83 & 175.1 & 8.506 & 122.1 & 65.31 & 52.02 & 175.0 & 8.498 & 122.0 \\
\hline N269 & - & - & - & - & - & 54.53 & 65.25 & 177.4 & 8.304 & 116.9 \\
\hline $\mathrm{A} 270$ & 51.73 & 54.27 & 175.8 & 7.490 & 120.7 & 51.92 & 54.51 & 175.7 & 7.488 & 120.7 \\
\hline G271 & 44.74 & 51.71 & 177.7 & 7.692 & 106.7 & 44.95 & 51.92 & 177.7 & 7.686 & 106.7 \\
\hline G272 & 45.47 & 44.79 & 174.0 & 8.510 & 109.1 & 45.72 & 45.01 & 174.0 & 8.507 & 109.1 \\
\hline H273 & 54.54 & 45.47 & 174.0 & 7.246 & 120.7 & 54.77 & 45.71 & 173.9 & 7.244 & 120.7 \\
\hline K274 & 54.68 & 64.35 & 176.1 & 8.833 & 119.4 & 54.91 & 64.35 & 176.1 & 8.831 & 119.4 \\
\hline L275 & 53.53 & 54.74 & 175.2 & 8.654 & 121.6 & 53.75 & 54.98 & 175.2 & 8.647 & 121.6 \\
\hline G276 & 45.03 & 53.61 & 178.0 & 9.094 & 111.1 & 45.24 & 53.78 & 178.0 & 9.088 & 111.1 \\
\hline L277 & 53.79 & 45.09 & 170.9 & 8.202 & 121.0 & 54.03 & 45.37 & 171.0 & 8.207 & 121.0 \\
\hline G278 & 44.32 & 53.82 & - & 9.576 & 115.9 & 44.56 & 54.05 & - & 9.578 & 116.0 \\
\hline L279 & 53.10 & 55.27 & - & 9.046 & 130.0 & 53.54 & 44.48 & 171.7 & 8.699 & 125.0 \\
\hline E280 & 54.11 & 53.30 & 174.1 & 8.833 & 125.7 & 54.41 & 53.61 & - & 8.813 & 125.8 \\
\hline F281 & - & - & - & - & - & 54.37 & - & - & 9.037 & 126.1 \\
\hline
\end{tabular}




\begin{tabular}{rrrrrrrrrrr} 
A283 & 51.22 & 53.90 & 174.4 & 8.291 & 126.4 & 51.40 & 54.07 & 174.5 & 8.285 & 126.3 \\
R284 & 55.84 & 51.26 & - & 9.028 & 125.1 & - & - & - & - & - \\
S285 & 57.15 & 55.76 & 174.1 & 7.754 & 125.8 & 57.35 & 55.98 & 174.1 & 7.767 & 125.8 \\
\hline
\end{tabular}

Resonances printed in bold were obtained from E73V hVDAC1 spectra.

* Resonances of hVDAC1 that were shifted when expressed in the $p Q E 60$ vector (correct $N$-terminus, lacking RGS following the starting methionine)

Spectra were referenced automatically by the software nmrPipe. For the updated assignment, spectra were shifted additionally $+0.3 \mathrm{ppm}$ in the $13 \mathrm{C}$ dimension to match solid state shifts for residues T6-L10. 


\section{Resonance intensities}

Supporting table 2. Amide resonance intensities of $w t$, mutant and chemically modified hVDAC1 from ${ }^{1} \mathrm{H},{ }^{15} \mathrm{~N}$-TROSY spectra.

\begin{tabular}{|c|c|c|c|c|c|c|c|c|}
\hline residue & wt 1 & wt 2 & $\begin{array}{c}\text { wt } 3 \\
(\text { HetNOE) }\end{array}$ & E73V & DCCD & $\begin{array}{l}\text { ref for } \\
\text { DCCD }\end{array}$ & R15A & $\begin{array}{l}\text { ref for } \\
\text { R15A }\end{array}$ \\
\hline \multicolumn{9}{|l|}{ S1 } \\
\hline \multicolumn{9}{|l|}{ A2 } \\
\hline \multicolumn{9}{|l|}{ V3 } \\
\hline \multicolumn{9}{|l|}{ P4 } \\
\hline \multicolumn{9}{|l|}{ P5 } \\
\hline T6 & 440554 & 1062536 & 809337 & 1738277 & 1404814 & 1099390 & 9875169 & 13011617 \\
\hline Y7 & 381082 & 834578 & 723591 & 1115973 & 1261282 & 931198 & 8540341 & 11591049 \\
\hline A8 & 287496 & 649315 & 540021 & 1143023 & 1067881 & 752476 & 6846952 & 7782908 \\
\hline D9 & 225355 & 569926 & 287145 & 806626 & 867600 & 593940 & 5422194 & 4699444 \\
\hline L10 & 307865 & 603147 & 363170 & 1130182 & 906320 & 614325 & 4831600 & 8448662 \\
\hline \multicolumn{9}{|l|}{ G11 } \\
\hline K12 & 174221 & 377851 & 232932 & 572664 & 476265 & 304819 & 3393027 & 7851197 \\
\hline \multicolumn{9}{|l|}{$\mathrm{S} 13$} \\
\hline A14 & 184496 & 441270 & 427743 & 866829 & 647284 & 449619 & 4651987 & 5851031 \\
\hline $\mathrm{R} 15$ & 153734 & 335833 & 372844 & 633129 & 453572 & 352167 & & 5951308 \\
\hline D16 & & 401758 & 329262 & & 184170 & 151970 & & 2752737 \\
\hline V17 & 369099 & 559218 & & 914532 & 1315521 & 845156 & 3629806 & 5581760 \\
\hline \multicolumn{9}{|l|}{ F18 } \\
\hline \multicolumn{9}{|l|}{ T19 } \\
\hline K20 & 85411 & 124825 & & 1222868 & 400298 & 385483 & 6377096 & 2993506 \\
\hline \multicolumn{9}{|l|}{ G21 } \\
\hline Y22 & 174132 & 465146 & 200140 & 804457 & 405488 & 340790 & 3874113 & 5461914 \\
\hline \multicolumn{9}{|l|}{ G23 } \\
\hline $\mathrm{F} 24$ & 141548 & 431972 & & 742420 & 352489 & 221584 & -865899 & 3624802 \\
\hline G25 & 179373 & 206280 & 278022 & 450543 & 408221 & 283777 & 2849454 & 3300637 \\
\hline L26 & 258324 & 452338 & 431267 & 791405 & 858936 & 565793 & 4685190 & 7663420 \\
\hline $\mathrm{I} 27$ & 221565 & 481966 & 613556 & 749055 & 1622460 & 757758 & 7177612 & 8332091 \\
\hline K28 & 334062 & 687841 & 651862 & 1599236 & 1552401 & 970224 & 7452879 & 8172292 \\
\hline L29 & 283254 & 453370 & 497483 & 1169966 & 1262176 & 652370 & 7425362 & 7951448 \\
\hline D30 & 346980 & 537316 & 496114 & 1526643 & & & 9754228 & 15006444 \\
\hline L31 & 274194 & 483141 & 481968 & 1446083 & 650082 & 580355 & 6848410 & 7993619 \\
\hline K32 & 225670 & 315685 & & 954167 & & & & \\
\hline $\mathrm{T} 33$ & 259528 & 485743 & 387146 & 1168204 & 603379 & 421211 & 4296500 & 7225816 \\
\hline K34 & 111488 & 250203 & 194892 & 1039898 & 513587 & 500491 & 6405678 & 9316959 \\
\hline S35 & 299013 & 455311 & 512489 & 1554063 & 495766 & 349161 & 5800332 & 8777135 \\
\hline \multicolumn{9}{|l|}{ E36 } \\
\hline \multicolumn{9}{|l|}{ N37 } \\
\hline G38 & 277767 & 495818 & 572617 & 1632900 & 221386 & 238247 & 4070260 & 7110327 \\
\hline L39 & & & & 1464435 & 345866 & & & \\
\hline E40 & 151972 & & 304492 & 1023795 & & & & 5206450 \\
\hline F41 & & & & 1204228 & & & & 8974974 \\
\hline $\mathrm{T} 42$ & 119121 & 224121 & 292924 & 880166 & 195656 & 199393 & 3163920 & 4282732 \\
\hline
\end{tabular}




\begin{tabular}{|c|c|c|c|c|c|c|c|c|}
\hline S43 & 126670 & 233402 & 180534 & 749011 & 324916 & 258927 & 2950652 & 3517588 \\
\hline S44 & 213757 & 387337 & 351794 & 857320 & & & 5662102 & 6228296 \\
\hline G45 & 239660 & 485408 & 406501 & & 1027811 & 542656 & 4619160 & 5065164 \\
\hline S46 & 302124 & 640296 & 477950 & 1185368 & 1087901 & 638974 & 6277059 & 7412852 \\
\hline A47 & & & & 997652 & 937793 & 590794 & 7555244 & 10887460 \\
\hline N48 & 346189 & 584831 & 412217 & 1208634 & 1022195 & 703568 & 8380852 & 9212798 \\
\hline \multicolumn{9}{|l|}{ T49 } \\
\hline \multicolumn{9}{|l|}{ E50 } \\
\hline \multicolumn{9}{|l|}{$\mathrm{T} 51$} \\
\hline \multicolumn{9}{|l|}{ T52 } \\
\hline K53 & 369099 & 713961 & & 1224439 & 1315521 & 845156 & 14273739 & 10430291 \\
\hline V54 & 513780 & 1042606 & 1034533 & 1598396 & 1459487 & 945016 & 11836006 & 12913888 \\
\hline T55 & 484980 & 942909 & 654838 & 1739087 & 1661313 & 1121336 & 10058095 & 11691546 \\
\hline G56 & 280939 & 399811 & 284552 & 1444811 & 818326 & 406898 & 5444347 & 5947614 \\
\hline S57 & 159809 & 288421 & 275015 & 746031 & 501135 & 259440 & 2854788 & 3402296 \\
\hline L58 & 174938 & 279120 & & 1101772 & 823529 & 417051 & 3939204 & 5249304 \\
\hline E59 & 178522 & 424101 & 432717 & 1600989 & 1027663 & 524535 & 5721912 & 6684620 \\
\hline T60 & 147076 & 493983 & & 1390147 & 705530 & 318346 & 5698946 & 4715052 \\
\hline K61 & 247745 & 406354 & 400213 & 1123919 & 703604 & 515669 & 5649488 & 5900526 \\
\hline Y62 & 104219 & 129774 & 269453 & 1346323 & 355447 & 229262 & 1397553 & 2974974 \\
\hline R63 & & 715888 & 227447 & 2086570 & 856626 & 19000 & & \\
\hline W64 & & & & 1381324 & 221593 & 19000 & & \\
\hline \multicolumn{9}{|l|}{ T65 } \\
\hline \multicolumn{9}{|l|}{ E66 } \\
\hline Y67 & 123535 & 108063 & 258877 & 1128462 & 640731 & 176632 & 1820413 & 1769048 \\
\hline G68 & 248362 & 381968 & 390132 & 968937 & 644606 & 413546 & 4365126 & 4731158 \\
\hline L69 & 381315 & 779684 & 604687 & 1751916 & 838688 & 828608 & 5463652 & 8815891 \\
\hline $\mathrm{T} 70$ & & & & 1192504 & 1308966 & 894990 & 5801670 & 6299332 \\
\hline F71 & 152573 & 302035 & 271255 & 1440416 & 536080 & 388740 & 4818384 & 4849651 \\
\hline $\mathrm{T} 72$ & & & & 1393763 & 804922 & 19000 & & 2622466 \\
\hline E73 & & & & 1824434 & & & & 6192545 \\
\hline K74 & & & & 1522199 & 326633 & 19000 & & \\
\hline W75 & & & & 540961 & & & & \\
\hline N76 & 172291 & 1106606 & 561938 & 945494 & 582352 & 151160 & 3584966 & 4457636 \\
\hline $\mathrm{T} 77$ & & & & 891229 & 814286 & 388357 & 3407180 & 4268710 \\
\hline D78 & 157719 & 281785 & 276569 & 813135 & 653710 & 396660 & 3298981 & 4485787 \\
\hline N79 & 206545 & 362648 & 296509 & 1021146 & 651003 & 383077 & 3340246 & 3865701 \\
\hline $\mathrm{T} 80$ & 193272 & 281904 & 216183 & 882569 & 433167 & 271565 & 4341648 & 3380704 \\
\hline L81 & 255223 & 410308 & 377035 & 1154264 & 829393 & 453220 & 4066926 & 6108502 \\
\hline G82 & & & & 1310868 & 636160 & 19000 & & \\
\hline $\mathrm{T} 83$ & & & & 1283635 & 962962 & 19000 & & \\
\hline E84 & & & & 1107224 & & & & \\
\hline I85 & 121378 & 328042 & 269569 & 929742 & 510101 & 348064 & 2706072 & 3166158 \\
\hline T86 & 367234 & 891924 & 614636 & 1354610 & 1083740 & 1015890 & 9361609 & 9494709 \\
\hline V87 & 395264 & 736292 & 575515 & 1613616 & & & 10560856 & 9306908 \\
\hline E88 & & & & 1357826 & 615049 & 19000 & & \\
\hline D89 & 280454 & 632217 & 500004 & 1003929 & 973545 & 737195 & 11419224 & 14882085 \\
\hline Q90 & 173321 & 385416 & 265677 & 1333791 & 883697 & 501647 & 4502844 & 5440726 \\
\hline L91 & & & & 1243838 & 904332 & 177319 & & \\
\hline A92 & & & & 746197 & 667164 & 174811 & & \\
\hline R93 & 139174 & 486211 & 361010 & 1056147 & 751324 & 518998 & 3416653 & 5680790 \\
\hline G94 & 258084 & 487913 & 436446 & 993771 & 836098 & 541403 & 4354113 & 6029896 \\
\hline
\end{tabular}




\begin{tabular}{|c|c|c|c|c|c|c|c|c|}
\hline L95 & 366848 & 765458 & 541701 & 1266906 & 1281061 & 816352 & 7996836 & 9124619 \\
\hline K96 & 192866 & 301655 & 324780 & 873326 & 591469 & 379362 & 4071108 & 5594112 \\
\hline L97 & 329670 & 761657 & & 1599236 & & & 5145794 & 10568192 \\
\hline T98 & 298424 & 721479 & 581292 & 1167772 & 985002 & 682782 & 5495254 & 6706422 \\
\hline F99 & 226438 & 428862 & 436592 & 1158078 & 742260 & 560190 & 3253288 & 5589234 \\
\hline D100 & 182674 & 342536 & 234531 & 1143508 & 867367 & 411820 & 3042787 & 4840170 \\
\hline S101 & 430064 & 911290 & 762825 & 1697492 & 930241 & 1159634 & 8139737 & 10135203 \\
\hline S102 & 264378 & 610211 & 611350 & 1697492 & 1211398 & 802552 & 4803022 & 6562466 \\
\hline F103 & 154862 & 406665 & 392463 & 1435166 & 770982 & 514299 & 7716306 & 3842882 \\
\hline S104 & 218728 & 573599 & 569736 & 2124504 & 1366361 & 859106 & 2676060 & 6590363 \\
\hline \multicolumn{9}{|l|}{ P105 } \\
\hline \multicolumn{9}{|l|}{ N106 } \\
\hline \multicolumn{9}{|l|}{ T107 } \\
\hline G108 & 183703 & 484668 & 357241 & 893512 & 443888 & 442709 & 2326016 & 3378613 \\
\hline K109 & 478109 & 1298335 & 740229 & 2466127 & 1559991 & 1402022 & 7411290 & 8510469 \\
\hline K110 & 670840 & 1791634 & 1245295 & 2826966 & 1976119 & 1612457 & 7446411 & 16212565 \\
\hline N111 & 463945 & 1084735 & 797440 & 2045444 & 815499 & 1243798 & 5575672 & 10819433 \\
\hline A112 & 326760 & 1066566 & 912318 & 1383848 & 859277 & 1446944 & 9675279 & 10652085 \\
\hline K113 & 171405 & 586253 & 402847 & 1369446 & 1196932 & 675147 & 2899645 & 4699440 \\
\hline I114 & 316737 & 630781 & 567150 & 1586029 & 1333692 & 757971 & 4183227 & 6216447 \\
\hline K115 & 327170 & 664544 & 523062 & 1136467 & 896553 & 627307 & 6189038 & 7079495 \\
\hline T116 & 319779 & 803401 & 529300 & 1257146 & 1054878 & 761463 & 6337523 & 7084870 \\
\hline G117 & 353372 & 678058 & 555598 & 1225574 & 964504 & 665993 & 8153372 & 7645923 \\
\hline Y118 & 388624 & 763990 & 730161 & 1378598 & 1109249 & 665998 & 4992425 & 10887460 \\
\hline K119 & 405268 & 776056 & 662241 & 1317466 & 1128462 & 941770 & 5434068 & 9585249 \\
\hline R120 & 315470 & 759741 & 725592 & 1247404 & 1012382 & 780954 & 11419224 & 14882085 \\
\hline E121 & 554989 & 1124150 & 948763 & 1575165 & 1308966 & 894990 & 8267314 & 11010265 \\
\hline H122 & 574472 & 892485 & 866190 & 1659785 & 1466185 & 1221758 & 6649401 & 8334836 \\
\hline I123 & 520620 & 802353 & 759974 & 1446040 & 1182765 & 899723 & 8755018 & 9689382 \\
\hline N124 & 497450 & 921857 & 753291 & 1514444 & 1871781 & 1173264 & 12695649 & 12171224 \\
\hline L125 & 483016 & 798603 & 830289 & 1361310 & 1075427 & 833817 & 8130122 & 8981915 \\
\hline G126 & 296327 & 545782 & 455123 & 931196 & 782046 & 590796 & 5810376 & 5542772 \\
\hline $\mathrm{C} 127$ & 361069 & 844980 & 635738 & 1212660 & 1091938 & 992646 & 8383263 & 8083986 \\
\hline D128 & 329186 & 748502 & 544678 & 970322 & 842904 & 646276 & 7396912 & 7434520 \\
\hline M129 & 251233 & 592351 & 426706 & 1279973 & 958560 & 607836 & 1984226 & 4935860 \\
\hline D130 & 472364 & 1029505 & 788593 & 1229804 & 1367388 & 1446944 & 9675279 & 12817438 \\
\hline F131 & 454786 & 857752 & 872089 & 1342105 & 1446515 & 1266357 & 2203945 & 3485899 \\
\hline D132 & 167569 & 308293 & 428643 & 2336347 & 2443255 & 744214 & 3942175 & 3321736 \\
\hline I133 & & & & 1970377 & & & 3423716 & 4547944 \\
\hline A134 & 689855 & 1708013 & 1722172 & 2600852 & 2426422 & 1771093 & 12400270 & 16223238 \\
\hline G135 & 1061907 & 2043336 & 1953358 & 2898368 & 2776846 & 2263098 & 14933121 & 22233692 \\
\hline \multicolumn{9}{|l|}{ P136 } \\
\hline S137 & 316195 & 617998 & 730777 & 1359574 & 1055504 & 622217 & 5262343 & 7063153 \\
\hline I138 & 390698 & 845173 & 802766 & & 1129190 & 931986 & 6702340 & 9556620 \\
\hline R139 & 346777 & 760826 & 704181 & 1194068 & 1067407 & 794002 & 6522704 & 7182536 \\
\hline G140 & 318401 & 641152 & 605069 & 1080010 & 1115546 & 776636 & 6803141 & 6741333 \\
\hline A141 & 446024 & 987807 & 767268 & 1358969 & 1519588 & 1049790 & 10016355 & 9373384 \\
\hline L142 & 362150 & 714961 & 697840 & 1152553 & 1011560 & 720868 & 7109608 & 7568504 \\
\hline V143 & 888253 & 1808460 & 1604180 & 2546806 & 1864502 & 1671898 & 12762997 & 19045788 \\
\hline L144 & 502152 & 961291 & 777637 & 1343264 & 1313596 & 981177 & 8816937 & 8224043 \\
\hline G145 & 581299 & 913540 & 848834 & 1503747 & 1277673 & 1011544 & 9134614 & 9966833 \\
\hline Y146 & 588983 & 1095025 & 802965 & 1685949 & 1592224 & 1180734 & 10455336 & 15006444 \\
\hline
\end{tabular}




\begin{tabular}{|c|c|c|c|c|c|c|c|c|}
\hline E147 & 589780 & 1235687 & 946299 & 1847372 & 1468120 & 1115675 & 2524452 & \\
\hline G148 & 497291 & 648226 & 692350 & 1226563 & 1065937 & 744151 & 3874255 & 6840134 \\
\hline W149 & 291336 & 554207 & 463787 & 1112735 & 1169492 & 990036 & 5934094 & 7603702 \\
\hline L150 & 399051 & 814376 & 739703 & 1251421 & 1271016 & 902110 & 7097966 & 8505891 \\
\hline A151 & 524004 & 864073 & 784462 & 1589809 & 1715965 & 1247605 & 9946441 & 9387423 \\
\hline G152 & 383329 & 710003 & 611793 & 1044851 & 1126751 & 827039 & 6693502 & 7233840 \\
\hline Y153 & 416335 & 756951 & 583037 & 1062896 & 1051775 & 771204 & 7426438 & 7126306 \\
\hline Q154 & 552801 & 1030648 & 704189 & 1439546 & 1294849 & 1005548 & 10286579 & 10318753 \\
\hline M155 & 460011 & 804764 & 839673 & 1129732 & 1267521 & 977749 & 8961292 & 9026513 \\
\hline N156 & 445060 & 818484 & 957866 & 1287391 & 1356084 & 1162564 & 9322469 & 11637273 \\
\hline F157 & 380305 & 568239 & 699959 & 1054693 & 813673 & 632463 & 4570082 & 8025832 \\
\hline E158 & 529857 & 955340 & 811527 & 1405560 & 1390842 & 1058120 & 7727374 & 10981006 \\
\hline T159 & 359924 & 715496 & 630942 & 1174162 & 1224107 & 772076 & 5774602 & 7986038 \\
\hline A160 & 341940 & 741141 & 753304 & 817637 & 1145096 & 522049 & 6539283 & 7209513 \\
\hline \multicolumn{9}{|l|}{ K161 } \\
\hline S162 & 451022 & 823583 & 645096 & 1168317 & 1154865 & 827428 & 6456671 & 8262966 \\
\hline R163 & 306918 & 559995 & 414046 & 591664 & 717750 & 614779 & 5222290 & 6084068 \\
\hline V164 & 504912 & 838226 & 789725 & 934544 & 989266 & 951297 & 12843779 & 13308589 \\
\hline T165 & 342969 & 672721 & 646926 & 1252138 & 1209895 & 816220 & 7715812 & 7295818 \\
\hline Q166 & 192771 & 300943 & 403469 & 420392 & 522307 & 390032 & 3997318 & 4528854 \\
\hline S167 & 698774 & 1243976 & 1154384 & 1832027 & 1700934 & 1350030 & 14462942 & 14817835 \\
\hline N168 & 354315 & 623885 & 444698 & 955170 & 743845 & 590252 & 7973741 & 7674900 \\
\hline F169 & 528000 & 953299 & 825707 & 2655463 & 1607866 & 1034943 & 9326240 & 10628997 \\
\hline A170 & 431658 & 829462 & 762386 & 1243229 & 1219358 & 1015857 & 8445309 & 9160651 \\
\hline V171 & 403053 & 869981 & 735432 & 1433674 & 1096759 & 826595 & 6013320 & 8651576 \\
\hline G172 & 454518 & 872758 & 615414 & 1434537 & 1153270 & 896513 & 7419708 & 8492355 \\
\hline Y173 & 515042 & 994560 & 799587 & 1569974 & & & 9681342 & 10991474 \\
\hline K174 & 492571 & 851073 & 890161 & 1352525 & 1026965 & 836221 & 5847948 & 12743942 \\
\hline T175 & 294819 & 719989 & 600269 & 973764 & 814364 & 561355 & & 10051243 \\
\hline \multicolumn{9}{|l|}{ D176 } \\
\hline \multicolumn{9}{|l|}{ E177 } \\
\hline F178 & 298145 & 678360 & 545057 & 958365 & 1068694 & 765633 & 14273739 & 7954566 \\
\hline Q179 & 322092 & 627666 & 425348 & 1059431 & 943414 & 706122 & 5901333 & 8166736 \\
\hline L180 & 235315 & 532133 & 540595 & 807809 & 488043 & 390331 & 4818384 & 6802186 \\
\hline H181 & 136895 & 336402 & 335545 & 624866 & & 167659 & 3127603 & 3628951 \\
\hline \multicolumn{9}{|l|}{ T182 } \\
\hline N183 & 330724 & 686125 & 483605 & 950459 & 589972 & 419864 & 4098430 & 7297338 \\
\hline V184 & 452439 & 663639 & 647981 & 1375923 & 961027 & 707805 & 7088076 & 7874204 \\
\hline N185 & 441770 & 727686 & 637085 & 911088 & 724846 & 747066 & 7922791 & 9066257 \\
\hline D186 & 888253 & 1808460 & 1604180 & 2503500 & 1864502 & 1671898 & 13436111 & 19045788 \\
\hline G187 & 464985 & 677516 & 788188 & 1247600 & 953966 & 753369 & 8210264 & 9475110 \\
\hline T188 & 294282 & 421335 & 475367 & 768915 & 615079 & 418667 & 4745341 & 5133176 \\
\hline E189 & 326509 & 582808 & 610740 & 935220 & 785565 & 624569 & 9329218 & 7289830 \\
\hline F190 & 546188 & 1060561 & 805356 & 1332828 & 1673057 & 1215408 & 11226459 & 13310636 \\
\hline G191 & 503677 & 810632 & 689056 & 1372629 & 1109782 & 870863 & 8929836 & 9099788 \\
\hline G192 & 532288 & 849801 & 769806 & 1452618 & 1153906 & 922844 & 9119252 & 9550837 \\
\hline S193 & 225556 & 363421 & 269124 & 656526 & 298688 & 258008 & 2698967 & 5440014 \\
\hline I194 & 412856 & 762172 & 676865 & 1214102 & 930241 & 1159634 & 12655046 & 9525287 \\
\hline Y195 & 378663 & 798276 & 547577 & 1214492 & 978355 & 742818 & 6940227 & 9164488 \\
\hline Q196 & 558368 & 1046374 & 708286 & 1629380 & 1221139 & 996798 & 9738020 & 13386658 \\
\hline K197 & 408250 & 697339 & 637826 & 1316005 & 960874 & 747844 & 9330301 & 12043493 \\
\hline V198 & 234514 & 250487 & 326402 & 773973 & 394359 & 319141 & 5285914 & 6088577 \\
\hline
\end{tabular}




\begin{tabular}{|c|c|c|c|c|c|c|c|c|}
\hline N199 & 65936 & 142899 & 270662 & 390195 & 154433 & 155211 & 2516024 & 3048826 \\
\hline \multicolumn{9}{|l|}{ K200 } \\
\hline \multicolumn{9}{|l|}{ K201 } \\
\hline \multicolumn{9}{|l|}{ L202 } \\
\hline E203 & 323725 & & & 964872 & 635393 & 513701 & 5392417 & 5725432 \\
\hline T204 & 360104 & 384237 & 629731 & 1147936 & 556935 & 441848 & 6826400 & 7181590 \\
\hline A205 & 355506 & 596986 & 586142 & 1146853 & 707094 & 605898 & 6935904 & 8765713 \\
\hline V206 & 828847 & 1122567 & 1192305 & 2364576 & 1690284 & 1185038 & 9187394 & 14866412 \\
\hline N207 & 407508 & 762712 & 670178 & 992876 & 865660 & 773449 & 7716306 & 8345912 \\
\hline L208 & 453671 & 722126 & 634437 & 1395266 & 968096 & 705753 & 9271527 & 9273021 \\
\hline A209 & 398182 & 704561 & 694615 & 1299460 & 928656 & 673381 & 8270999 & 9389998 \\
\hline W210 & 394962 & 645110 & 578808 & 1228038 & 974163 & 710836 & 7690348 & 8816423 \\
\hline T211 & 521857 & 745637 & 800269 & 1364073 & 1023178 & 799146 & 9300452 & 12446687 \\
\hline A212 & 830044 & 1438590 & 1473591 & 2001303 & 1907595 & 1890510 & 15447201 & 18847974 \\
\hline G213 & 256848 & 651963 & 802773 & 1253314 & 454671 & 449061 & 2849404 & 6262118 \\
\hline N214 & 455131 & 1064360 & 1087136 & 2059034 & 923170 & 876438 & 10317799 & 12977206 \\
\hline \multicolumn{9}{|l|}{ S215 } \\
\hline N216 & 509648 & 1325911 & 1384467 & 2395821 & 892332 & 861064 & 6638580 & 12469431 \\
\hline T217 & 627992 & 1282427 & 1408233 & 2132751 & 1118466 & 1020120 & 9472953 & 14540212 \\
\hline R218 & 804406 & 1140769 & 1349156 & 1867991 & 1532684 & 1302746 & 22881980 & 25440368 \\
\hline F219 & 660213 & 1025790 & 1151177 & 1898350 & 1368202 & 1069946 & 13019668 & 15834107 \\
\hline G220 & 346228 & 537486 & 481954 & 1034242 & 770614 & 557859 & 5743523 & 6497346 \\
\hline I221 & 423247 & 833981 & 890503 & 1419395 & 1141852 & 706180 & 8595036 & 10143531 \\
\hline A222 & 375347 & 607209 & 507466 & 1201171 & 870780 & 755369 & 9631223 & 8131970 \\
\hline A223 & 348146 & 670413 & 434476 & 1075773 & 703505 & 545139 & 6215808 & 7037902 \\
\hline K224 & 273958 & 322671 & 312506 & 1022756 & 338407 & 269238 & 5688706 & 6939826 \\
\hline Y225 & 200657 & 6205 & 273955 & 731032 & & & 3780255 & 5244519 \\
\hline Q226 & 288178 & 128685 & 270862 & 912437 & 275527 & 258590 & 6272038 & 8401501 \\
\hline I227 & 222610 & 125187 & & 666693 & 212697 & 205798 & 3403704 & 5287010 \\
\hline D228 & 156265 & 192475 & 365305 & 539725 & 290305 & 263853 & 3725430 & 4570635 \\
\hline \multicolumn{9}{|l|}{ P229 } \\
\hline D230 & 184919 & 242517 & 316772 & 622992 & 379224 & 324468 & 3981024 & 4398134 \\
\hline A231 & 194256 & 185036 & 172825 & 759781 & 429724 & 353401 & 2962124 & 4481807 \\
\hline C232 & 199666 & 401758 & 329399 & 855342 & & & 3747107 & 6151559 \\
\hline $\mathrm{F} 233$ & 210508 & 206992 & 364978 & 819986 & 152427 & 267602 & 4845919 & 5805264 \\
\hline S234 & & & & 733900 & & & 10560856 & 9306908 \\
\hline A235 & 259733 & 357096 & 443695 & 1138033 & 692946 & 474555 & 5180796 & 7459442 \\
\hline K236 & 321736 & 520342 & 382586 & 959503 & 622087 & 469409 & 4878690 & 6803488 \\
\hline V237 & 828847 & 1122567 & 1192305 & 2364576 & 1690284 & 1185038 & 8815223 & 14866412 \\
\hline N238 & 703652 & 1106606 & 764421 & 1438750 & 1250830 & 1027582 & 12540827 & 17835962 \\
\hline N239 & 193292 & 363829 & 342288 & 720034 & 511732 & 300914 & 3755180 & 5142406 \\
\hline S240 & 227924 & 439588 & 464090 & 1068429 & 745933 & 887366 & 4328512 & 5139662 \\
\hline S241 & 319686 & 465016 & 493454 & 1420958 & 685985 & 507529 & 5487416 & 5232626 \\
\hline L242 & 363955 & 491441 & 421857 & 1073773 & 844933 & 587213 & 5691216 & 7569917 \\
\hline I 243 & 494277 & 912782 & 773796 & 1331166 & 1190799 & 1001465 & 11039488 & 10559032 \\
\hline G244 & 427740 & 620964 & 422538 & 1124986 & 870983 & 634464 & 6368939 & 6984348 \\
\hline L245 & 360514 & 521275 & 301632 & 1069974 & 748892 & 564786 & 5062510 & 6965145 \\
\hline G246 & 152446 & 216777 & 262457 & 597825 & 299541 & 153833 & 3261138 & 3841642 \\
\hline Y247 & 260021 & 228736 & 340827 & 667609 & 350738 & 334936 & 5921522 & 7153580 \\
\hline T248 & 259832 & 561251 & 509130 & 849920 & & & 4329538 & 6172394 \\
\hline Q249 & 200196 & 629373 & 381596 & 846604 & & & 5692040 & 5909284 \\
\hline
\end{tabular}


L251

K252

P253

\begin{tabular}{|c|c|c|c|c|c|c|c|c|}
\hline G254 & 141779 & 326195 & 498406 & 515224 & 366682 & 298084 & 2043460 & 3972598 \\
\hline I255 & 156291 & 362589 & & 874668 & 614802 & 567903 & 4077251 & 5478091 \\
\hline K256 & 193242 & 242344 & 534521 & 991645 & & & 4474643 & 5513008 \\
\hline L257 & 233863 & 282955 & 482540 & 765335 & 521363 & 407087 & 5321252 & 7127464 \\
\hline $\mathrm{T} 258$ & 187368 & 258149 & 310099 & 691054 & 477585 & 356903 & 3830378 & 4037518 \\
\hline L259 & 179655 & 375273 & 228068 & 664625 & 372649 & 271856 & 3808186 & 4609527 \\
\hline S260 & 185195 & 333918 & 261694 & 647301 & 425895 & 296135 & 3405566 & 3974200 \\
\hline A261 & 230095 & 304523 & 269803 & 698885 & 472121 & 333462 & 2853184 & 4081986 \\
\hline L262 & 546890 & 1009747 & 1029766 & 1822198 & 1653136 & 1199254 & 9956239 & 15444304 \\
\hline L263 & 434726 & 549205 & 503429 & 1182230 & 790970 & 589772 & 6124640 & 8412161 \\
\hline D264 & 219265 & 211409 & & 585476 & 436846 & 311346 & 3569892 & 5974358 \\
\hline G265 & 603220 & 918425 & 808706 & 1769635 & 1481941 & 1087805 & 10635518 & 11404512 \\
\hline K266 & 614638 & 930592 & 898437 & 1478494 & 1257131 & 966501 & 9101977 & 11866212 \\
\hline N267 & 647477 & 1082793 & 958406 & 1883121 & 1615799 & 1184304 & 10952782 & 11809505 \\
\hline V268 & 779231 & 1851710 & 1800154 & 2613774 & 2201638 & 1741375 & 13515461 & 18102552 \\
\hline N269 & 427427 & 1024865 & 955560 & 1535975 & 876172 & 754420 & 5965364 & 9005541 \\
\hline A270 & 692709 & 1738990 & 1553192 & 2202122 & 1559991 & 1545966 & 9953046 & 16628584 \\
\hline G271 & 884005 & 1329090 & 1569230 & 2252438 & 2017261 & 1782779 & 12362752 & 18340280 \\
\hline G272 & 1027715 & 1887776 & 2004314 & 2967816 & 2110325 & 1998461 & 14803656 & 23400148 \\
\hline $\mathrm{H} 273$ & 454814 & 520507 & 566262 & 1238187 & 803310 & 730539 & 7173579 & 9948912 \\
\hline K274 & 426488 & 594244 & 630142 & 1224382 & 1206729 & 867302 & 12655046 & 9676580 \\
\hline L275 & 508341 & 1000012 & 759141 & 1506494 & 1515157 & 1284061 & 8866678 & 12296697 \\
\hline G276 & 331730 & 578617 & 329670 & 1392152 & 898838 & 563569 & 4974094 & 6705072 \\
\hline L277 & 614284 & 1164190 & 1100105 & 2356222 & 1836200 & 1415004 & 13711392 & 14975284 \\
\hline G278 & 215864 & 372005 & 419532 & 836356 & 597451 & 358668 & 3289095 & 4142948 \\
\hline L279 & 382267 & 553039 & 485570 & 1300920 & 991613 & 914191 & 6771918 & 8968395 \\
\hline E280 & 270004 & 510466 & 266750 & 779093 & 1259543 & 850045 & 7881411 & 9612863 \\
\hline F281 & 200196 & 629373 & & 1119865 & & & & \\
\hline \multicolumn{9}{|l|}{ Q282 } \\
\hline A283 & 243019 & 520987 & 627825 & 1528890 & 882707 & 524719 & 7312128 & 8892941 \\
\hline \multicolumn{9}{|l|}{ R284 } \\
\hline S285 & & & & 2846818 & 4208397 & 4167714 & 27390710 & 38854556 \\
\hline
\end{tabular}




\section{E RDC measurements}

\section{E.1 Estimation of accuracy of the "RDC-TROSY" experiment for} measurements of ${ }^{1} \mathrm{H}-{ }^{15} \mathrm{~N}$ residual dipolar couplings

In order to extract ${ }^{1} \mathrm{D}_{\mathrm{NH}}$ values for hVDAC1 weakly aligned in charged polyacrylamide gels, we tested the scaled $\left({ }^{1} \mathrm{~J}_{\mathrm{NH}}+{ }^{1} \mathrm{D}_{\mathrm{NH}}\right) 2 \mathrm{D}$ "RDC-TROSY" (Bhattacharya et al. 2010) experiment. For this purpose, the experiment was tested on an isotropic sample of $0.5 \mathrm{mM} w t$ hVDAC1 (in 3\% LDAO, $25 \mathrm{mM}$ Bis-Tris $\mathrm{pH} 6.8,5 \% \mathrm{D}_{2} \mathrm{O}$ ) that allows extraction of ${ }^{1} \mathrm{~J}_{\mathrm{NH}}$ values. $\kappa$ values of $0,0.25,0.5,0.75$, and 1.0 were used and the spectra were recorded in an interleaved fashion. While the spectrum with the lowest $\kappa$ value was of good quality, with increasing $\kappa$ values resonances became significantly broad and distorted (Supporting figure $4 \mathrm{~A}$ ). ${ }^{1} \mathrm{~J}_{\mathrm{NH}}$ couplings were extracted by linear fitting to varieties of combinations of the five spectra. The lowest variation in ${ }^{1} \mathrm{~J}_{\mathrm{NH}}$ values was achieved from the three spectra of $\kappa=0,0.5$, and 0.75 (Supporting figure 4B). This suggests that a minimum of $\kappa$ is required, but that at a certain threashold value extraction of couplings loses accuracy. Nevertheless, variations in ${ }^{1} \mathbf{J}_{\mathrm{NH}}$ were approximately twice as large as the variations for ${ }^{1} \mathbf{J}_{\mathrm{NH}}$ values extracted from interleaved TROSY/HSQC spectra of equal signal-to-noise (Supporting figure 4B). Thus, in case of hVDAC1 the interleaved TROSY/HSQC spectra proved superior for exctration of ${ }^{1} \mathrm{D}_{\mathrm{NH}}$ values. 
A
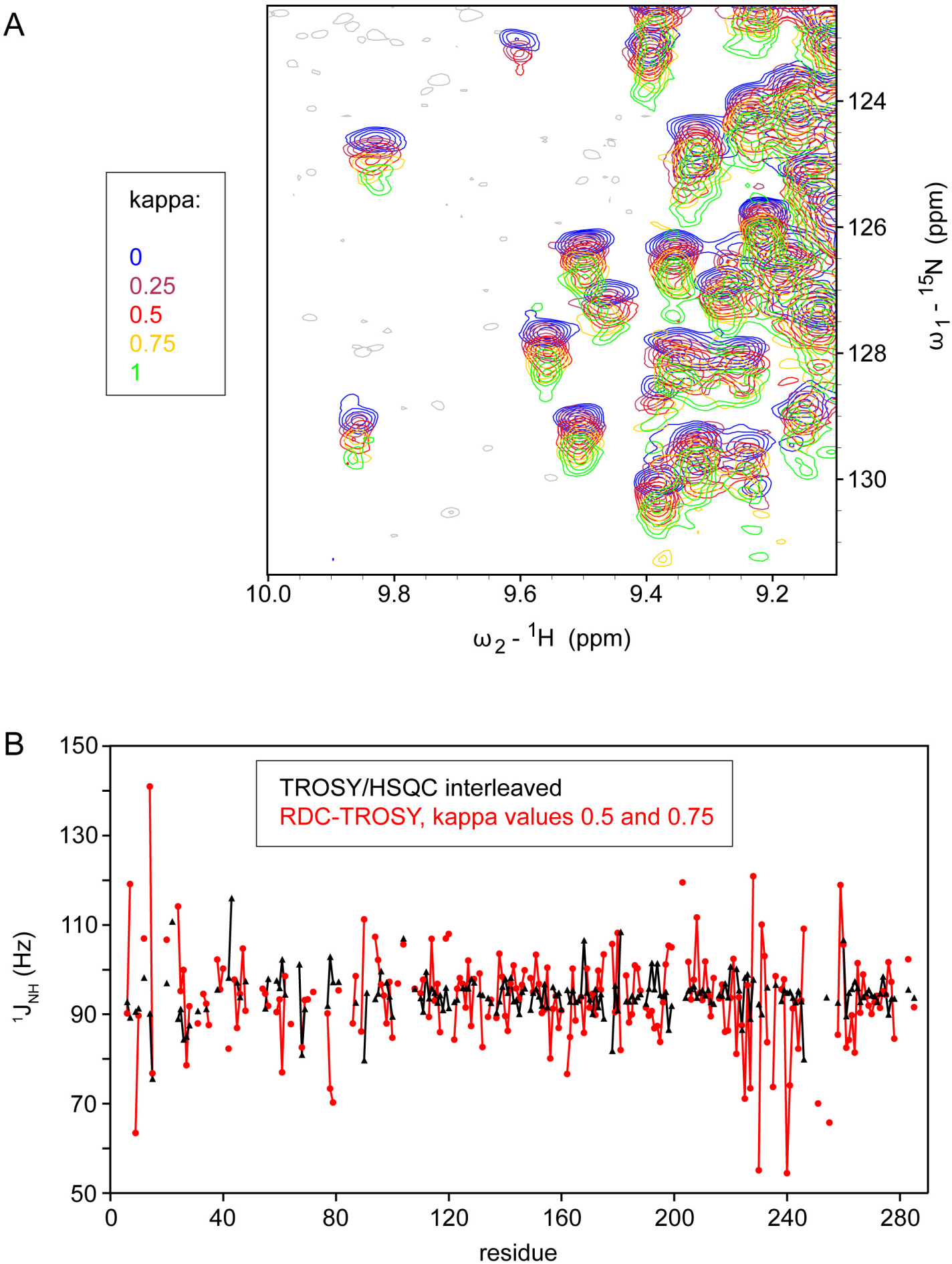

Supporting figure 4. Estimation of the accuracy of 2D RDC-TROSY experiments. (A) Overlay of spectral regions from the interleaved RDC-TROSY spectra, measured with indicated values of $\kappa$. (B) Comparison of ${ }^{1} \mathrm{~J}_{\mathrm{NH}}$ couplings exctracted from the RDC-TROSY and from an interleaved TROSY/HSQC of isotropic $w t \mathrm{hVDAC} 1$. 


\section{E.2 RDC values}

Supporting table 3. ${ }^{1} \mathrm{D}_{\mathrm{NH}}$ values for C232S hVDAC1.

\begin{tabular}{|c|c|c|c|c|c|c|c|}
\hline $\begin{array}{l}\text { Residue } \\
\text { number }\end{array}$ & $\begin{array}{l}\text { Residue } \\
\text { type }\end{array}$ & ${ }^{1} \mathrm{D}_{\mathrm{NH}}$ obs* & ${ }^{1} \mathrm{D}_{\mathrm{NH}} \mathrm{calc}^{\#}$ & $\begin{array}{l}\text { Residue } \\
\text { number }\end{array}$ & $\begin{array}{l}\text { Residue } \\
\text { type }\end{array}$ & ${ }^{1} \mathrm{D}_{\mathrm{NH}}$ obs* & ${ }^{1} \mathrm{D}_{\mathrm{NH}} \mathrm{calc}^{\#}$ \\
\hline 6 & THR & -9.56 & 1.2301 & 151 & ALA & -3.06 & 0.3073 \\
\hline 9 & ASP & -0.74 & -6.9955 & 152 & GLY & -5.64 & -4.3649 \\
\hline 14 & ALA & -6.98 & -6.5521 & 154 & GLN & -14.28 & 0.9658 \\
\hline 15 & ARG & -5.30 & -5.5451 & 158 & GLU & -15.14 & -4.6585 \\
\hline 25 & GLY & -13.50 & -3.7581 & 164 & VAL & -9.08 & -2.6557 \\
\hline 26 & LEU & 9.66 & -0.8671 & 171 & VAL & -6.12 & -1.0840 \\
\hline 45 & GLY & -2.10 & 0.1890 & 172 & GLY & 4.86 & -4.1788 \\
\hline 48 & ASN & -24.32 & -6.7475 & 179 & GLN & -34.48 & -5.9350 \\
\hline 81 & LEU & 8.42 & 6.1356 & 184 & VAL & 8.28 & -3.2451 \\
\hline 85 & ILE & 14.52 & 9.8904 & 192 & GLY & -5.88 & -4.0622 \\
\hline 86 & THR & 12.30 & 4.6642 & 196 & GLN & -3.50 & 0.1216 \\
\hline 94 & GLY & -11.84 & -4.2444 & 210 & TRP & -5.60 & -6.0233 \\
\hline 100 & ASP & 4.60 & 3.2209 & 219 & PHE & 7.16 & -1.3651 \\
\hline 109 & LYS & -13.76 & -8.3993 & 220 & GLY & -6.58 & -1.7458 \\
\hline 110 & LYS & 7.76 & 0.6054 & 223 & ALA & 3.18 & 0.3877 \\
\hline 111 & ASN & -5.80 & 2.5952 & 226 & GLN & -16.06 & -3.8225 \\
\hline 112 & ALA & 7.06 & 8.3533 & 227 & VAL & 5.58 & 8.6016 \\
\hline 113 & LYS & 13.76 & 4.7086 & 230 & ASP & -22.24 & -10.0476 \\
\hline 115 & LYS & -2.46 & 2.4054 & 236 & LYS & 1.14 & -1.0296 \\
\hline 116 & THR & 20.68 & 7.4617 & 239 & ASN & -12.66 & -8.2225 \\
\hline 117 & GLY & -5.12 & -0.6860 & 243 & ILE & 2.86 & 3.0477 \\
\hline 119 & LYS & -9.86 & -1.2379 & 254 & GLY & -8.36 & -7.3547 \\
\hline 122 & HIS & -10.22 & -3.5392 & 263 & LEU & -1.74 & 1.4538 \\
\hline 126 & GLY & 9.02 & 1.5349 & 264 & ASP & -20.14 & -3.9822 \\
\hline 129 & VAL & 7.88 & 9.3533 & 265 & GLY & -12.14 & -3.5972 \\
\hline 132 & ASP & -9.54 & -6.5688 & 266 & LYS & -4.62 & 16.7725 \\
\hline 134 & ALA & -6.08 & -6.4314 & 267 & ASN & 8.90 & -8.0003 \\
\hline 137 & SER & 7.26 & 2.3267 & 268 & VAL & -14.08 & -10.5444 \\
\hline 139 & ARG & -5.80 & -0.8058 & 269 & ASN & -15.96 & 2.6941 \\
\hline 140 & ALA & 20.22 & 10.5844 & 271 & GLY & 0.72 & -3.7243 \\
\hline 141 & LEU & 0.08 & -0.9442 & 272 & GLY & -14.08 & 17.0050 \\
\hline 144 & LEU & 11.42 & 8.6285 & 273 & HIS & -5.48 & -3.9244 \\
\hline 145 & GLY & -11.90 & -6.4430 & 275 & LEU & 5.20 & 5.6805 \\
\hline 146 & TYR & -1.62 & -2.8046 & 277 & LEU & -6.30 & 0.6762 \\
\hline 147 & GLU & -5.84 & -4.7312 & 283 & ALA & 8.84 & 1.3919 \\
\hline
\end{tabular}

${ }^{1} D_{N H}$ values determined from interleaved TROSY/HSQC spectra measured on ${ }^{2} \mathrm{H} /{ }^{15} \mathrm{~N} C 232 S \mathrm{hVDACl}$.

\# Back-calculated ${ }^{1} D_{N H}$ values using the crystal structure of mVDAC1 (PDB code: $\left.3 E M N\right)$ and the program PALES. 


\section{F Pulse programs and acquisition parameters}

In the following the pulse programs and acquisition parameters for the following experiments on Bruker instruments are given:

- RDC-TROSY (Bhattacharya et al. 2010) for measurement of ${ }^{1} \mathbf{J}_{\mathrm{NH}}$ values on isotropic $w t \mathrm{hVDAC1}$.

- TROSY-based Hahn-echo experiment (Wang et al. 2003) for measurement of chemical exchange rates contributing to ${ }^{15} \mathrm{~N}$ transverse relaxation. Acquisition parameters are given for $w t \mathrm{hVDAC1}$.

\section{F.1 Pulse program of RDC-TROSY}

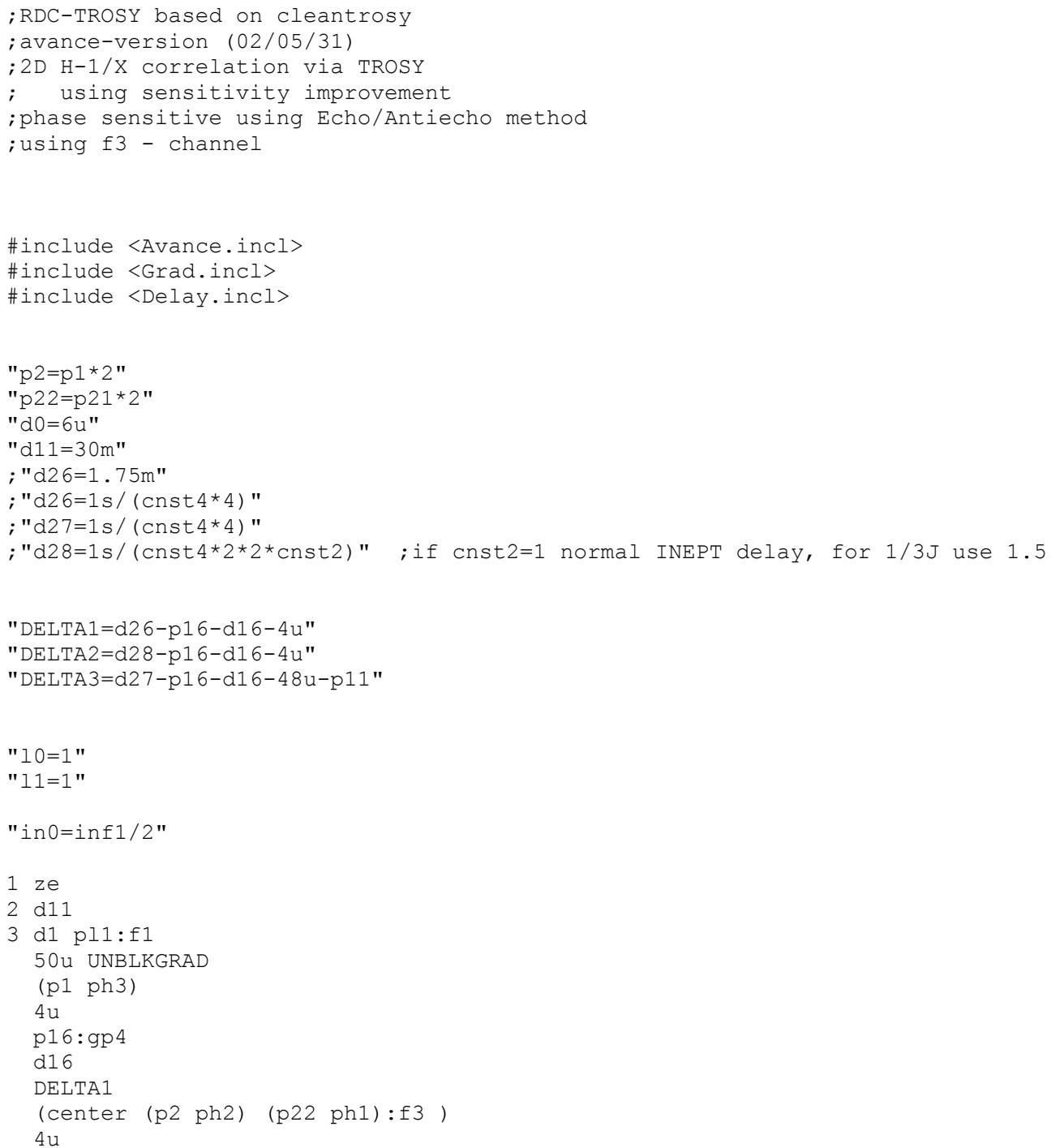




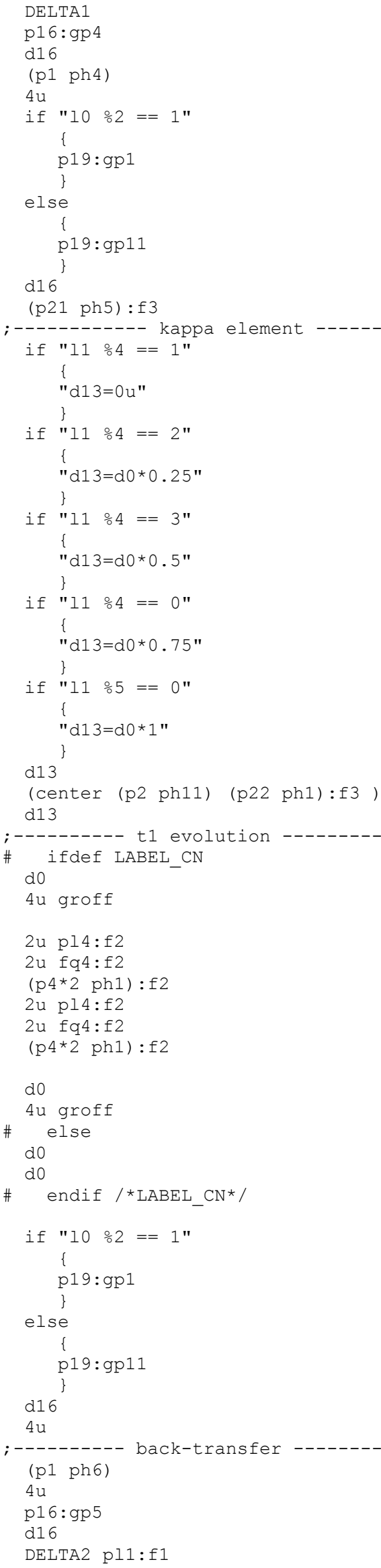




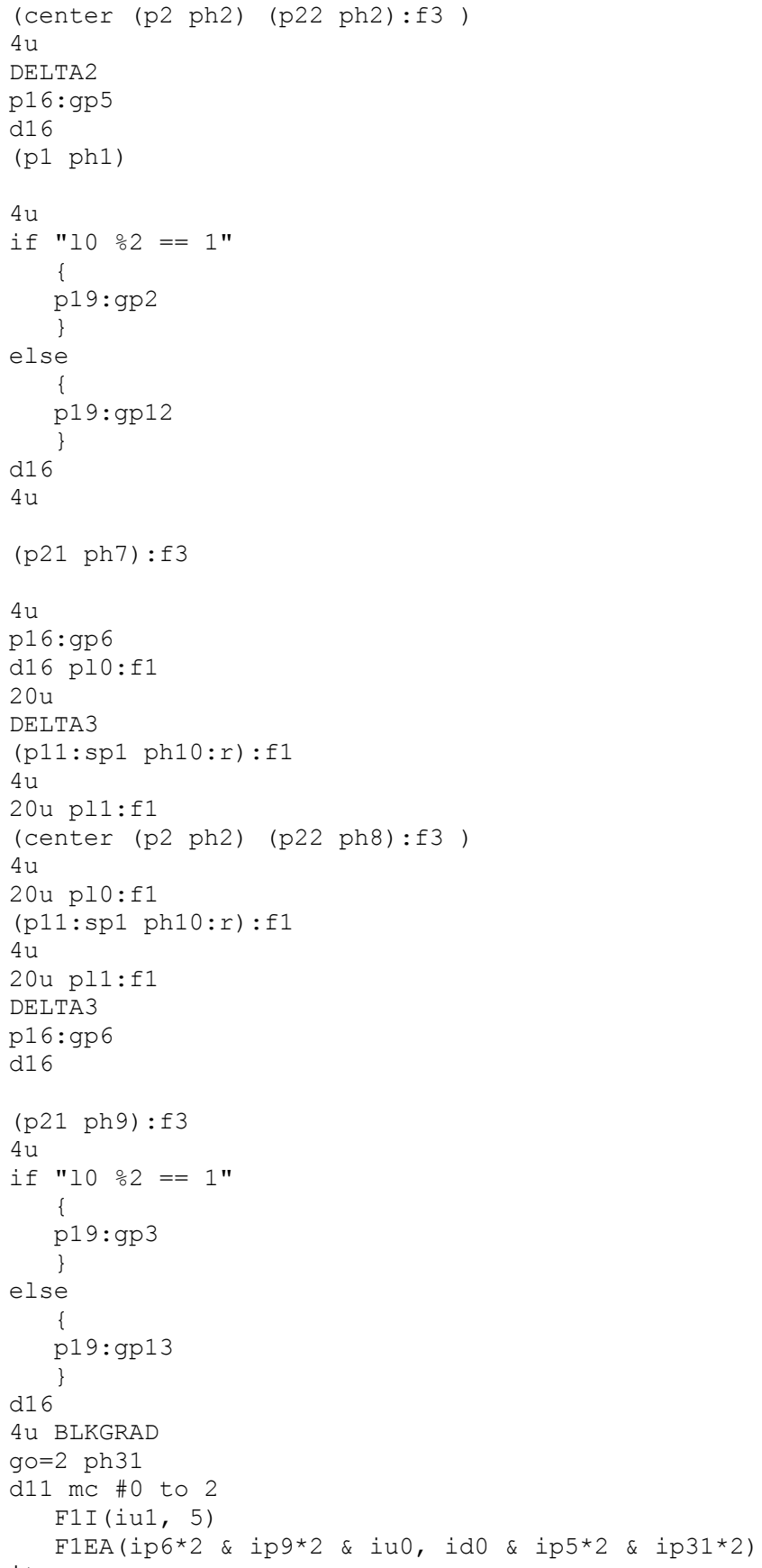




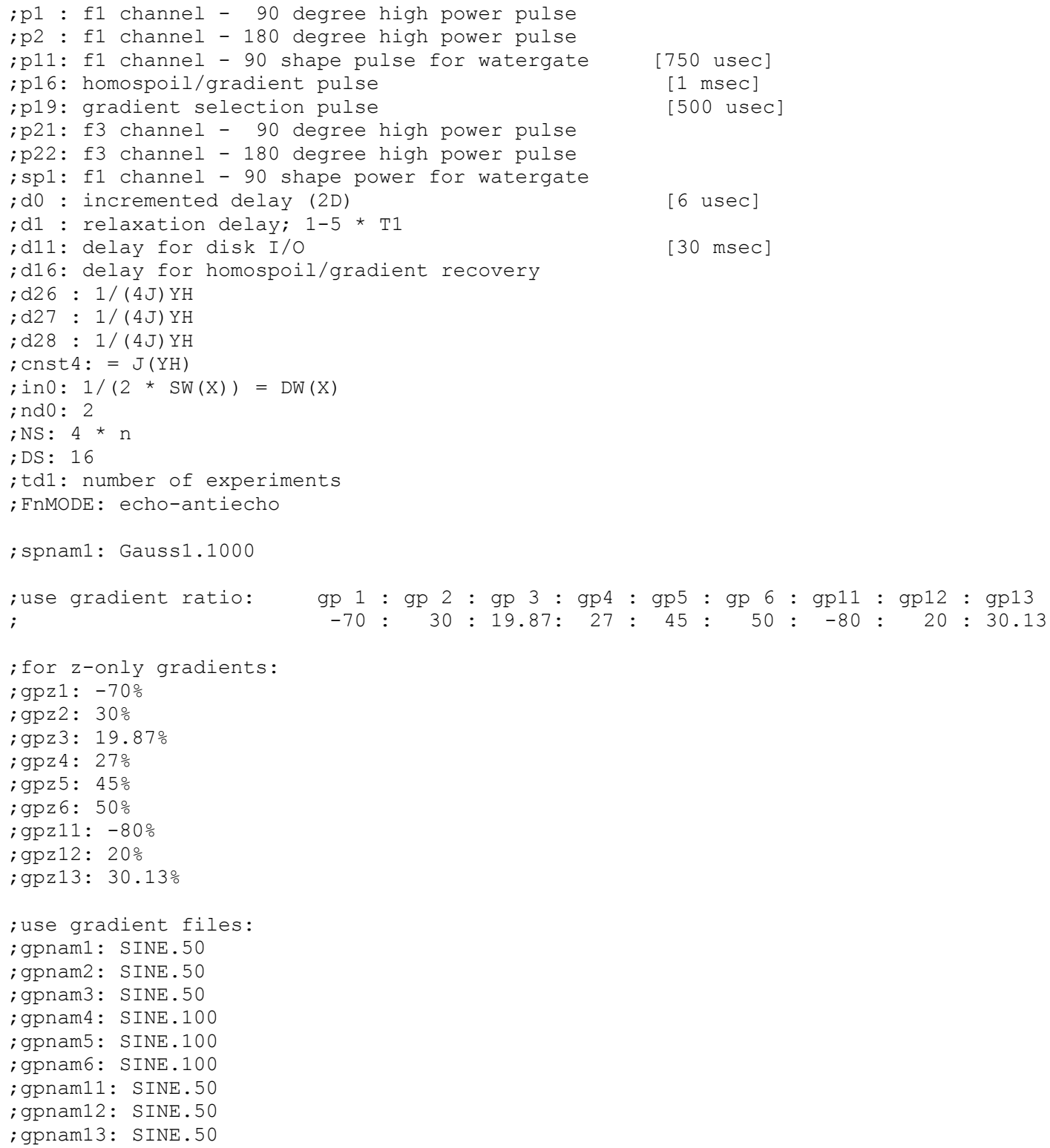

[750 usec]

$[1 \mathrm{msec}]$
$[500$ usec

[6 usec]

[30 $\mathrm{msec}]$ 


\section{F.2 Acquisition parameters for RDC-TROSY measured on an isotropic sample of wt hVDAC1}

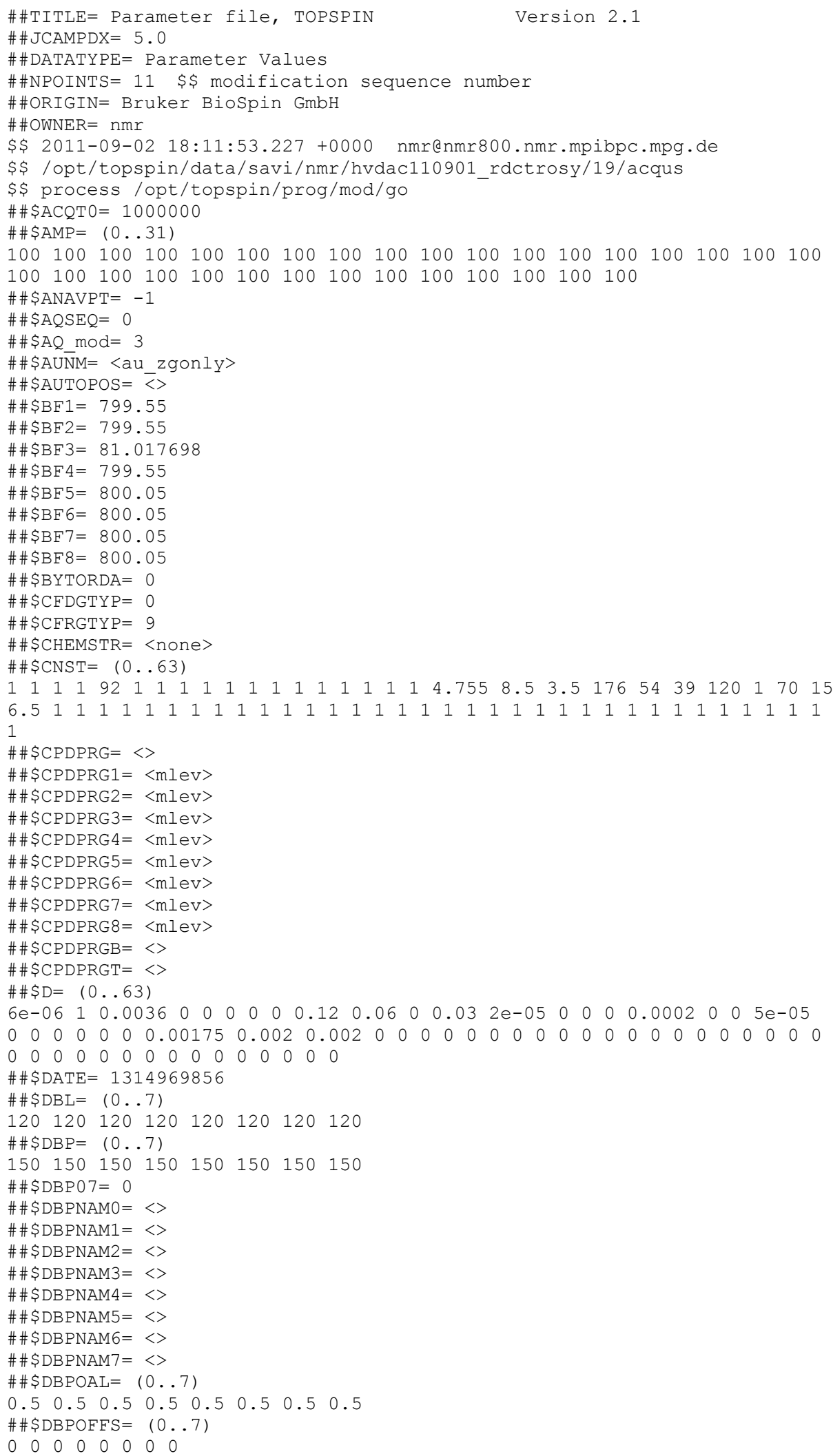


$\# \# \$ D E=6.5$

$\# \#$ \#ECBNUC $=\langle\mathrm{Off}>$

$\# \#$ \#ECIM $=1560$

$\# \# \$ D E C N U C=\langle O f f\rangle$

$\# \#$ \#ECSTAT $=4$

$\# \#$ DIGMOD $=1$

\#\#\$DIGTYP $=12$

$\# \# \mathrm{DL}=(0 \ldots 7)$

$\begin{array}{llllllll}10 & 120 & 120 & 120 & 120 & 120 & 120 & 120\end{array}$

$\# \#$ \#P $=(0 \ldots 7)$

$\begin{array}{lllllllll}150 & 150 & 150 & 150 & 150 & 150 & 150 & 150\end{array}$

$\# \#$ \#P $07=0$

$\# \# \$$ DPNAME $0=<>$

\#\#\$DPNAME $1=<>$

\#\# \$DPNAME $2=<>$

\#\# \$DPNAME $3=<>$

\#\# \$DPNAME $4=<>$

\#\#\$DPNAME $5=<>$

\#\#\$DPNAME $6=<>$

\#\#\$DPNAME $7=<>$

$\# \#$ \$DPOAL $=(0.7)$

$\begin{array}{llllllll}0.5 & 0.5 & 0.5 & 0.5 & 0.5 & 0.5 & 0.5 & 0.5\end{array}$

$\# \# \$ D P O F F S=(0.7)$

$\begin{array}{lllllllll}0 & 0 & 0 & 0 & 0 & 0 & 0 & 0\end{array}$

$\# \#$ \#QDMODE $=0$

$\# \# \$ D R=22$

$\# \#$ \#S $=16$

\#\# \$DSLIST $=<$ SSSSSSSSSSSSSS $>$

\#\# \$DSPFIRM $=0$

$\# \#$ DSPFVS $=20$

$\# \#$ \#TYPA $=0$

$\# \# \$ E X P=<>$

\#\#\$F1LIST $=\langle 111111111111111>$

\#\# $\$$ F2LIST $=\langle 222222222222222\rangle$

\#\#\$F3LIST $=\langle 333333333333333\rangle$

\#\# $\$$ FCUCHAN $=(0 \ldots 9)$

$0 \begin{array}{llllllllll}0 & 2 & 0 & 1 & 0 & 0 & 0 & 0 & 0 & 0\end{array}$

\#\#\$FL1 $=90$

$\# \#$ \# $\mathrm{FL} 2=90$

\#\#\$FL3= 90

$\# \# \$ F L 4=90$

$\# \# \$ F O V=20$

\#\#\$FQ1LIST $=\langle$ freqlist $>$

\#\#\$FQ2LIST $=\langle$ freqlist $>$

$\# \#$ \#EQ3LIST $=\langle$ freqlist $>$

\#\#\$FQ4LIST $=\langle$ Caco.savi $>$

\#\#\$FQ5LIST $=\langle$ freqlist $>$

\#\#\$FQ6LIST $=<$ freqlist $>$

$\# \#$ \#EQ7LIST $=\langle$ freqlist>

\#\# $\$ F Q 8 \mathrm{LIST}=\langle$ freqlist $>$

\#\#\$FRQLO3= 1247019.85000001

$\# \#$ \#RQLO3N=0

$\# \#$ \# $=(0 \ldots 7)$

$\begin{array}{llllllll}83 & 83 & 83 & 83 & 83 & 83 & 83 & 83\end{array}$

$\# \#$ \#FTLPGN $=0$

$\# \# \$ F W=125000$

$\# \#$ \#nMODE $=0$

$\# \#$ \# $\mathrm{ETYPE}=0$

\#\#\$GP031=0

\#\# $\$$ GPNAM $0=\langle$ sine.100 0

$\# \#$ \$GPNAM1 $=\langle$ SINE. 50 $\rangle$

\#\# \$GPNAM10 $=\langle$ sine. 100$\rangle$

\#\#\$GPNAM1 $1=\langle$ SINE. 50 $\rangle$

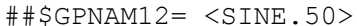

\#\# \$GPNAM1 $3=\langle$ SINE. 50 $\rangle$

\#\# \$GPNAM1 $4=\langle$ sine. 100$\rangle$

\#\#\$GPNAM15 $=\langle$ sine. 100$\rangle$

\#\#\$GPNAM1 $6=\langle$ sine. 100$\rangle$

\#\#\$GPNAM1 $7=\langle$ sine. 100$\rangle$

\#\#\$GPNAM1 $8=\langle$ sine. 100$\rangle$

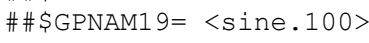

\#\#\$GPNAM $2=\langle$ SINE. 50$\rangle$

$\# \#$ \$GPNAM2 $0=\langle$ sine.100 $\rangle$

\#\# \$GPNAM21 $=\langle$ sine. 100$\rangle$ 


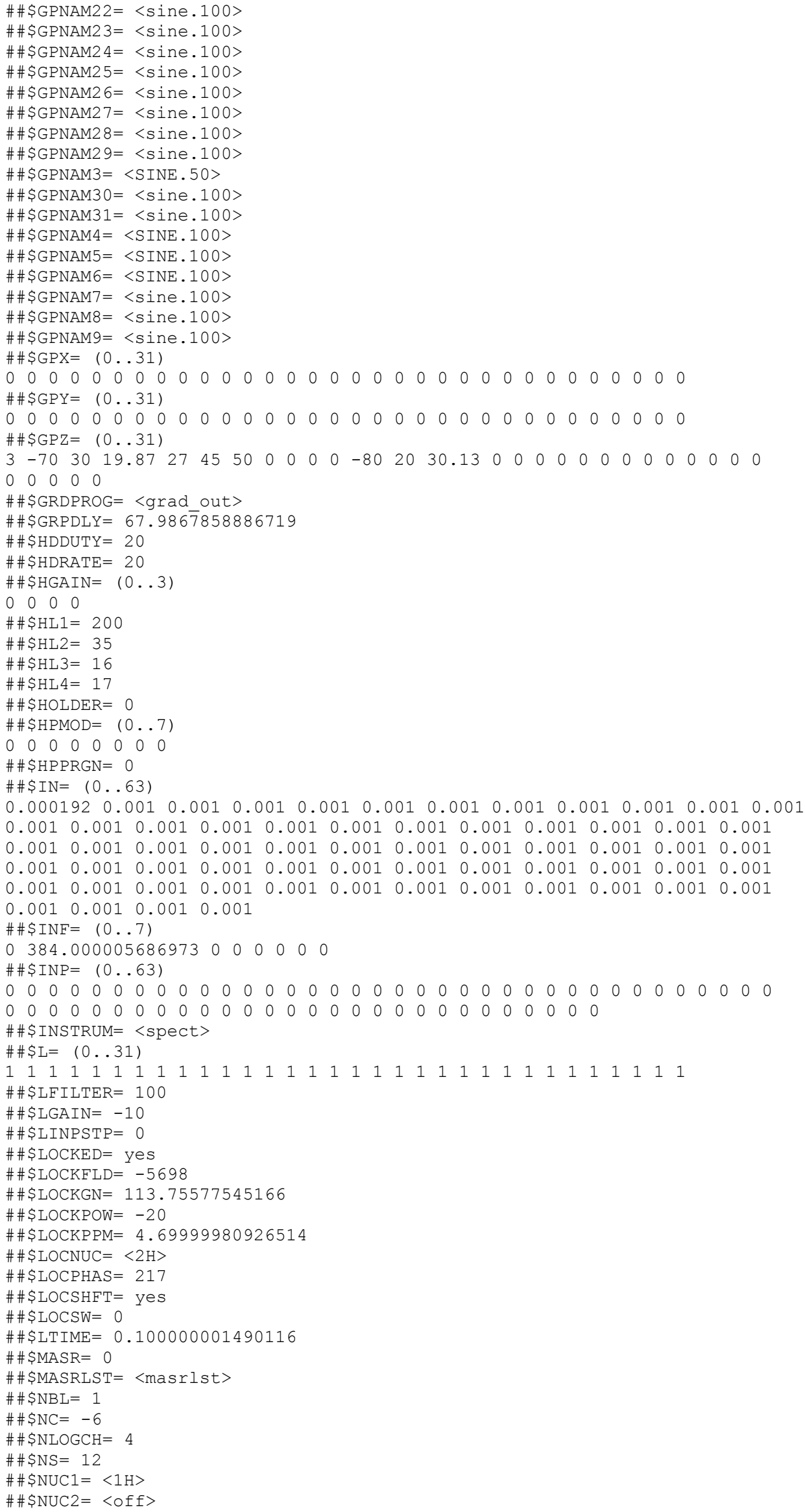




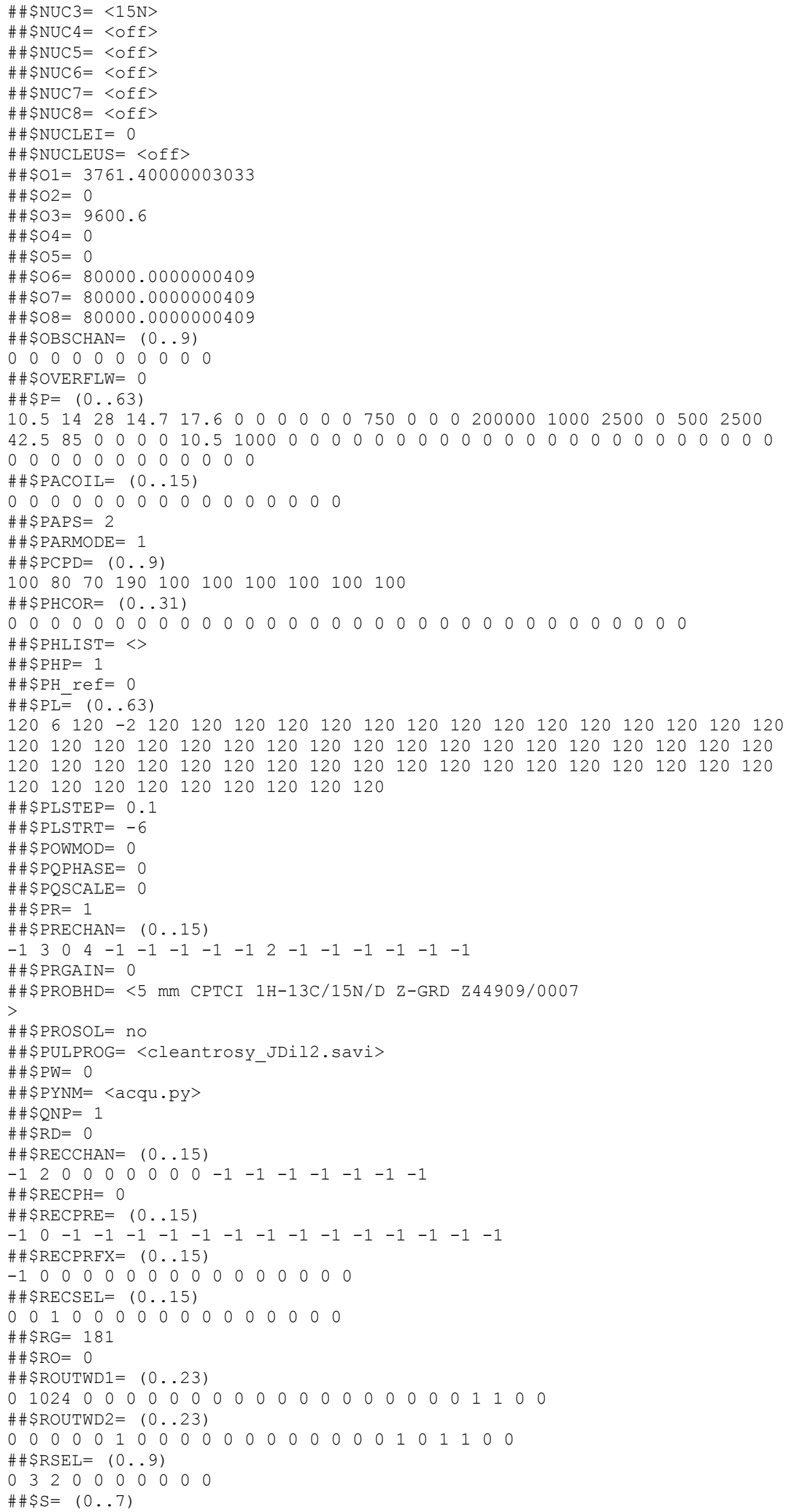




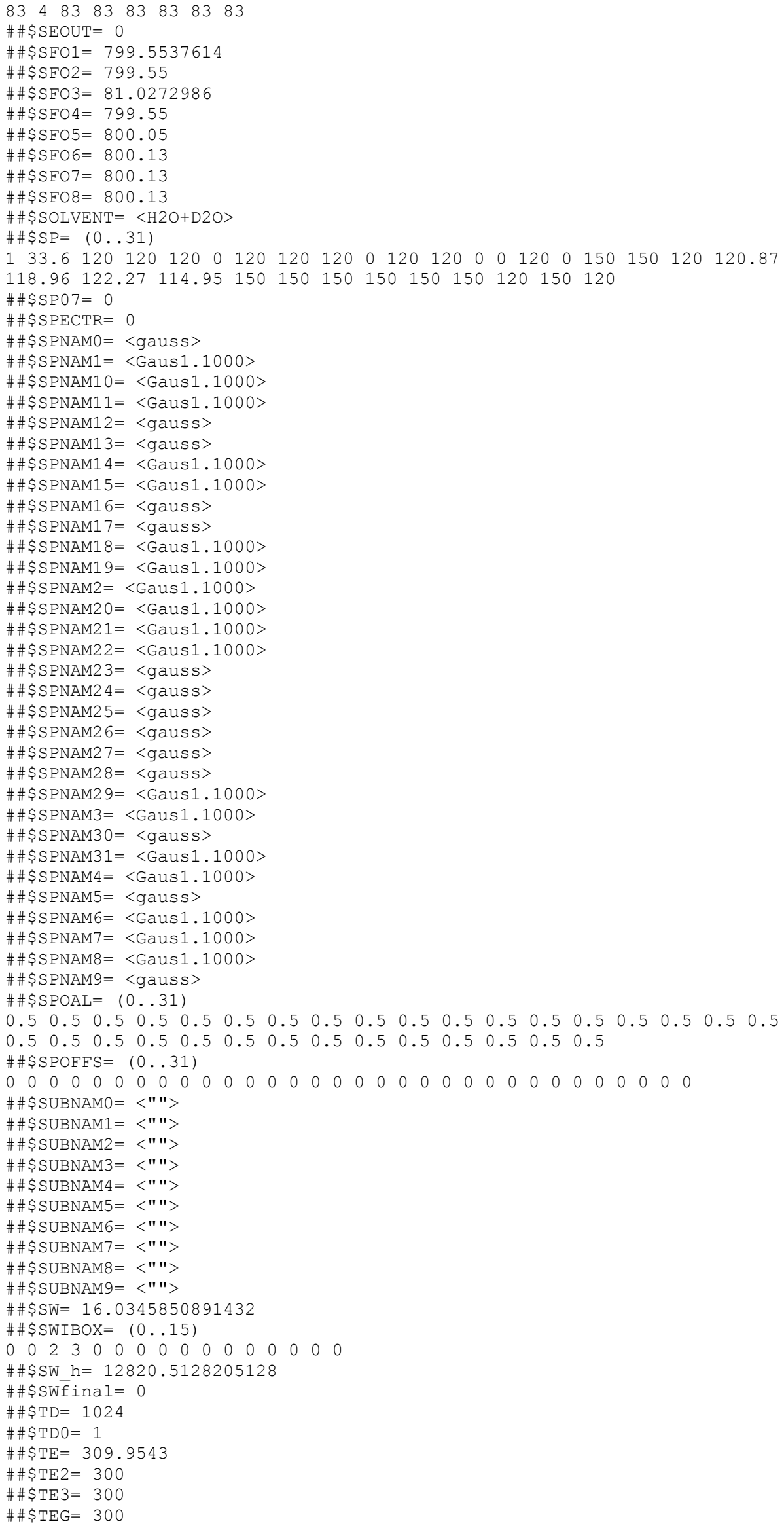




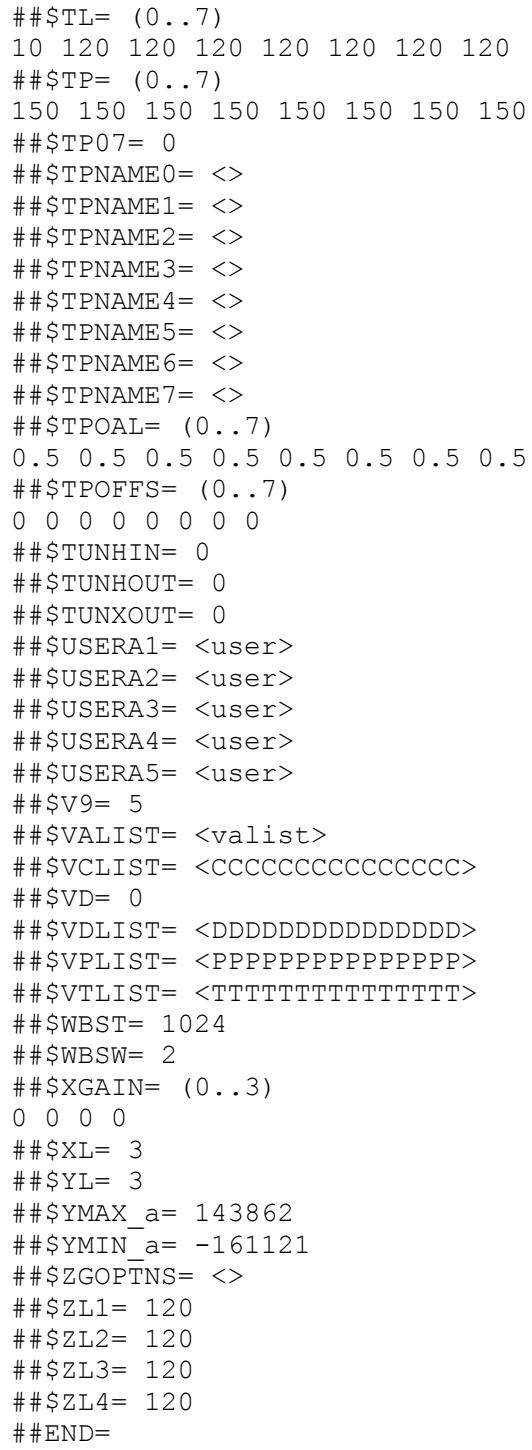




\section{F.3 Pulse program for measurement of chemical exchange rates contributing}

\section{to ${ }^{15} \mathrm{~N}$ transverse relaxation}

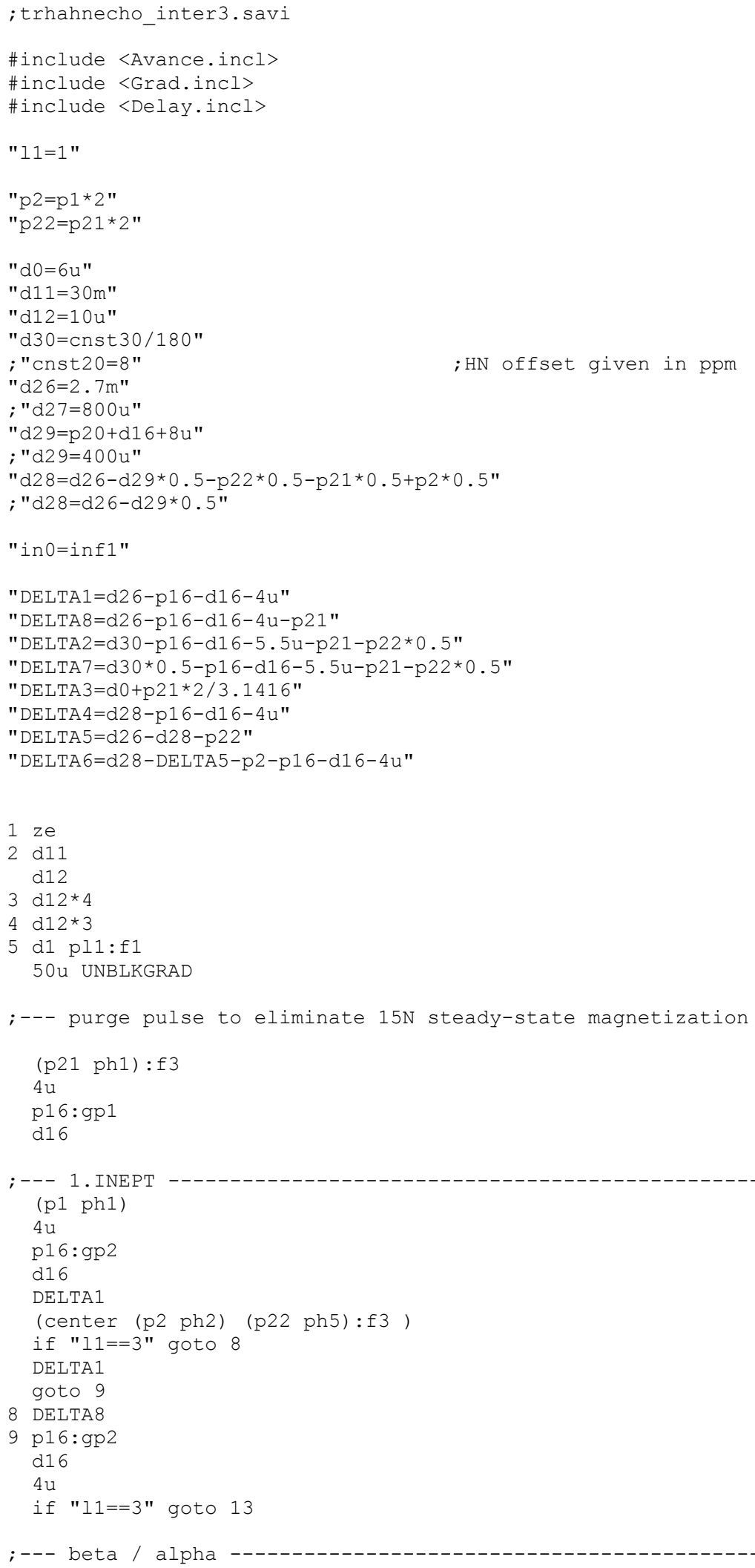




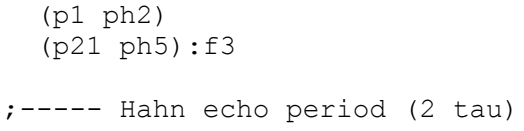




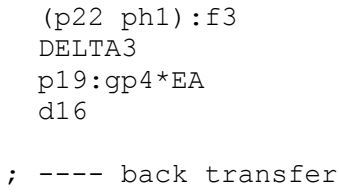

exit

ph $1=0$

ph $2=$

ph $3=2$

ph $4=3$

ph $5=0 \quad 2$

ph7=0 022

ph $8=1$

ph $9=0$

$p h 31=2 \quad 0 \quad 0 \quad 2$

pl1 : fl channel - power level for pulse (default)

;pl3 : f3 channel - power level for pulse (default)

;pl : fl channel - 90 degree high power pulse 
ip2 : f1 channel - 180 degree high power pulse ;p16: homospoil/gradient pulse

;p19: gradient pulse 2

;p20: gradient pulse 3

ip21: f3 channel - 90 degree high power pulse

;p22: f3 channel - 180 degree high power pulse

; d0 : incremented delay (2D)

;d1 : relaxation delay; 1-5 * $\mathrm{T} 1$

;d11: delay for disk I/O

; d16: delay for homospoil/gradient recovery

; $\mathrm{d} 26: 1 /(4 \mathrm{~J}) \mathrm{YH}$

; cnst $4:=\mathrm{J}(\mathrm{YH})$

ino: $1 /(2 * \mathrm{SW}(\mathrm{X}))=\mathrm{DW}(\mathrm{X})$

; ndo: 1

iNS: $4 * n$

; DS: 16

; td1: number of experiments

; FnMODE: echo-antiecho

; cnst20: HN center in ppm

; cnst30: integer $\mathrm{N}$ defining Hahn echo delay
$[1 \mathrm{msec}]$

[250 usec]

[250 usec]

[ 6 usec]

[30 $\mathrm{msec}]$
; use gradient ratio:

; for z-only gradients:

; gpz1: $50 \%$

igpz2: $27 \%$

; gpz3: 47\%

; $9 p z 4: 80 \%$

igpz5: $42 \%$

igpz6: 35

;gpz $7: 16.2 \%$

- use gradient files:

igpnam1: SINE.100

; gpnam2: SINE.100

; gpnam3: SINE.100

igpnam4: SINE.32

; gpnam5: SINE.100

;gpnam6: SINE.100

; gpnam7: SINE.32

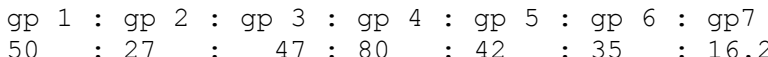




\section{F.4 Acquisition parameters for chemical exchange rates measured on wt}

\section{hVDAC1}

\#\#TITLE = Parameter file, TOPSPIN

Version 2.1

\#\# JCAMPDX $=5.0$

\#\#DATATYPE $=$ Parameter Values

\#\#NPOINTS $=51$ \$ modification sequence number

\#\#ORIGIN= Bruker Biospin GmbH

\#\#OWNER $=$ nmr900

\$ 2010-03-29 09:10:02.711 +0200 nmr900@nmr900

\$ /opt/topspin/data/savi/nmr/hvdac100325 Rex/18/acqus

\$\$ process /opt/topspin/prog/mod/shimcntl

$\# \#$ \#ACQTO $=1000000$

$\# \# \$ A M P=(0 \ldots 31)$

$100100100100100100100 \quad 100 \quad 100 \quad 100 \quad 100 \quad 100 \quad 100 \quad 100100 \quad 100 \quad 100 \quad 100$

100100100100100100100100100100100100100100

\#\# \$ANAVPT $=-1$

$\# \#$ \# $\mathrm{AQSEQ}=0$

$\# \#$ \#AQ $\bmod =3$

$\# \# \$ A \cup \bar{N} M=<$ au zgonly $>$

\#\# \$AUTOPOS $=\overline{<>}$

$\# \#$ \# $\$$ B $1=900.1$

$\# \# \mathrm{BF} 2=900.1$

\#\# \$BF3=91.206341

$\# \# \mathrm{BF} 4=900.1$

$\# \#$ \# $\$$ F $5=900.1$

$\# \#$ \#F $6=900.1$

$\# \#$ \$BF7 $=899.9$

$\# \#$ \# $\$$ F $8=899.9$

$\# \#$ \#YTORDA $=1$

$\# \#$ \# $\mathrm{CFDGTY}=2$

$\# \#$ \# $\mathrm{CFRGTYP}=5$

\#\# \$CHEMSTR $=\langle$ none $>$

\#\# $\$ \mathrm{CNST}=(0.63)$

$\begin{array}{lllllllllllllllllllllllllllllll}1 & 1 & 140 & 1 & 90 & 1 & 1 & 1 & 1 & 1 & 1 & 1 & 1 & 1 & 1 & 1 & 1 & 1 & 4.755 & 8.5 & 8.4 & 176 & 54 & 39 & 120 & 1 & 70\end{array}$

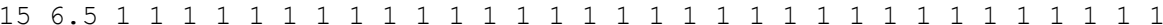

111

\#\# $\$$ CPDPRG $=<>$

\#\# \$CPDPRG1 $=\langle$ mlev $\rangle$

$\# \#$ \# CPDPRG2 $=\langle\mathrm{mlev}\rangle$

\#\# \$CPDPRG3 $=\langle$ mlev $>$

\#\# \$CPDPRG4 $=\langle\mathrm{mlev}\rangle$

\#\# $\$$ CPDPRG $5=\langle$ mlev $>$

\#\# \$CPDPRG $6=\langle\mathrm{mlev}\rangle$

\#\# \$CPDPRG7 $=\langle\mathrm{mlev}\rangle$

\#\# $\$$ CPDPRG $8=\langle\mathrm{mlev}\rangle$

\#\# $\$$ CPDPRGB $=\langle>$

\#\# \$CPDPRGT $=<>$

$\# \# \mathrm{D}=(0 \ldots 63)$

$6 e-0610.00360000000 .12000 .03 \quad 1 e-05 \quad 5 e-060000.00010005 e-05$

$\begin{array}{lllllllllllllllllllllll}0 & 0 & 0 & 0 & 0 & 0 & 0.0027 & 0 & 0.002461 & 0.000358 & 0.005434783 & 0 & 0 & 0 & 0 & 0 & 0 & 0 & 0 & 0 & 0\end{array}$

$0 \begin{array}{llllllllllllllllllllll}0 & 0 & 0 & 0 & 0 & 0 & 0 & 0 & 0 & 0 & 0 & 0 & 0 & 0 & 0 & 0 & 0 & 0 & 0 & 0 & 0 & 0\end{array}$

$\# \#$ \#ATE $=1269651137$

$\# \# \$ D B L=(0 \ldots 7)$

$\begin{array}{llllllll}120 & 120 & 120 & 120 & 120 & 120 & 120 & 120\end{array}$

$\# \# \mathrm{DBP}=(0.7)$

$150 \quad 150 \quad 150 \quad 150 \quad 150 \quad 150 \quad 150 \quad 150$

$\# \#$ \#BP0 $7=0$

\#\# \$DBPNAM0 $=<>$

\#\# \$DBPAM1 $=<>$

$\# \#$ \# DBPNAM2 $=<>$

\#\# \$DBPNAM3 $=<>$

\#\# \$DBPNAM4 $=<>$

\#\# \$DBPNAM5 $=<>$

\#\# \$DBPAM $6=<>$

\#\# \$DBPNAM7 $=<>$

$\# \# \$ \mathrm{DBPOAL}=(0 \ldots 7)$

$\begin{array}{llllllll}0.5 & 0.5 & 0.5 & 0.5 & 0.5 & 0.5 & 0.5 & 0.5\end{array}$

\#\# \$DBPOFFS $=(0 \ldots 7)$

$\begin{array}{lllllllll}0 & 0 & 0 & 0 & 0 & 0 & 0 & 0\end{array}$ 
$\# \# \$ D E=6.5$

\#\#\$DECBNUC= <off>

\#\#\$DECIM= 12

$\# \# \$ D E C N U C=\langle$ off $>$

$\# \#$ \#DECSTAT $=4$

\#\#\$DIGMOD $=1$

$\# \#$ DIGTYP $=8$

$\# \# \$ D L=(0 \ldots 7)$

$\begin{array}{llllllll}10 & 120 & 120 & 120 & 120 & 120 & 120 & 120\end{array}$

$\# \# \$ D P=(0 \ldots 7)$

$\begin{array}{lllllllll}150 & 150 & 150 & 150 & 150 & 150 & 150 & 150\end{array}$

$\# \#$ \#P $07=0$

\#\#\$DPNAME $0=<>$

\#\# \$DPNAME $1=<>$

\#\# \$DPNAME $2=<>$

\#\#\$DPNAME $3=<>$

\#\# \$DPNAME $4=<>$

\#\#\$DPNAME $5=<>$

\#\#\$DPNAME $6=<>$

\#\#\$DPNAME $7=<>$

$\# \#$ DPOAL $=(0 . .7)$

$\begin{array}{llllllll}0.5 & 0.5 & 0.5 & 0.5 & 0.5 & 0.5 & 0.5 & 0.5\end{array}$

$\# \#$ DPOFFS $=(0 \ldots 7)$

$\begin{array}{lllllllll}0 & 0 & 0 & 0 & 0 & 0 & 0 & 0\end{array}$

$\# \#$ \#QDMODE $=0$

$\# \# \$ \mathrm{DR}=17$

\#\#\$DS $=16$

\#\# \$DSLIST $=<$ SSSSSSSSSSSSSS $>$

\#\#\$DSPFIRM $=0$

\#\#\$DSPFVS $=12$

$\# \#$ \#TYPA $=0$

$\# \#$ \#XP $=<>$

\#\#\$F1LIST $=\langle 111111111111111>$

\#\#\$F2LIST $=\langle 222222222222222>$

\#\#\$F3LIST $=\langle 333333333333333\rangle$

$\# \#$ \#CUCHAN $=(0 \ldots 9)$

$\begin{array}{lllllllllll}0 & 2 & 0 & 3 & 0 & 0 & 0 & 0 & 0 & 0\end{array}$

$\# \# \mathrm{EL} 1=90$

$\# \#$ \#L2 $=90$

$\# \#$ \#L $3=90$

$\# \#$ \#LL4 $=90$

$\# \# \$ F O V=20$

\#\#\$FQ1LIST $=\langle$ freqlist $>$

$\# \# \$ F Q 2$ LIST $=\langle$ caco.savi $>$

$\# \#$ \$FQ3LIST $=\langle$ freqlist $>$

\#\#\$FQ4LIST $=\langle$ freqlist $>$

$\# \#$ \#Q5LIST $=\langle$ freqlist $>$

\#\#\$FQ6LIST $=<$ freqlist $>$

$\# \#$ \#FQ7LIST $=\langle$ freqlist $>$

\#\#\$FQ8LIST $=\langle$ freqlist $>$

\#\#\$FRQLO3= 1899271.84466019

$\# \#$ \#RQLO3N=0

\#\#\$FS $=(0 \ldots 7)$

$\begin{array}{llllllll}83 & 83 & 83 & 83 & 83 & 83 & 83 & 83\end{array}$

$\# \#$ \# $\mathrm{FTLPGN}=0$

$\# \# \mathrm{FW}=125000$

$\# \#$ \#nMODE $=0$

$\# \#$ \# $\mathrm{ETYPE}=0$

\#\#\$GP031=0

\#\# \$GPNAM0 $=\langle$ sine. 100$\rangle$

\#\#\$GPNAM1 $=\langle$ SINE.100 $\rangle$

\#\# \$GPNAM1 $0=\langle$ sine. 100$\rangle$

\#\#\$GPNAM1 $1=\langle$ sine. 100$\rangle$

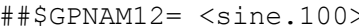

\#\# \$GPNAM1 $3=\langle$ sine. 100$\rangle$

\#\#\$GPNAM1 $4=\langle$ sine. 100$\rangle$

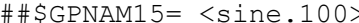

\#\#\$GPNAM1 $6=\langle$ sine.100 $\rangle$

\#\#\$GPNAM1 $7=\langle$ sine. 100$\rangle$

\#\#\$GPNAM1 $8=\langle$ sine. 100$\rangle$

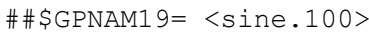

\#\# \$GPNAM $2=\langle$ SINE. 100$\rangle$

\#\# \$GPNAM20 0 <sine. 100$\rangle$

\#\# \$GPNAM21 $=\langle$ sine. 100$\rangle$ 


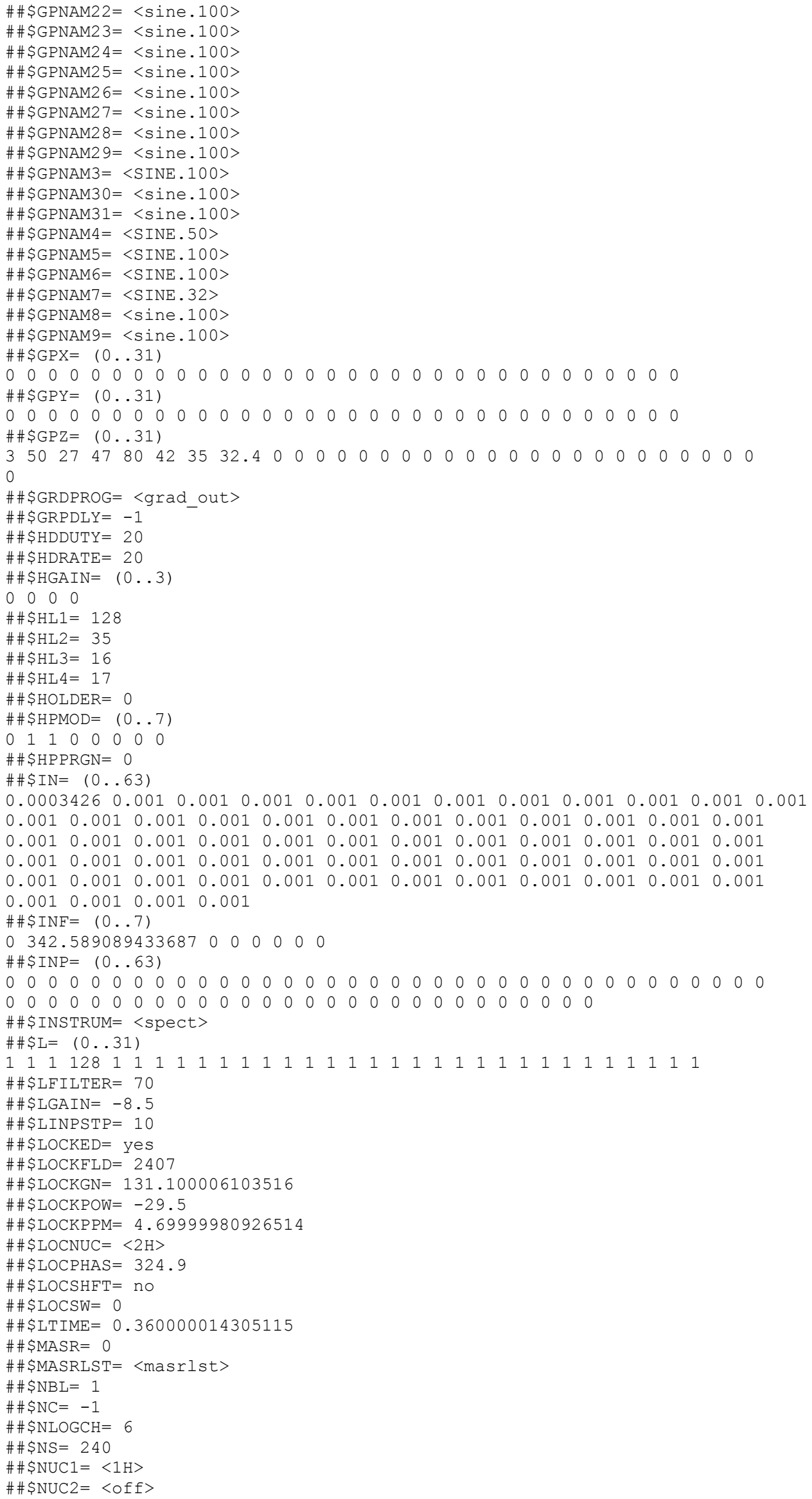




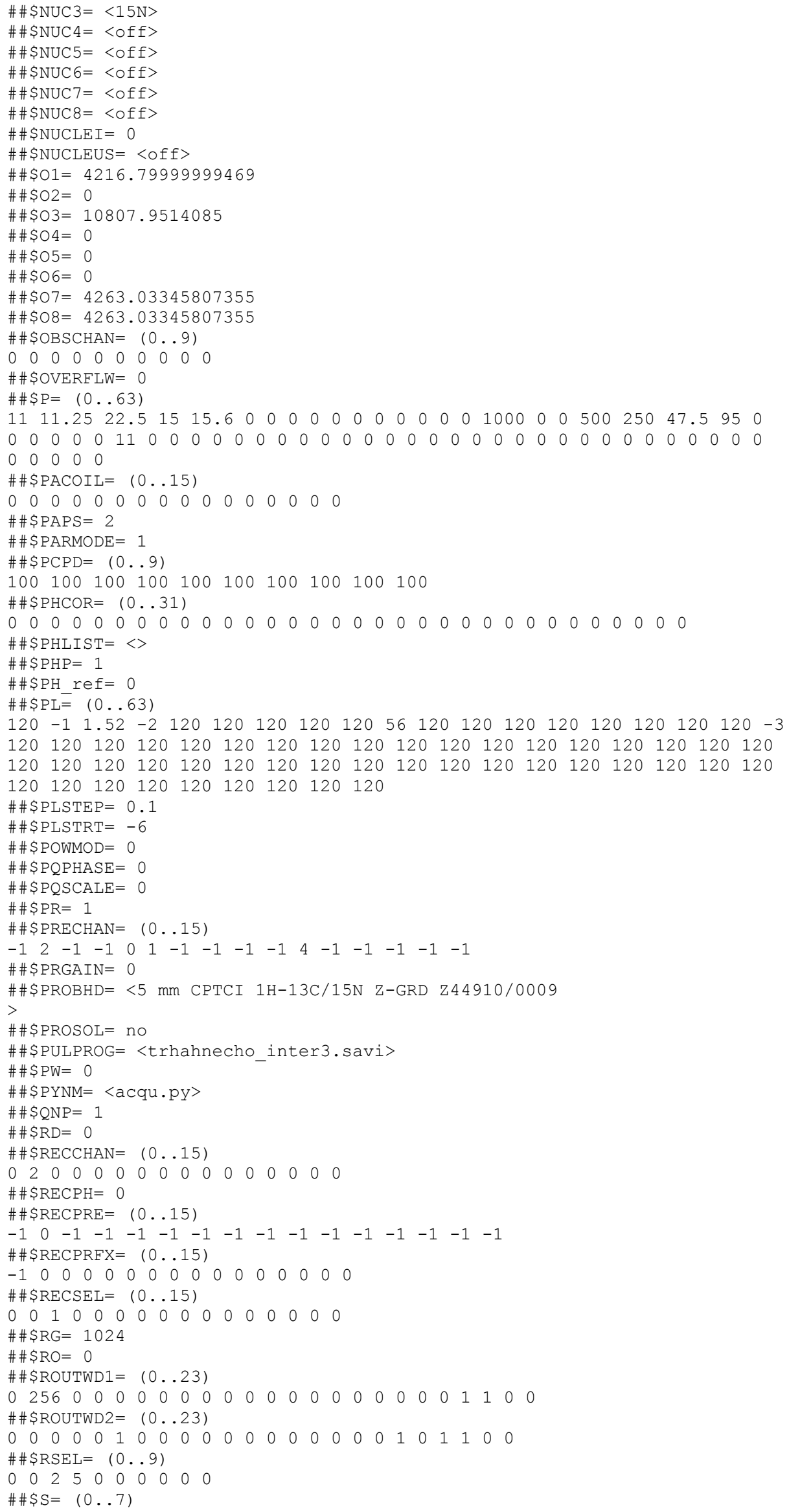




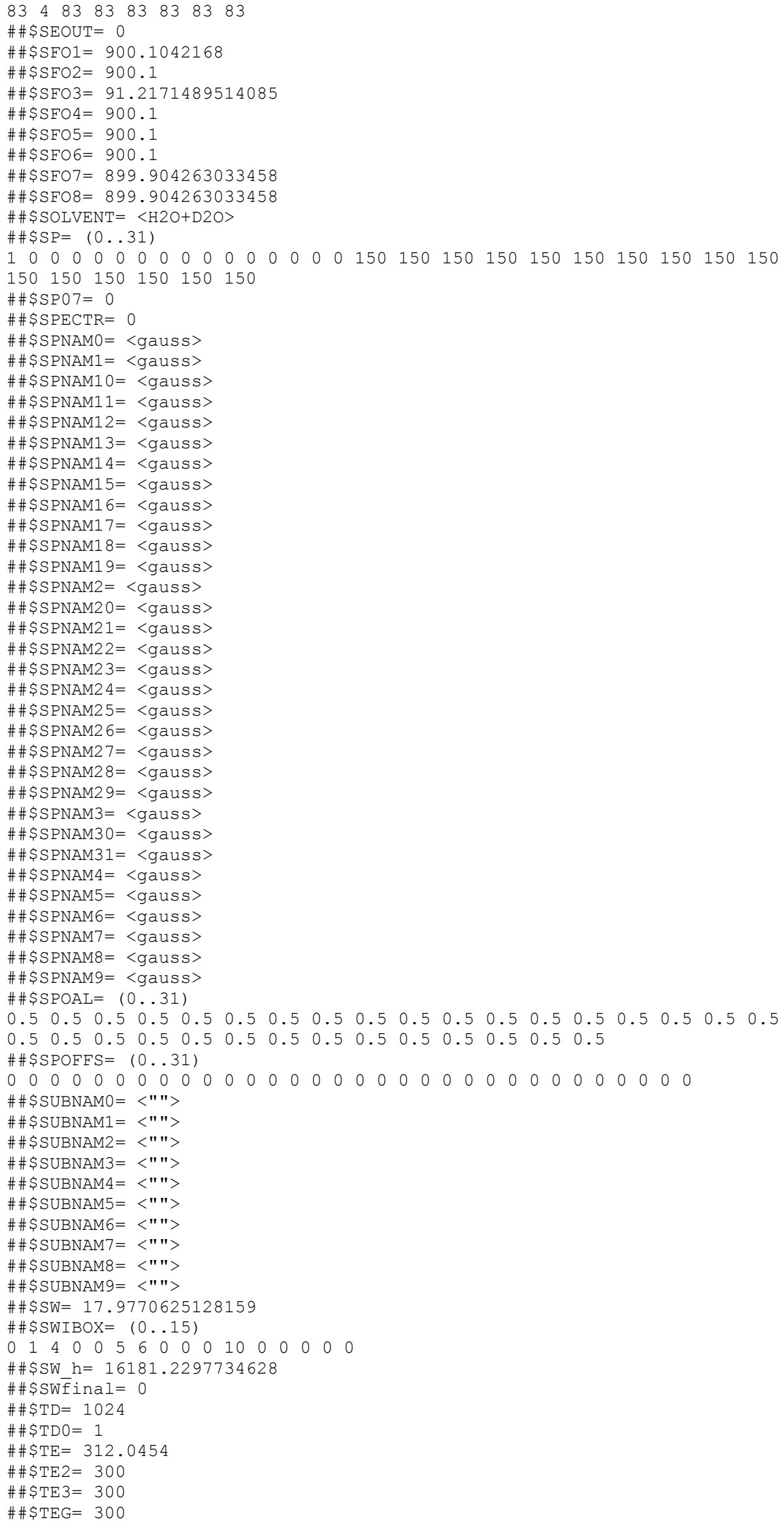




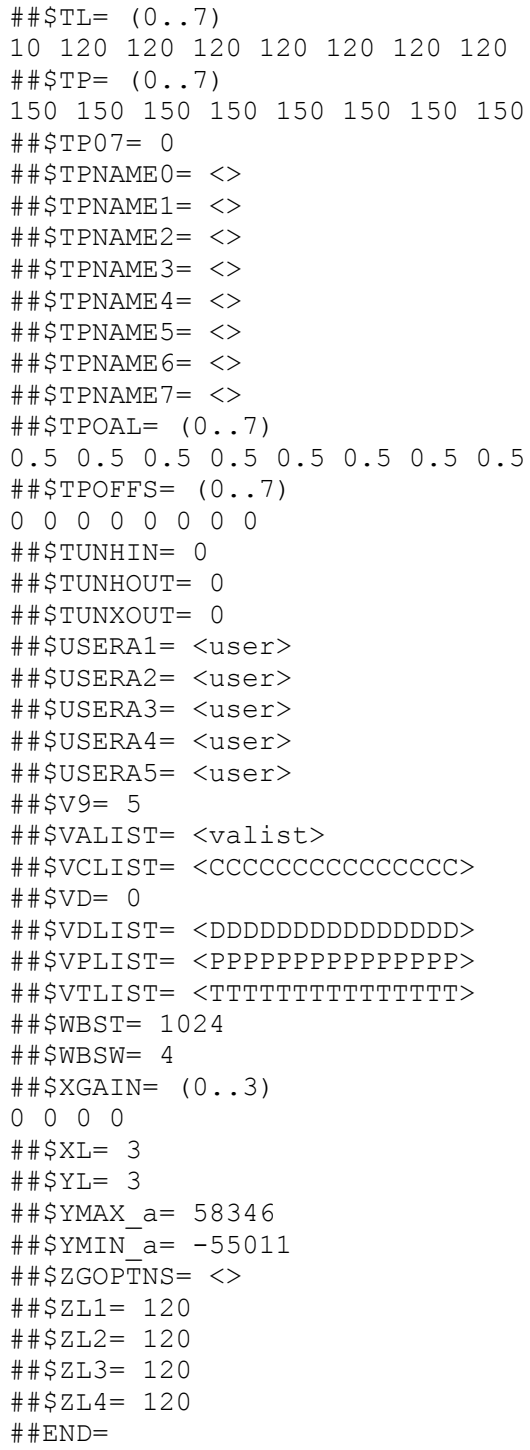

